\title{
Effects of arginine derivatives and oligopeptides on the physical properties of model membranes
}

\author{
Dissertation \\ for the award of the degree \\ "Doctor rerum naturalium" \\ of the Georg-August-Universität Göttingen
}

within the doctoral program Physics of Biological and Complex Systems

of the Georg-August University School of Science (GAUSS)

submitted by

Sarah Félice Verbeek

from The Hague, Netherlands

Göttingen 2020 


\section{Thesis committee}

Prof. Dr. Andreas Janshoff

Institute of Physical Chemistry

Georg-August-University of Göttingen

Prof. Dr. Silvio Rizzoli

Department of Neuro- and Sensory Physiology

University Medical Center Göttingen

Prof. Dr. Reinhard Jahn

Department of Neurobiology

Max Planck Institute for Biophysical Chemistry

\section{Examination Board}

Referee: Prof. Dr. Andreas Janshoff

Institute of Physical Chemistry

Georg-August-University of Göttingen

$2^{\text {nd }}$ Referee: Prof. Dr. Silvio Rizzoli

Department of Neuro- and Sensory Physiology

University Medical Center Göttingen

Prof. Dr. Reinhard Jahn

Department of Neurobiology

Max Planck Institute for Biophysical Chemistry

Dr. Sarah Adio

Institute for Microbiology and Genetics

Georg-August-University of Göttingen

Prof. Dr. Marcus Müller

Institute for Theoretical Physics

Georg-August-University of Göttingen

Prof. Dr. Kai Tittman

Department of Molecular Enzymology

Georg-August-University of Göttingen

Date of examination: 10.03 .2020 


\section{Declaration}

I, Sarah Verbeek, hereby certify that my doctoral thesis entitled "Effects of arginine derivatives and oligopeptides on the physical properties of model membranes," has been written independently and with no other sources and aids than quoted.

Göttingen, 29.01.2020

Sarah Verbeek 

To my family, my friends and my bunny.

"But not to have music would be unnatural to me."

Floor Jansen, lead singer of $\mathcal{N i g h t w i s h ~ 2 0 1 3 - p r e s e n t ~}$ 



\section{ABSTRACT}

Cell-penetrating peptides (CPPs) are often rich in arginine. They are able to cross cell membranes and carry other molecules into cells without being cytotoxic. The mechanisms by which they accomplish this are still controversial, although endocytotic pathways or spontaneous entry mechanisms have both been proposed. This work takes a bottom-up approach to the role of arginine in CPPs, by comparing an arginine side chain mimic with arginine oligopeptides of different length, with respect to their influence on membrane model systems of various lipid compositions. Simulations as well as breakthrough force spectroscopy experiments showed that the arginine sidechain mimic increased likelihood of pore formation in negatively charged membranes. This destabilisation of membranes was not found for hexa-arginine, a known CPP, for any lipid composition or peptide concentration, neither in breakthrough force spectroscopy nor by liposome leakage assays. However, hexa-arginine was shown to induce membrane stack formation on negatively charged solid-supported lipid bilayers at low peptide concentration. It was also found that arginine peptides of any length, as well as hexa-lysine, were able to cause stack formation in these membrane compositions, provided that peptide concentration was high enough. Our findings combined with literature mention of lamellarisation of model membranes and cell membranes caused by arginine-rich peptides suggest that membrane stack formation and lamellarisation is likely an important aspect of oligoarginine CPPs' mode of action. However, our data do not support membrane disruption or pore formation as a means of cell entry. Future work should determine whether our findings are reproducible for longer oligoarginines, and provide final proof to reject the hypothesis of pore formation. Overall, this work points to a non-pore forming mode of action of CPPs, at least for shorter oligoarginines. 



\section{CONTENTS}

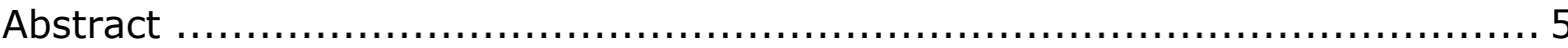

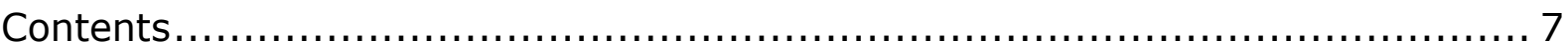

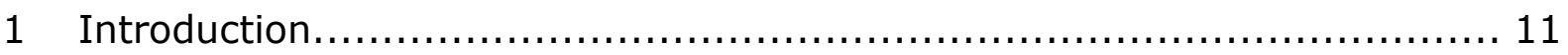

$1.1 \quad$ Historical background \& classification of CPPs ....................... 12

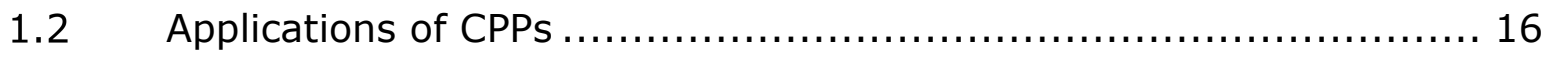

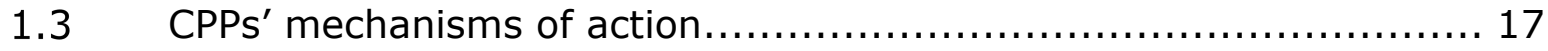

1.3.1 Excursus: parallels between CPPs and AMPs ........................ 19

1.3.2 Cell-entry mechanisms for arginine-rich CPPs ...................... 21

$1.4 \quad$ Scope of the thesis............................................................ 24

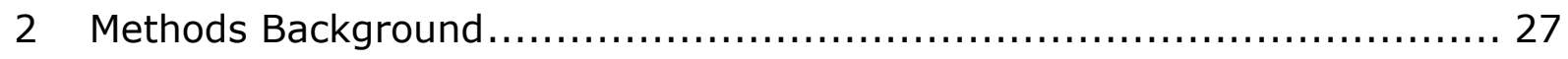

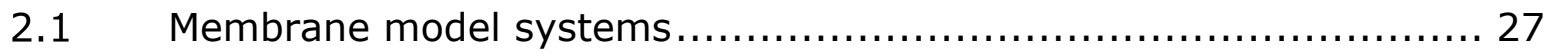

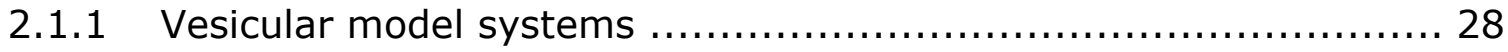

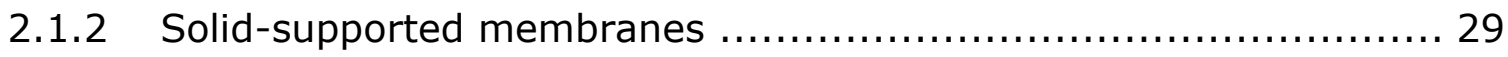

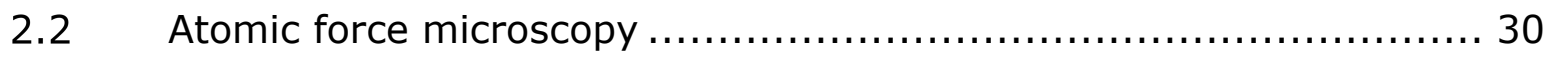

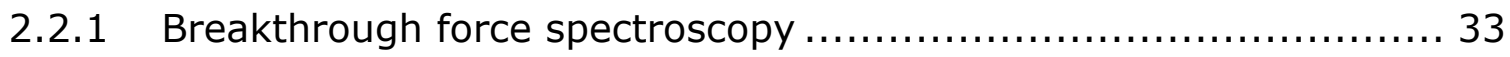

2.2.2 Theoretical background: contact regime .............................. 34

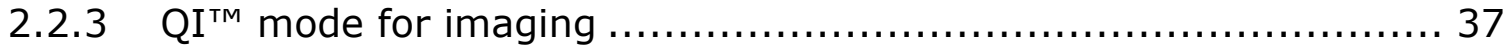

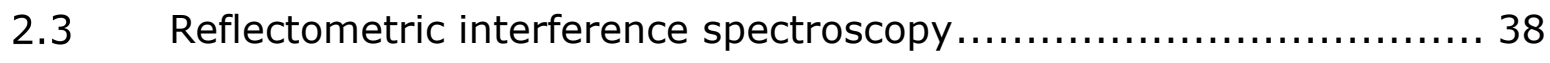

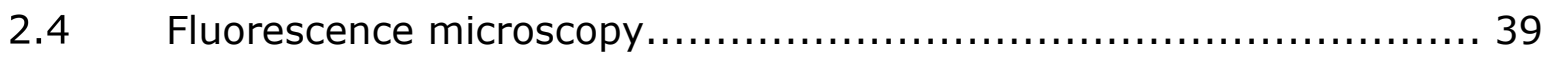

2.5 Simulations of pore formation ......................................... 41

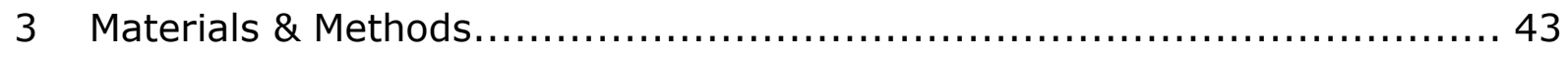

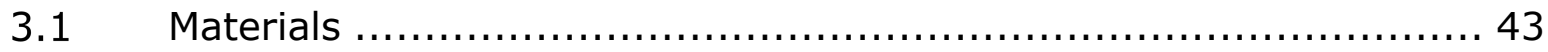




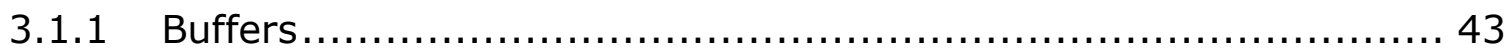

3.1 .2 Lipids....................................................................... 44

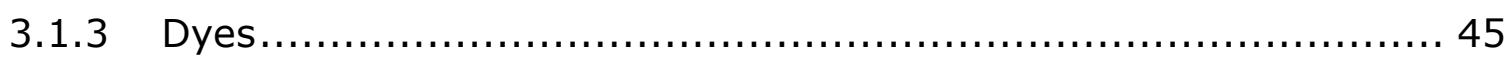

3.1.4 Arginine derivatives and peptides.................................... 46

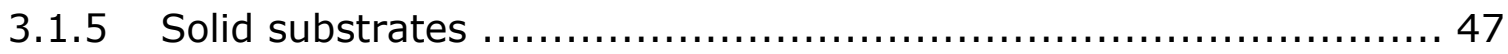

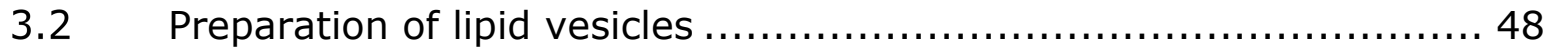

3.2.1 Preparation of lipid films in desired compositions.................... 48

3.2.2 Preparation of vesicles ................................................ 49

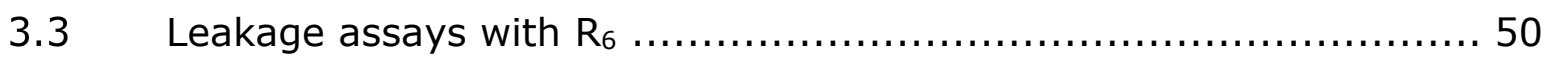

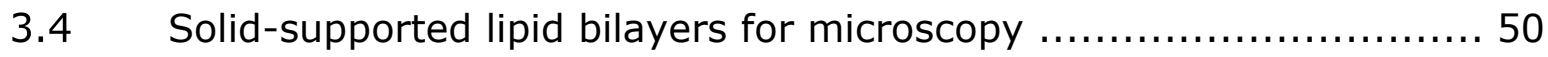

3.4.1 Silicondioxide-supported membranes ................................ 50

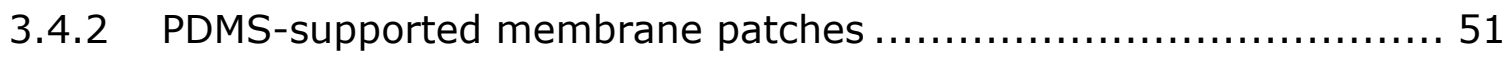

3.4.3 Addition of R-derivatives............................................. 51

3.4.4 Addition of vesicles ................................................. 52

3.4.5 In situ staining of membrane stacks with Rh18 $\ldots \ldots \ldots \ldots \ldots \ldots \ldots \ldots \ldots \ldots \ldots \ldots \ldots \ldots$

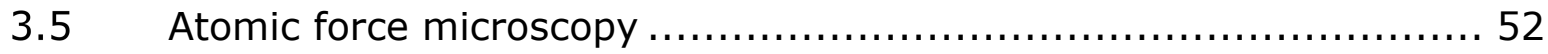

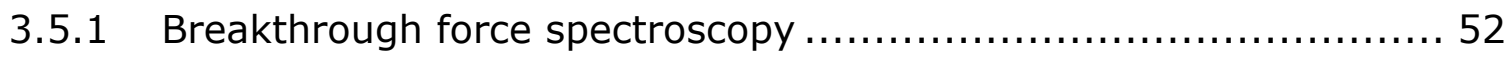

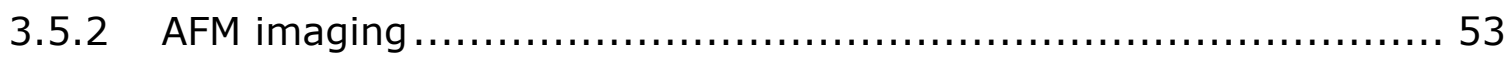

3.5.3 Data processing …..................................................... 53

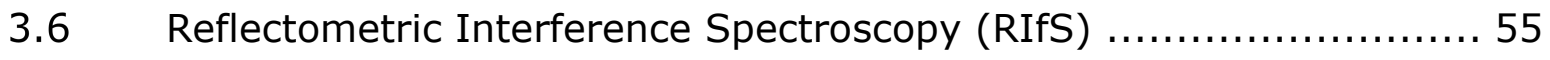

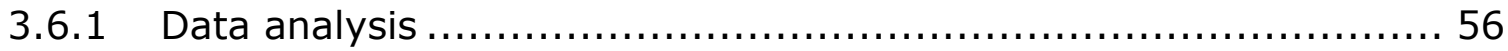

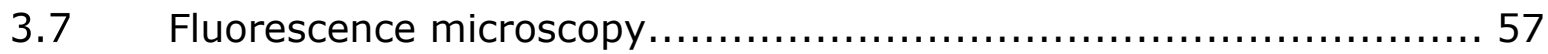

3.7.1 Fluorescence monitoring after R-derivative addition................. 58

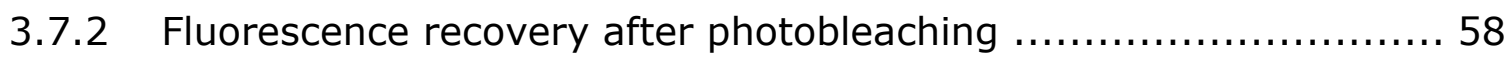

4 Effects of arginine derivatives on different lipids..............................6 61

4.1 Simulations of pore formation ......................................... 62

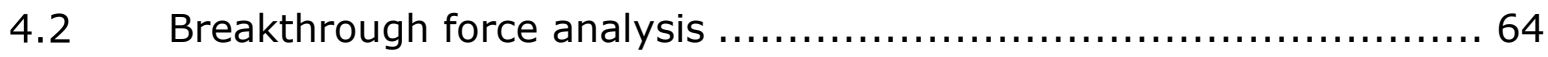

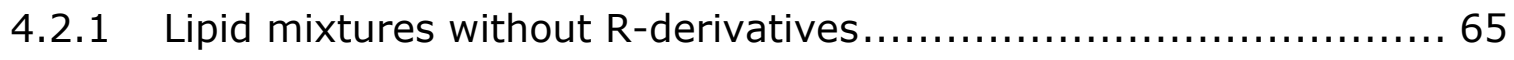


4.2.2 Breakthrough force results with R-derivatives ....................67 67

4.2.3 Breakthrough distance results with R-derivatives ................ 74

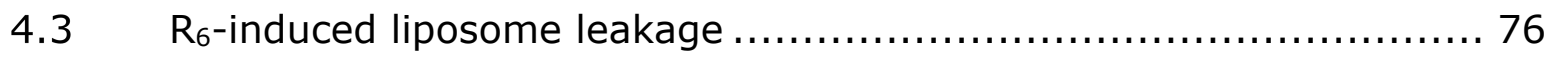

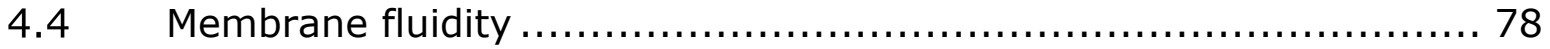

4.5 Visual effects of R-derivatives ...................................... 79

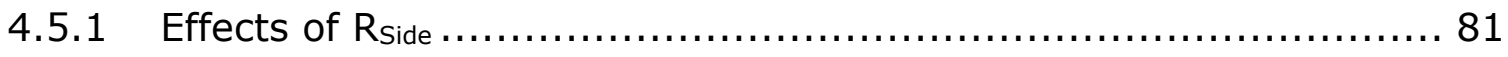

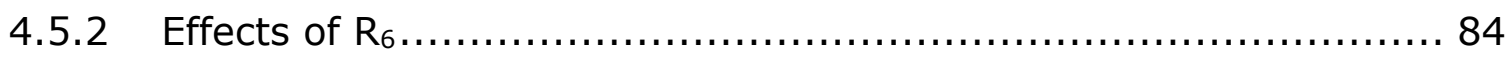

4.6 Discussion physical effects of R-derivatives...................... 86

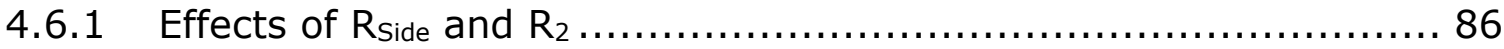

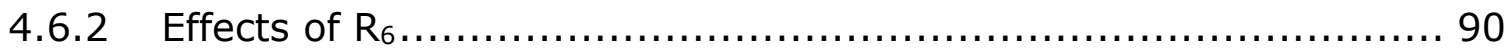

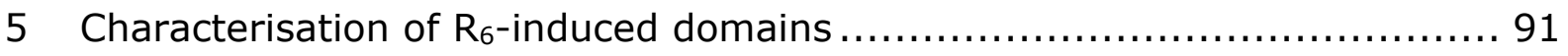

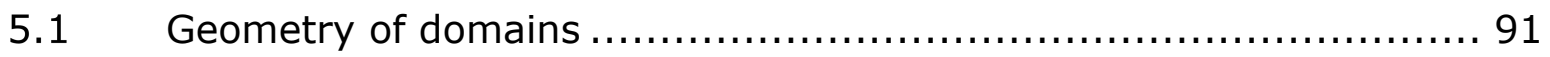

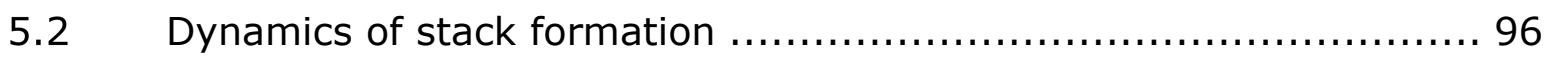

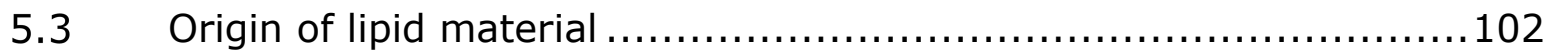

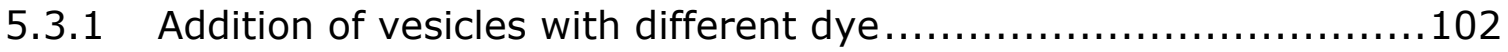

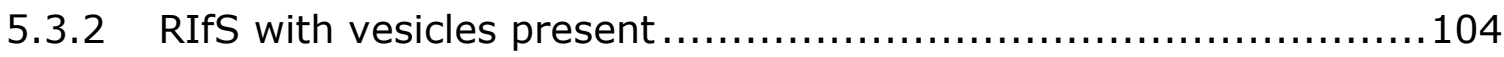

5.3.3 Membrane patches on PDMS ................................... 105

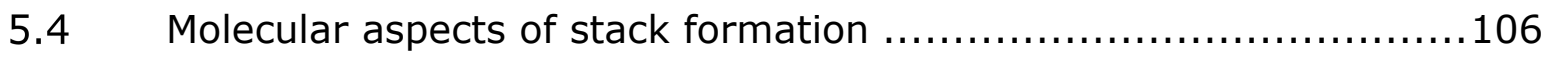

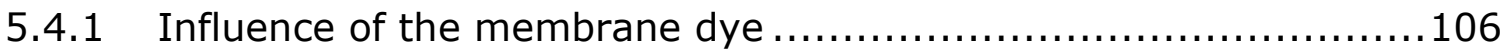

5.4.2 Peptides and lipids necessary for stack formation .................. 112

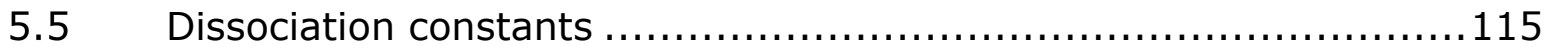

5.6 Discussion R-peptide induced stack formation .................... 120

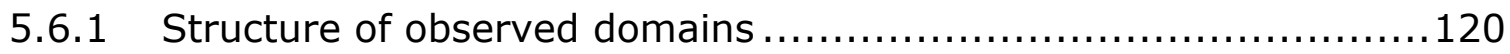

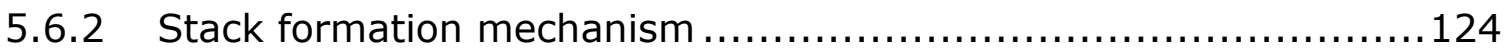

5.6.3 Molecular requirements for stack formation $\ldots \ldots \ldots \ldots \ldots \ldots \ldots \ldots \ldots \ldots \ldots \ldots \ldots$

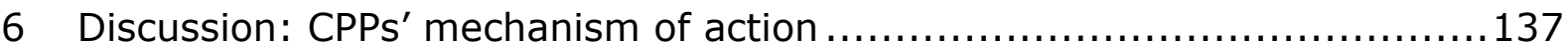

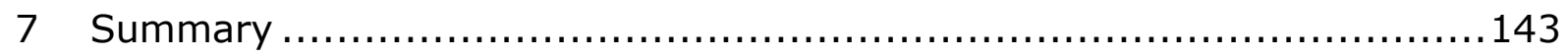

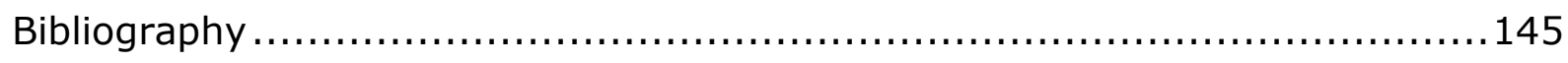


Appendix

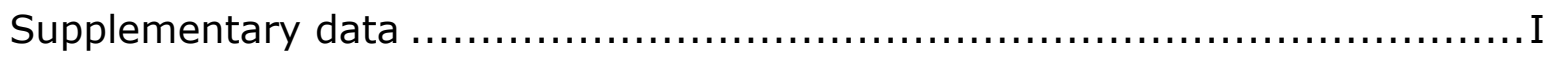

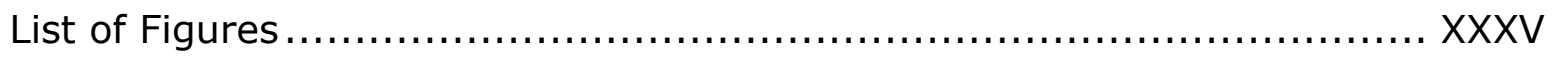

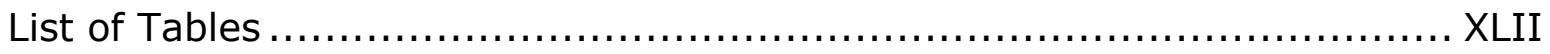

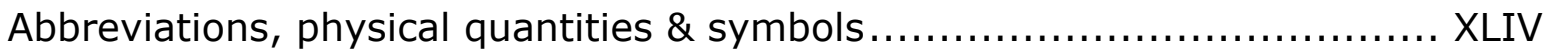

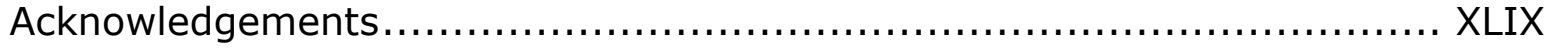




\section{INTRODUCTION}

Cell-penetrating peptides (CPPs) are widely applied in development of novel drug delivery strategies[1,2], due to their ability to transpose the cell membrane without causing apparent damage. Multiple mechanisms by which CPPs accomplish this have been proposed, but accurate mechanism predictions based on the sequence of peptides or the desired application cannot be made yet $[1,3-7]$. Many CPPs are rich in positively charged amino acid residues, especially arginine and lysine (Figure 1.1) and by far most have a net positive charge. Consensus has

A

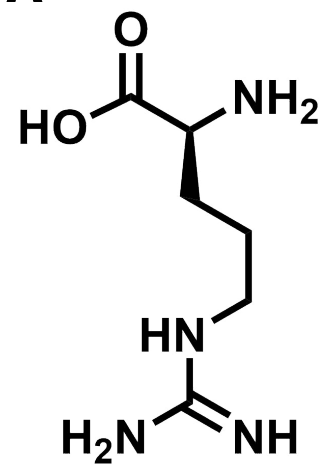

B

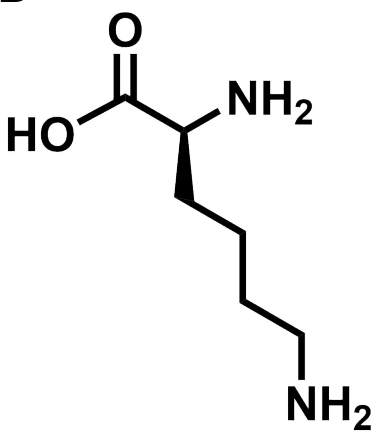

Figure 1.1: Basic amino acids L-arginine (A) and L-lysine (B) in neutral form.

been reached that interaction of this positive charge with negatively charged components in the cell membrane plays an important role in the mechanism of entry[5, 7], although positive charge alone is not enough[8,9]. In this work, we intend to shed more light on mechanisms of entry by a bottom-up approach: we will explore intrinsic effects arginine derivatives and oligopeptides have on different membrane compositions, by means of in vitro experiments and pore forming simulations.

First, the historical background of CPPs, their classification and their applications will be introduced, including examples of recent studies. Then, generally proposed mechanisms of entry for CPPs will be discussed, as well as two more recently 
proposed direct entry mechanisms for arginine-rich CPPs[10,11]. Lastly, the scope of this work will be sketched.

\subsection{Historical background \& classification of CPPs}

The vast realm of CPP research was born in the late 1980 s and early 1990s. In 1988 , it was discovered that the trans-activator protein for transcription (TAT) of the HIV-1 virus can transpose the plasma membrane on its own $[12,13]$. Later, it was discovered that shorter segments of this 82-amino acid long protein were sufficient to accomplish translocation: TAT ${ }_{48-60}$ GRKKRRQRRRPPQ[14] and TAT $_{49-57}$ RKKRRQRRR[15]. Briefly after discovery of TAT translocation, in 1991, it was found that the 60-amino acid homeodomain of antennapedia gene homeobox of Drosophila Melanogaster (denoted pAntp) translocated into nerve cells[16]. Also for this peptide, a truncated version was later discovered to be sufficient for translocation[17]: RQIKIWFQNRRMKWKK, now known as penetratin. All these firstly discovered CPPs are rich in arginine $(R)$ and another basic amino acid, lysine (K). In 2000, it was shown that also peptides entirely consisting of arginine could translocate into cells $[8,18]$, with 9 residues $\left(R_{9}\right)$ being the optimal length[8] and arginine peptides being generally more potent than peptides consisting of other positively charged amino acids (lysine or ornithine)[18]. It has to be noted however, that literature has mentioned enhancement of delivery of markers into tumour cells by cationic homopolymers long before CPPs were discovered and named as $\operatorname{such}[19,20]$.

Since the discovery of TAT and pAntp derivatives, many more cell-penetrating peptides, modified peptides and peptide derivatives have been discovered and reviewed along with their applications numerous times[1, 3, 4, 6, 21-28]. Efforts have been made to keep an overview of all CPPs by Gautam et al. with the creation of the CPPSite platform[29]. This interactive website can be queried by sequence and literature to that sequence can be found, as well as all data available and calculated physicochemical properties. At the time of creation in 2012 it contained 874 sequences. After a revision in 2015, this had more than doubled to 1850 sequences[30]. 
CPPs are mostly classified according to the amino acids in their sequence, rather than by mode of action or general use: there is not one clear mechanism or function for one peptide[4], as both depend on experimental conditions[31] and even what cargo is attached to the peptide[32] (with the purpose of drug delivery). According to amino acid composition, CPPs can be classified as cationic, amphipathic and hydrophobic CPPs.

Cationic CPPs mostly consist of positively charged amino acids, such as lysine $(K)$, arginine $(\mathrm{R})$, histidine $(\mathrm{H})$ or a shorter version of lysine, ornithine. Many peptides in this category are either TAT-derived or oligoarginines[1, 24]. It was first suggested that at least 8 arginine residues were necessary for cell entry[8, 33], but literature shows contradicting results: $R_{6}$ [34] and even $R_{5}$ [35] are being applied in therapeutic application development as well.

Numerous studies have directly shown that R-peptides are more effective as a CPP than lysine, in model systems[36] as well as for delivery into cells[8, 18, 33, 37]. Mutations of lysine to arginine can enhance the cellular uptake of a peptide[38]. This is ascribed to the ability of the guanidine moiety in the arginine sidechain to form bidentate bonds to negatively charged components in the cell membrane[3]. Amphipathic CPPs contain both hydrophilic and hydrophobic amino acids (such as alanine $(A)$, leucine $(L)$, valine $(V)$ and isoleucine $(I))$. They are further divided into primary, secondary, and proline-rich amphipathic $\operatorname{CPPs}[1,24]$. Primary amphipathic CPPs are derived from naturally occurring proteins or peptides, or their sequence is a chimera of naturally occurring peptide sequences. Secondary amphipathic CPPs are denoted such, because their hydrophilic and hydrophobic amino acids are located on separate faces of a secondary structure, such as an a-helix. Proline-rich CPPs are, as their name betrays, rich in the amino acid proline. Very proline-rich peptides can form the characteristic helical secondary structure, denoted polyproline II[24].

Hydrophobic CPPs have no or very low net charge and are rich in amino acids with hydrophobic sidechains. It has been reported that the hydrophobic residues can be randomly scrambled, without impairing cellular uptake[24]. This class of CPPs has gained much less research interest than the other two classes[1].

Several attempts have been made to categorise CPPs according to their effectivity or physicochemical properties coupled to their mechanism of action[4, 7, 39], 
twice with the help of CPPSite, but still no definitive classification could be given. Nevertheless, some important takes from such comparative research are worth discussing.

The first is the repeated acknowledgement that experimental conditions to study CPP effectivity lack harmony. This makes statistical comparison and ranking of CPPs, as well as predicting their behaviour, very challenging $[4,7,39]$. Hence, only general important points of attention could be recommended, or rough statistical trends on physicochemical properties, could be given. Stalmans et al. performed rigorous statistical cluster analysis of 136 CPP-sequences found in literature[39]. They defined the cell-penetrating response (CP-response) parameter to compare different peptides, which was a quantity normalised for concentration of the peptide, and further normalised to penetratin activity as a positive control. Cargo was neglected. This analysis suggested the importance of peptide shape and structural complexity to CPP potency, besides the already well-known influence of positive charge and amphipathic nature.

Similar general guidelines were concluded by Kauffman et al.[4], after having completed an analysis with the help of CPPSite, promising "a taxonomy for CPPs": this 'taxonomy' should classify CPPs' mode of action by five criteria. Among these criteria was the potential to induce extensive plasma membrane lysis, which is an undesirable property for CPPs. Other criteria were whether their uptake is spontaneous or energy-dependent, and whether their uptake mechanism involves transient plasma membrane disruption or endosomal membrane disruption (generally proposed mechanisms for CPP uptake will be discussed in Section 1.3, p. $17 \mathrm{ff}$.). Apart from this theoretical proposal for future attempts at systematic applications of CPPs, Kauffman et al. also visualised some interesting trends by statistical analysis of physicochemical properties of 747 cell-penetrating peptides. They revealed that known CPPs had a median of 14 residues, and the majority was between 10 and 20 residues in length. They also revealed that the median charge of CPPs was +5 , with an additional peak at net zero charge and only a small fraction being anionic. Also, even though a subset of CPPs exploits this property, most CPPs only have low amphipathicity when folded into an a-helix or $\beta$-sheet structure [4].

Trends similar to the above were recently observed by Ramaker et al.[7] when they compared 474 CPP sequence motifs experimentally, after obtaining them from CPPSite. CPPSite had 741 unique sequence entries at the time of their query. 
They excluded peptides which contained non-natural amino acids or otherwise unnatural modifications, such as circular peptides, as well as peptides which were longer than 24 amino acid residues. They systematically assessed cellular uptake as well as toxicity of all 474 sequences by labelling them with carboxyfluorescein and performing systematic intensity analysis in cells. They found a strong correlation between uptake efficiency and positive charge, as well as sequence length (peptides longer than 13 amino acids were significantly better than shorter peptides). Additionally, Ramaker et al. formulated a top 20 of CPPs[7], according to their uptake efficiency and low toxicity score. Remarkably, no homopolymers of arginine made it into this top 20; only polylysine $\left(\mathrm{K}_{19}\right)$ was found here, as well as $\mathrm{CWK}_{18}$. Arginine peptides of length 5-11 were tested. The top 20 contained CPPs of at least length 16 and net charge of at least +4 . All peptides in the top 20 contained at least arginine or lysine residues, and by far most were amphipathic according to CPPSite.

It is important to note that while this systematic experimental analysis of so many peptides is very informative, it has been reported that CPP behaviour and even mechanism[40] depends on experimental conditions[4], such as cargo[32, 41], type of cells, or even small mutations[40] or modifications such as the N-terminal cap[31]. Hence, a different set of experimental conditions might have delivered a different ranking. Furthermore, the widespread fluorescence intensity analysis with carboxyfluorescein is deemed problematic, as fluorophore intensity can easily be falsified, wrongly interpreted or influenced by $\mathrm{pH}[4]$.

Different lengths of arginine peptides, and modifications to them, have been compared as well. We will discuss 3 examples of this. The first is the work of Wender et al., who first demonstrated that arginine peptides have a better CPP activity than TAT49-57[8]. After this discovery, they went on and further compared $R_{6-12}$ to $D$-amino acid versions of the peptides, which performed even better. This finding was further confirmed in later literature[33]. The authors proceeded to explore the effect of modifications further, by designing 'peptoids' with an oligoglycine backbone, and the guanidine-bearing arginine-like sidechain attached to the amide nitrogen in the peptide bond. With this peptoid design, they avoided influence of chirality. They demonstrated that these peptoids were even more potent than the $\mathrm{D}$-peptides, if the arginine-like sidechain was extended to more atoms. 
The trend of better uptake upon reversal of chirality was not confirmed in studies of circular oligoarginines by Traboulsi et al. [42]. They synthesised several macrocyclic oligoarginines, in L- and D-form, as well as peptides that were part circular and part linear. They discovered that a heptamer with four R-residues in the circular portion and three more attached in a linear fashion ("Cyc-R4-R3") was just as efficient in terms of cellular uptake, as linear $R_{9}$.

\subsection{Applications of CPPs}

CPPs are mostly used as agents for drug delivery, in which their potential to transpose cell membranes is exploited to facilitate cell entry of drug molecules that would otherwise be useless[1,43]. Other components than drugs, including DNAs, siRNAs for transfection purposes, or proteins, can also be carried by CPPs[1].

'Cargo' can be delivered by CPPs via covalent linkage, non-covalent linkage[1, 4, $24]$, or incorporation of the CPP into a more complicated delivery system. Covalent linkage is mostly achieved via peptide bonds (via cloning for larger protein cargoes), a thioester bond or a disulphide bond[1]. Non-covalent linkage is mostly achieved via charge-charge interaction with the CPP and negatively charged cargo, usually DNA (example: [44]). With weak proof of complexation, $R_{5}$ has been successfully used in the delivery of non-covalently attached cargo[35]. An example of a more complicated delivery system is attachment to liposomes via attachment of the CPP (in examples octa-arginine) to a fatty acid anchor, together with specific target ligands[2, 45]. The liposomes carry the drugs[45] or the intended transfection material[2], and can be further modified by a PEG coating.

Potential therapeutic and clinical applications for CPPs were most recently reviewed in 2017[1] and specifically for cancer treatment strategies in 2019[46]. Many applications involved TAT-derivatives as a CPP[1]. An example of oligoarginine therapeutic application beside the recent ones mentioned above[2, 45], is the use of hepta-arginine $\left(R_{7}\right)$ in the development of a cream for the treatment of psoriasis, by mediating topical delivery of Cyclosporin-A[47]. More recently, arginine-rich CPPs have been demonstrated to have neuroprotective abilities[48, 49]: not only are they neuroprotective in rodent stroke models, it recently has been shown that 
the polyarginine $\mathrm{R}_{18}$ also reduces brain injury and improves functional outcomes for a primate stroke model.

\subsection{CPPs' mechanisms of action}

CPPs have been proven to be taken up by multiple mechanisms, sometimes even multiple at the same time. Generally, CPP mechanisms of entry are divided in

\section{Energy-dependent mechanisms}

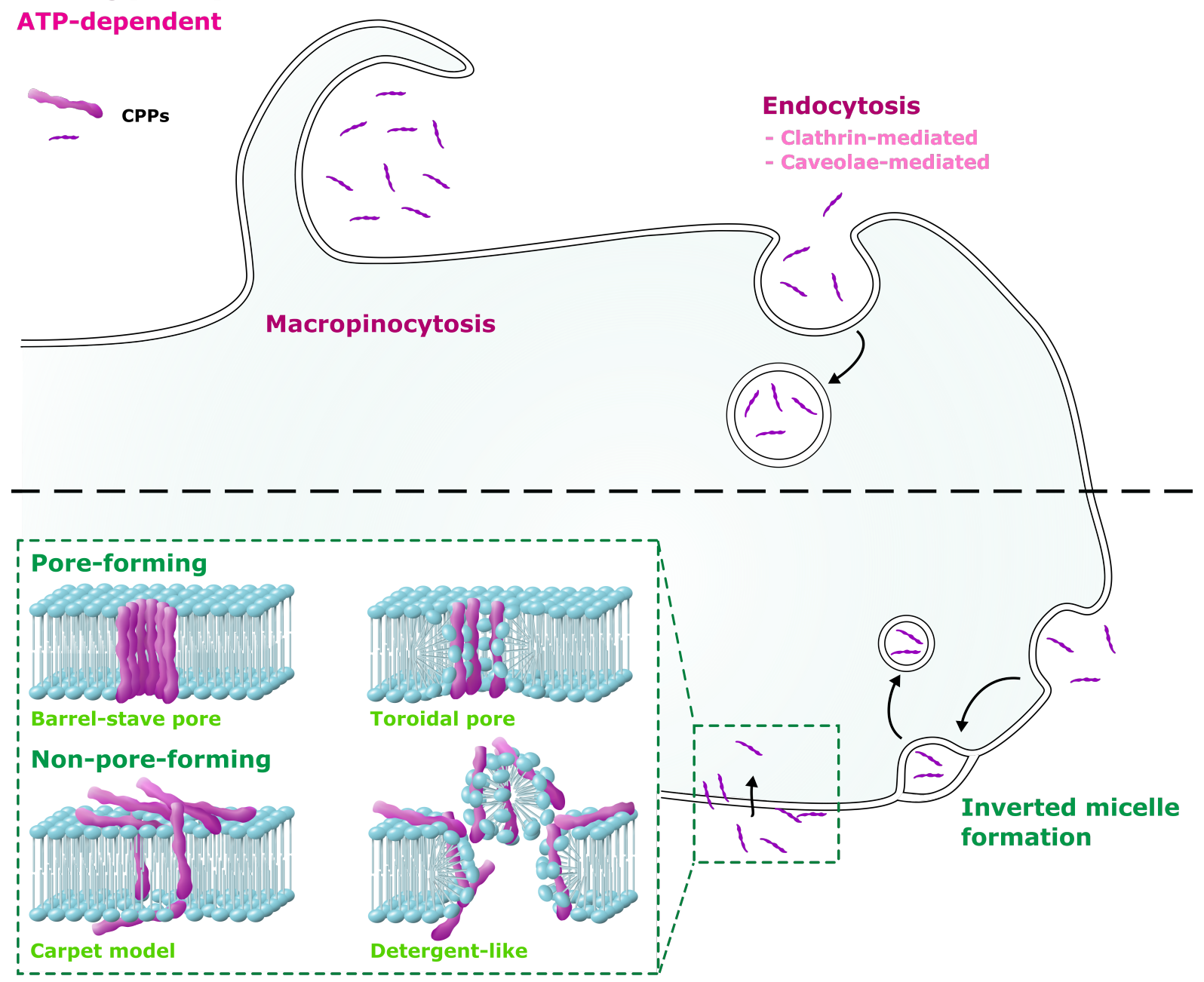

Energy-independent mechanisms

Spontaneous translocation

Figure 1.2: Generally proposed mechanisms of entry for CPPs. Top: energy-dependent mechanisms which use ATP; Bottom (below black dashed line): proposed modes of spontaneous translocation of CPPs, including inverted micelle formation (bottom right), stable pore-forming mechanisms and non-pore-forming mechanisms; Non-pore-forming mechanisms can be distinguished into nondisruptive membrane permeabilization by accumulation of CPPs (carpet model, inset bottom left), or complete membrane disruption in a detergent-like model (inset bottom right). 
endocytosis-related mechanisms (or energy-dependent, in the sense of the cell investing energy to facilitate CPP uptake), or mechanisms of spontaneous translocation (or energy-independent). These mechanisms are globally illustrated in Figure 1.2.

The energy-dependent pathways consist of macropinocytosis and clathrin- or caveolin-mediated endocytosis (Figure $\mathbf{1 . 2}$ top left and right, respectively). Macropinocytosis is the non-selective uptake of liquid from the extracellular matrix, via actin-dependent enclosure[50]: dissolved components are thus taken up into the cell. This pathway has for example been associated with oligoarginine-modified lipid vesicles[51]. Clathrin and caveolin-mediated endocytosis are dependent on selective interactions with components on the plasma membrane. Clathrinmediated endocytosis is also called receptor-mediated endocytosis, as uptake begins with binding to specific receptors[52,53]. In the case of CPPs, this can be binding to negatively charged heparan sulfate by positively charged CPPs [54, 55]. This endocytotic mechanism involves coating of the endocytic vesicles with clathrin during the process of internalisation, hence the name "clathrin-mediated endocytosis". Caveolae-mediated endocytosis involves distinct glycolipid rafts[56], which are closely associated with caveolins, but do not involve clathrin-coating. This pathway has for example been associated with HIV-1 TAT[57].

The existence of mechanisms of spontaneous CPP translocation has been postulated after translocation was still observed in live cell experiments at $4{ }^{\circ} \mathrm{C}$ or otherwise lower temperature, where endocytotic pathways are assumed to be switched off $[21,32]$.

One of these proposed mechanisms includes the formation of an inverted micelle. The inverted micelle mechanism was simulated[58] but also suggested based on experimental results for penetratin[59]. It is assumed that binding of positively charged amino acid residues with negatively charged lipid bilayer components induces negative curvature in the plasma membrane, leading to an invagination. The driving force is minimisation of the peptide's potential energy, and it is proposed that this invagination spontaneously progresses towards inward vesicle budding.

Other energy-independent mechanisms are pore formation, a carpet model and detergent-like behaviour. These models are usually not discussed in detail in CPP reviews $[1,46]$, but are identical to some often-assumed mechanisms of action of 
antimicrobial peptides (AMPs) when they disrupt bacterial membranes (therefore a more detailed illustration of these mechanisms in the inset of Figure 1.2)[60, 61]. Pore formation is formation of a defined pore in the lipid bilayer (usually proven by membrane leakage experiments), The pore-forming mechanisms are distinguished into a barrel-stave model (inset Figure $\mathbf{1 . 2}$ top left) and a toroidal pore model (inset Figure $\mathbf{1 . 2}$ top right). In the barrel-stave model, the pore is solely formed by peptides, in multimeric helices. In the case of toroidal pores, the pore is formed by in-plane tilted lipids as well as peptides. The carpet model (inset Figure 1.2 bottom left) is a model of local and transient destabilisation upon extensive peptide binding, enabling the peptides to translocate. In the case of CPPs, this disruption is assumed to be transient, i.e. only temporary and non-toxic, whereas for AMPs, the disruption is intentionally toxic. AMPs are additionally assumed to be able to cause membrane disruption via a closely related detergentlike model (inset Figure $\mathbf{1 . 2}$ bottom right), which comprises membrane disruption by dissolution. This last model is generally undesirable for CPPs, as it is bound to cause cytotoxicity.

\subsubsection{Excursus: parallels between CPPs and AMPs}

The apparent functional parallels between CPPs and AMPs are not unnoticed in literature. In fact, they have been addressed in several reviews[26, 62-64], and have led to the creation of the general denotation of "membrane-active peptides" $[26,62,63]$. Several CPPs have also shown antimicrobial activity $[65,66]$ and vice versa $[67,68]$.

Just like CPPs, AMPs are often rich in arginine and lysine residues $[62,63]$. One well-known example is Magainin-II [69], GIGKFLHSAKKFGKAFVGEIMNS, derived from the Xenopus laevis frog skin. It has even been shown that for some CPPs, increasing their positively charged amino acid content increases their antimicrobial activity in parallel $[66,70]$.

The similarities between CPPs and AMPs, and possible explanations for this, have been explored experimentally by Wadhwani et al. [9]. They performed a liposome fusion assay with several well-known CPPs and AMPs, compared to an HIV fusion peptide FP13 as a positive control. The authors combined this with a physicochemical property analysis of employed peptides, as well as circular 
dichroism spectroscopy analysis in the presence and in absence of liposomes, to assess structural changes of the peptides. Surprisingly, no relationship was found between peptide charge or to which group the peptides belonged and their ability to induce liposome fusion. The most fusogenic peptides were those that were designed (not found in nature), both CPPs and AMPs. Fusogenicity correlated with a change in the peptides' circular dichroism signature when lipids were introduced, although no particular trends towards specific secondary structures could be observed. Wadhwani's work all in all showed that the most important parameter for activity prediction was structural change upon peptide binding. The importance of peptide secondary structure change upon membrane binding as a predictor for its CPP or AMP activity, had been emphasised before, by Almeida et al. [64]. Structural analysis has been recommended as a general experimental approach when assessing peptide function and making efforts at predicting their behaviour[63]. 


\subsubsection{Cell-entry mechanisms for arginine-rich CPPs}

Recently, two entry mechanisms specifically for arginine-rich or purely arginine CPPs were published $[10,11]$, both involving pore formation but with more nuance. The first was introduced in 2014 by Herce et al. [10]. Its proposal was prompted by the observation that uptake of several arginine-rich CPP (TAT $49-57$ and several arginine and lysine homo-oligomers) was enhanced when fatty acid content of the model cell membrane was increased. The proposed uptake scheme of these peptides mediated by fatty acids and a $\mathrm{pH}$ gradient across the plasma membrane, is depicted in Figure 1.3. First, arginine-rich peptides bind to deprotonated fatty acids outside the cell, through salt-bridge interactions ( $a$ to $b$ ). Then, fatty acids with peptides turn inside the membrane plane and form a transient "water channel" (c). Upon contact with the more acidic cytosolic environment, the CPPs would be displaced by protons while the CPP-fatty acid complex diffuses across the

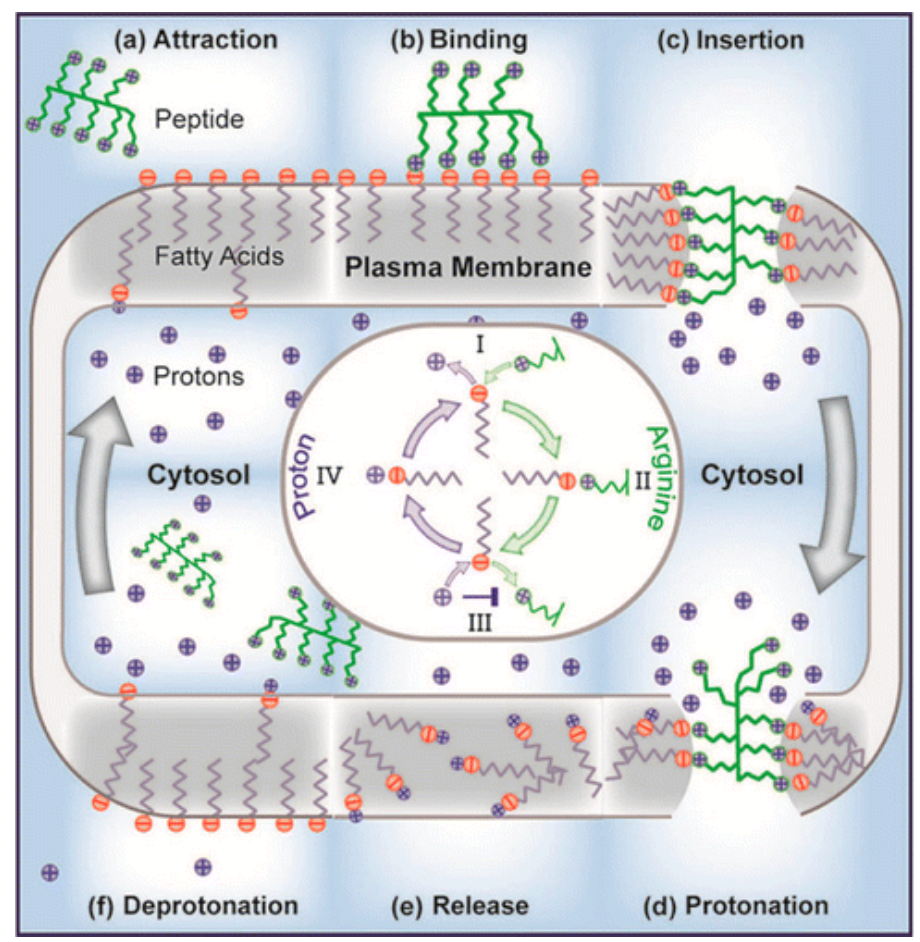

Figure 1.3: Proposed fatty acid- and $\mathrm{pH}$ gradient-mediated cell entry mechanisms for arginine-rich CPPs by Herce et al. [10]. a)-b) the arginine-rich residues are strongly attracted to negatively charged membrane fatty acids and bind; c) Membrane insertion occurs with formation of a 'water channel' and turning of fatty acids inside the bilayer plane; d)-e) Upon contact with the more acidic cytosol, protons compete with the arginine-rich peptide for binding and eventually the peptide is released into the cytosol; f) Fatty acids can freely move between the bilayer leaflets and are deprotonated upon contact with the more basic extracellular matrix. Figure reused from Herce et al. [10] with permission from ACS publications (https://pubs.acs.org/doi/10.1021/ja507790z). Further permission requests regarding reuse of this material have to be directed to ACS Publications. 
formed channel (d). Finally, the CPP is fully displaced by competing protons inside the cell and is thus released (e), and the channel closes. The fatty acids are able to move freely between the two bilayer leaflets, and get deprotonated once again in contact with the extracellular matrix (f). This $\mathrm{pH}$-mediated mechanism was further supported by the observation that when cells were incubated with labelled CPPs at lower $\mathrm{pH}$, uptake was almost non-existent. Simulations were performed to support this mechanism.

More recently, Allolio et al. [11] proposed a membrane fusion-based mechanism for spontaneous oligoarginine entry, based on simulations as well as vesicle experiments with nona-arginine $R_{9}$. It was experimentally supported by in giant vesicles in Figure $\mathbf{1 . 4}$ in which aggregation and fusion was observed, and ultimately involved CPP entry via formation of a fusion pore. The authors proposed that $R_{9}$ induces bifurcations of the membrane through strong adhesion and

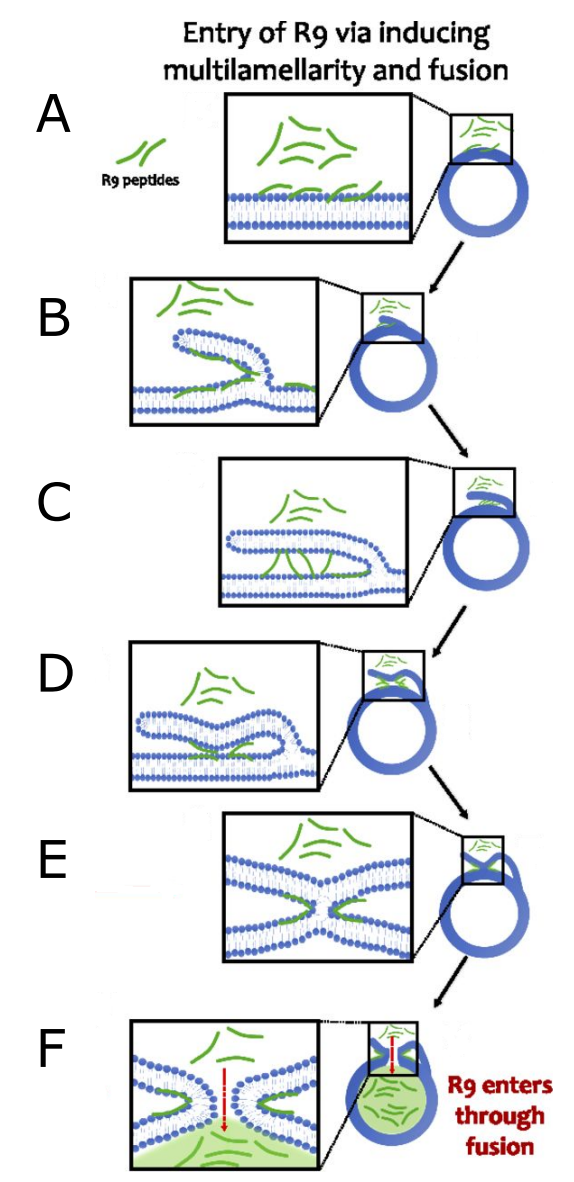

Figure 1.4: Mechanism of oligo-arginine entry via lamellarisation and fusion as proposed by Allolio et al. [11]. A: Rg binds tightly to the cell or vesicle membrane; $\mathbf{B}$ : Binding and curvature induction causes bilayer bifurcation to form; $\mathbf{C}$ : The bifurcation is extended and can be bridged to the main bilayer by the multiple guanidine moieties of $R_{9}$; $\mathbf{D}$ : The main bilayer and the bifurcation are brought closely together by $R_{9}$-bridging; $\mathbf{E}$ : This results in the formation of a hemifusion intermediate, which progresses into a fusion pore through which more peptides can enter the cell or the vesicle (F). Adapted from Fig. 2 in [11] with general permission from PNAS to reuse content for non-commercial purposes. 
curvature induction ( $\mathbf{A}$ to $\mathbf{B}$ ), which are bridged to the main bilayer by the multiple guanidine groups in the R-peptide (Figure 1.4 C). This bridging behaviour can overcome repulsive membrane-membrane interactions, thus bringing two membrane segments closer together (D) and ultimately causing membrane hemifusion (E) and formation of a full fusion pore (F), which allows entry of more CPPs into the vesicle or cell. This mechanism was further justified by simulation of $\mathrm{Ca}^{2+}$-induced fusion alongside $\mathrm{R}_{9}$-induced membrane fusion, and pointing out similarities between the two. Furthermore, upon addition to HeLa cells, domains of high $\mathrm{R}_{9}$-FITC fluorescence were observed, which were correlated with a multilamellar structure observed by electron microscopy. This structure was used as an argument why the formation of full-fledged fusion pores would be noncytotoxic, as they would be enclosed by this larger multilamellar structure and hence not cause actual cell content leakage.

The formation of such multilamellar particles on HeLa cells has been observed with FITC or otherwise labelled arginine-rich CPPs before (Hirose et al., 2012 [32]). They employed dodeca-arginine $\left(R_{12}\right)$ and other arginine-rich peptides. By performing correlative light and electron microscopy briefly after $\mathrm{R}_{12}$-addition to live cells, they correlated the brightly fluorescent particles (accumulation of peptide) to multilamellar structures on the bilayer. They further showed that $R_{12}-$ uptake depended on cargo covalently attached (hydrophobic cargo enhancing uptake), and that uptake depended on the plasma membrane potential. This led the authors to propose that this lamellarisation capability was part of longer Rpeptides' mechanism of entry, and that membrane potential as well as hydrophobic cargo played a role in this. Hence, lamellarisation of the cell membrane as part of CPPs' mechanism of entry is a principle that has been around for several years, but is only scarcely mentioned as such or researched in live cells. 


\subsection{Scope of the thesis}

CPPs are often rich in arginine and able to cross cell membranes without causing apparent damage. Several mechanisms by which this occurs have been proposed, as discussed above, and even multiple mechanisms can be used by the same peptide. Particularly the non-endocytotic mechanisms of cell entry mention membrane destabilisation, often in the form of pore formation, which is difficult to rhyme with the observation of generally low cytotoxicity for CPPs. Strong experimental evidence for any mechanism is difficult to obtain, since many events in the proposed mechanisms can hardly be directly observed experimentally.

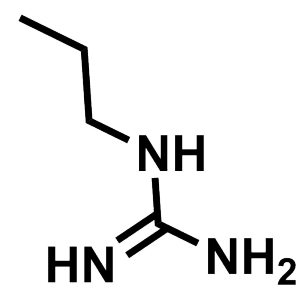

Figure 1.5: Chemical structure of molecular N-propylguanidine. A solution of protonated $\mathrm{N}$ propylguanidine is used as mimic of the arginine sidechain $\left(R_{\text {side }}\right)$ in this work.

Therefore, in this work, we investigated oligoarginine CPP interactions with a lipid bilayer via a bottom-up approach, investigating the intrinsic physical effects of the guanidine moiety on lipid bilayers by means of model systems of varying lipid headgroup composition. The effects of a guanidine-bearing mimic of the arginine sidechain (N-propylguanidine, denoted "Rside", Figure 1.5) were compared to the influence arginine oligopeptides $\left(R_{2}, R_{6}\right)$ have on the employed model systems.

Simulations of pore formation in the presence of arginine derivatives in different membrane lipid compositions were performed by Dr. Neha Awasthi. If arginine derivatives or oligopeptides should facilitate pore formation, that would be supportive of defined pore formation mechanisms of entry.

Additionally, in vitro experiments on membrane model systems of varying composition were performed by the author of this work. Pore formation simulations were emulated by breakthrough force spectroscopy experiments. Besides supporting simulation results, these experiments could also provide evidence for the membrane destabilisation component of several proposed mechanisms of spontaneous entry of CPPs: membrane destabilisation can be measured as a decrease of the force necessary to penetrate the membrane. Additionally, 
membrane destabilisation has also been explored by leakage assays in liposomes with the known CPP R6. Furthermore, fluorescence microscopy observations and fluorescence recovery after photobleaching on solid-supported lipid bilayers complemented the assessment of other physical effects of arginine derivatives on the membrane. An important observation with arginine oligopeptides, which led to the determination of dissociation constants for R-peptides of different lengths, will be addressed in a separate chapter (Chapter 5 Characterisation of $R_{6}$-induced domains, p. 91 ff.).

Two further intentions are served with our choice of experimental setups and model system composition. Firstly, with the use of different lipid compositions in our systems, we aim to identify interactions with specific lipid headgroups as key players in mechanisms of spontaneous cell entry for arginine-rich CPPs. The second deliberate choice is the use of unmodified arginine peptides, to avoid effects resulting from peptides attached to hydrophobic 'cargo' or markers[32, 41], as well as simple lipid compositions without the proteoglycans present on cell membranes. With this, our systems are truly a bottom-up approach at elucidating intrinsic interactions of arginine rich CPPs with lipid membranes, and can address the question whether such interactions play a role in $\mathrm{CPPs}^{\prime}$ mechanisms of cell entry.

Henceforth in this work, 'membrane' is used to indicate (components of) the experimental model systems used. This denotes 'lipid bilayer' and not any other membrane model system, unless clearly otherwise indicated. 



\section{MeTHODS BACKGROUND}

\subsection{Membrane model systems}

Lipid membranes are among the most important building blocks of all living cells. Not only do they form the barrier between cells and their environment, within the cells structures formed from membranes exist as well. These are denoted as 'organelles'[71]. Examples are the nuclear membrane in eukaryotic cells, as well as the double membrane of mitochondria and the complex folded structures of the Golgi apparatus and the endoplasmatic reticulum. Small spherical lipid membrane structures, called vesicles, facilitate transport of molecules between the organelles or out of or into the cell.

With their importance and versatility and therefore also complexity, influence of the lipid membranes themselves on biological phenomena has become difficult to study. Abundant proteins are integrated into lipid membranes to modulate their function or to be able to interact with other proteins or membrane structures. Furthermore, the cell membrane's behaviour is largely influenced by the attachment to the cytoskeleton, a dynamic structure of microtubules, actin fibrils and intermediate filaments that enables a cell to hold its shape and/or be mobile[71].

To be able to study the function of lipid membranes, often in concert with particular proteins, model systems for membranes have been developed. These lack the complexity of an entire cell and are often easier to maintain and reproduce experimentally. Widely used membrane model systems are vesicles/liposomes[72, 73] or solid-supported membranes (SSMs) [74, 75]. 


\subsubsection{Vesicular model systems}

Vesicular model systems are usually classified according to vesicle size or by lamellarity[73]. Small unilamellar vesicles (SUVs) are usually considered up to $50 \mathrm{~nm}$ in size. Large unilamellar vesicles (LUVs) range from 100-500 nm and giant unilamellar vesicles (GUVs) are typically $1 \mu \mathrm{m}$ in size or larger. Vesicles consisting of multiple lipid bilayers (lamellae) are multilamellar vesicles (MLVs).

Vesicles can be produced by several techniques, depending on what has to be incorporated into them and what the desired size is. Often, the first step is creating a dry film of lipids with their desired composition. This film is hydrated and suspended, to obtain a suspension of aggregates (MLVs). For SUVs, treatment with strong ultrasound pulses ('sonication') is a widely used method, as it is straightforward and uncomplicated. If proteins have to be incorporated into the membrane of SUVs, the detergent dilution method[76] is more desirable. In this method, a mixture of lipids and proteins is dissolved together with a mild detergent to form a micellar mixture. The detergent is then removed by gel filtration. A method to create larger vesicles with a defined size population, is extrusion[73]: a suspension of lipids is extruded through a polycarbonate membrane with a defined pore size. For giant unilamellar vesicles, more complex procedures, such as electroformation[77], come into play.

Vesicle model systems have been widely applied to assess the function of proteins[73]. A widely studied phenomenon is that of membrane fusion[78, 79], often with the purpose of vesicles to serve as drug carriers. Furthermore, vesicles are usually employed as model membrane in spectroscopic methods which require a liquid sample, such as fluorescence spectroscopy and circular dichroism spectroscopy[72]. Another application of vesicles, is assessment of the ability of compounds to disturb a cell membrane, via leakage assays[72].

In leakage assays, the vesicles are typically filled with a dye whose fluorescence is quenched when inside the vesicles. If the integrity of the vesicles is compromised, the dye will leak out and is thus diluted, which makes its fluorescence increase. Fluorescence intensity of the dye is hence used as a measure for leakage. Normalisation for $100 \%$ leakage is usually performed by destroying all vesicles with a detergent. Dyes or dye-ligand pairs that are often used for a leakage assay are ANTS/DPX[80], calcein[81], carboxyfluorescein[82] 
and sulforhodamine B[83]. In case of ANTS/DPX, DPX quenches the fluorescence of ANTS while in the vesicles. ANTS is released upon leakage from vesicles, hence its fluorescence increases. All other dyes mentioned are self-quenching at higher concentrations.

\subsubsection{Solid-supported membranes}

Solid supported lipid membranes usually are lipid bilayers spread on a solid surface[74, 75]. Bilayers can be formed on solid substrates in several configurations[74, 75]: directly supported by a hydrophilic substrate, as monolayer, as tethered bilayer, as bilayer supported by a polymer cushion (often polyethylene glycol (PEG))[84], or as partially free-floating bilayer, for example on a porous substrate [85-87].

Solid substrates mostly consist of hydrophilized, polished planar materials of various kinds. Many used materials are silicon-based, such as glass, silicon wafers with a silicon oxide layer, silicon nitride, or mica. Also alumina[85] has been employed as solid support. Recently, oxidised polydimethylsiloxane came up as a direct support for lipid membranes and not as a cushion. It has the advantage of deformability, opening possibilities for membrane stretching[88, 89]. Examples of applications of non-planar solid supports are wavy glass substrates[90] and glass beads[91, 92].

SSMs can be obtained by three main methods[74, 75]. The first is the use of a Langmuir-Blodgett trough[93], consisting of a lipid monolayer situated at an airwater interface, at a controlled pressure. By submerging the intended solid support into the liquid, a monolayer can be attached to the support. Multiple submersions lead to multiple lipid bilayers. The lipid-covered substrate can then be hydrated with the desired buffer and used for experiments. The second method that is widely used, is the direct spreading of vesicles onto the hydrophilic substrate, in liquid $[94,95]$. It is often aided by introducing osmotic imbalance between the vesicle exterior and interior. The last method is bilayers formed directly from organic solvent by spin-coating[96, 97]. A drop of organic lipid solution is disposed on the solid substrate and by rapid spinning the solvent is evaporated. Thus, the lipids are evenly distributed over the solid substrate. This method usually yields membrane stacks. 


\subsection{Atomic force microscopy}

Atomic force microscopy (AFM) has become a versatile tool in biophysics, surface science and material science due to its high precision. Since its invention in 1986 [98], its principle has been generally applied to 1) measure forces between surfaces and even molecules 2) manipulate surfaces at the nano-level and 3) obtain high resolution topography images. In the fields of surface science and nanotechnology, it has been applied to characterise surface roughness and differentiate between different materials on a surface, as well as imaging of crystalline surfaces with atomic resolution[99]. Furthermore, force spectroscopy has been applied in the creation of nanoscale devices (mostly nanorods) consisting of various materials[99]. In biology and the biomedical field, AFM has also been applied for the imaging of structures, such as viral particles, cells, or even protein complexes[100]. Force spectroscopy has provided information on for example the nano-mechanics of cells[101], as well as their adhesion to surfaces[102] and their mechanical response to knock-out of proteins[103] or the addition of drugs.

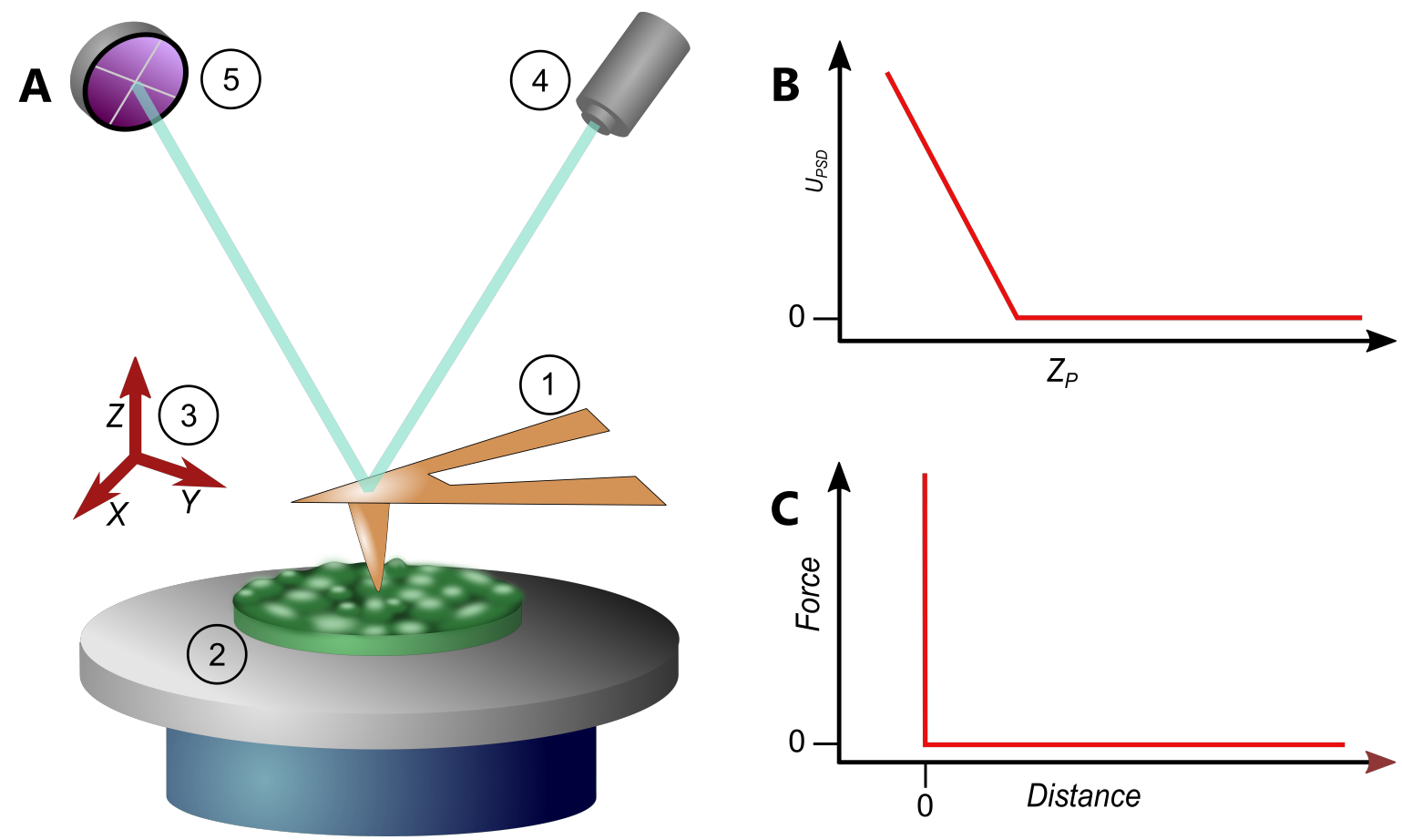

Figure 2.1: Principles of atomic force microscopy. A: Schematic AFM setup with (1) a cantilever that is moved over a sample (2) and controlled by piezoelectric elements in all directions (3); A laser beam (4) is reflected on the back of the cantilever, onto a position-sensitive detector (PSD, (5)). B: Illustration of an approach force curve measured on an incompressible sample without any processing; force is 0 when not in contact with the sample; $\mathbf{C}$ : A force-distance curve obtained after cantilever calibration and determination of the contact point: the position detector signal (force) after the contact point is set to zero distance. 
Specifically in solid-supported lipid bilayers, AFM has provided information on phase separation of lipids by imaging as well as force spectroscopy (recording of force curves by approaching and retracting a cantilever on a sample and measuring its deflection)[94]. Furthermore, differences in mechanical properties between different membrane components has been shown, as well as responses of these mechanical properties and membrane thickness to changes in experimental conditions, such as temperature[104-106] and the presence of different ions in solution[107-111].

The concept of AFM can be described by five components (Figure 2.1 A, p. 30): A small flat spring probe with a sharp tip and a reflective back surface (a cantilever, (1)) is moved over a sample (2). The probe movement is controlled by piezoelectric elements in $\mathrm{X}-, \mathrm{Y}$ - and Z-direction (3). The Z-direction piezoelectric element is usually in connection with the cantilever (in the AFM 'head'). The $X, Y$ piezoelectric element can be either situated in the sample stage or together with the Z-direction element in the AFM head. Cantilever movement is detected by a laser beam (4) directed at the cantilever's back side, which is then reflected onto a positionsensitive detector (PSD, (5)). This technique is called the optical lever technique.

The cantilever can be deflected through forces exerted on it. These forces can either be applied by the sample via the tip of the cantilever, or by the environment due to its viscosity (such as hydrodynamic drag $[112,113]$ ). Cantilever deflection normally follows Hooke's law for a simple harmonic spring:

$$
F=k_{\mathrm{c}} * s
$$

in which $F$ is the force, $k_{\mathrm{c}}$ is the cantilever's spring constant and $s$ is the cantilever deflection. The spring constant $k_{\mathrm{c}}$ can theoretically be calculated from the cantilever's dimensions, the Young's modulus of its composing materials and the mass of the tip. However, the exact dimensions and composition of individual cantilevers is usually not known. Furthermore, a thin layer of material can exhibit different physical properties than its bulk, having consequences for predicting the behaviour of coated cantilevers. Therefore, several methods have been developed to experimentally calibrate cantilevers, such as using a cantilever with known spring constant for calibration, attaching a known mass to the cantilever and the Sader method which uses only thermal noise data[114]. 
The cantilever calibration method that is used by most AFM users today and which is generally integrated into AFM control software, is a combination of obtaining one force curve and fitting the thermal noise data. With the force curve on a hard surface (stiffness much higher than cantilever stiffness), the relationship between Z-piezo displacement and PSD-voltage ( $U_{\text {PSD }}$ ) is determined by determining the slope of the non-horizontal part of the curve (Figure 2.1 B p. 30, horizontal means no change in deflection and hence no contact). Geometrically, Z-piezo displacement is near identical to cantilever deflection if the tip is in contact with a hard surface, provided deflection is not too great[115]. Subsequently, a spectrum of the thermal oscillation of the cantilever is obtained, by Fourier transforming the thermally fluctuating position detector signal from the cantilever. By fitting a Lorentzian curve to the fundamental resonance frequency part of this 'frequency spectrum' and subsequently integrating this function, one can obtain the mean square spring displacement in the main resonance mode, $\left\langle q^{2}\right\rangle$. Via deduction from the Hamiltonian for a harmonic oscillator in equilibrium with its surroundings and using the equipartition theorem, one can obtain the spring constant $k_{\mathrm{c}}$ via

$$
k_{\mathrm{c}}=\frac{k_{\mathrm{B}} T}{\left\langle q^{2}\right\rangle}
$$

where $k_{\mathrm{B}}$ is the Boltzmann constant $\left(1.38064852 \times 10^{-23} \mathrm{~J} / \mathrm{K}\right)$ and $T$ is the temperature in Kelvin. This procedure was described by Hutter et al. [116] and refined by Butt \& Jaschke [117].

The data of force versus piezo-signal can be converted in to force-distance curves when the cantilever has been calibrated contact point has been established: from the contact point onwards, the distance from the sample is defined as 0 (Figure 2.1 C). Determination of the contact point is usually done during data processing, since this is not always straightforward and depends on user's needs (see below).

The AFM can be operated in several modes: contact mode, non-contact mode, intermittent contact mode and several more. In this work, only contact mode is used, hence the other modes will not be explained further. Contact mode will be further explained with the help of breakthrough force spectroscopy. After this, QI ${ }^{\mathrm{TM}}$ mode of imaging will be briefly explained, as it is used for membrane topography imaging in this work. 


\subsubsection{Breakthrough force spectroscopy}

Figure 2.2 shows a schematic approach and retract force-distance curve, along with illustrations of what happens to a lipid bilayer at each phase of the force curve during which the cantilever tip breaks through the membrane. During approach (red), the cantilever is at first not in contact with the membrane (1), hence there is no net deflection of the cantilever. When the cantilever starts deflecting, at some point, the tip is in contact with the sample ((2), usually defined as the point from

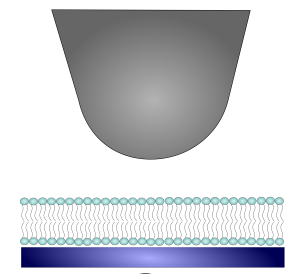

(1)

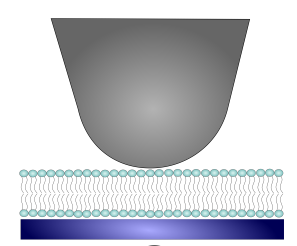

(2)

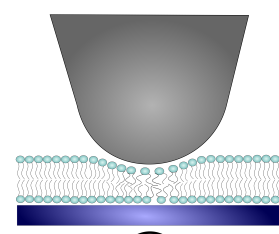

(3)

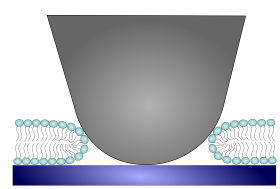

(5)

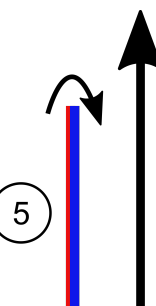

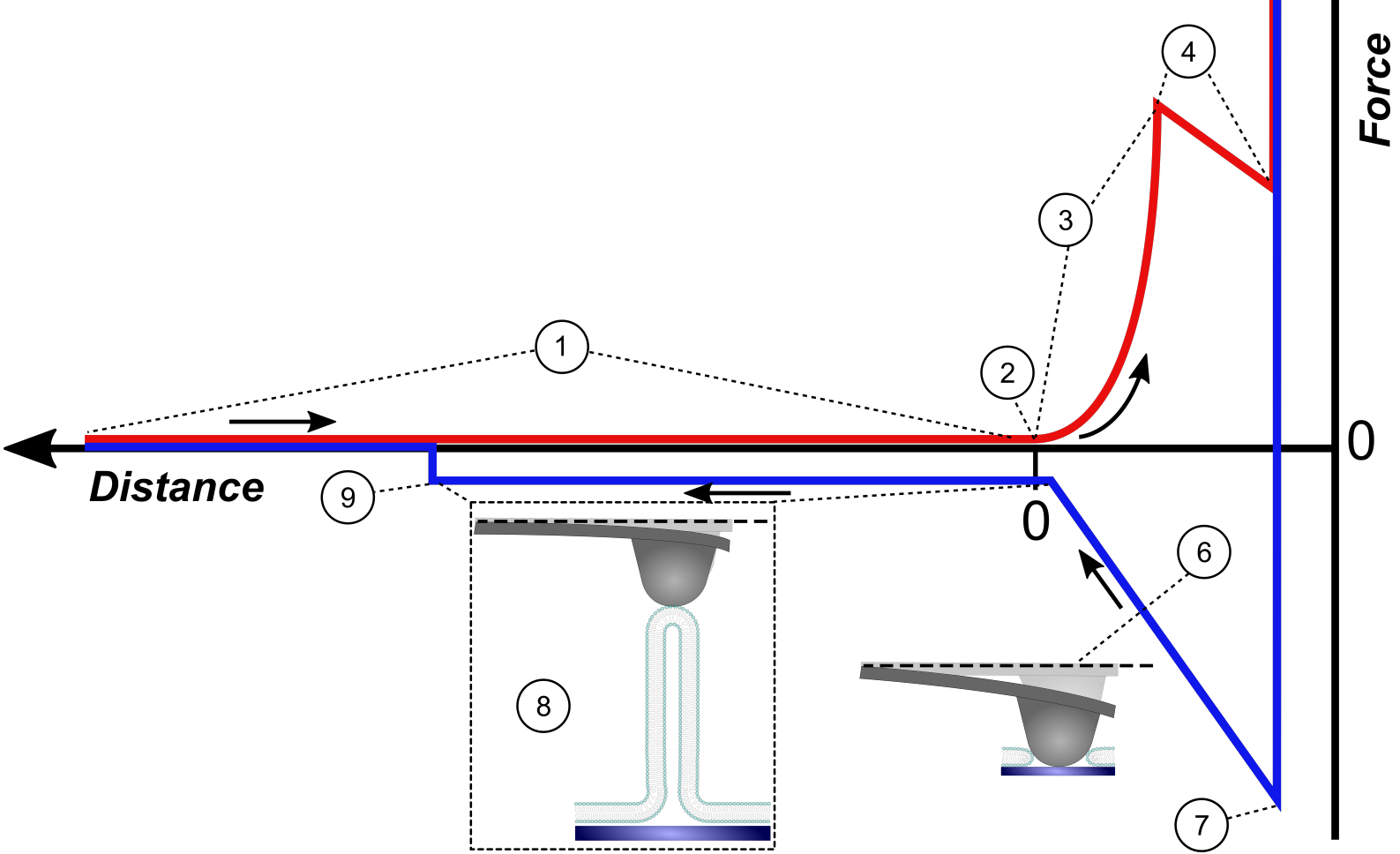

Figure 2.2: Illustration of an approach (red) and retract (blue) force vs. distance curve with a breakthrough event. Sections are labelled with numbers 1-9 and accompanying illustrations are labelled with the same number. (1) As the cantilever approaches, its deflection is initially zero. The contact point (2) in breakthrough experiments is usually defined as the point from which cantilever deflection starts increasing upon approach. The cantilever tip deforms the membrane as it exerts more force (3), until at some point it breaks through with a discrete event (4). After breakthrough, the cantilever is in contact with the solid support (5). As the Z-piezo retracts further, it is often the case that the cantilever tip adheres to the solid substrate, resulting in a negative deflection (6). When the cantilever detaches, there is a discrete turn in the deflection, often termed the 'adhesion peak' (7). It is also possible that the membrane adheres to the cantilever and that a tube-like structure is pulled from the surface as the cantilever retracts (a tether (8)), which results in a constant 'tether force'. The tether eventually detaches via a discrete jump in the retract curve (9), and the deflection returns to zero. 
which cantilever deflection starts increasing in case of SSMs, see below for theoretical background). As the force exerted by the cantilever increases, the membrane is indented (3), and at a critical force, defined as the breakthrough force or yield force, membrane lipids get displaced during a discrete event: the breakthrough (4). After breakthrough, the cantilever tip is in contact with the solid support (5) until a certain pre-set force (the setpoint) is reached.

Subsequently, the cantilever is retracted again (blue). It is possible that adhesion takes place between the cantilever tip and the solid substrate, which results in negative deflection of the cantilever after it passes the point at which contact initially occurred during approach (6). If the measurement of interest involves mainly the retraction curve, the point of zero deflection during retraction is usually defined as the contact point. Eventually, the force exerted by the cantilever overcomes the adhesion force and a discrete detachment event is observed (7), often informally termed the 'adhesion peak'. After detachment from the solid substrate, it is possible that the membrane still adheres to the cantilever tip, and a minimal tubular structure (tether) is lifted off from the substrate (8). It was demonstrated that this results in a constant force consisting of two components[87]: a term to describe how much force it takes to lift lipids from the solid substrate, which is always the same, and a membrane tension term. The latter is also constant since the expansion by tether pulling is usually negligible in comparison to the total membrane surface area. Finally, when the cantilever is retracted far enough, the tether detaches, resulting in a discrete step in the retraction curve (9) and the deflection (detected force) returns to zero.

\subsubsection{Theoretical background: contact regime}

\section{Contact point and contact regime}

As mentioned before, the determination of the contact point is not always straightforward. As Butt described[115], there are three situations where the contact point can be easily set. The first one is a linear force curve with a sharp kink, when both sample and cantilever tip are non-deformable and there are no surface forces (interactions between tip and sample other than contact) present, as shown in Figure 2.1 B and C (p. 30). The second one also describes nondeformable materials, but with repulsive surface forces: contact is established for the point from which the force curve is linear. The third situation involves no 
surface forces but a deformable sample: the contact regime is non-linear, but because of the absence of surface forces, the contact point can be defined from the point where the cantilever deflection increases. Realistic samples, including the solid-supported lipid membranes in this work, are however often a combination of aforementioned situations: surface forces and deformability. Defining the exact point of contact in biophysical experimental setting is therefore challenging, because the surface forces can cause deformation before contact is actually achieved. Additionally, both cantilever tip and sample can exhibit surface roughness, which can lead to inconsistent contact determination.

This combination of deformability and different kinds of forces between tip and sample also has consequences for the interpretation of the force curve progression once contact is established. For the case that the sample is fully elastic, meaning it recovers its original shape after cantilever indentation, at least three models defining the contact regime in terms of the contact area and the indentation depth $\delta$ have been established. The first is the Hertz-model[118], which assumes there is no adhesion between the cantilever tip and the sample. The Johnson-KendallRoberts (JKR)[119] and the Dejarguin-Müller-Toporov (DMT)[120-122] model do assume adhesion forces, inside and outside the contact regime, respectively.

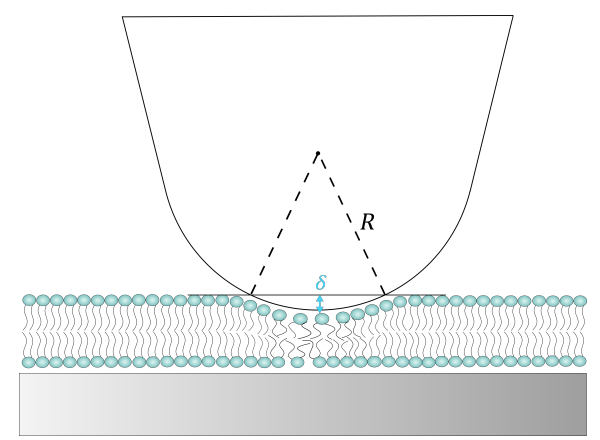

Figure 2.3: Illustration of cantilever tip radius $R$ and indentation depth $\delta$.

All of these models demonstrate that the interaction of the cantilever tip and the sample is dependent on cantilever tip radius $R$ (see Figure $\mathbf{2 . 3}$ ). The Hertz-model, for example, defines the contact radius $a$ as

$$
a=\sqrt[3]{\frac{R F}{E_{\text {tot }}}}
$$

where $F$ is the force exerted by the cantilever and $E_{\text {tot }}$ a reduced Young's modulus for both tip and sample. It can be deduced that the larger the tip radius $R$, the 
larger the contact area, which is intuitive. Furthermore, the indentation depth $\delta$ is given by

$$
\delta=\frac{a^{2}}{R}=\sqrt[3]{\frac{F^{2}}{R E_{\mathrm{tot}}^{2}}}
$$

Hence it can also intuitively be deduced that for the same exerted force but a larger tip radius, penetration into the sample is less deep. Intuitively assuming that a certain penetration depth has to be reached before breakthrough occurs, it can thus be deduced that the breakthrough force detected varies with cantilever tip radius. This was indeed experienced in this work (see 4.2 Breakthrough force analysis p. 64) and has recently been systematically shown in literature[123].

\section{Breakthrough process theory}

The second theoretical background related to this work, is two theoretical models for the process of indentation and breakthrough through thin molecular films, developed by Butt and Franz[124]. Both proposed models view breakthrough as an activated process: an energy barrier has to be overcome before breakthrough occurs. This energy barrier is lowered when the pressure of the cantilever tip on the sample is increased. The first model, denoted the 'continuum nucleation model', assumes the film molecules to be equivalent springs that to not experience shear between one another. By deducing the energy of a hole between cantilever tip and molecular film, the authors came to the following expressing for the activation energy for this model:

$$
\Delta U=\frac{2 \pi^{2} \Gamma^{2} R}{F-2 \pi R S}
$$

where $\Delta U$ is the activation energy, and $F$ the force applied at any given point. Hence, the activation energy decreases as the force increases. $R$ is still the cantilever tip radius, and $\Gamma$ is the line tension, and $S$ is the so-called spreading pressure. Line tension is a measure for cohesion between the film molecules, while spreading pressure $S$ is a measure to express the tendency of the film molecules to adhere to the solid substrate.

The second model, denoted 'molecular model', assumes that the thin film consists of molecules with binding sites that can be occupied. The nature of this occupation is determining for the energy of the molecules. The cantilever tip pressing onto 
the molecular film increases the energy of the molecules, by causing a pressure gradient. By deducing the compression energy of a molecule with the help of their elastic energy and average distance between molecules, equation 2.5 for the activation energy in a simplified version of the molecular model.

$$
\Delta U=\Delta U_{0}-\frac{\alpha V F}{2 \pi h R}
$$

Here, it is even more clearly shown that activation energy is decreased with applied force: $\Delta U_{0}$ is the initial activation energy, and a term containing the applied force is subtracted from it. $\alpha$ is a geometrical factor, which should be 0.5 unless the cantilever tip is of the same size order of the film molecules. $h$ is the molecular film's thickness and $V$ is the so-called 'activation volume' for $x$ number of molecules under the tip, given by $V=2 x \lambda^{2} h$ for a double layer of molecules.

Both models lead to expressions for the probability distribution function of the breakthrough force that contain the approach rate of the cantilever towards the sample. The continuum nucleation model also incorporates a frequency factor for breakthrough 'attempts', which should be equal to the fundamental resonance frequency of the cantilever. The probability distribution derived from the simplified molecular model contains a thermal force and a rate constant for the spontaneous formation of holes.

Loi, Butt \& Franz[125, 126] as well as Künneke, Krüger \& Janshoff[127] demonstrated that both models can be applied to breakthrough force data on lipid bilayers and demonstrate differences in their properties. Overall, the continuum nucleation model therefore yields parameters that describe collective mechanical behaviour of membrane molecules, such as cohesion and adhesion to the substrate. The molecular model concentrates on energy of the membrane molecules and gives an idea of this energy dependent on temperature, related to the breakthrough forces observed.

\subsection{3 $\mathrm{QI}^{\mathrm{TM}}$ mode for imaging}

Quantitative imaging mode ( $\left.\mathrm{QI}^{\mathrm{TM}}\right)$ as developed by JPK Instruments is a form of fast force-distance curve acquisition. For every pixel in a recorded image, one force-distance curve is recorded with low force setpoint. The exact control of the cantilever is not disclosed by JPK instruments. However, algorithms developed by JPK provide possibilities to obtain not only surface topography, but also analysis 
of individual force curves, hence providing access to mechanical parameters of the sample if so desired.

\subsection{Reflectometric interference}

\section{spectroscopy}

Dynamic reflectometric interference spectroscopy (RIfS) utilizes the differences in refractive indexes of different materials in order to determine the thickness of thin films of a material. If light falls on an interface between two different materials, it can be transmitted or reflected. The differences in refractive properties of multiple materials on top of each other, make that at each interface, the light is refracted, dependent on each interfacing material's refractive index. At the same time, part of the light is reflected at each interface, resulting in multiple beams of reflected light with all different path lengths and which are therefore phase shifted with respect to each other (illustrated in Figure 2.4 A) [128, 129]. This results in an interference pattern, that is different for each wavelength of light and hence results in a reflection spectrum over multiple wavelengths that resembles a sinusoidal wave (Figure 2.4 B) [128, 129].
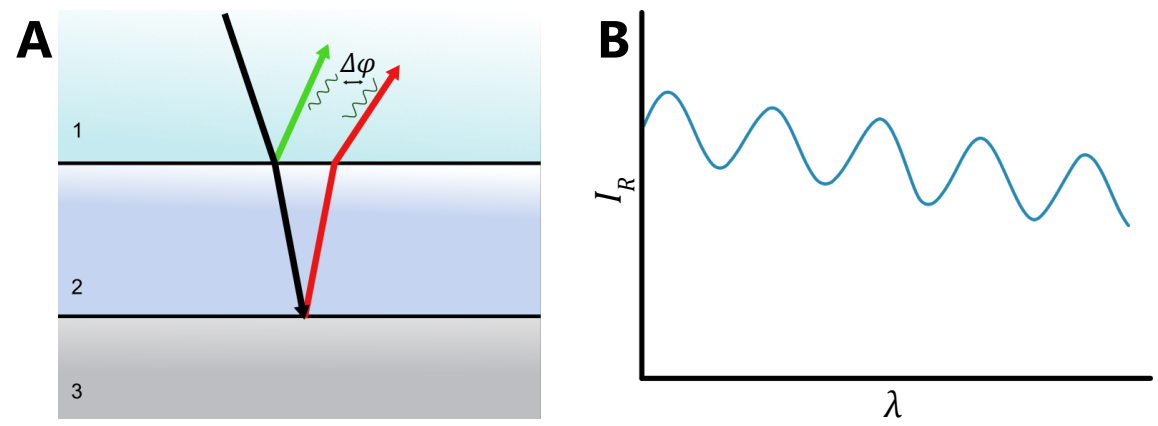

Figure 2.4: Concepts of reflectometry. A: Illustration of the fate of an incoming light wave, diffracted or reflected by three materials: at each material interface, partial reflection takes place. The detected light is hence a combination of at least two reflected waves with different path length and a phase shift $\Delta \varphi$, resulting in interference. B: Illustration of a reflection spectrum as typically obtained from RIfS, after correction for a spectrum showing full reflection (polished aluminium). Interference is different at each wavelength, resulting in an intensity $I_{R}$ vs $\lambda$-graph that resembles a sinusoid.

We will illustrate how quantitative optical thickness data can be deduced by an example with three layers of materials, with their refractive indices $n_{1}, n_{2}$ and $n_{3}$. At an interface of material $i$ and $j$, the Fresnel coefficient $r_{i j}$ can be calculated[130]: 


$$
r_{i j}=\frac{n_{i}-n_{j}}{n_{i}+n_{j}}
$$

If light is only reflected on the interfaces between materials 1 and 2 and between material 2 and 3, making two evanescent beams of light, the measured intensity of reflected light $I_{R}$ with interference pattern can be calculated as:

$$
I_{R}(\lambda, \Delta \varphi)=\frac{r_{12}^{2}+r_{23}^{2}+2 r_{12} r_{23} \cos (2 \cdot \Delta \varphi)}{1+r_{12}^{2} r_{23}^{2}+r_{12} r_{23} \cos (2 \cdot \Delta \varphi)}
$$

with phase shift $\Delta \varphi$ between the two reflected beams at a given wavelength $\lambda$. This can be precisely calculated if refractive indices of all materials are known. The phase shift at any given wavelength is given by:

$$
\Delta \varphi=\frac{2 \pi}{\lambda} O T
$$

where $O T$ is the optical thickness of material 2 given by $O T=n_{2} d_{2}$ with $n_{2}$ as the refractive index of material 2 and $d_{2}$ the physical thickness of material 2. Knowing this, equation (2.7) can be reformulated to:

$$
I_{R}(\lambda, O T)=\frac{r_{12}^{2}+r_{23}^{2}+2 r_{12} r_{23} \cos \left(\frac{4 \pi}{\lambda} \cdot O T\right)}{1+r_{12}^{2} r_{23}^{2}+r_{12} r_{23} \cos \left(\frac{4 \pi}{\lambda} \cdot O T\right)}
$$

Hence, if all refractive indices of involved materials are known, changes in optical thickness can be calculated from a reflection spectrum by fitting equation (2.9) (or an adapted form for more materials) for each measured wavelength[128, 130].

If optical thickness changes due to for example adsorption of small molecules in a dynamic experiment, the spectrum of $I_{R}$ shifts to the left or right, with a wavelength step $\Delta \lambda$ that is proportional to the optical thickness change[131,132]. Hence, after fitting the complete spectrum, the intensity value for one wavelength is typically monitored over time, and transformed into optical thickness change with respect to the optical thickness measured when the spectrum was first fitted.

\subsection{Fluorescence microscopy}

Fluorescence microscopy is a useful tool to observe specific structures in for example cells, by specifically labelling them with a fluorescent dye. The dye is 
selectively illuminated and its emitted light is selectively detected. This also enables the direct observation of structures that are naturally colourless and only scatter light, such as membranes.

In fluorescence microscopy, the illumination light (usually a halogen or tungsten white light source) is passed through a filter cube[133]. This filter cube contains a filter for the excitation light, which is then directed onto the sample by a 45degree-tilted dichroic mirror. The dichroic mirror is designed to reflect the excitation light, but transmit other wavelengths. The light from the sample is passed through an emission filter onto a detector.

A special form of fluorescence microscopy which improves resolution by limiting the detection of light outside the focal plane, is confocal laser scanning microscopy[134, 135]. Its principle is schematically depicted in Figure 2.5. The light source is a laser of specific wavelength, which scans the desired imaging area pixel by pixel. This already improves resolution with respect to conventional fluorescence microscopy, since only a very small area of sample is illuminated. Additionally, the emission light is directed through a small aperture, denoted a 'pinhole'. The pinhole ensures that only light stemming from emission sources close to the set focal plane passes onto the detector. Light from other focal depths will not pass the pinhole, and therefore will not cause blur in the detected image.

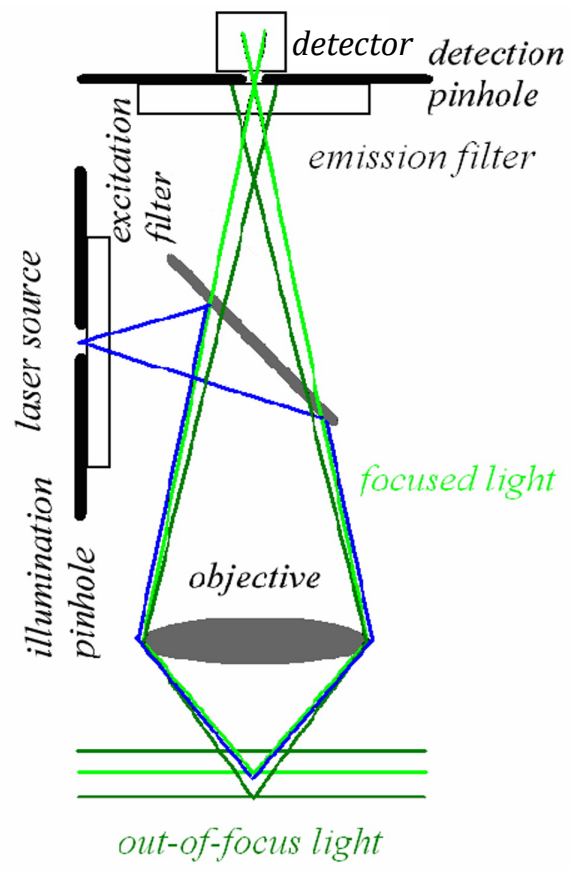

Figure 2.5: Principle of confocal laser scanning microscopy. Light too far outside the focal plane does not pass the detection pinhole. Illustration adapted from [133] under the Creative Commons 2.0 license. 


\subsection{Simulations of pore formation}

Molecular dynamics simulations of pore formation were performed by Dr. Neha Awasthi (supervised by Prof Dr. Jochen Hub, Computational biophysics group, Saarland University). Therefore, they will be introduced in brief. Details have been outlined in [136], [137] and [138].

Pore formation in lipid bilayers is often simulated along a particular reaction coordinate, meaning the way in which pore formation in the simulated bilayer is induced. This is done with the purpose of determining the free energy of pore formation. In this work, the reaction coordinate is the number of water molecules present in a transmembrane cylindrical region [139](Figure 2.5 B). As more and

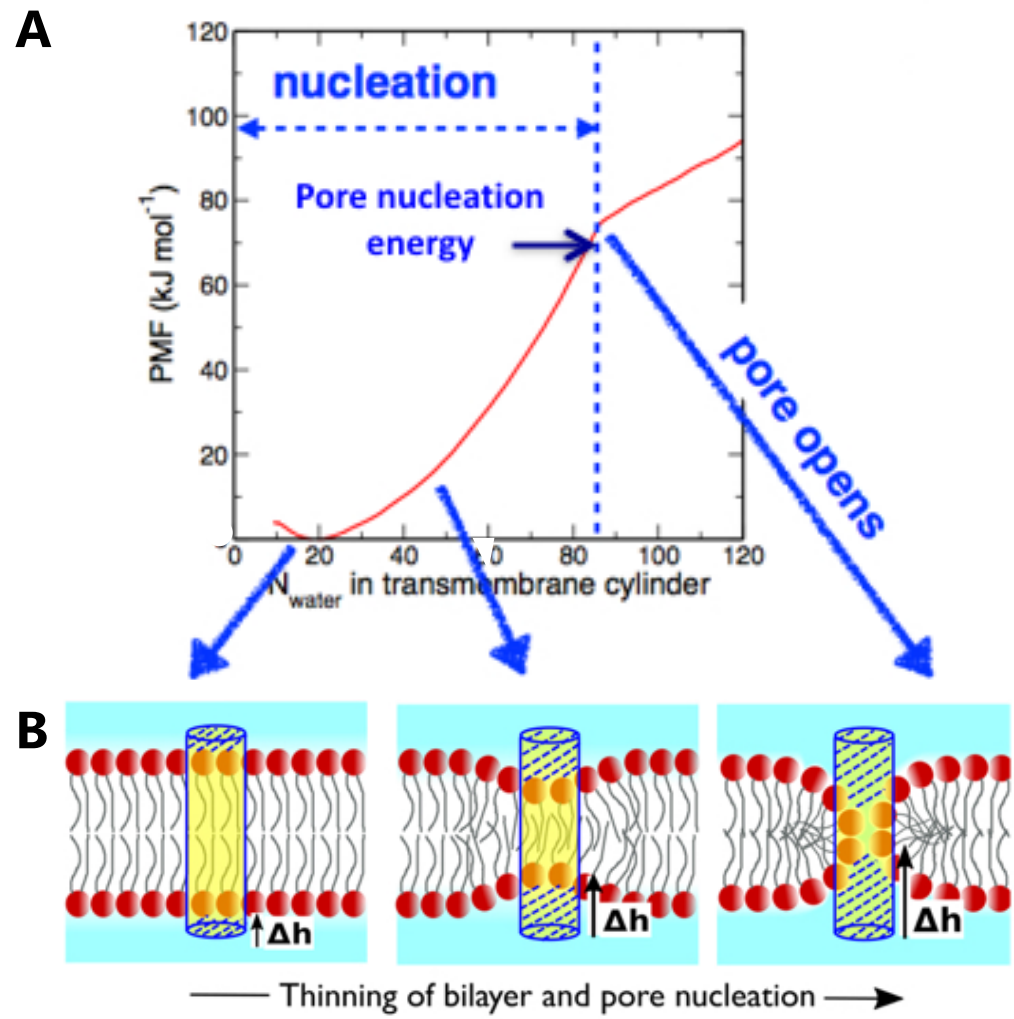

Figure 2.6: Illustration of the deduction of pore nucleation energy from MD simulations of pore formation by Dr. Neha Awasthi, adapted with permission. A: A typical PMF as a function of number of water molecules $N_{\text {water }}$ in the transmembrane cylinder; B: Graphical illustration of what happens to the membrane if more water molecules enter the transmembrane cylinder, related to the different regimes of PMF as a function of $N_{\text {water }}$ in the transmembrane cylinder. 
more water molecules are forced into the transmembrane cylinder, eventually a pore opens.

The free energy surface along the reaction coordinate is called the 'potential of mean force' (PMF) [140, 141]. An example of a PMF along the number of water molecules in the cylindrical region is shown in Figure 2.5 A. Before pore formation, a reduction in membrane thickness takes place, defined as $\Delta h$. The PMF can be fitted to a quadratic function before pore opening, and from that a membrane thinning modulus can be deduced. Pore opening takes place at the kink in the PMF. This kink is regarded as the potential energy of pore formation, as derived from these simulations. When this kink occurs at lower PMF or at less water molecules inside the transmembrane cylinder, this indicates that pore formation is easier. Other physical parameters, such as membrane thickness and area per lipid, are calculated for each simulation at the minima of the PMF, which corresponds to the intact membrane. 


\section{MATERIALS \& METHODS}

All preparative procedures and experiments in this work were carried out at room temperature, unless indicated otherwise.

\subsection{Materials}

\subsubsection{Buffers}

All buffers were prepared using ultrapure water with a resistivity of $18.2 \mathrm{M} \Omega \mathrm{cm}$, as obtained from a Milli-Q Advantage A10 Ultrapure Water Purification System equipped with a MilliPak ${ }^{\circledR}$ Express 40 filter with $0.22 \mu \mathrm{m}$ pore size. Demineralised water fed to the Milli-Q system was pre-processed by an Elix® Reference 5 system. All abovementioned devices were produced by Merck Millipore.

After dissolving the materials for the buffer, buffers were filtered over a $0.2 \mu \mathrm{m}$ pore size cellulose acetate filter (Sartorius Stedim Biotech) and degassed right after with the help of a Nalgene ${ }^{\mathrm{TM}}$ polysulfone bottle top filter device (Thermo Fisher Scientific, Schwerte, Germany). After filtration, $\mathrm{pH}$ of the buffers was adjusted to the desired value using $1 \mathrm{M} \mathrm{HCl}$ solution (diluted with ultrapure water Table 3.1: Buffer compositions used in this work.

\begin{tabular}{ccc} 
Buffer name \& pH & Component & Concentration / mM \\
\hline Spreading buffer & Sodium citrate dihydrate & 20 \\
$\mathrm{pH} 4.8$ & $\mathrm{KCl}$ & 50 \\
& EDTA sodium salt & 0.1 \\
& dihydrate & \\
& $\mathrm{NaN}_{3}$ & 0.1 \\
\hline Measuring buffer & $\mathrm{Na}_{2} \mathrm{HPO}_{4} \cdot 2 \mathrm{H}_{2} \mathrm{O}$ & 50 \\
pH 6.8 & EDTA sodium salt & 0.1 \\
& dihydrate & \\
\hline
\end{tabular}


from concentrated $\mathrm{HCl}$ solution) or $1 \mathrm{M}$ sodium hydroxide solution (obtained by dissolving $\mathrm{NaOH}$ pellets). All buffer compositions used in this work are detailed in Table 3.1. 'Spreading buffer' with citrate was used to obtain solid supported lipid bilayers. 'Measuring buffer' with $50 \mathrm{mM}$ phosphate was used for nearly all experiments.

\subsubsection{Lipids}

1-Palmitoyl-2-oleoyl-sn-glycero-3-phosphocholine (POPC), 1-palmitoyl-2-oleoylsn-glycero-3-phospho-(1'-rac-glycerol) (sodium salt, POPG), 1-palmitoyl-2oleoyl-sn-glycero-3-phosphoethanolamine (POPE) and 1-palmitoyl-2-oleoyl-snglycero-3-phospho-L-serine (sodium salt, POPS) were bought as $1 \mathrm{mg} / \mathrm{ml}$ solutions

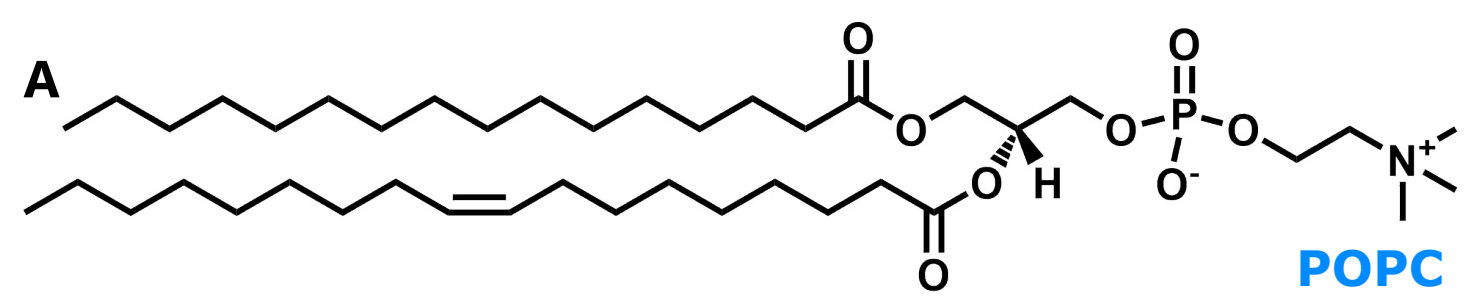<smiles>[B]CCCCCCCCCCCCCCCCCCCCCCC(=O)OC[C@H](COP(=O)(O[NH3+])OCC(O)CO)OC(=O)CCCCCCCC</smiles><smiles>[2H][C@@H](COC(=O)CCCCCCCCCCCCCCCCCCCCCCCC)COP(=O)([O-])OCC[NH3+]</smiles><smiles>[2H][C@H](COC(=O)CCCCCCCCCCC/C=C\CCCCCCCC)COP(=O)(O[NH3+])OC[C@H]([NH3+])C(=O)[O-]</smiles>

Figure 3.1: Lipids used for model membranes in this work. A: 1-Palmitoyl-2-oleoyl-sn-glycero-3phosphocholine (POPC); B: 1-palmitoyl-2-oleoyl-sn-glycero-3-phospho-(1'-rac-glycerol) sodium salt, (POPG); C: 1-palmitoyl-2-oleoyl-sn-glycero-3-phosphoethanolamine (POPE); D: 1-palmitoyl-2-oleoylsn-glycero-3-phospho-L-serine sodium salt (POPS). 
in chloroform from Avanti Polar Lipids (Alabaster, Alabama, USA) and used as supplied. Their structures are given in Figure $\mathbf{3 . 1}$ (p. 44).

\subsubsection{Dyes}

\section{Membrane dyes}

The structures for all employed membrane dyes are given in Figure 3.2.

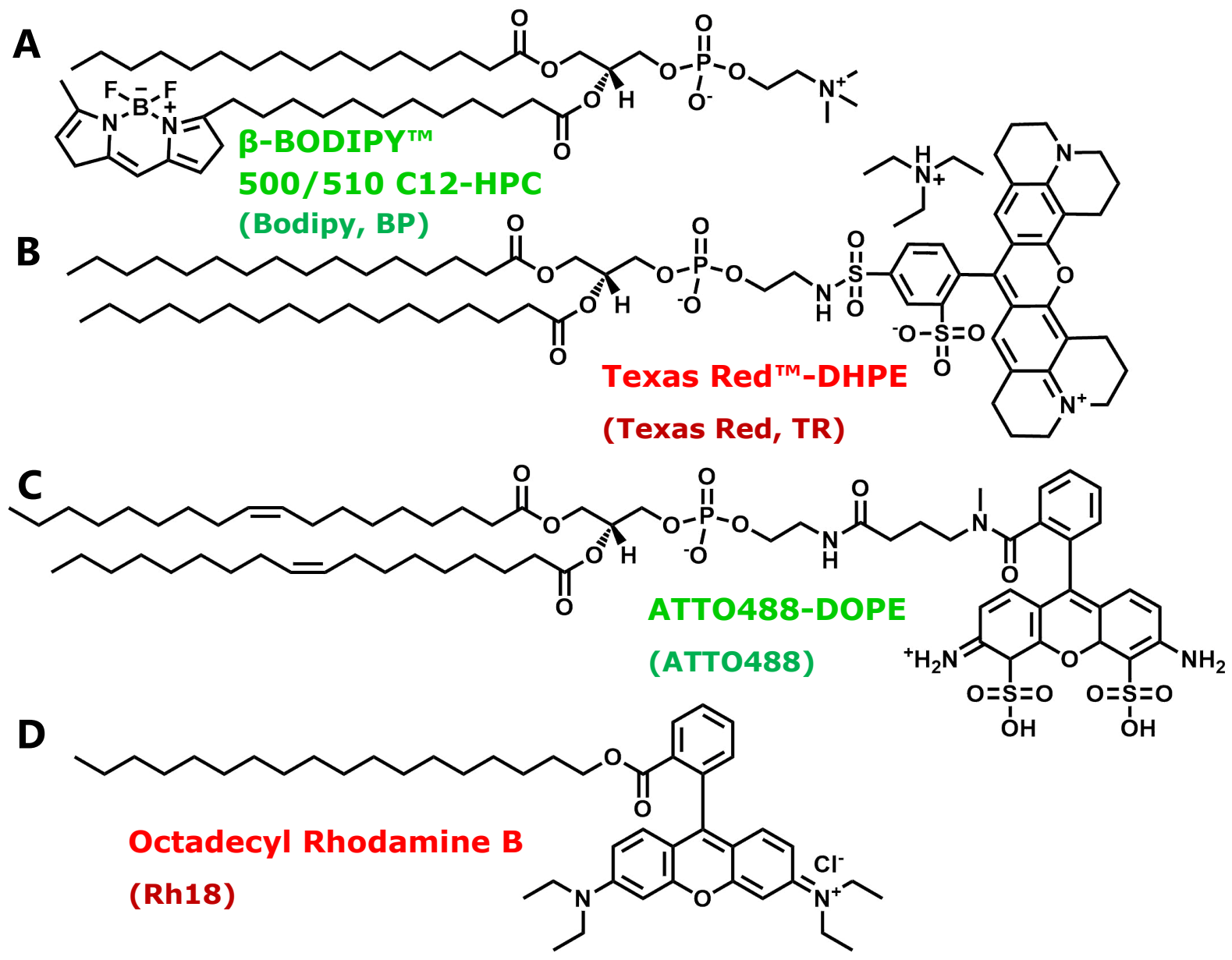

Figure 3.2: Chemical structures of membrane dyes used in this work. Manufacturer names for each dye are indicated, as well as colloquial abbreviations for these dyes used in this work. A: 2-(4,4-Difluoro-5Methyl-4-Bora-3a,4a-Diaza-s-Indacene-3-Dodecanoyl)-1-Hexadecanoyl-sn-Glycero-3-Phosphocholine $\left(\beta-B O D I P Y^{\top M}\right.$ 500/510 C12-HPC, Bodipy, BP); B: Texas Red ${ }^{\mathrm{TM}}$ 1,2-Dihexadecanoyl-sn-Glycero-3Phosphoethanolamine, Triethylammonium Salt (Texas Red ${ }^{\mathrm{TM}}$-DHPE, TR); C: 1,2-Dioleoyl-sn-glycero-3phosphoethanolamine labeled with ATTO488 (ATTO488-DOPE, ATTO488); D: 3,6-bis(diethylamino)-9(2-octadecyloxy)carbonyl)phenyl chloride (Rh18).

The lipid-tail fluorophore $\beta$-BODIPY'M 500/510 C12-HPC (2-(4,4-Difluoro-5Methyl-4-Bora-3a,4a-Diaza-s-Indacene-3-Dodecanoyl)-1-Hexadecanoyl-snGlycero-3-Phosphocholine, further denoted as Bodipy or BP) was obtained from ThermoFisher Scientific, Schwerte, Germany. It was dissolved to $0.1 \mathrm{mg} / \mathrm{mL}$ in HPLC-grade ethanol-stabilised chloroform and stored in a glass vial at $-24{ }^{\circ} \mathrm{C}$. 
The lipid headgroup fluorophore Texas $\operatorname{Red}^{\mathrm{TM}}$-DHPE (Texas Red ${ }^{\mathrm{TM}}$ 1,2Dihexadecanoyl-sn-Glycero-3-Phosphoethanolamine, Triethylammonium Salt) was obtained from ThermoFisher Scientific (Schwerte, Germany). It was dissolved to a $1 \mathrm{mg} / \mathrm{mL}$ solution in HPLC-grade chloroform and stored in a glass vial at $-24{ }^{\circ} \mathrm{C}$.

The lipid headgroup fluorophore ATTO488-DOPE (1,2-Dioleoyl-sn-glycero-3phosphoethanolamine labelled with ATTO488) was obtained from ATTO-TEC (Siegen, Germany) and dissolved to a $1 \mathrm{mg} / \mathrm{mL}$ solution in HPLC-grade chloroform and stored in a glass vial at $-24^{\circ} \mathrm{C}$.

The membrane staining agent Octadecyl Rhodamine B Chloride (Rh18, 3,6bis(diethylamino)-9-(2-octadecyloxy)carbonyl)phenyl chloride) was bought from ThermoFisher Scientific (Schwerte, Germany). It was dissolved to $10 \mathrm{mg} / \mathrm{mL}$ in DMSO and stored at $-24^{\circ} \mathrm{C}$.

\section{Water-soluble dye for leakage assays}

The structure of the water-soluble dye Sulforhodamine B for leakage assays is given in Figure 3.3. Sulforhodamine B sodium salt (sodium;4-(3-(diethylamino)6-diethylazaniumylidene-2,7-dimethylxanthen-9-yl)benzene-1,3-disulfonate) was bought from Merck (Darmstadt, Germany). A $50 \mathrm{mM}$ stock solution was prepared in measuring buffer, which was stored at $4^{\circ} \mathrm{C}$ before use.

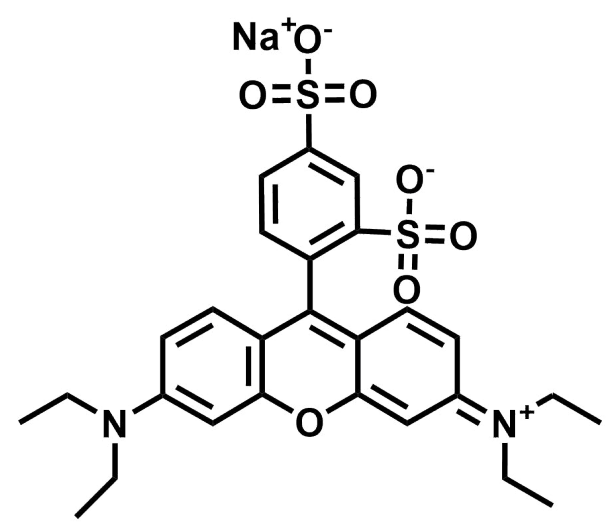

Figure 3.3: Chemical structure of Sulforhodamine B sodium salt.

\subsubsection{Arginine derivatives and peptides}

1-propyl guanidine (N-propylguanidine, Rside) was bought from SelectLab Chemicals (Münster, Germany). All peptides were ordered from Bachem (Heidelberg, Germany). Diarginine ( $\mathrm{H}$-Arg-Arg- $\mathrm{OH}, \mathrm{R}_{2}$ ) and tetra-arginine (H-ArgArg-Arg-Arg-OH, $\mathrm{R}_{4}$ ) were bought as an acetate salt. Hexa-arginine (H-Arg-Arg- 
Arg-Arg-Arg-Arg-OH， R6) and hexalysine (H-Lys-Lys-Lys-Lys-Lys-Lys-OH, $\mathrm{K}_{6}$, obtained via custom order) were obtained as a trifluoroacetate salt. Each Rderivative was dissolved to a $1 \mathrm{M}$ or $0.5 \mathrm{M}$ solution in measuring buffer and $100 \mathrm{mM}$ and $200 \mathrm{mM}$ stock solutions were diluted from this solution with the same buffer. All R-derivative stock solutions were stored at $-24^{\circ} \mathrm{C}$.

\subsubsection{Solid substrates}

\section{Silicon wafers}

Silicon wafers were obtained from Active Business Company GmbH (Munich, Germany). For atomic force microscopy and fluorescence microscopy, P-type, boron-doped silicon wafers of $725 \mu \mathrm{m}$ thickness with a $100 \mathrm{~nm} \mathrm{SiO}$-layer and a resistivity of 1-50 $\Omega \mathrm{cm}$ were used. Directly before use, they were cut to ca. $7 \times 22 \mathrm{~mm}$, thoroughly rinsed with water and ethanol p.a., and dried under nitrogen stream. They were treated with oxygen plasma in a Diener Zepto plasma cleaner (Diener Electronic, Ebhausen, Germany) as follows: 15 minutes of oxygen flow at 25 mbar, followed by a plasma process of 5.5 minutes at 25 mbar and $60 \%$ power. They were used immediately after this (see below).

For reflectometric interference spectroscopy, P-type, boron-doped silicon wafers of $525 \mu \mathrm{m}$ thickness with a $5000 \mathrm{~nm} \mathrm{S_{2 }}$-layer and a resistivity of 5-10 $\Omega \mathrm{cm}$ were used. They were cut to ca. $6 \times 19.5 \mathrm{~mm}$ and cleaned in a basic piranha solution (5:1:1 v/v/ $\mathrm{v} \mathrm{H}_{2} \mathrm{O}: 25 \% \mathrm{NH}_{4} \mathrm{OH}: 30 \% \mathrm{H}_{2} \mathrm{O}_{2}$ ) at $70{ }^{\circ} \mathrm{C}$ for 20 minutes, thoroughly rinsed with water and stored in ultrapure water before use. Before use, they were thoroughly rinsed with water and ethanol p.a. and dried under $\mathrm{N}_{2}$ stream. Subsequently, they were treated in a Diener Zepto plasma cleaner as follows: 5.5 minutes of oxygen flow at 0.20 mbar, followed by a $30 \mathrm{~s}$ plasma process at $60 \%$ power. Wafers were hydrated within five minutes after this (see below).

\section{Polydimethylsiloxane (PDMS)}

Polydimethylsiloxane solid supports were prepared by Jörn Dietz, according to a procedure as described in his Master thesis. For the preparation of PDMS support for the production of non-continuous membrane patches, a two-component Sylgard 184 kit from Dow Corning (West Midland, USA) was used. The monomer and curing agent were mixed in a $10: 1 \mathrm{v} / \mathrm{v}$ ratio and stirred with a plastic stirrer. 
Air bubbles were removed by 15 minutes exposure to vacuum at room temperature. Ca. $200 \mu \mathrm{L}$ (1 drop) of the resulting mixture were poured onto a $35 \mathrm{~mm}$ Ibidi $\mu$-dish (Uncoated \#1.5 polymer coverslip bottom, hydrophobic, sterilised, Ibidi, Gräfelfing, Germany). A thin layer of PDMS was spin-coated on the bottom of the dish at $950 \mathrm{rpm}(10 \mathrm{rpm} / \mathrm{s}$ acceleration and deceleration rate) for $50 \mathrm{~s}$ on a SCS G3P-8 spincoater (Specialty Coating Systems, Indianapolis, USA). The resulting ca. $100 \mu \mathrm{m}$-thick PDMS film was further hardened for 45 minutes at $70{ }^{\circ} \mathrm{C}$. Afterwards, prepared dishes with PDMS were covered and stored at room temperature.

\subsection{Preparation of lipid vesicles}

\subsubsection{Preparation of lipid films in desired compositions}

Vesicles were created from dried lipid films by hydration. For the creation of these lipid films, glass tubes were thoroughly rinsed with water, ethanol p.a. and HPLCgrade chloroform before use. Lipids and desired dye stock solutions were combined in a glass tube in the desired ratio for a $0.5 \mathrm{mg}$ lipid film for microscopy and reflectometric interference spectroscopy (RIfS) or a $2 \mathrm{mg}$ lipid film for leakage assays (overview in Table 3.2). After all necessary stock solutions were added together, the walls of the glass tube were rinsed with $150 \mu \mathrm{L}$ of chloroform, to gather all lipids at the bottom of the tube. Solvents were removed in a water bath

Table 3.2: Lipid compositions of lipid films used in this work. Dye = Bodipy, Texas Red-DHPE, or ATTO488-DOPE.

\begin{tabular}{ccc} 
Weight \& experiment & Lipid composition & Molar ratio \\
\hline \multirow{2}{*}{$\mathbf{. 5} \mathbf{~ m g}$} & POPC:Dye & $99: 1$ \\
Microscopy \& RIfS & POPC:POPE:Dye & $49.5: 49.5: 1$ \\
& POPC:POPG:Dye & $49.5: 49.5: 1$ \\
& POPC:POPS:Dye & $49.5: 49.5: 1$ \\
& POPC:POPG & $1: 1$ \\
& POPC:POPS & $1: 1$ \\
\hline \multirow{2}{*}{$\mathbf{~ m g}$} & POPC:POPG & $1: 1$ \\
Leakage assays & POPC:POPS & $1: 1$ \\
& POPC:POPS & $1: 1$
\end{tabular}


set to $35^{\circ} \mathrm{C}$ under nitrogen stream and subsequent exposure to vacuum at $55^{\circ} \mathrm{C}$ overnight. Lipid films were covered and stored at $4{ }^{\circ} \mathrm{C}$ for two weeks at most.

\subsubsection{Preparation of vesicles}

Lipid films for microscopy and RIfS were hydrated with $600 \mu \mathrm{L}$ of measuring buffer and left to swell 10 minutes (at room temperature for POPG- or POPS-containing lipid films, in a $55^{\circ} \mathrm{C}$ water bath for other films). Subsequently they were suspended in the glass tube to yield multilamellar polydisperse vesicles, and transferred to a $1.5 \mathrm{~mL}$ microcentrifuge tube. Small unilamellar vesicles (SUVs) were obtained by sonication for $30 \mathrm{~min}$ in a Bandelin Sonopuls HD2070 sonifier equipped with a BR30 beaker resonator and an $\mathrm{EH} 3$ reaction cup holder (Bandelin Electronic, Berlin, Germany), at $65 \%$ power and a $4 \times 10 \%$ pulsed cycle. Vesicles composed of mainly POPC or a mixture of POPC and POPE were additionally pressed over a $0.45 \mu \mathrm{m}$ PTFE syringe filter (diameter $13 \mathrm{~mm}$, VWR International, Hanover, Germany).

Lipid films for leakage assays were hydrated in $400 \mu \mathrm{L}$ of desired buffer (either phosphate measuring buffer containing $50 \mathrm{mM}$ sulforhodamine $B$ or Hepes buffer containing $20 \mathrm{mM}$ 5(6)-carboxyfluorescein as described under Buffers on $p$. 43) and left to swell as described above. After suspension, they were extruded 31 times over a $100 \mathrm{~nm}$ polycarbonate filter (Avestin Europe, Mannheim, Germany) using an Avestin LiposoFast mini extruder system to yield LUVs. To exchange the buffer outside the vesicles for a buffer without dye, vesicles were added to an Illustra ${ }^{\mathrm{TM}}$ NAP $^{\mathrm{TM}}$ disposable column prepacked with Sephadex ${ }^{\mathrm{TM}}$ G-25 DNA grade (GE Healthcare, Little Chalfont, Buckinghamshire, United Kingdom) which was preequilibrated with $20 \mathrm{~mL}$ of measuring buffer. Subsequently, $2 \mathrm{~mL}$ of measuring buffer was added slowly. Next, $4 \times 500 \mu \mathrm{L}$ of measuring buffer were added, and these fractions were collected. The two fractions that visually contained most of the dye were combined and stored for a leakage assay. The procedure yielded a ca. $2.5 \times$ dilution of the vesicles, which was used to calculate the estimated peptide-to-lipid ratios. 


\subsection{Leakage assays with $\mathrm{R}_{6}$}

Leakage assays with sulforhodamine B were performed on a HORIBA Fluoromax-4 spectrofluorometer controlled by FluorEssence ${ }^{\mathrm{TM}}$ software version 3.8 (HORIBA Europe, Hanover, Germany). Samples were prepared as $1 \mathrm{~mL}$ containing 0.01 $\mathrm{mg} / \mathrm{mL}$ lipids in measuring buffer in disposable $1.5 \mathrm{~mL}$ PMMA cuvettes (Brand, Wertheim, Germany). Sulforhodamine B fluorescence intensity was monitored over time at $565 \mathrm{~nm}$ excitation and $585 \mathrm{~nm}$ emission wavelengths (excitation slit $1 \mathrm{~nm}$, emission slit $2 \mathrm{~nm}$, increment $0.1 \mathrm{~s}$, integration time $0.1 \mathrm{~s}$ ). The solution was stirred continuously by a $3 \times 3 \mathrm{~mm}$ micro stirring bar (VWR International, Hanover, Germany). $R_{6}$ was added at ca. every $500 \mathrm{~s}$ in increasing concentrations (from stock solutions that had 100x the desired concentration in measuring buffer, resulting in additions of ca. $10 \mu \mathrm{L}$ ). $100 \%$ leakage was determined by addition $20 \mu \mathrm{L}$ of 10 -fold diluted Triton X-100 in measuring buffer. Sulforhodamine B was normalised to $0 \%$ leakage for its fluorescence intensity at the start of the assay and to $100 \%$ for its intensity after addition of Triton X-100 solution. Data were corrected for linear drift by subtracting a line fitted to the fluorescence data before $\mathrm{R}_{6}$ addition.

\subsection{Solid-supported lipid bilayers for microscopy}

\subsubsection{Silicondioxide-supported membranes}

Silicon wafers with a $100 \mathrm{~nm} \mathrm{SiO}$ layer were inserted into Teflon sample chambers immediately after plasma cleaner treatment (see Solid substrates p. 47) and hydrated with $350 \mu \mathrm{L}$ of spreading buffer (pictures of chambers in Figure 3.4). 
$250 \mu \mathrm{L}$ of vesicle solution (see Preparation of vesicles p. 49) was added and a membrane was left to form for two hours at room temperature. $400 \mu \mathrm{L}$ of measuring buffer was added, and remaining vesicles were rinsed off with $6 \times 1 \mathrm{~mL}$ of measuring buffer. $600 \mu \mathrm{L}$ of measuring buffer was added again to yield a sample volume of $1600 \mu \mathrm{L}$.
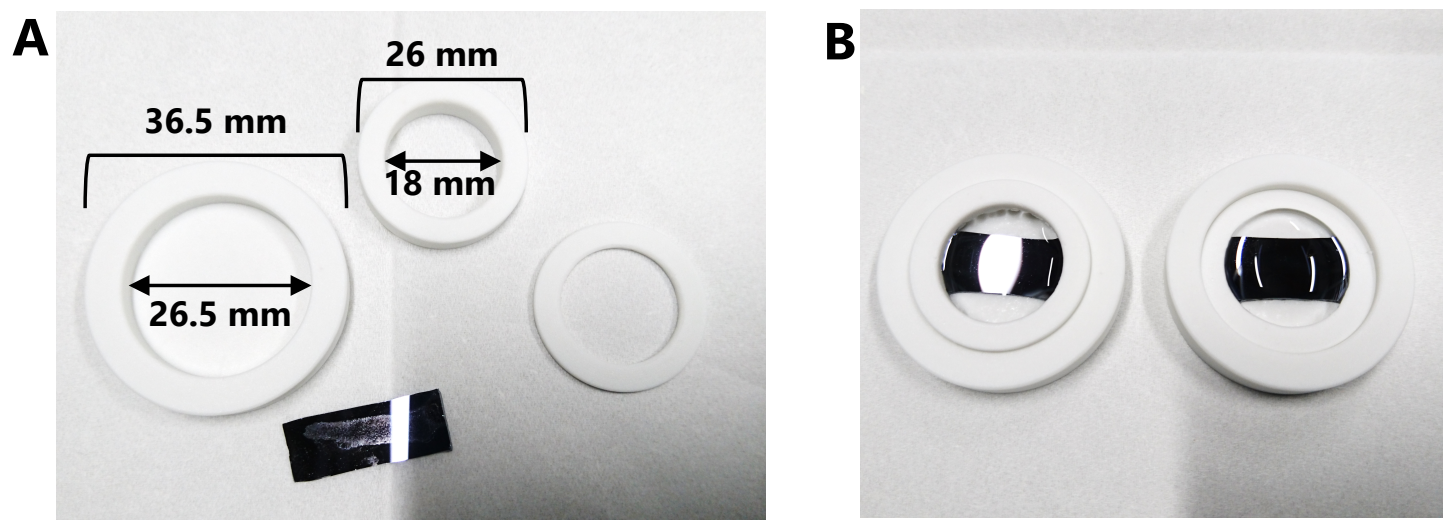

Figure 3.4: Pictures of Teflon sample chambers for solid-supported lipid bilayers. Chambers consist of a circular base ( $36.5 \mathrm{~mm}$ outer diameter) and a tightly fitting pressure ring (outer diameter $26 \mathrm{~mm}$ ) A: Chamber base, pressure ring and silicon wafer disassembled, with measurements for the sample chamber parts indicated in the picture; B: Assembled and hydrated chambers.

\subsubsection{PDMS-supported membrane patches}

PDMS was prepared in an Ibidi polymer coverslip bottom dish as described in Section 3.1.5 Polydimethylsiloxane (PDMS) ( $p$. 47). It was covered by an Aquamarijn silicon microsieve chip with $5 \mu \mathrm{m}$ pore size in the $\mathrm{SiO}_{2}$ porous area (rectangular, Aquamarijn, Zutphen, Netherlands). This facilitated oxidation of defined $5 \mu \mathrm{m}$ patches of PDMS by oxygen plasma (30 s at 20 mbar and $20 \%$ power, Diener Zepto plasma cleaner, Diener Electronic, Ebhausen, Germany). Subsequently, the microsieve chip was removed and vesicle spreading was performed as described above for silicon wafers.

\subsubsection{Addition of R-derivatives}

R-derivatives or peptides were added from a concentrated stock solution (typically $100 \times$ desired sample concentration) and mixed upon adding. The volume added was in the order of $10 \mu \mathrm{L}$ for $\mathrm{R}$-peptides and $100 \mu \mathrm{L}$ for $\mathrm{R}_{\text {side }}$ or $\mathrm{R}_{2}$. 


\subsubsection{Addition of vesicles}

To explore the insertion of lipid material from solution into the solid-supported lipid membrane under influence of $R_{6}$, a solution of vesicles with a different dye than the solid-supported membrane was prepared according to the procedure described in 3.2 Preparation of lipid vesicles (p. $48 \mathrm{ff}$.). $8 \mu \mathrm{L}$ of vesicle solution was added to the sample, to yield an equivalent concentration of vesicles that is expected to be left after membrane preparation (ca. $0.004 \mathrm{mg} / \mathrm{mL}$ ). Vesicles were added before addition of $\mathrm{R}_{6}$.

\subsubsection{In situ staining of membrane stacks with Rh18}

A membrane (POPC:POPG 1:1) without dye was prepared as described above. $\mathrm{R}_{6}$ was added to the membrane to a concentration of $1 \mu \mathrm{M}$ and left to incubate for 1 hour. Rh18 was diluted $1000 \times$ in measuring buffer. $160 \mu \mathrm{L}$ of this solution were added to the membrane and left to incubate for ca. $5 \mathrm{~min} .500 \mu \mathrm{L}$ of liquid was removed from the sample and the membrane was rinsed with $4 \times 1 \mathrm{~mL}$ of measuring buffer containing $1 \mu \mathrm{M} \mathrm{R}_{6}$.

\subsection{Atomic force microscopy}

\subsubsection{Breakthrough force spectroscopy}

Force spectroscopy experiments were carried out on a JPK NanoWizard 3 or 4 (JPK Instruments, Berlin, Germany). Bruker MLCT silicon nitride microlevers with silicon nitride tips were used in all AFM experiments (tip radius 20-60 nm, Bruker France, Wissembourg Cédex, France) Triangular probe D or E on a chip was always used, with determined spring constants of ca. 0.060 or $0.200 \mathrm{~N} / \mathrm{m}$, respectively. Spring constants were calibrated by using the thermal noise method combined with determination of the ratio of PSD-voltage to piezo movement as detailed in Methods Background $[115,116]$.

All breakthrough force experiments were performed with $1 \mu \mathrm{m} / \mathrm{s}$ approach and retract velocity. At least 2000 breakthrough force curves were collected of the solid-supported lipid bilayer without the R-derivative. Maximum force and retract 
distance were chosen according to the sample. Then, arginine derivative was added to the desired concentration (see above), and at least 2000 breakthrough force curves were collected again.

\subsubsection{AFM imaging}

QI Mode ${ }^{T M}$ height images correlated with fluorescence microscopy images as shown in Figure 5.1 p. 92 and Appendix Figure A 10.1 p. XXXIII were recorded on a JPK NanoWizard $® 4$ combined with a BioMAT ${ }^{\mathrm{TM}}$ workstation (JPK Instruments, Berlin, Germany). The microscopy table was mounted on an Olympus BX53 upright microscope equipped with an Olympus 60× objective (LUMPFLN60xW, Olympus, Hamburg, Germany) and an Andor Zyla sCMOS camera (Oxford Instruments Andor, Belfast, United Kingdom). The force setpoint was set to ca. $0.1 \mathrm{nN}$ and the retraction distance was set to ca. $60 \mathrm{~nm}$ (exact values differed per image).

\subsubsection{Data processing}

\section{Images}

Height images were processed using JPKSPM data processing software (version 6.0.40, JPK Instruments, Berlin, Germany) as well as Gwyddion version $2.54[142]$.

\section{Breakthrough force data}

Basic processing of the force curves was performed using JPKSPM data processing software (version 6.0.40 JPK Instruments, Berlin, Germany) to obtain baselinecorrected force-distance curves with determined contact point (as single files). Automatic analysis of breakthrough events was performed using a Matlab script written by Dr. Ingo Mey (Institute of Organic and Biomolecular Chemistry, University of Göttingen). Before analysis, smoothing of the force curve data by a moving mean filter was performed (Illustration in Figure 3.2). The script then analyses the force curve where the force is above a pre-set threshold, and then applies a threshold to the first derivative. Thus, the breakthrough event (with a much less steep slope than the rest of the curve in the contact range) could be filtered out as points in a plateau determined as the breakthrough event (indicated as red $x$-es in Figure 3.2). The breakthrough force was defined as the average force of the points in the breakthrough event. The breakthrough distance was 
defined as the difference between the minimum and the maximum of the $x$-values of the points in the breakthrough event.

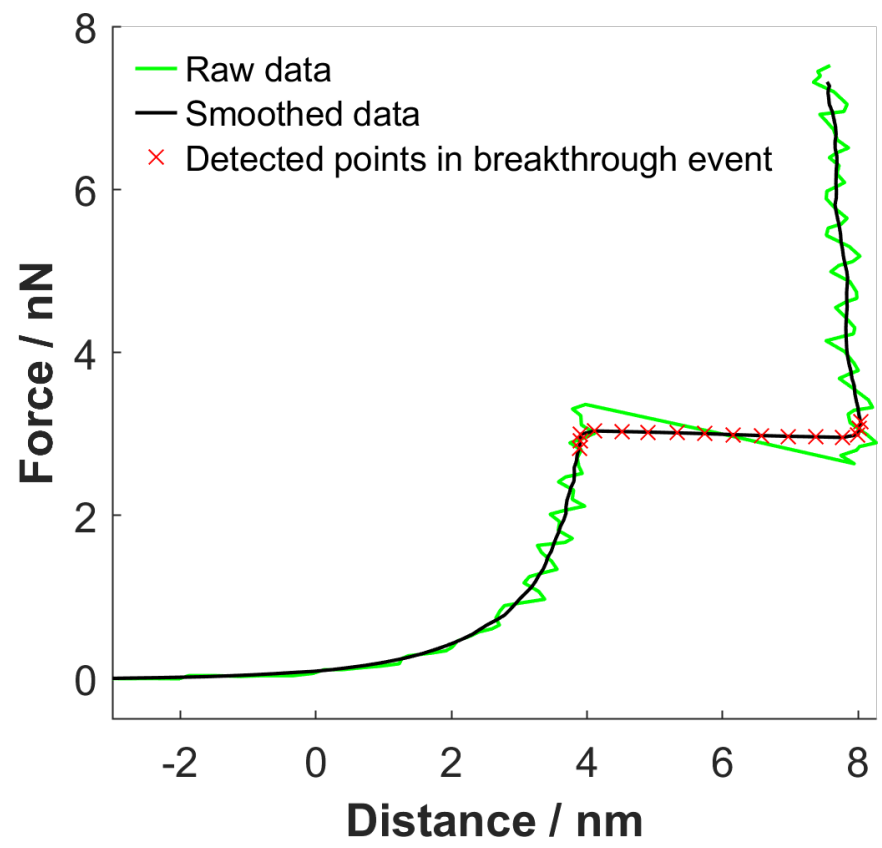

Figure 3.5: Illustration of a breakthrough event in an indentation force curve as processed by the script written by Dr. Ingo Mey.

At least three experiments were performed for each combination of lipid mixture and R-derivative. It was experienced that the median force detected differed per experiment and per cantilever used. This was attributed to possible differences in tip radius of individual cantilevers, which likely influences the breakthrough force distribution and penetration depth before the event occurs (see Methods Background p. 34). Therefore, experimental results of breakthrough force and breakthrough distance were normalised for the median of the distribution for the pure lipids measured with the same cantilever. The median provides a more reliable quantity than the mean if the distribution is asymmetric. In this way, experimental results could be compiled to combined datasets. 


\subsection{Reflectometric Interference Spectroscopy (RIfS)}

Reflectometric interference spectroscopy was performed using a tungsten halogen light source (HL-2000-FHSA Ocean Optics, Ostfildern, Germany) combined with an Ocean Optics flame miniature spectrometer (FLAME-S-UV-VIS-ES, Ocean Optics, Ostfildern, Germany). A reflection spectrum between 500 and $700 \mathrm{~nm}$ wavelengths was recorded every $2 \mathrm{~s}$ using SpectraSuite software (Ocean Optics, Ostfildern, Germany). Polished aluminium was used as the reference for full reflection of the illuminating light. Calculation of optical thickness OT was performed live during the experiment using a Matlab-based graphical user interface originally developed by Dr. Milena Stephan and Dr. Ingo Mey, revised by Dr. Ingo Mey. To summarize, equation (2.9) was fitted to the first spectrum recorded to obtain the optical thickness and refractive index of the $\mathrm{SiO}_{2}$-layer

A

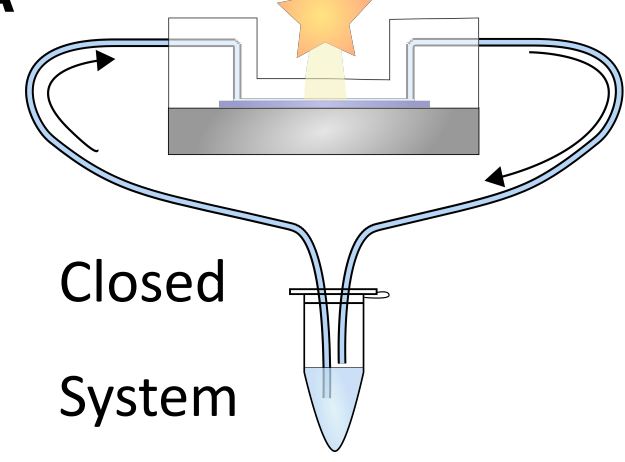

B

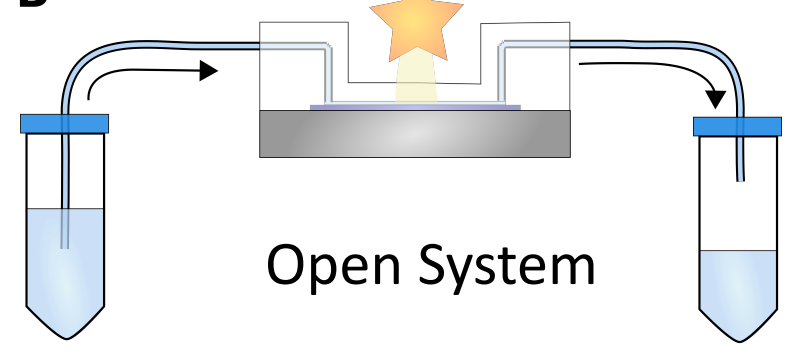

Figure 3.6: Schematic illustration of a closed and an open system for the flow cells in RIfS experiments. The yellow star signifies the light source and spectrometer. Arrows indicate flow direction. A: Closed system, inlet and outlet of flow connected to the same container; This was typically used to increase the concentration of R-peptide, by using a microcentrifuge tube with the desired amount of R-peptide stock solution. B: Open system, inlet typically a larger volume, outlet a waste container. This was typically used to rinse the bilayer after its formation.

in spreading buffer by inserting a guess value for its thickness. The refractive indices of water and silicon (1.33 and 3.87, respectively) were inserted in to the equation. From there, the change in optical thickness was calculated while assuming by approximation that the $\mathrm{SiO}_{2}$-layer changed its thickness. 
For the experiment, silicon wafers with a $5000 \mathrm{~nm} \mathrm{SiO}$ layer (as described under Solid-supported lipid bilayers for microscopy p. 50) were inserted into custom made flow cells consisting of an aluminium base with an acrylic glass cover as used by Stephan et al. [143], except that the aluminium base was flat instead of indented. Flow rate in all experiments was $0.43 \mathrm{~mL} / \mathrm{min}$. All buffers were newly degassed before a RIfS experiment. The system was hydrated in ultrapure water, which was exchanged for spreading buffer when the system was stable. To form the solid-supported lipid bilayer, $200 \mu \mathrm{L}$ of vesicle solution was added to the system, and it was left to circulate as a closed system (Figure 3.3 A) for 45 minutes.

For determination of dissociation constants of R-peptides, vesicles were rinsed out of the system by a $20 \mathrm{~min}$ flow in an open system with measuring buffer (Figure 3.3 B). Then, R-peptide was added in increasing amounts in a closed system fashion.

For the RIfS experiments where vesicles were still present, the membrane was rinsed in a closed system fashion with $1200 \mu \mathrm{L}$ of measuring buffer for 5 minutes. This was repeated four more times. Given that the system volume was ca. $600 \mu \mathrm{L}$, it was estimated that after this procedure, the system contained approximately the same amount of lipid vesicles as the solid-supported lipid bilayers used for microscopy experiments. $R_{6}$ was added in a closed system fashion and left to circulate until optical thickness was stable.

\subsubsection{Data analysis}

Data processing was performed with a self-written Matlab script. Data were corrected for linear drift by subtracting a line fitted through the data of the optical thickness without R-peptide present. Plots of R-peptide concentration $[R]$ against optical thickness change $(\triangle O T)$ were obtained by fitting a horizontal line through data at each concentration, after the $O T$ had stabilised. Dissociation constants were obtained by fitting the following Langmuir equation to data from at least two experiments[144]:

$$
\Delta O T=\frac{\Delta O T_{\max } \cdot[\mathrm{R}]}{K_{\mathrm{D}}+[\mathrm{R}]}
$$


Where $\Delta O T_{\max }$ is the optical thickness at saturation, $[R]$ is the R-peptide concentration, and $K_{\mathrm{D}}$ is the dissociation constant. Data recorded with $\mathrm{R}_{6}$ were additionally fitted to a Hill-Waud equation:

$$
\Delta O T=\frac{\Delta O T_{\max } \cdot[\mathrm{R}]^{m}}{K_{D}{ }^{m}+[\mathrm{R}]^{m}}
$$

Where $m$ is the Hill coefficient, a measure for the level of cooperativity of binding[145, 146]. Fitting was performed using a Matlab script written by Prof. Dr. Andreas Janshoff and Prof. Dr. Burkhard Geil, which employed the Levenberg-Marquardt algorithm[147].

\subsection{Fluorescence microscopy}

General fluorescence microscopy was performed on an Olympus BX51 or BX53 upright microscope equipped with an Olympus 60x objective (LUMPFLN60xW, Olympus, Hamburg, Germany) and an Andor Zyla sCMOS camera (Oxford Instruments Andor, Belfast, United Kingdom). Confocal laser scanning microscopy and fluorescence recovery after photobleaching (FRAP) experiments were performed on an Olympus FV 1200 (Olympus, Hamburg, Germany) confocal laser scanning microscope equipped with a $60 x$ water immersion objective (LUMFLN N 60×/1.10 NA, Olympus, Hamburg, Germany), a 488 nm laser (85-BDD-020-003, Melles Griot, Bensheim, Germany) and a 561 nm laser (85YCA-020-230, Melles Griot, Bensheim, Germany). Bodipy and ATTO488 were excited at $488 \mathrm{~nm}$ at $4 \%$ laser intensity as set in Olympus software. Texas Red and Rh18 were excited at $561 \mathrm{~nm}$ and 4\% laser intensity as set in Olympus software.

Recorded images were exported as Tiff files and analysed with open source FIJI software[148]. For stack area analysis under continuous illumination, images were recorded every two seconds. Stack were automatically detected using FIJI software[148] by applying a gaussian blur to the images, manual thresholding, and using the function 'Analyze Particles'. The obtained 'particle' data were exported to a text file and stack area data were sorted and analysed over time with a self-written Matlab script. 


\subsubsection{Fluorescence monitoring after R- derivative addition}

Fluorescence monitoring after R-derivative addition was performed on a confocal laser scanning microscope as described above. Images were recorded every 10 or 15 seconds, and the R-derivative was typically added and mixed in between the first two frames. For stack growth analysis after R-peptide addition, data were analysed as described above.

\subsubsection{Fluorescence recovery after photobleaching}

Fluorescence recovery after photobleaching (FRAP) experiments were executed with an automatically recorded time series. For determination of the diffusion constant, the membrane was bleached for 1 second at $100 \%$ laser power in "tornado" scanning mode (Olympus, Hamburg, Germany). For FRAP of membrane stacks, a stack was typically bleached $1.5-2 \mathrm{~s}$.

The diffusion coefficient was determined from FRAP series using a Matlab program employing circular averaging of images and Hankel transforms, as developed by Jönsson et al.[149]. The source code was edited to enable direct reading of a metadata file and easier saving of analysis results.

FRAP of membrane stacks was analysed by a method as developed by Savić et al.[91, 150], to obtain an objective measure for the recovery rate when the diffusion constant is not a useful measure. This method is illustrated in Figure 3.7:
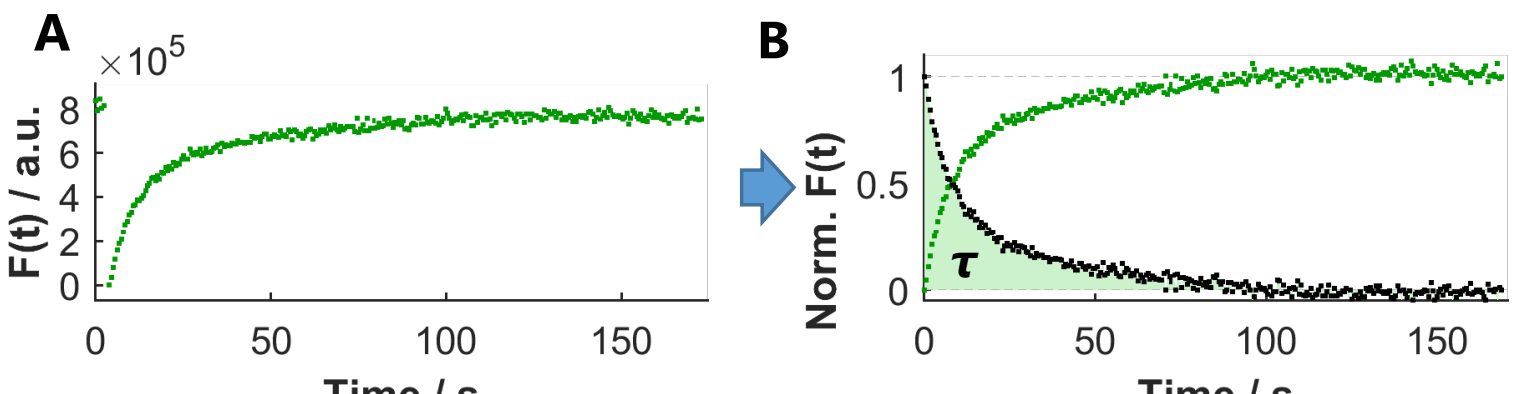

Figure 3.7 Illustration of the derivation of the mean time constant $\tau$ from integrated fluorescence intensity data from a FRAP experiment; A: An example dataset of the integrated fluorescence intensity after a FRAP experiment, corrected for bleaching and set to 0 for the fluorescence after bleaching; $\mathbf{B}$ : Illustration of the normalisation and integration performed to obtain mean time constant $\tau$ : fluorescence data is further normalised to 1 after fluorescence recovery (green dots), and these data are subtracted from 1 to lead to an inverse recovery curve (black dots). Mean time constant $\tau$ is obtained by integrating the flipped data over time (green shaded area). 
Integrated fluorescence intensity data $F(\mathrm{t})$ are normalised to 0 for the value immediately after bleaching and corrected for continuous bleaching. Subsequently, the recovery portion of the data is normalised to 1 for the maximum fluorescence of recovery (resulting in the green dataset in Figure 3.7 B). This normalised dataset is then subtracted from 1 (resulting in the black dataset in Figure 3.7 B) and integrated over time. The result of this integral is mean time constant $T$. 



\section{EFFECTS OF ARGININE}

\section{DERIVATIVES ON}

DIFFERENT LIPIDS

In this chapter, experiments probing the effects of arginine derivatives and oligopeptides on different lipid compositions will be presented. It will commence with results from Molecular Dynamics simulations of pore formation, which were performed by Dr. Neha Awasthi. The second subchapter will present breakthrough force spectroscopy outcomes, as this was a direct attempt to experimentally verify simulation results. Then thirdly, influence on membrane integrity was investigated in a different way, by means of leakage assays. Afterwards, effects on membrane fluidity were explored, by fluorescence recovery after photobleaching (FRAP) experiments. Lastly, visual changes to membranes, as investigated by fluorescence microscopy, will be presented. The chapter will be concluded with a discussion of the findings, as a bridge to the Chapter 5 ( $p .91 \mathrm{ff}$.) with further characterization results. 


\subsection{Simulations of pore formation}

Figure 4.1 presents the results of MD simulations directly related to this work. As mentioned before, simulations were performed by Dr. Neha Awasthi as part of a collaboration with the group of Prof. Dr. Jochen Hub (Saarland University). Figure 4.1 and its caption were adapted from images by Dr. Neha, with permission.

Pore formation was simulated in three different lipid bilayers, composed of either POPC, POPG or POPE. This was performed in the presence of three different arginine derivatives, further denoted as R-derivatives and individually referred to with the following abbreviations: $R_{2}$, a charged arginine dipeptide, with all side chains as well as the $\mathrm{N}$-terminus protonated and the $\mathrm{C}$-terminus deprotonated; $\mathrm{R}_{2}$ cap, an arginine dipeptide with neutral $\mathrm{N}$ - and $\mathrm{C}$-terminus to mimic a capped version of the peptide; and $\mathrm{R}_{\text {side, }}$ or actually 1 -propylguanidine, as a mimic of the arginine sidechain.

Figure 4.1 $\mathbf{A}$ and $\mathbf{B}$ show that only pore formation in POPG membranes seems to be significantly influenced, and mostly by $R$-derivatives $R_{2}$ cap and $R_{\text {side. In }}$ Figure 4.1 A, the kinks in the PMFs for pore formation as a function of the number of water molecules in the transmembrane cylinder $\left(\mathrm{N}_{\mathrm{w}}\right)$ are displayed. They are lowered by $R_{2}{ }^{\text {cap }}$ and $R_{\text {side }}$ in POPG membranes. Interestingly, the PMF was increased in case of $R_{2}$, for both POPG and POPC. Furthermore, as the general PMF was lowered in POPG membranes, the kink also occurred at a smaller number of water molecules in the transmembrane cylinder. This kink was used as an estimate for the pore nucleation energy, and plotted for each lipid and peptide in Figure 4.1 B. The presence of R-derivatives only decreased this pore nucleation energy noticeably in POPG membranes, which indicates more likely pore formation.

Besides this, other structural membrane properties also changed most in POPG bilayers, as plotted in Figure 4.1 B. Especially Rside seemed to cause membrane thinning in POPG, as shown by the increase of the membrane thinning modulus (top plot) and also the evident decrease in membrane thickness (lower middle 
plot). Furthermore, aforementioned changes in POPG membranes correlate with an increase in area per lipid (bottom plot). All these effects seem to be cumulative, as 64 units of Rside show a more pronounced change than 32 units of $R_{\text {side. }}$

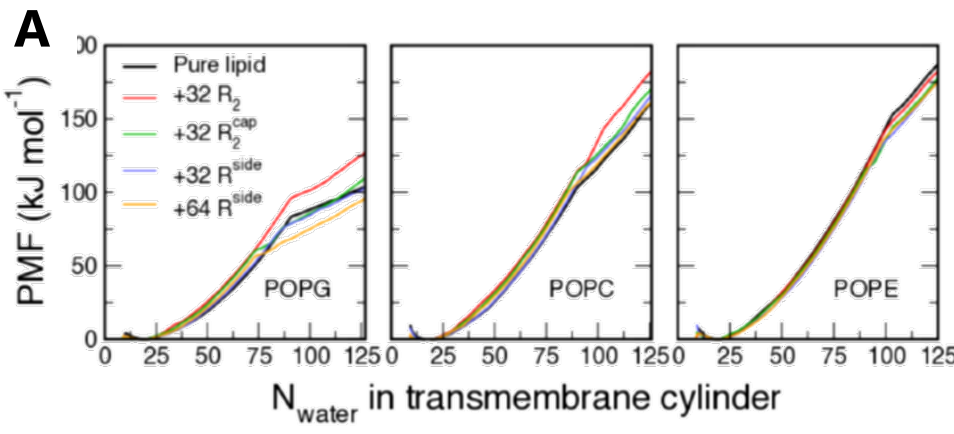

B

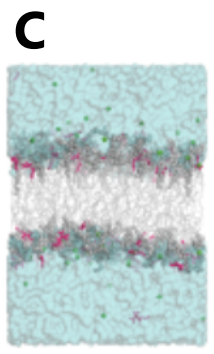

D
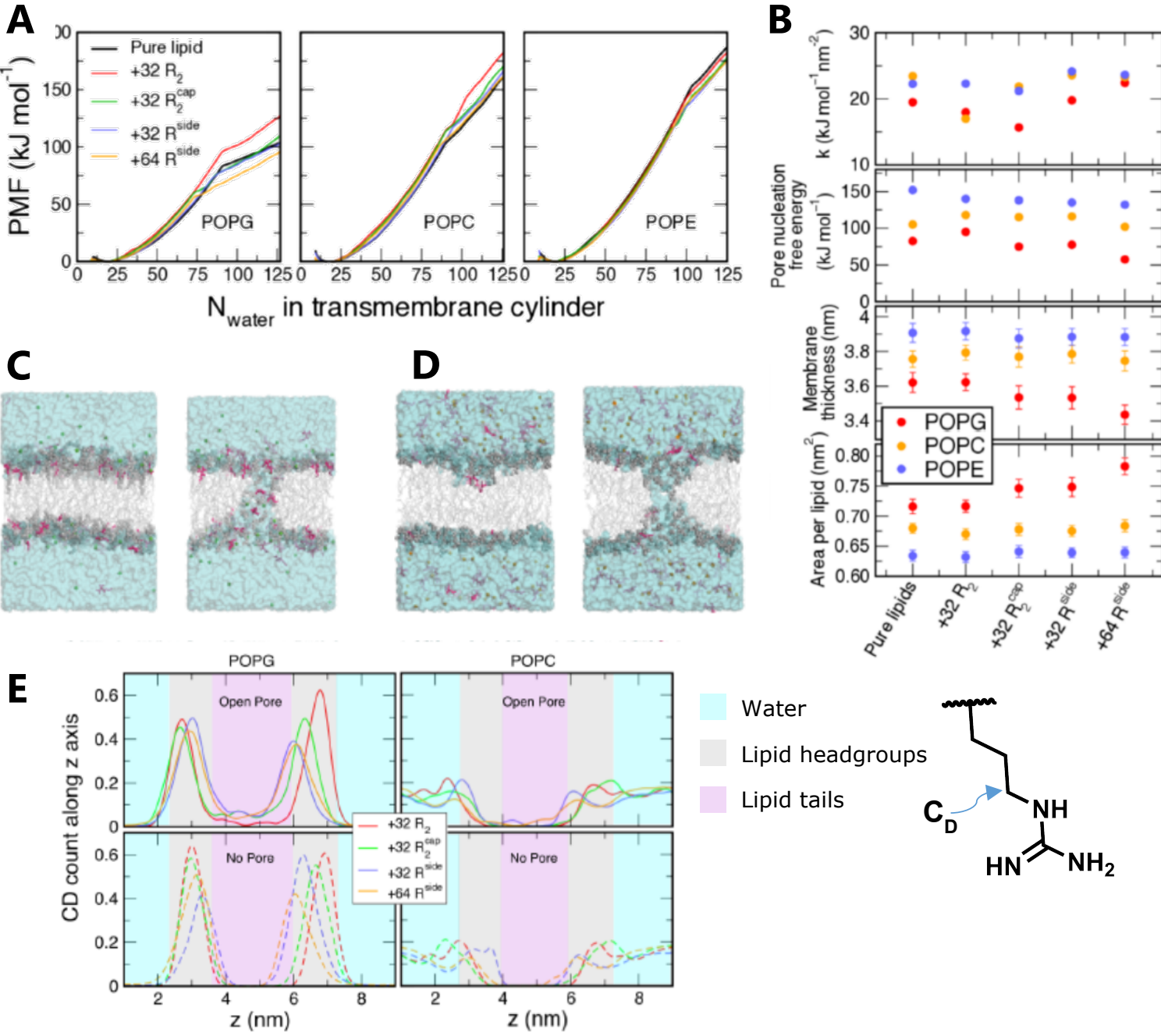

Figure 4.1: Molecular Dynamics simulation results for pore formation in bilayers composed of either POPG, POPE or POPC, combined with arginine derivatives $R_{2}, R_{2}$ cap , and $R_{\text {side. }} R_{\text {side }}$ was simulated as both 32 units per simulated box and 64 units per box. A: PMF of pore formation as a function of number of water molecules in the transmembrane cylinder, $N_{w}$ for the three different lipids and with each line colour representing one R-derivative. B: Structural properties of the three bilayers as a function of Rderivative present. Error bars indicate standard errors. From top to bottom: Membrane thinning modulus as obtained by fitting the quadratic region of the PMFs in $\mathbf{A}$; Pore nucleation energy as estimated from the kinks in PMFs in A, Membrane thickness and area per lipid, obtained by dividing the total surface of the simulated bilayer by the number of lipids. C \& $\mathbf{D}$ : initial $\left(\mathrm{N}_{\mathrm{w}}=20\right)$ and final $\left(\mathrm{N}_{\mathrm{w}}=120\right)$ snapshots of pore formation in the presence of $64 \mathrm{R}_{\text {side }}$ units, for POPG and POPC, respectively. E: A numerical representation of C \& $\mathbf{D}$ snapshots but then for all R-derivatives, 'No pore' corresponding to the initial snapshot and 'Open pore' corresponding to the final snapshot. The $x$-axis signifies distance $\mathrm{z}$ in $\mathrm{nm}$ perpendicular to the bilayer with colour coding for the aqueous phase, the headgroups and the lipid tails. The $y$-axis shows the normalized count of $C_{D}$-atoms of the R-derivatives. This makes the lines in the plot a representation for the relative abundancy of R-derivatives either in the aqueous phase, or in association with the bilayer. 
Figure 4.1 C-E illustrates the relationship between above-presented changes in parameters for POPG membranes and apparent binding behaviour of R-derivatives. When comparing the snapshots of POPG membranes (Figure 4.1 C) to POPC membranes (Figure 4.1 D), for POPC, $R_{\text {side }}$ is visually much less associated with the membranes and more present in the water matrix. This trend is further illustrated in a numerical fashion and for all R-derivatives in the comparative plots of Figure 4.1 E. Relative frequency of $C_{D}$ atoms (defined as the carbon atom closest to the guanidine moiety, as illustrated on the right) is shown along an axis perpendicularly crossing through the membrane. The water matrix, lipid headgroup and lipid tails are colour coded along this axis. These plots are therefore an illustration of where R-derivatives most likely accumulate. In case of POPG membranes, nearly all R-derivatives are located near the lipid headgroups and almost none are found in the water matrix. In case of POPC, R-derivatives seem to prefer the water matrix above any region in the membrane, although some association with the headgroups can still be observed.

These findings and their implications provided an incentive to investigate whether similar results could be confirmed experimentally. Results from corresponding in vitro experiments are presented in the rest of this chapter.

\subsection{Breakthrough force analysis}

In aforementioned pore formation simulations, membrane failure was induced, by forcing water molecules through a defined region. Breakthrough force spectroscopy also involves induction of failure of a thin molecular film, by forcing a cantilever tip through it (see p. 33)[124-127, 151].

Simulation conditions could not be replicated one on one, due to physical limitations of the materials. The lipids POPG and POPE were evaluated as a 1:1 mixture with POPC, due to their unlikelihood of forming a stable solid-supported planar membrane on their own. In case of POPE, this is due to its small headgroup, causing a cone shape, and hence a preference for negatively curved membrane geometries [152]. POPG, while being cylindrical in shape just like POPC[153], has a net negative charge, which was shown to lead to very soft membranes with many defects [154]. 
Three arginine derivatives were evaluated: $R_{2}, R_{\text {side }}$ and a hexa-arginine peptide, further referred to as $R_{6}$. Furthermore, experiments with lysine were performed. The motivation for this was that several studies implicate that lysine (henceforth called $\mathrm{K}$ ) is not as effective as arginine in a peptide, in model systems[8, 36] as well as functionality as a cell-penetrating peptide [18, 33, 37, 38].

As mentioned in Chapter 2.2.1 Breakthrough force spectroscopy (p. 33), due to differences in cantilever tip radius, repeats of the same experiment had to be normalised in order to be able to assess reproducibility and to compare them to other experiments. All individual experimental results are provided in Appendix 2-4 p. II-XIX with their absolute breakthrough force and breakthrough distance values.

To give an impression of typical values measured, exemplary results of absolute values of breakthrough force and breakthrough distance for pure lipids will be provided first. Then, comparative normalised breakthrough force results on lipids combined with different R-derivatives will be presented, followed by normalized results of breakthrough distances.

\subsubsection{Lipid mixtures without R-derivatives}

Figure 4.2 presents exemplary results from breakthrough force experiments for each lipid mixture, without R-derivatives present. Values obtained differed per experiment and cantilever used. Absolute values for breakthrough force and breakthrough distance of all individual experiments can be found in Appendix 2-4 p. II-XIX. In Figure 4.2 A, exemplary datasets of breakthrough forces for all pure lipid mixtures are shown. The detected forces in Figure 4.2 ranged from 2 to $4 \mathrm{nN}$. It has to be noted that for all lipid mixtures, median forces spread throughout this range and beyond ( $\sim 0.5-8 \mathrm{nN}$, see Appendix 2-4 p. II-XIX) have been obtained in individual experiments. Therefore, the visible differences in this plot are only illustrative of the general range of forces detected in most experiments. They are not to be interpreted as actual differences in mechanics between different membrane compositions.

Figure 4.2 B depicts exemplary absolute values of the breakthrough distance for all used lipid mixtures. No obvious differences between lipid mixtures were observed, only between individual experiments. In most experiments, the breakthrough distance was within the range of 3-5 nm (see also Appendix 2-4 p. $I I-X I X)$. 

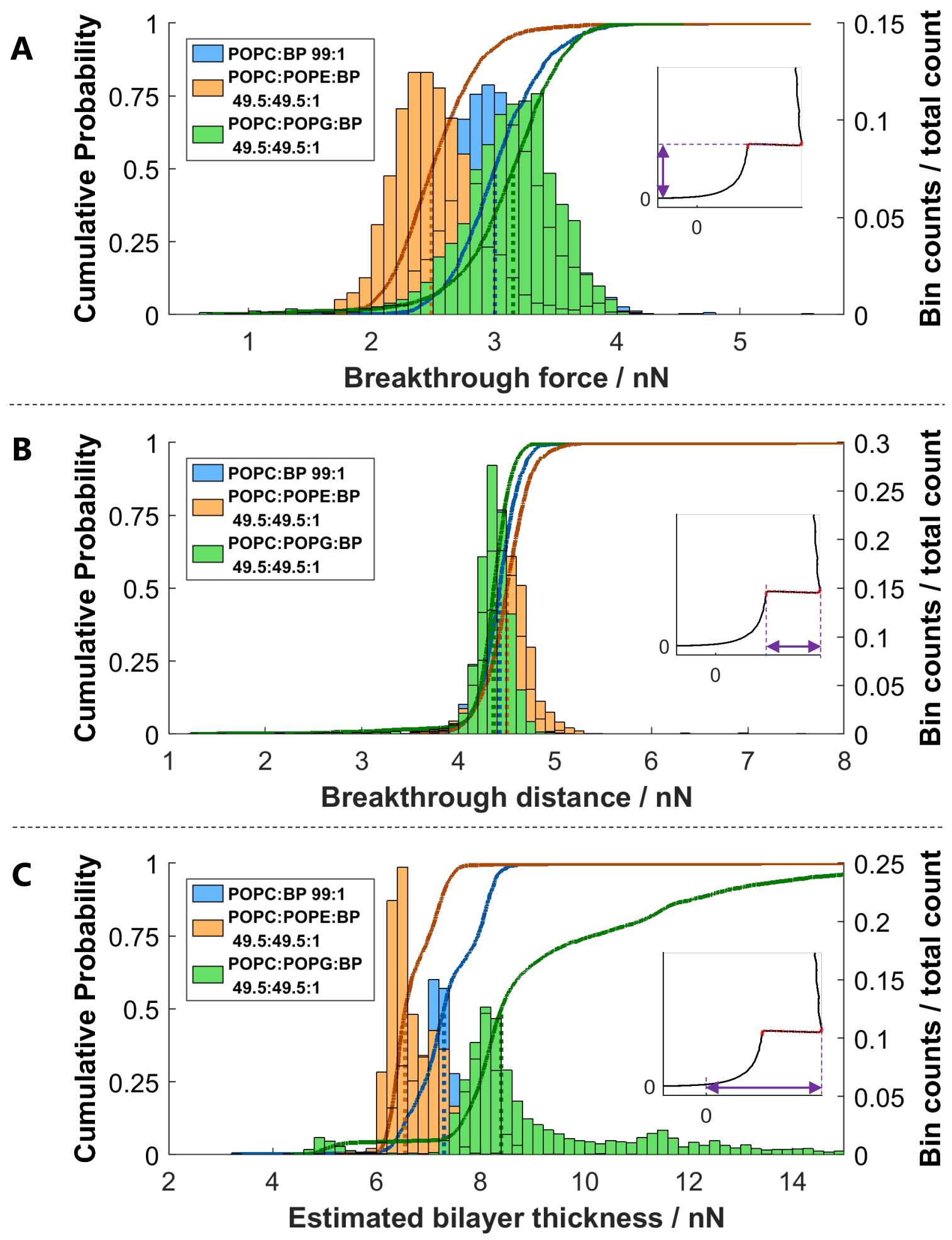

Figure 4.2: Exemplary breakthrough force spectroscopy results for all investigated lipid compositions. Data are presented as both normalised histogram (right $y$-axis) and the corresponding empirical cumulative distribution (left $y$-axis) of the data, with the median of datasets indicated as dotted vertical lines. Note that this median roughly corresponds to the peak in a histogram, and always corresponds to $50 \%$ of the data at or below this value. For clarity, only cumulative plots are shown for individual experiments in Appendix 2-4 p. II-XIX. The inset sample force curve in each figure illustrates the values that were deduced from force curves and plotted in the respective figure. A: Breakthrough forces, defined as the average $y$-coordinate of the points in the detected breakthrough event (red crosses); B: Breakthrough distances, as defined by the range of $x$-coordinates of the points in the breakthrough event; $\mathbf{C}$ : An estimate of the bilayer thickness, defined as the $x$-coordinate of the last point detected in the breakthrough event, relative to the contact point at $\mathrm{x}=0$. See $p .53$ for details on how these values were obtained. 
Breakthrough distance datasets often had large standard deviations (see Appendix 5 p. XXVII. This was mainly attributed to processing artefacts for breakthrough events at low force, which led to some very large values ( $\mu \mathrm{m}$ instead of $\mathrm{nm}$ range). This was partially mitigated by removing values greater than 5 times the median of the normalised and combined datasets, with the reasoning that these were most likely due to either aforementioned processing artefacts, or to vesicles sitting on the membrane (expected diameter $>20 \mathrm{~nm}$ ).

In Figure 4.2 C, exemplary estimated membrane thickness value distributions (breakthrough distance + indentation) for all investigated lipid mixtures are presented. Values were obtained by taking the $x$-coordinate of the last point in the breakthrough event, relative to the pre-set contact point, which was defined as 0. The membrane thickness obtained in this way was very sensitive to measurement artefacts ('skew' of the force curve contact range), as well as the arbitrary contact point determination. Therefore, these values were not analysed further. Figure 4.2 C serves as an illustration of what membrane thickness can be expected or estimated from force curves: 6-10 nm. Literature values from ellipsometry on similar membranes typically show much lower values: ca. 3-4 nm[155-157]. Atomic force microscopy imaging rather shows membrane thickness of $4 \mathrm{~nm}$ and up[105, 156, 157].

\subsubsection{Breakthrough force results with $\mathrm{R}$ - derivatives}

It was expected that the increased likelihood of pore formation as demonstrated in simulations would translate into a lower applied force needed to break through membranes. Breakthrough distance was evaluated, since membrane thinning was observed in simulations.

In each experiment, breakthrough force curves were first collected without the $\mathrm{R}$ derivative. Then, the R-derivative was added and force curves were collected again. Before combination of three repeats of the same experiment or comparison to other experiments, each experimental dataset was normalised for the median of the values collected without the R-derivative.

It was noted that a small rise in salt concentration led to a $10 \%$ decrease in breakthrough force (data in Appendix 1 p. I), while actually an opposite trend 
should be expected based on literature data [107, 158]. Effects of R-derivatives were therefore only assumed when the medians of datasets differed by at least a standard error of the compared datasets, even if differences were statistically significant as per T-test (data not shown).

\section{POPC:BP 99:1}

In Figure 4.3, boxplots of combined and normalised breakthrough force data for POPC membranes are presented, along with the most important statistical parameters for each dataset. A table with more elaborate statistical dataset information can be consulted in Appendix 5 . XXVII. Individual experiments can be found in Appendix 2 p. II.

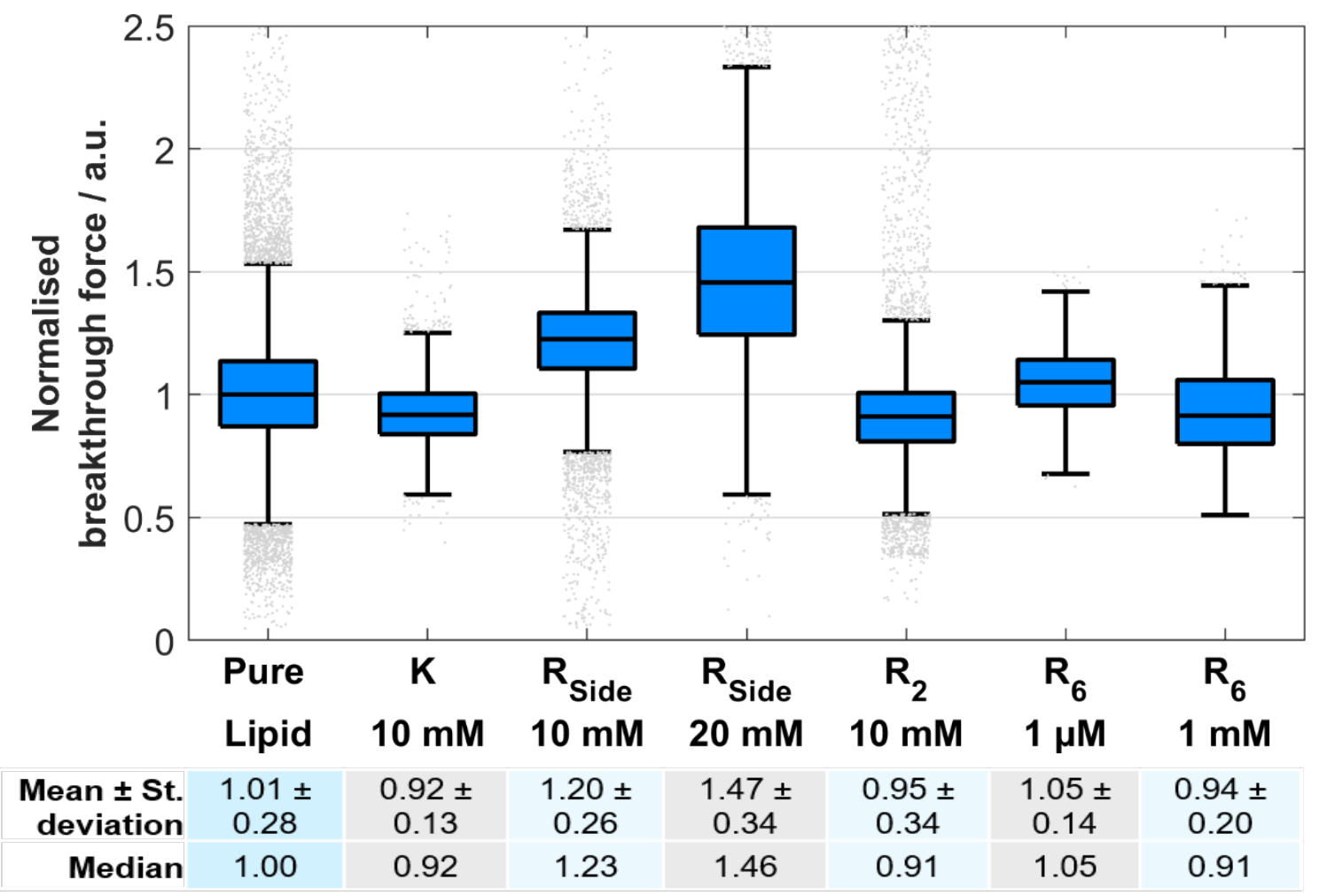

Figure 4.3: Boxplots of normalised breakthrough force results for POPC:BP 99:1. The bottom and top edge of the boxplots indicate the $25^{\text {th }}$ and $75^{\text {th }}$ percentile of each data set, and the line dividing the box indicates the median (also indicated below). The upper and lower whisker represent approximately 2.7 standard deviations higher or lower than the mean, respectively. Outliers (points beyond 2.7 standard deviations from the mean) are shown as grey points. Median, mean and standard deviation for each dataset are given below each boxplot. Significance is assumed if the difference between medians of two datasets is greater than the standard deviations of the compared datasets, which is only the case for $R_{\text {side }}$ $20 \mathrm{mM}$.

Rside shows the clearest influence on breakthrough force in POPC membranes: the data distributions suggest an increase with respect to the pure lipid. This effect seems to be cumulative, as the median for $20 \mathrm{mM} \mathrm{R}_{\text {side }}$ has shifted by about twice 
the amount as that for $10 \mathrm{mM} \mathrm{R}_{\text {side. }}$. The trend was reproducible between experimental repeats (see Appendix 2 p. II Figure A 2.2 and Figure A 2.3).

Therefore, attempts were made to find an explanation for these trends. Literature has shown before that increased ionic strength or total solute concentrations increase breakthrough force[107], increase bilayer packing and can even slow diffusion of headgroup fluorophores[158, 159]. During present experiments, a significant amount of a charged compound was added to the sample, hence there must be an increase of ionic strength. Indeed, osmolarity of a sample used for these experiments typically increased by a factor 1.4 when compared to an unused sample (as probed by freezing point osmometry, data not shown). However, it was reasoned that this could not account for the observed effects on its own, since the other lipid mixtures did not show the same trend for Rside (see $p .71-72$, data for POPC:POPE:BP 49.5:49.5:1 and POPC:POPG:BP 49.5:49.5:1).
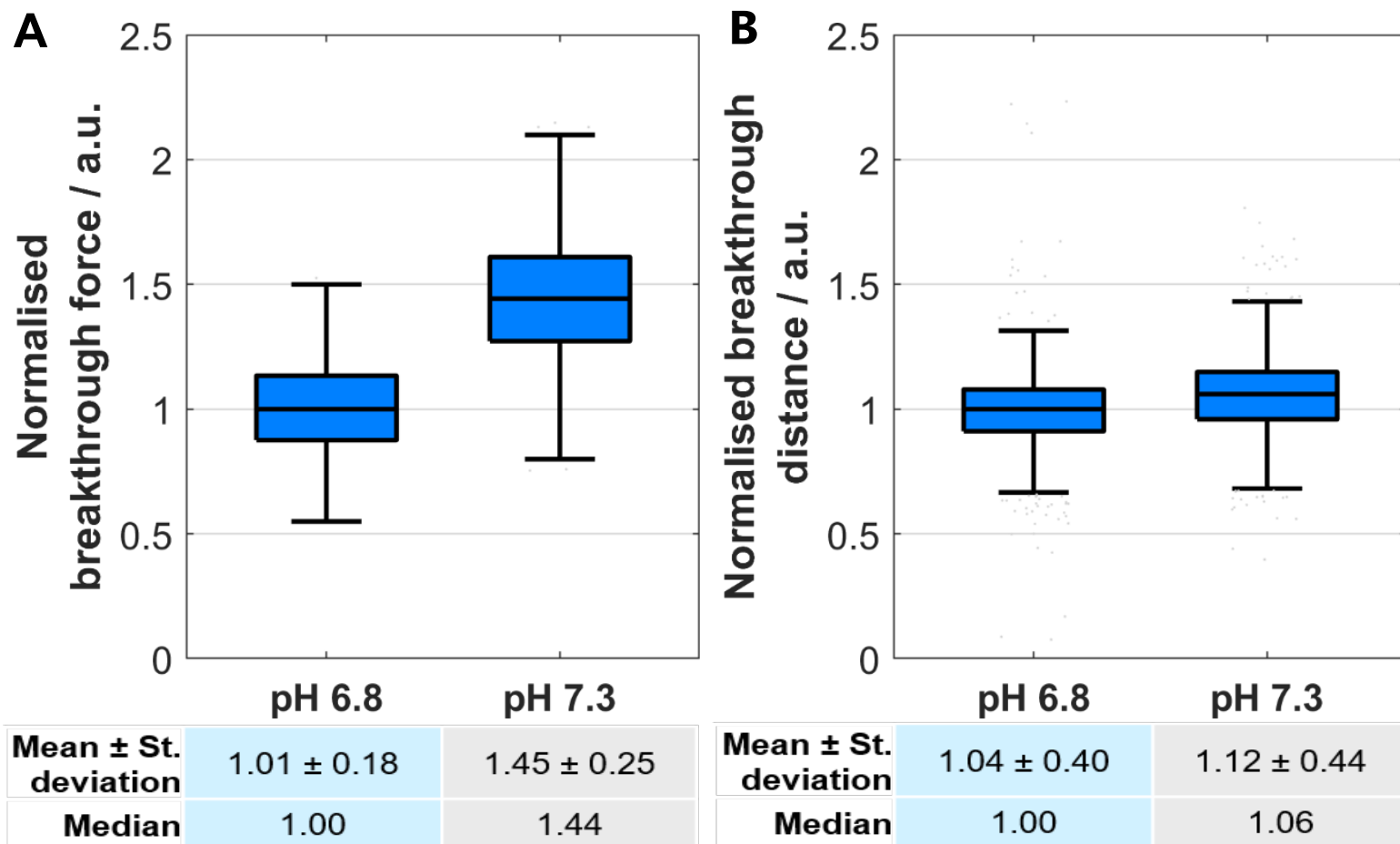

Figure 4.4: Normalised boxplot results of a breakthrough force experiment on POPC:BP 99:1 membranes during which the sample buffer was exchanged for one with a higher $\mathrm{pH}$ (6.8 to 7.3). The bottom and top edge of the boxplots indicate the 25th and 75th percentile of each data set, and the line dividing the box indicates the median. The upper and lower whisker represent approximately 2.7 standard deviations higher or lower than the mean, respectively. Outliers are shown as grey points. Median, mean and standard deviation for each dataset are given below each boxplot. Effects of R-derivatives were assumed if the difference between medians of two datasets was greater than a typical standard deviation. A: breakthrough force results; B: breakthrough distance results. 
Next, the effect of $\mathrm{pH}$ was investigated. Qualitative analysis of the $\mathrm{pH}$ (data not shown) of rest solutions from closed-system RIfS experiments on POPG-containing membranes (see Sections 3.6 p. $55 \mathrm{ff}$. and 4.5 .1 p. $81 \mathrm{ff}$.) suggested that $\mathrm{R}_{\text {Side }}$ addition could increase the $\mathrm{pH}$ in the sample from ca. 6.8 up to ca. 7.4 at higher concentrations.

Therefore, a breakthrough force experiment was performed with a buffer exchange from $\mathrm{pH} 6.8$ to $\mathrm{pH}$ 7.3. Normalised results are shown in Figure 4.4, for easier comparison to Figure 4.3. The same trend seems to be visible as for $20 \mathrm{mM} \mathrm{R}_{\text {side }}$ addition to this lipid mixture: a significant upwards shift of the breakthrough force median, simultaneous with a broadening of the data distribution, but not as pronounced a breakthrough distance change (see Section 4.2.3 p. 74 ff.). 


\section{POPC:POPE:BP 49.5:49.5:1}

Breakthrough force results of experiments on a POPE-containing lipid mixture are shown in Figure 4.5, again in normalised fashion and as boxplots. For none of the tested R-derivatives and concentrations a clear permanent change could be observed. In case of Rside, a temporary change (scale of an hour) could be observed in some individual experiments, where the last force map data showed a (near) return to initial pure lipid values (data not shown). However, this occurred both in downward and in upward direction, and was therefore considered a temporary artefact due to cantilever tip changes or particle adhesion.

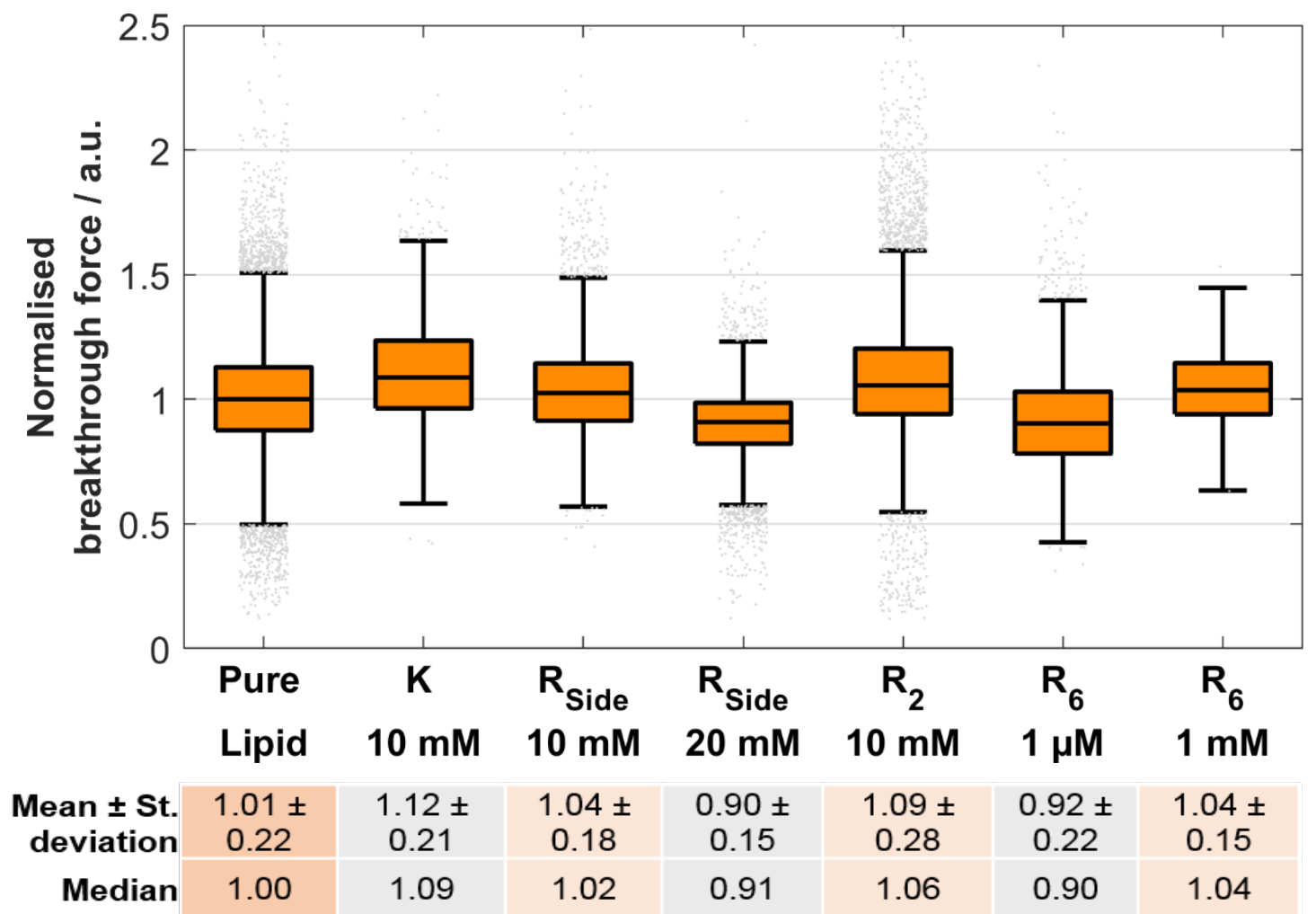

Figure 4.5: Boxplots of normalised breakthrough force results for POPC:POPE:BP 49.5:49.5:1. The bottom and top edge of the boxplots indicate the $25^{\text {th }}$ and $75^{\text {th }}$ percentile of each data set, and the line dividing the box indicates the median (also indicated below). The upper and lower whisker represent approximately 2.7 standard deviations higher or lower than the mean, respectively. Outliers are shown as grey points. Median, mean and standard deviation for each dataset are given below each boxplot. Significance is assumed if the difference between medians of two datasets is greater than a typical standard deviation, which is not the case for any of the datasets shown here. 


\section{POPC:POPG:BP 49.5:49.5:1}

Breakthrough force results for POPG-containing bilayers are shown in Figure 4.6. In this case, significant reduction of breakthrough force was observed for $\mathrm{R}_{\text {side }}$ in both concentrations, and to a lesser extent for $R_{2}$. Both concentrations of $R_{\text {side }}$ led to the same $\sim 40 \%$ decline in breakthrough force. The susceptibility of POPG to influence by $\mathrm{R}_{\text {Side }}$ is shared by the simulation results. However, there was no clear difference observed for the two different Rside concentrations. This was unlike the simulations, in which doubling the number of $\mathrm{R}_{\text {side }}$ units led to stronger changes to measured parameters such as area per lipid and pore nucleation energy (see Section 4.1 p. 62 ff.). For $R_{6}$, much weaker effects were observed, not even for the highest feasible concentration of $1 \mathrm{mM}$, despite significant breakthrough force reduction by $R_{2}$.

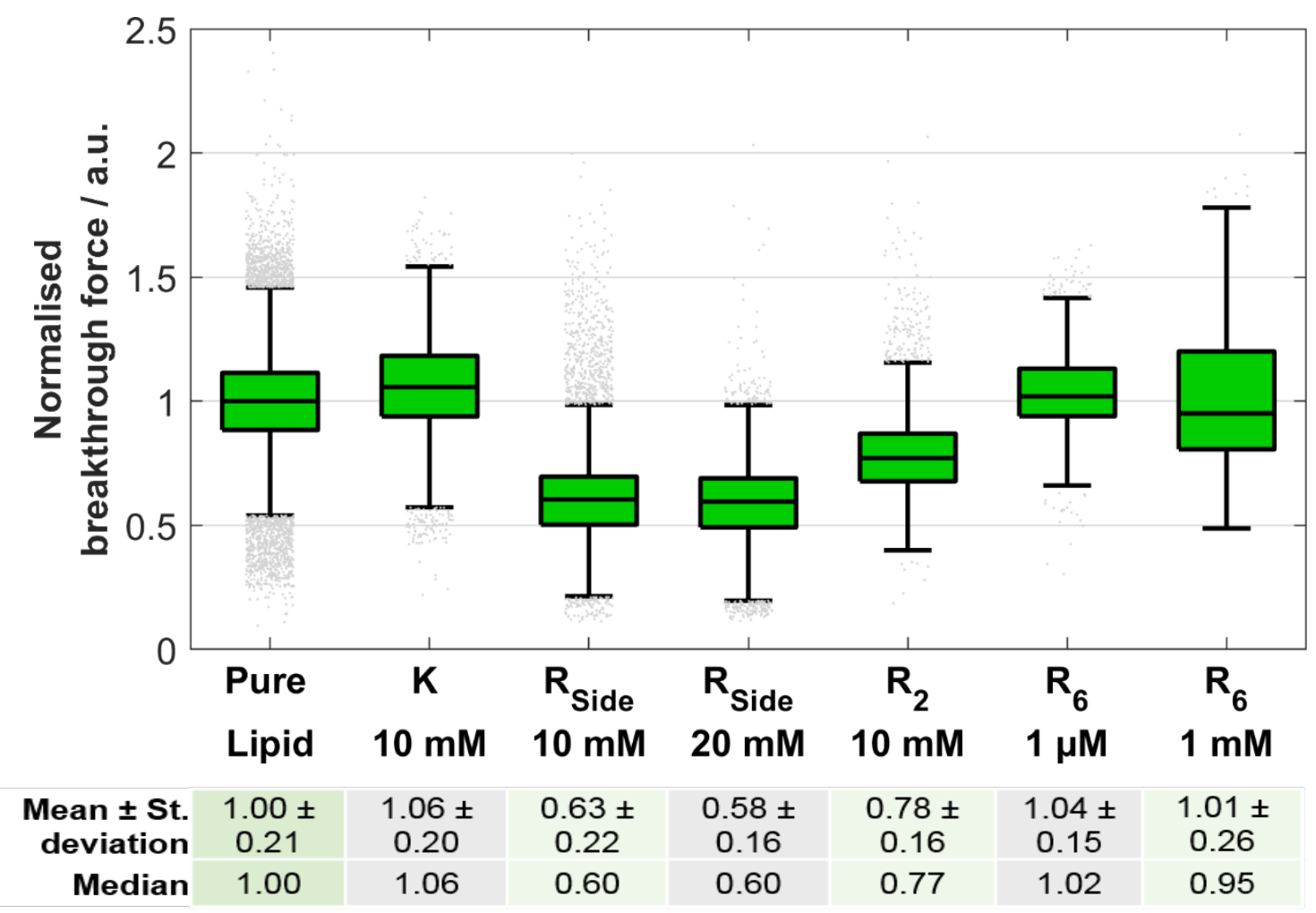

Figure 4.6: Breakthrough force results for POPC:POPG:BP 49.5:49.5:1 lipid mixtures combined with Rderivatives. The bottom and top edge of the boxplots indicate the $25^{\text {th }}$ and $75^{\text {th }}$ percentile of each data set, and the line dividing the box indicates the median (also indicated below). The upper and lower whisker represent approximately 2.7 standard deviations higher or lower than the mean, respectively. Outliers are shown as grey points. Median, mean and standard deviation for each dataset are given below each boxplot. Effects of R-derivatives were assumed if the difference between medians of two datasets was greater than a typical standard error. This is the case for both $R_{\text {side }}$ concentrations and $R_{2}$.

However, on the force curve level, an observation could be made for higher concentrations of $R_{6}$, that was not observed for other R-derivatives: the onset of 
the indentation part of the approach curve showed a brief negative force before the force increased. This so-called jump-to-contact or 'snap-on' is characteristic for short-range attractive forces between cantilever tip and sample [115]. Figure 4.7 shows characteristic onsets of indentation curves at three different concentrations of $R_{6}$ in POPG-containing membranes, as compared to typical curves for the pure lipid (Figure 4.7 A). At $1 \mu \mathrm{M}$ concentration (Figure 4.7 B), indentation looks very similar to force curves for the pure lipid. At a $100 \mu \mathrm{M} \mathrm{R}_{6}$ concentration (Figure 4.7 C), part of the force curves show a jump-to-contact. At a $1 \mathrm{mM} \mathrm{R}_{6}$ concentration, a very reproducible and discrete jump-to-contact occurs (Figure 4.7 D).

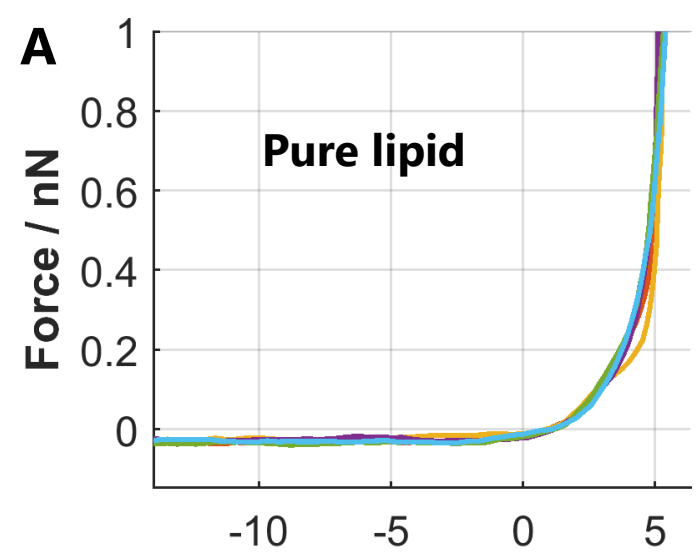

Separation / $\mathrm{nm}$

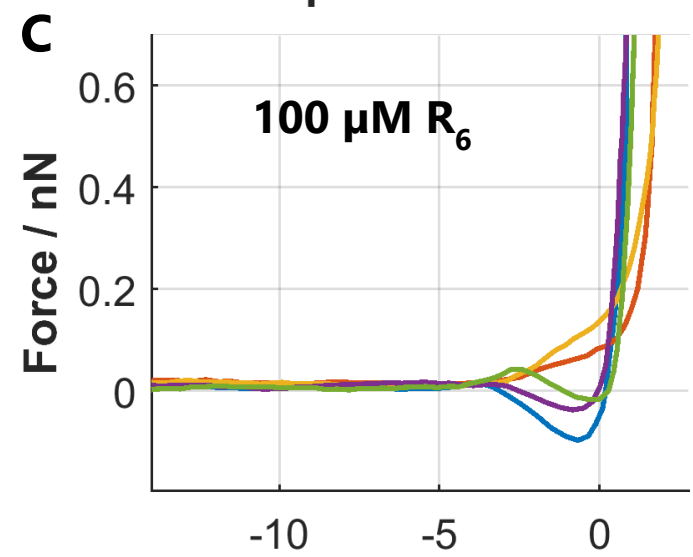

Separation / $\mathrm{nm}$

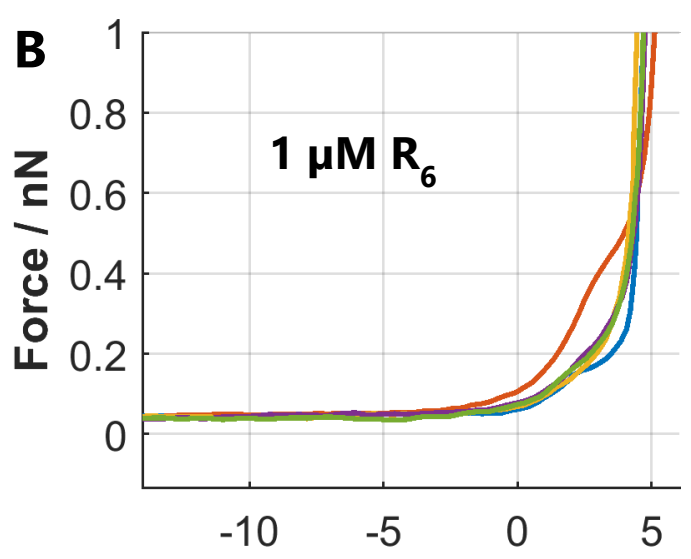

Separation / nm

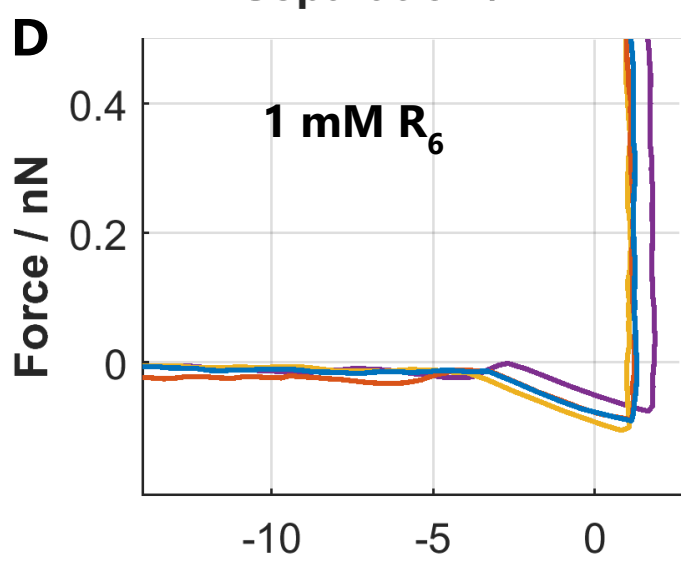

Separation / $\mathrm{nm}$

Figure 4.7: Exemplary initial force-separation curves of POPG-containing lipid bilayers with several concentrations of $R_{6}$, Colours serve to discriminate different force curves. A: pure lipid; $\mathbf{B}: 1 \mu \mathrm{M} \mathrm{R}_{6} ; \mathbf{C}$ : $100 \mu \mathrm{M} \mathrm{R}_{6}$ and $\mathbf{D}: 1 \mathrm{mM} \mathrm{R}_{6}$.

This could be explained by assuming that in these experiments, lipid material is often deposited on the cantilever tip (reasonable since tether pulling was often observed, data not shown). Since the membrane is negatively charged through its hight PG content, this would cause a repulsive effect between lipid-covered 
cantilever tip and the solid-supported membranes. If the planar membrane is covered with positively charged arginine oligopeptide, this would promote attraction rather than repulsion; the positively charged oligopeptide screens the negative charge and also attracts the lipid-covered cantilever tip.

\subsubsection{Breakthrough distance results with $\mathrm{R}$ - derivatives}

Breakthrough distance results were analysed with the purpose of confirming the membrane thinning effect of R-derivatives that was observed in simulations (Section 4.1 p. $62 \mathrm{ff}$.). Such an effect was not expected to be straightforwardly deducible from these data, since breakthrough distance does not encompass the full membrane thickness (see Section 2.2.1 p. $33 \mathrm{ff}$.). However, it is possible that differences in membrane properties as induced by $\mathrm{R}$-derivatives change the ratio between indentation depth and breakthrough event distance. Therefore, these data were analysed.

Figure $4.8(p .74)$ shows boxplots of breakthrough distance results for all lipids and all R-derivatives. No significant changes were observed for any combination. Statistical quantities for these results can be found in Appendix 5 p. XXVII, and absolute data for all experiments are given in Appendix 2-4 p. II-XIX. 

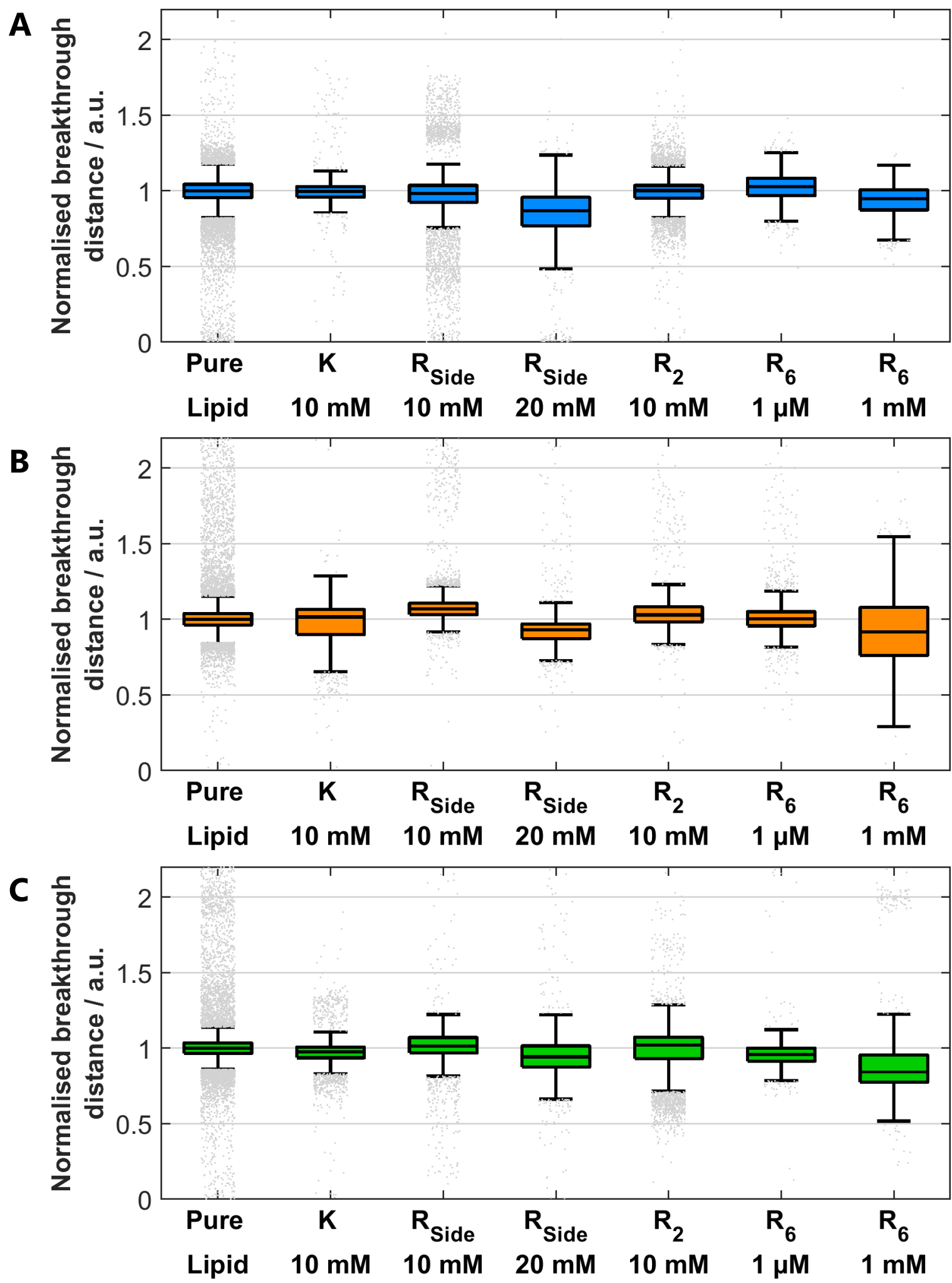

Figure 4.8: Boxplots of breakthrough distance results for all lipids and all R-derivatives. The bottom and top edge of the boxplots indicate the $25^{\text {th }}$ and $75^{\text {th }}$ percentile of each data set, and the line dividing the box indicates the median (also indicated below). The upper and lower whisker represent approximately 2.7 standard deviations higher or lower than the mean, respectively. Outliers are shown as grey points. Median, mean and standard deviation for each dataset are given below each boxplot. Significance is assumed if the difference between medians of two datasets is greater than a typical standard deviation. In none of the compared datasets, this is the case. A: POPC:BP 99:1; B: POPC:POPE:BP 49.5:49.5:1; C: POPC:POPG:BP 49.5:49.5:1. 


\section{$4.3 \mathrm{R}_{6}$-induced liposome leakage}

Vesicle leakage induced by nona-arginine $\left(R_{9}\right)$ was shown by Allolio et al. [11]. It was therefore investigated whether $R_{6}$ could induce lipid vesicle leakage, if not compromise membrane integrity in previously shown breakthrough force experiments. Allolio et al. conducted vesicle studies with a mixture of DOPE and DOPS and concluded PE headgroups were the most important for $R_{9}$ action[11]. The present study's data suggest, however, that PS headgroups are more likely the crucial factor (see Chapter 5 p. 91 ff.). To investigate this, leakage assays were also performed on 1:1 mixtures of either POPS or POPE with POPC.
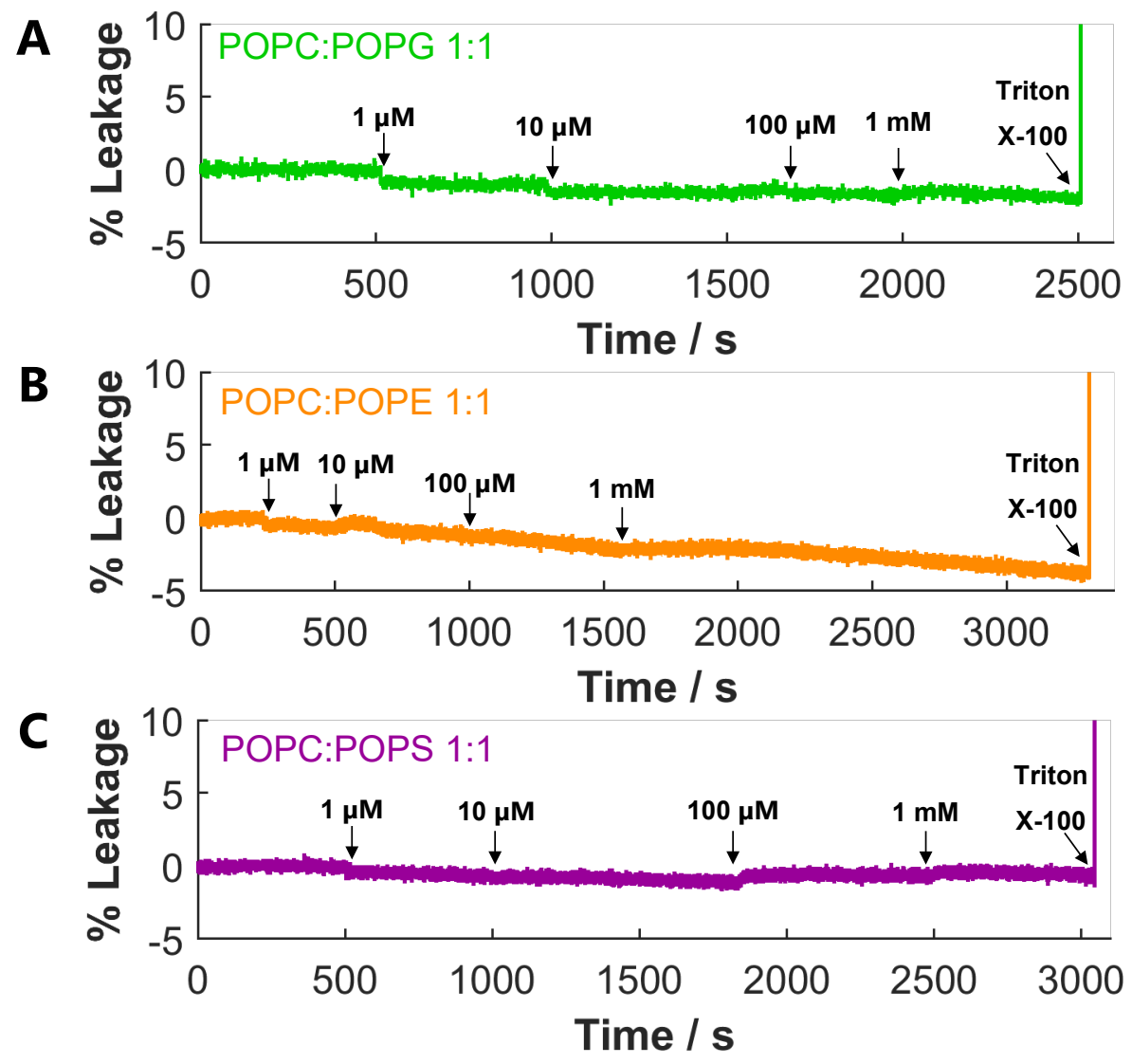

Figure 4.9: Leakage assays of sulforhodamine $B$ in vesicles of three different compositions. Increasing concentrations of $R_{6}$ were added to the solution and fluorescence intensity was left to stabilise. Fluorescence intensity at $585 \mathrm{~nm}\left(\lambda_{\mathrm{ex}}=565 \mathrm{~nm}\right)$ was monitored, and normalised to $0 \%$ for vesicles before $\mathrm{R}_{6}$ addition, and to $100 \%$ for the fluorescence value detected after Triton X-100 addition (plateau out of range of plots). Data were corrected for linear increase or decay by subtracting a line fitted through the data before $\mathrm{R}_{6}$ addition. Additions are indicated with arrows and the corresponding concentration. This assay was conducted under the same conditions as the solid-supported bilayer experiments (phosphate buffer). Hence it was not possible to determine exact peptide-to-lipid ratios by means of a phosphate test. However, peptide-to-lipid ratios were estimated to be around 1:19-57:1. A: POPC:POPG 1:1; B: POPC:POPE 1:1; C: POPC:POPS 1:1. 
Leakage assays were performed by loading vesicles with buffer containing the selfquenching dye sulforhodamine $B$, and monitoring its fluorescence by adding increasing amounts of $R_{6}$ peptide to the mixture. If vesicle integrity is compromised, sulforhodamine B is diluted into the bulk of the solution, and hence it is freed of its self-quenching state; its fluorescence intensity increases. Data are normalised to $100 \%$ leakage, for the fluorescence intensity value after addition of Triton X 100, which ensues complete destruction of vesicles in solution. It was chosen to conduct this assay under the same conditions as the solid-supported bilayer experiments (phosphate buffer). Hence it was not possible to determine exact peptide-to-lipid ratios. However, peptide-to-lipid ratios were estimated to be around $1: 19$ for $1 \mu \mathrm{M}$ peptide up to $57: 1$ for $1 \mathrm{mM}$ peptide concentrations.

First, leakage of vesicles containing POPG was investigated (Figure 4.9 A), since PG-containing membranes showed the highest susceptibility to change in previously shown breakthrough force experiments. No significant increase of fluorescence was detected even for higher $\mathrm{R}_{6}$ concentrations, and hence no leakage caused by $R_{6}$. A small upwards step occurred when concentration was increased to $1 \mathrm{mM}$, while at lower concentrations a downwards step occurred, presumably due to dilution.

A leakage assay in Hepes buffer with carboxyfluorescein and determined peptideto-lipid ratio of $1: 1.38$ (data in Appendix $6 p$. XXIX) yielded a leakage of about $5.4 \%$. At least $10 \%$ leakage is usually expected for a compound to be classified as leakage-inducing in literature describing bulk leakage assays in liposomes [160164]. Furthermore, Allolio et al. measured $82 \%$ leakage for $\mathrm{R}_{9}$ at a much lower peptide-to-lipid ratio of 1:66 (see supporting information to [11]).

Figure 4.9 B and $\mathbf{C}$ show the leakage assays for POPE- and POPS- containing vesicles, respectively. Neither for these lipid mixtures, any significant leakage of sulforhodamine B was detected. For POPS, a small upwards step was detected for both $100 \mu \mathrm{M}$ and $1 \mathrm{mM}$, while for POPE no such discrete upwards steps were observed. 


\subsection{Membrane fluidity}

Intuitively, it can be expected that reduction in breakthrough force, as observed for POPG-containing membranes with $\mathrm{R}_{\text {side, }}$ could correlate with an increase in membrane fluidity. Therefore, fluorescence recovery after photobleaching (FRAP) experiments were conducted, as a measure of membrane fluidity. Diffusion

A

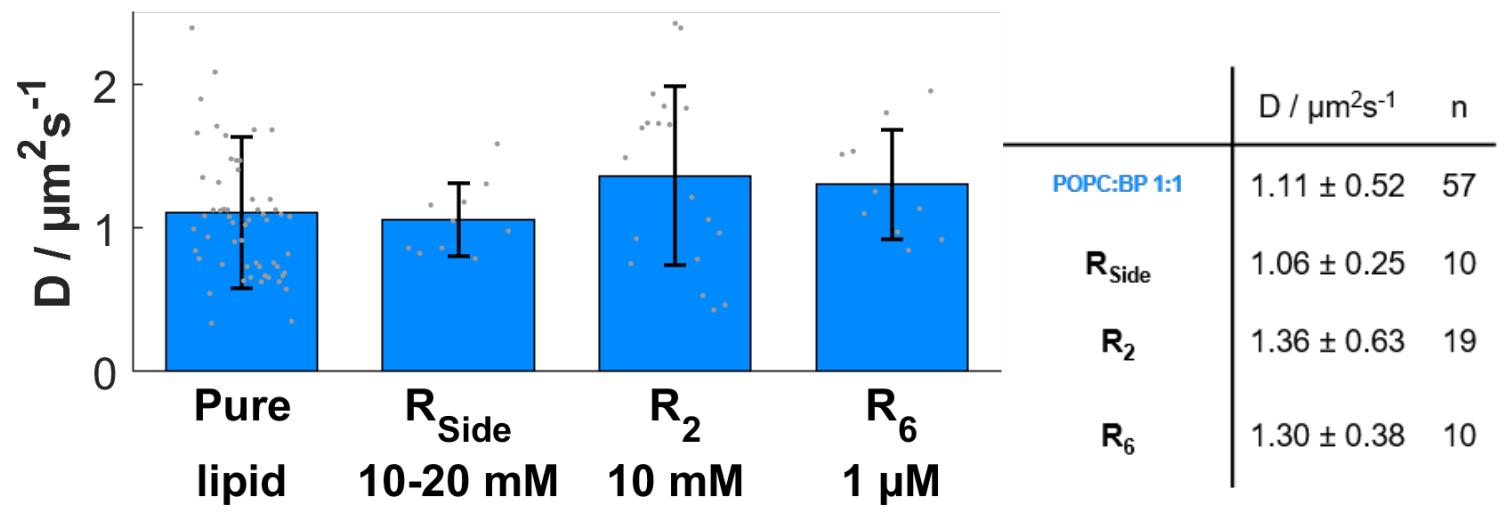

B

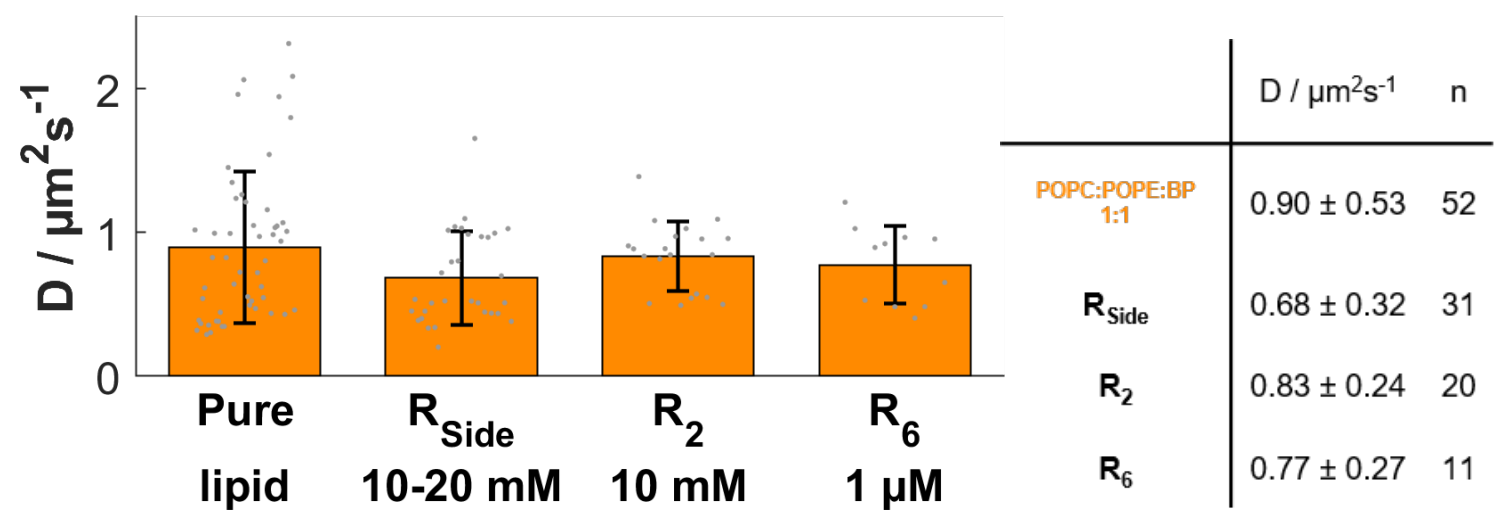

C

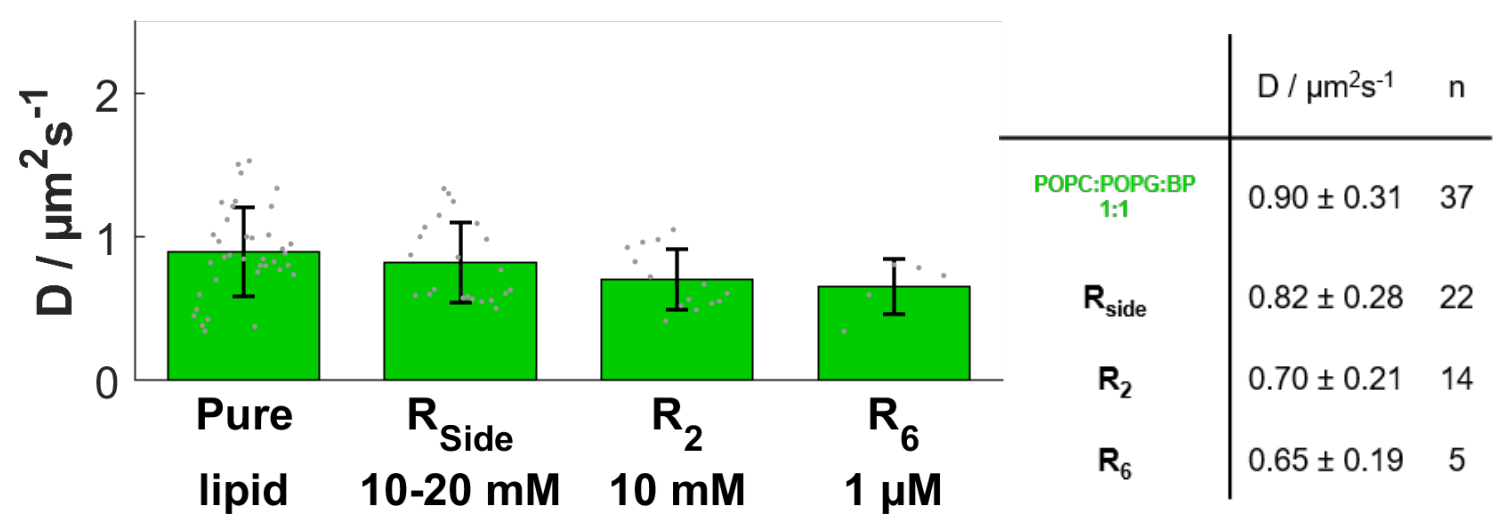

Figure 4.10: Diffusion constants of BP for all lipids, as well as each lipid combined with all investigated R-derivatives. Bars and errorbars represent mean diffusion constants and their standard deviations, respectively. Individual values are shown as grey scatter points. Mean and standard deviation, as well as number of measurements in each plot, is shown to the right. Values for both Rside concentrations were combined, since they did not show significant difference. A: POPC:BP 99:1; B: POPC:POPE:BP 49.5:49.5:1; C: POPC:POPG:BP 49.5:49.5:1. 
coefficients of BP with and without each R-derivative were determined from FRAP experiments, and are shown for each lipid composition in Figure $\mathbf{4 . 1 0}$ (data combined from at least two experiments). For each lipid composition, the diffusion

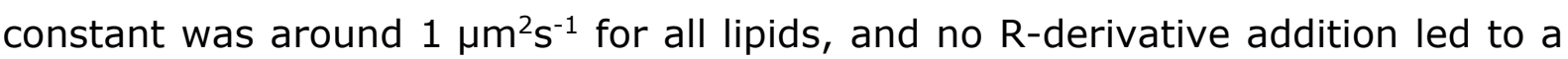
significant change of the diffusion coefficient.

\subsection{Visual effects of R- derivatives}

Besides determination of diffusion coefficients, confocal laser scanning microscopy as well epi-fluorescence microscopy was employed to observe visual changes to the solid-supported lipid bilayers upon addition of R-derivatives.

Figure 4.11 shows exemplary fluorescence micrographs for all investigated lipid compositions without R-derivatives. In case of POPG-containing membranes, a homogeneous bilayer could be reproducibly obtained (Figure 4.11 A, left image). Tube formation upon longer microscopic observation was however common in these membranes, along with larger round structures (Figure 4.11 A, right image). For both POPC-only membranes and the 1:1 mixture with POPE, small dots on or close to the surface were reproducibly observed (Figure 4.11 B \& C). Since many of these dots were very mobile (data not shown), it was assumed that these dots were vesicles, either adhered to the membrane surface (immobile) or in solution but retained closely to the surface. These could not be removed with extensive rinsing, neither could they be avoided with changes to the preparation procedure (extrusion of vesicles, vesicle and membrane preparation at elevated temperatures).

For $\mathrm{K}$ and $\mathrm{R}_{2}$, no changes to the membrane were observed for any of the lipid compositions at the used concentrations (10 mM, data not shown). Therefore, only fluorescence results under influence of $R_{\text {side }}$ and $R_{6}$ will be shown here. 

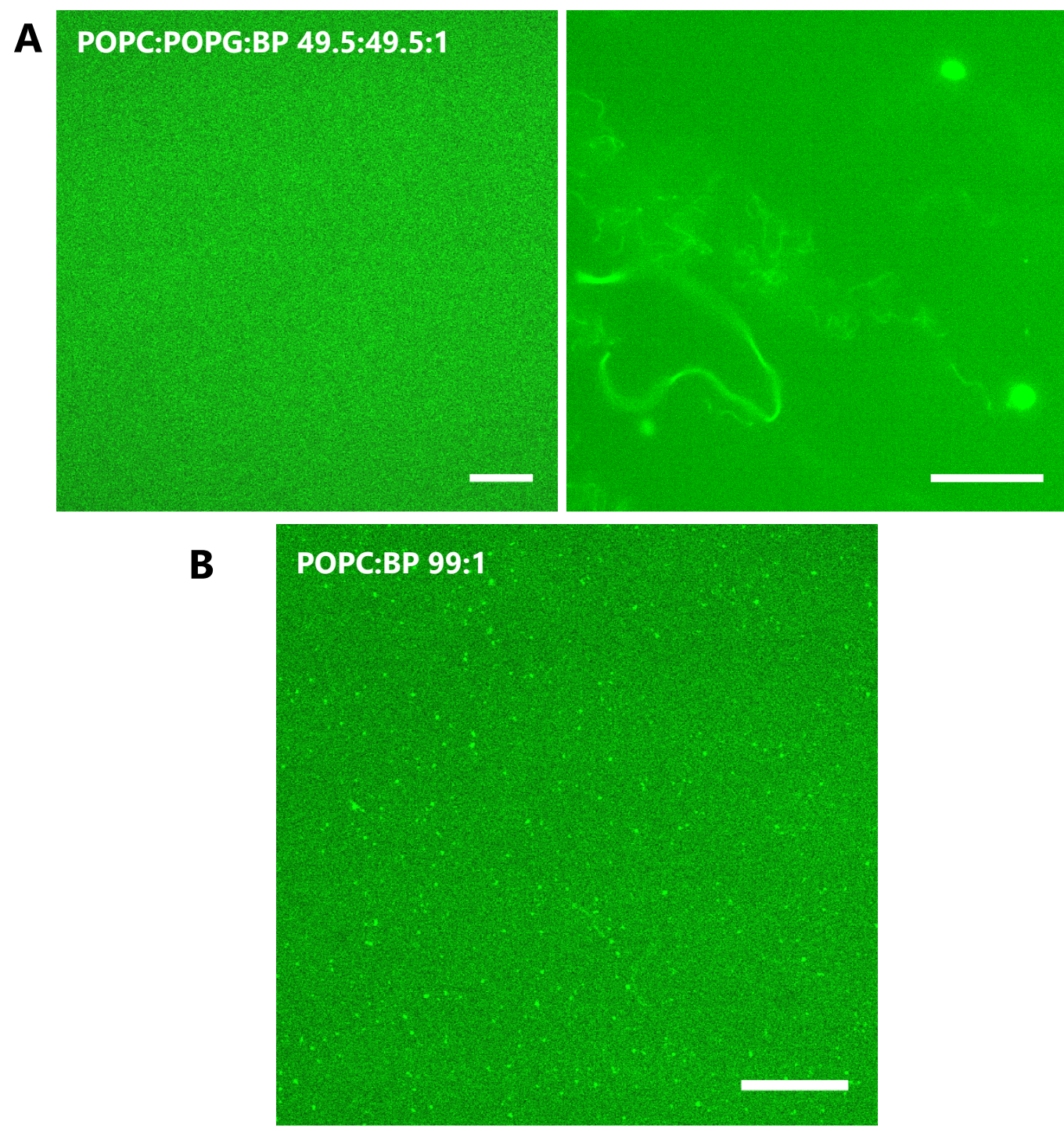

C POPC:POPE:BP 49.5:49.5:1.

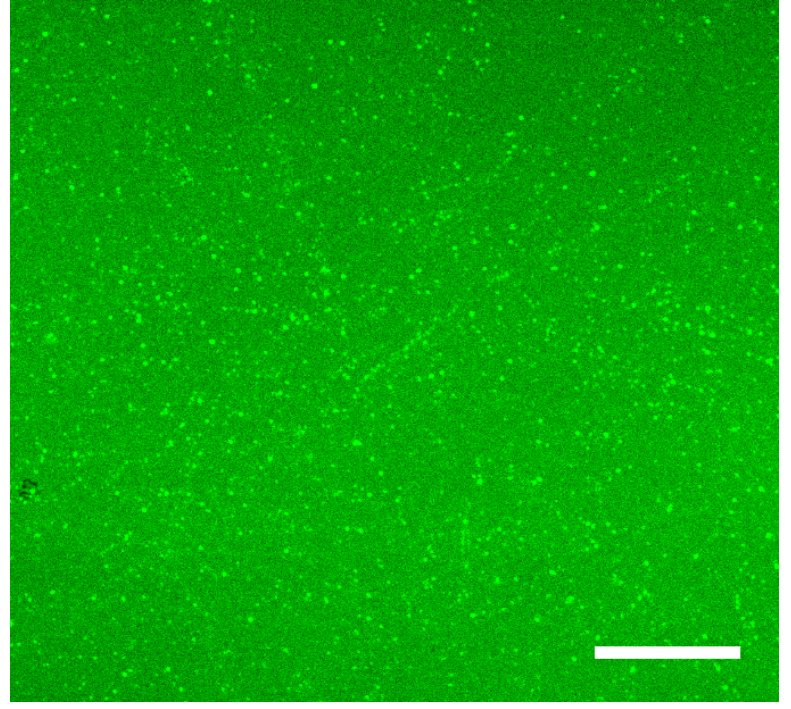

Figure 4.11: Exemplary fluorescence micrographs of all lipid compositions. A: POPC:POPG:BP 49.5:49.5:1, initially (left) and after heating and cooling from microscopy examination (right); B: POPC: BP 99:1; C: POPC:POPE:BP 49.5:49.5:1. Scale bars $20 \mu \mathrm{m}$. 


\subsubsection{Effects of Rside}

Figure 4.12 depicts exemplary series of fluorescence micrographs recorded briefly

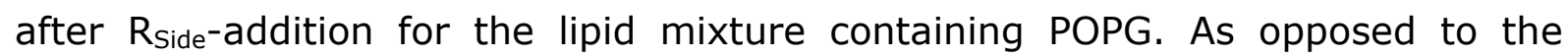
homogeneous membrane shown in Figure 4.11 A, darker 'domains' appeared, as well as apparent vesicles (partially mobile dots and circles). Diffusion in the darker domains was not markedly slower than that in the rest of the membrane (data in Appendix 7 p. XXIX). Within the darker domains, areas that are free of dye seem to expand over time. Larger vesicles (too large to be SUVs) seem to detach from the membrane and drift away, as illustrated for two vesicles in Figure 4.12 B. This could indicate that lipid material is leaving the membrane.

For the 1:1 mixture with POPE, areas free of dye seem to appear but they resolve again. This was also observed once for POPC-membranes but not reproducibly. Beside larger 'holes', also small round holes appear in POPE-containing membranes, but disappear again (Figure 4.13 A, last two images show partial disappearance). POPC-membranes anecdotally showed temporary dark domain formation with $\mathrm{R}_{\text {side, }}$ without the smaller round holes (data in Appendix 11 Figure A 11.2 p. XXXIV). After more time had elapsed (30 min, data not shown), neither of these membranes had a different appearance from their typical appearance shown in Figure 4.11 B and C. 

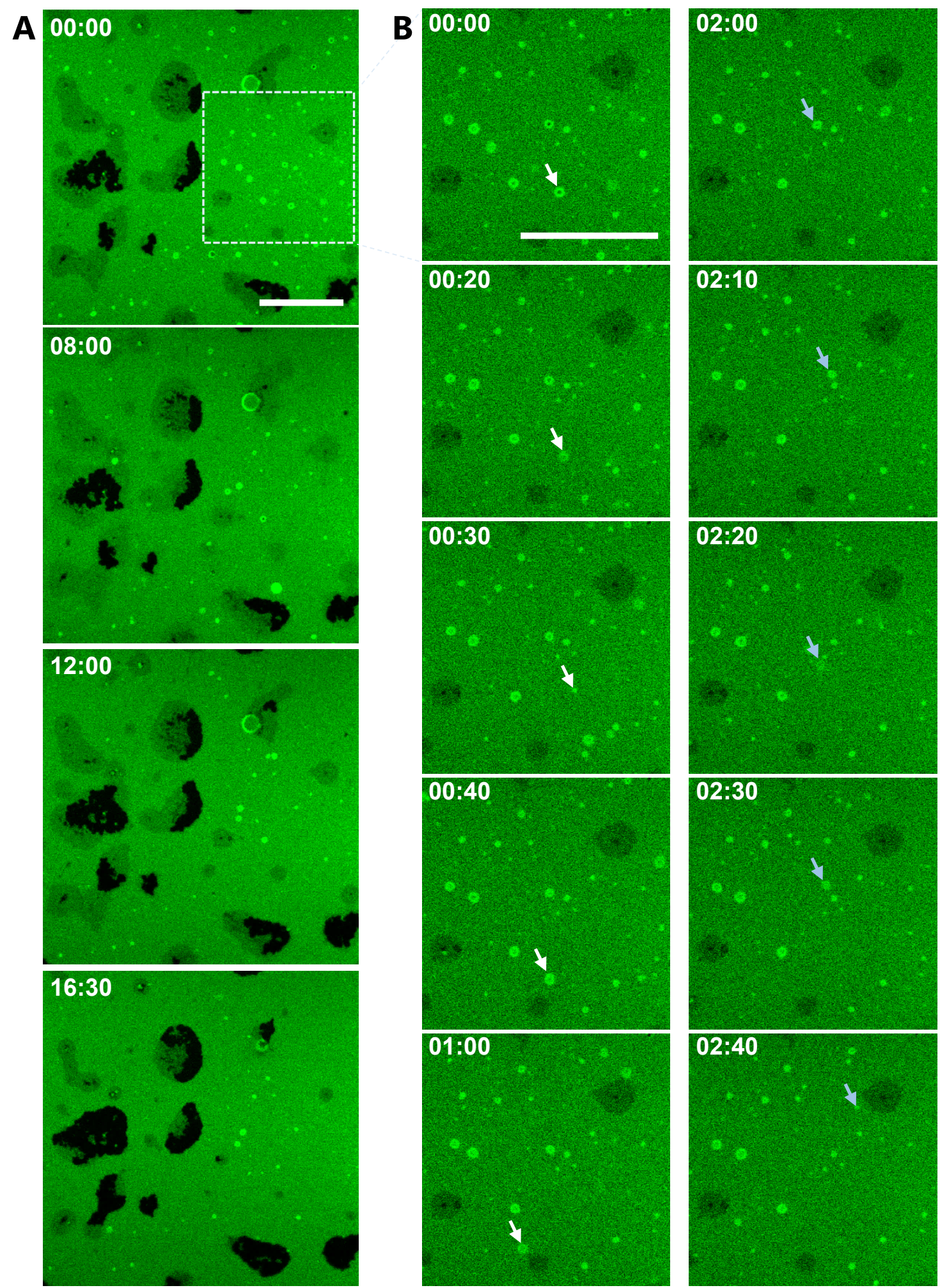

Figure 4.12 A: Series of fluorescence micrographs of a POPC:POPG:BP 49.5:49.5:1 bilayer recorded

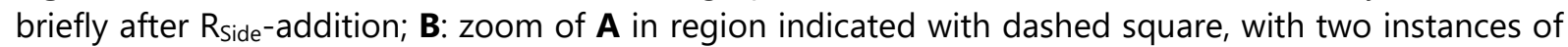
suspected vesicle budding indicated with arrows (white in left column \& light blue in right column, respectively). Time format minutes:seconds, scale bars $20 \mu \mathrm{m}$. 
POPC:POPE:BP 49.5:49.5:1
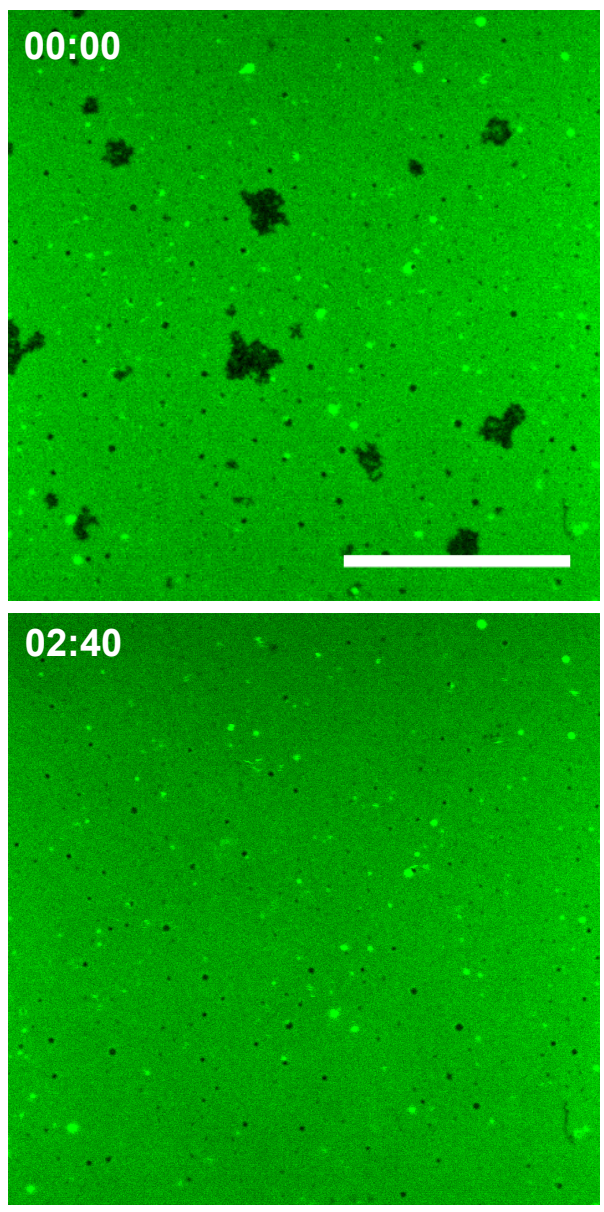

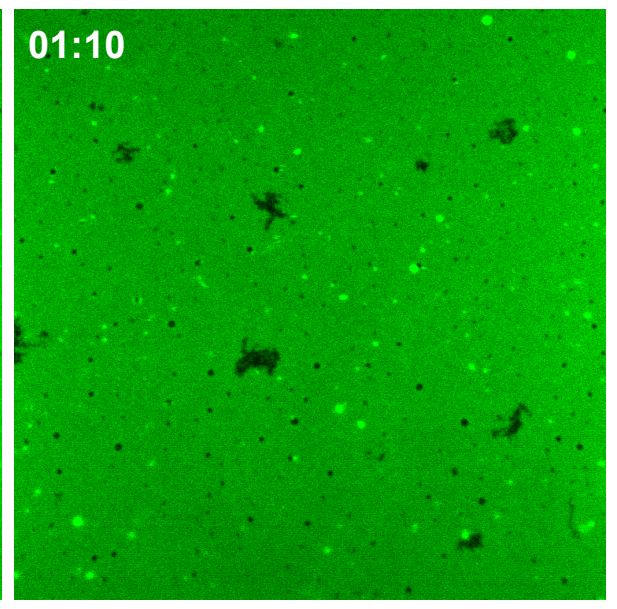

06:20

Figure 4.13: Exemplary fluorescence micrograph series of a POPC:POPE:BP 49.5:49.5:1 membrane recorded after $\mathrm{R}_{\text {side }}$ addition (10 mM). Time in minutes:seconds, scale bar $20 \mu \mathrm{m}$.

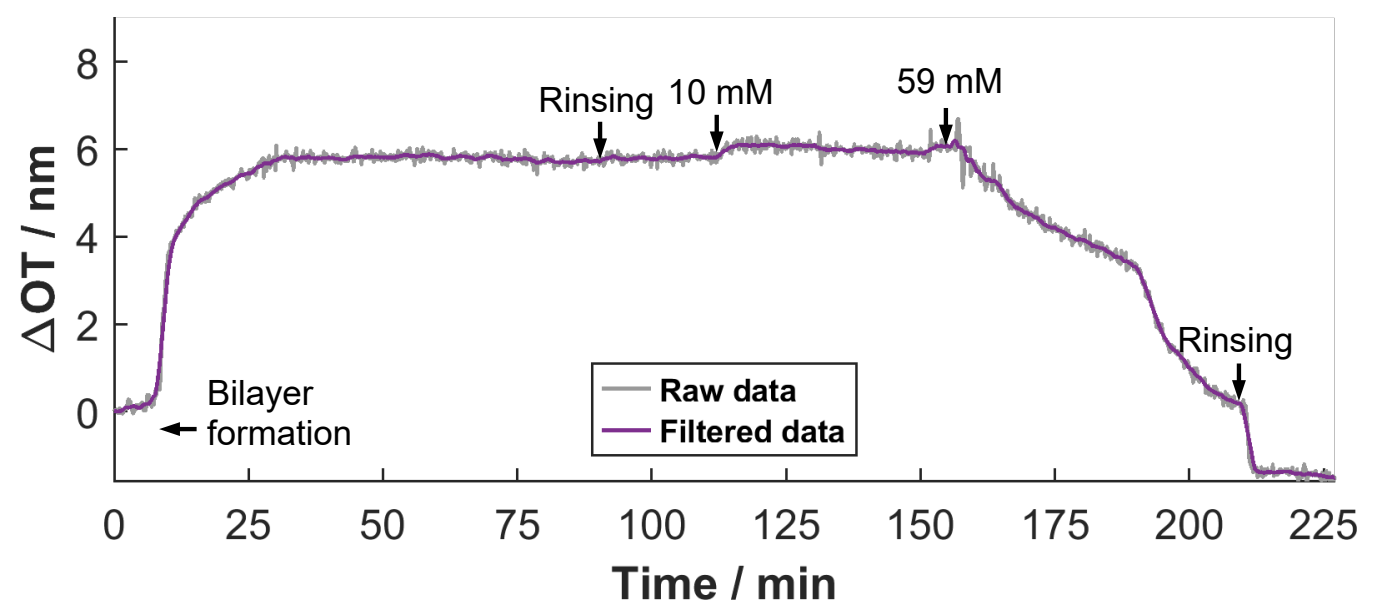

Figure 4.14: Plot of optical thickness against time, for a RIfS experiment on a POPC:POPG:BP 49.5:49.5:1 membrane with increasing concentrations of $R_{\text {side. }}$. The purpose was determining a dissociation constant for $R_{\text {side, }}$ but the bilayer was already destroyed by the second $R_{\text {side }}$ concentration. Data were filtered by a moving mean window (purple curve). 
The nature of black domains and smaller round domains was not further investigated. However, an attempt to determine the dissociation constant of Rside on a POPC:POPG:BP 49.5:49.5:1 membrane by reflectometric interference spectroscopy shed more light on this process (Figure 4.14). First, the membrane was formed on the substrate by adding POPG-containing vesicles, leading to an optical thickness increase of $6 \mathrm{~nm}$. This was typically observed for this membrane (see Chapter 5.1 p. 91). When the Rside-concentration was increased to $59 \mathrm{mM}$, the optical thickness decreased again, a clear indication that the membrane disintegrated. This observation makes it plausible that the dark 'holes' observed in fluorescence are indeed defects caused by lipid material being withdrawn from the membrane. This would mean that the small round holes and the larger vesicle-like structures are most likely the results of vesicle budding.

\subsubsection{Effects of $R_{6}$}

No changes to fluorescence images were observed for POPC and for POPEcontaining membranes, at any concentration of $\mathrm{R}_{6}$ (up to $1 \mathrm{mM}$, data not shown). For POPC:POPG:BP 49.5:49.5:1 membranes, however, patches with higher fluorescence intensity appeared on the membrane. This already occurred at $R_{6}$ concentrations as low as $1 \mu \mathrm{M}$. Most observed patches were approximately $10 \mu \mathrm{m}$ to a few tens of micrometres in size (Figure 4.15 A \& D), but could occasionally span $100 \mu \mathrm{m}$ to several hundreds of micrometres (example in Figure 4.15 C).

Furthermore, higher-intensity-patches appeared to occur at multiple intensity levels. Up to three levels of intensity were observed (Figure 4.15 B). Intensity levels of patches (henceforth referred to as 'levels' only) relative to global membrane fluorescence intensity were indicated in the histograms below the micrographs in Figure 4.15. The relative difference between intensity levels seemed fairly constant within one image (0.5-0.7 relative units).

While patches of different intensity levels often overlapped (Figure 4.15 B-C), homogeneous patches of the second intensity level were often observed was well (Figure 4.15 D). The overlapping and multiple levels of patches seemingly 'growing' on each other, suggested that the observations presented the formation of membrane stacks. Further results concerning the characterisation of patches and their formation process will be presented in the next chapter. 

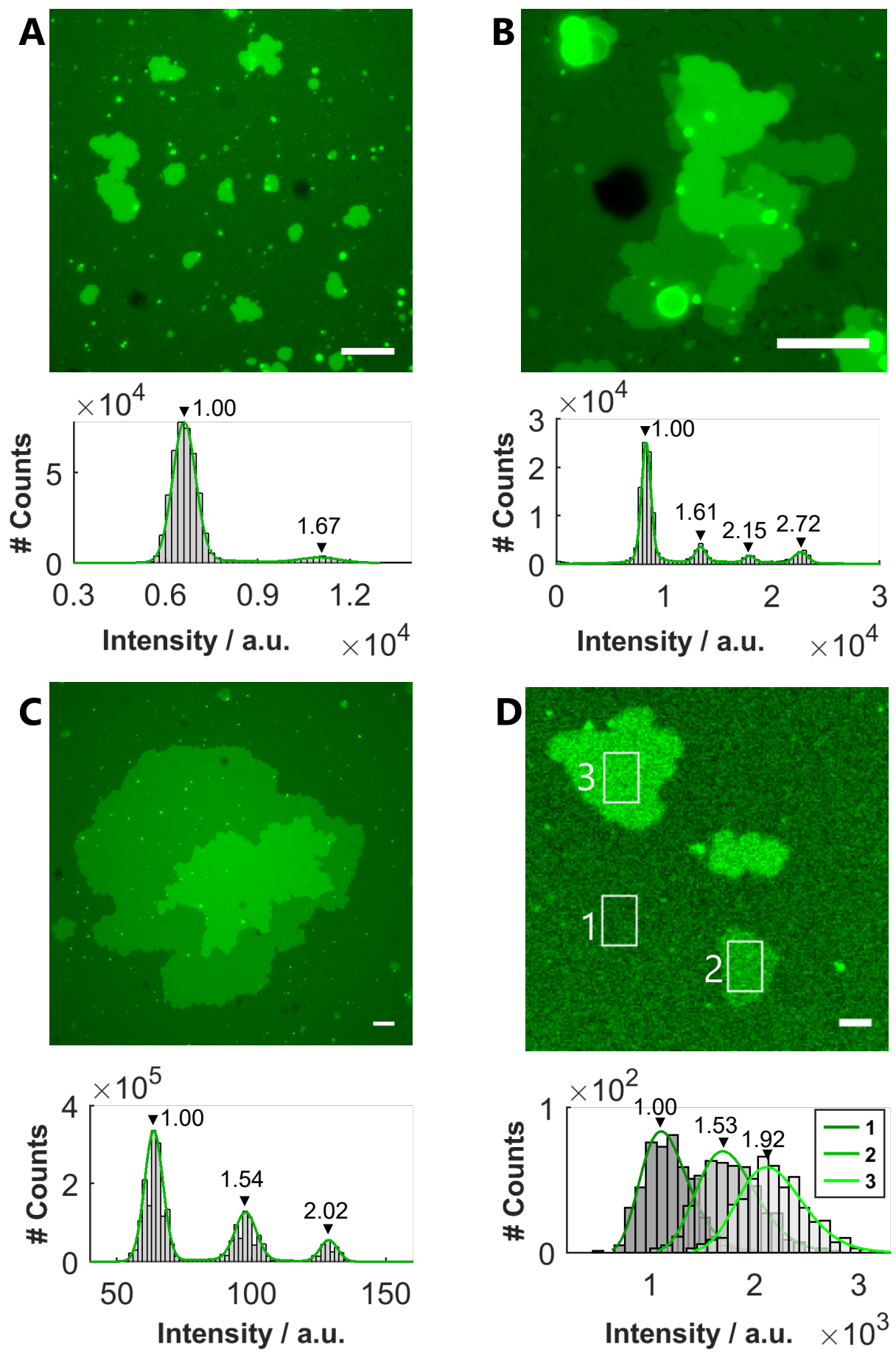

Figure 4.15 Exemplary fluorescence micrographs of brighter patches occurring on POPC:POPG:BP 49.5:49.5:1 membranes under influence of $R_{6}$. Under each micrograph, a histogram with fitted Gaussian distributions of the intensities of all pixels in the image is presented. Relative intensities of the peaks are indicated in the histograms, with the intensity corresponding to the main bilayer normalised to 1 . In $\mathbf{D}$, only pixel intensities within the indicated rectangles were plotted as separate histograms, and to each histogram, a Gaussian distribution was fitted. All scale bars $10 \mu \mathrm{m}$. 


\subsection{Discussion physical effects of R-derivatives}

\subsubsection{Effects of $R_{\text {Side }}$ and $R_{2}$}

Two key results are shared by molecular dynamics simulations and experiments. Firstly, both simulations and experiments showed that properties of POPGcontaining membranes were most strongly influenced by R-derivatives. In simulations, this was shown by a reduction of the kink in the PMF and an accompanied lowering of the pore nucleation energy for POPG. Also, the area per lipid for POPG was increased for R-derivatives, but not in the other lipids. Furthermore, all R-derivatives showed a clear preference for localisation around the PG headgroups, while they did not show any such preference for PC. In the experiments, significant breakthrough force reduction in POPG-containing membranes was found for the small $R$-derivatives $R_{\text {side }}$ and $R_{2}$, which is intuitively relatable to increased likelihood of pore formation presented by simulations.

The second key common finding in simulations and experiments, is that $\mathrm{R}_{\text {side }}$ had the most pronounced effect of all R-derivatives. In simulations, aforementioned parameters showed the strongest alteration for $\mathrm{R}_{\text {side, }}$ while in experiments, breakthrough force was reduced more strongly by $\mathrm{R}_{\text {side }}(\sim 40 \%)$ than by $\mathrm{R}_{2}(\sim 20 \%)$. Furthermore, Rside showed some non-permanent alterations to fluorescence in POPC and POPE-mixed membranes (non-fluorescent areas that disappear again), but in POPG-containing membranes, these changes were permanent (persistent non-fluorescent areas as well as vesicles on the surface).

Two models for possible mechanistic explanations for the $R_{\text {side-induced }}$ fluorescence alterations are presented in Figure 4.16. In Model 1, R local phase separation of lipid bilayers by increasing lipid-to-lipid attraction by bridging neighbouring phosphate groups, and this local gel phase is devoid of Bodipy dye. Besides that, the positive charge of the guanidine group in $\mathrm{R}_{\text {side }}$ screens the negative charge of the POPG lipids. Thus, vesicles from the solution above the membrane settle more closely to the surface or even on the surface, just like for POPC and the POPE-mixture. 


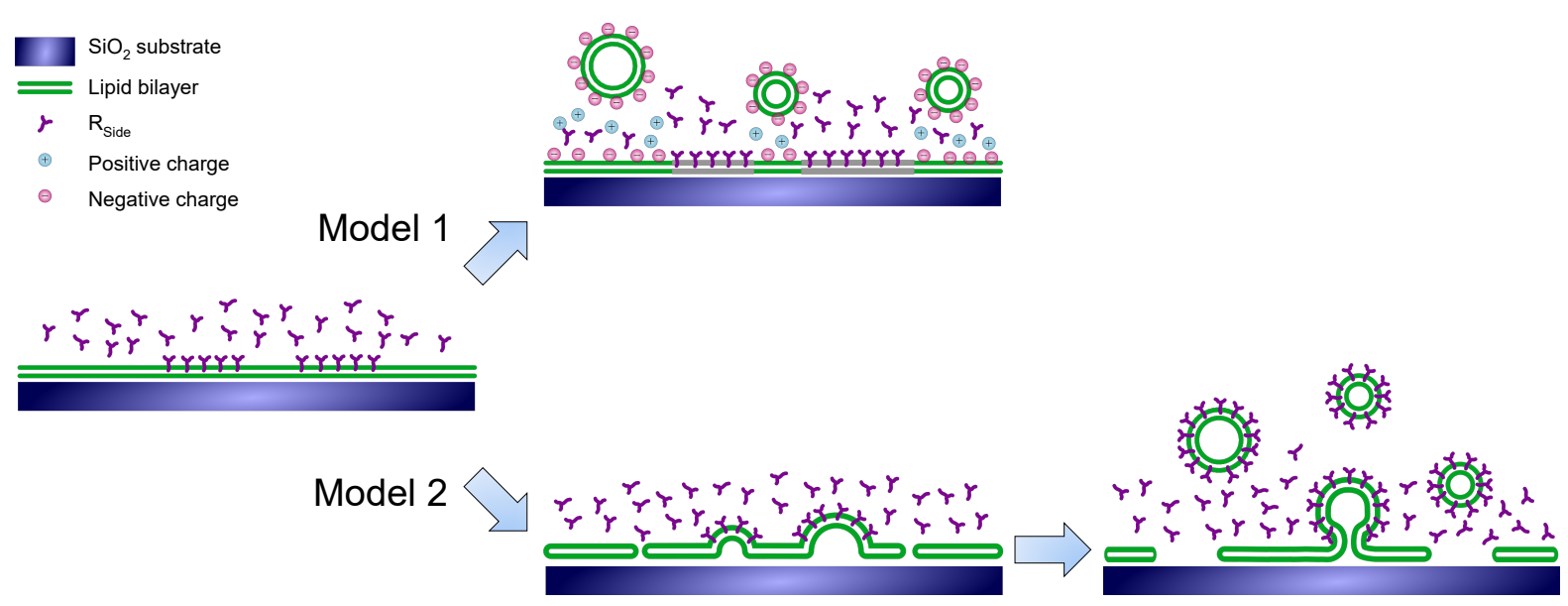

Figure 4.16: Two models to explain the fluorescence observations for $\mathrm{R}_{\text {side }}$ on lipid bilayers. Model 1: $R_{\text {side }}$ causes local phase separation in the planar membrane by facilitating tighter lipid packing, and these tightly packed domains are devoid of dye. The observation of vesicles or round holes on PG-containing membranes is due to Rside's positive charge, which screens the negative charge of the lipids and hence allows for vesicles from solution to settle on or near the bilayer surface. Model 2: Rside acts in a detergentlike fashion, destabilising the bilayer upon binding and increasing the area per lipid. This promotes increased membrane curvature, and in turn, vesicle budding and general removal of material from the bilayer. The domains devoid of dye are actual holes in the bilayer, and the darker domains are caused by area per lipid enlargement by $R_{\text {side }}$ and thus local dilution of dye. $R_{\text {side }}$ and bilayer are not realistically scaled.

This model fits the observed strongest interaction with POPG headgroups, because PG-containing membranes show permanent dark domains as well as still fluorescent but darkened domains. It would also suit best the anecdotal observation for a POPC membrane, of the dark domains that disappear after some time: At first, when the system is not homogeneous, gel-phase domains would be formed, presumably devoid of dye (literature has shown that a similar dye prefers the liquid disordered phase[165]). However, since Rside does not prefer the PC headgroups over the water matrix according to simulations, it partially dissociates again when an equilibrium state in the system is reached. Due to lessened presence of $\mathrm{R}_{\text {side }}$ on the membrane surface, the dark domains dissolve again.

There are however several observations in this work, as well as in literature, that are problematic with this model. The first is that POPG has a phase transition temperature that is the same for POPC, but lower than that of POPE[166]. Therefore, an observation of permanent phase separation in POPG-containing membranes rather than the other two, is difficult to reconcile. Secondly, the deuterium order parameter derived from MD simulations (data not shown), seems to decrease rather than increase for POPG with $R_{\text {side. }}$ This rather points to increased membrane fluidity than to gel phase formation[153], which is not evident from 
either simulation or FRAP data. Thirdly, it is also difficult to reconcile that Rside would cover all of the membrane surface to effectively attract vesicles from solution, but then only cause sparsely divided gel phase domains. Lastly, formation of a gel phase should cause breakthrough force to increase considerably[104], while the opposite was observed upon Rside addition.

The second model (Model 2 in Figure 4.16) comprises detergent-like behaviour by $R_{\text {side: }}$ Rside binds to lipid headgroups and inserts its propyl group between the lipid tails. Thereby, it increases the area per lipid of the solution-facing membrane leaflet and thus promotes positive membrane curvature. Therefore, the apparent vesicles and small round holes observed by fluorescence could be interpreted as vesicle budding. These vesicle-like structures are too large to be SUVs from solution, which makes it most likely that they stem from vesicle budding. By vesicle budding, lipid material is removed from the membrane, and therefore holes appear in the form of the observed dark areas.

This detergent-like model fits all data that imply destabilisation of the bilayer, and they are numerous: first the observation of lowered pore nucleation energy in simulations, combined with reduced breakthrough force. The simulation's indications of membrane thinning and area per lipid increase, and hence more disorder, fit this model as well. The RIfS experiment showing loss of the lipid bilayer when the concentration of Rside was increased further, provides convincing evidence that detergent-like behaviour is closest to the truth.

The temporary alterations to POPE-containing membranes or 'pure' POPC are less directly relatable to this model. They could however be explained when assuming that the observations show a non-equilibrium state of the sample. Analogous to simulations, in equilibrium $R_{\text {side }}$ does not prefer binding to these membranes, and hence will not show alterations there when the system reached homogeneity. In case of the POPE-mixture, beginning vesicle budding could be observed, and hence also tears in the membrane in the form of larger holes. This process does not continue in the forward direction (complete vesicle budding and dissociation and enlargement of holes), but instead Rside partially dissociates again and the system reverts back to its original state.

Only the anecdotal fluorescence observations for POPC and the darker but still fluorescent areas for POPG are more difficult to explain by this model; instantly appearing larger holes in the POPC membrane and re-spreading of vesicles into 
them is unlikely in case of a detergent. Otherwise, the detergent model suits all other experimental data, as well as literature observations. Therefore, we consider Model 2 the most reliable explanation of the fluorescence microscopy findings for $\mathrm{R}_{\text {Side }}$ influence on lipid bilayers.

Besides the overall good agreement, three differences could be found when comparing the MD simulations with the experimental results in this work. Firstly, the cumulative effect of $\mathrm{R}_{\text {side }}$ was not observed experimentally: doubling the concentration did not result in a more pronounced breakthrough force reduction. Secondly, simulations showed an effect of capped $R_{2}$ on POPG, but not for the fully charged peptide as it was used in experiments. While in experiments, breakthrough force was slightly reduced by $R_{2}$.

Thirdly, membrane thinning by R-derivatives was observed for POPG in simulations. Before conducting experiments, this was postulated as the main effect of $\mathrm{R}_{\text {Side, }}$ instead of compromising membrane integrity. Experimental data point to the opposite: no difference can be observed in breakthrough distance, but the breakthrough force effects, the fluorescence micrographs, and the RIfS measurement, all suggest that membrane integrity is compromised.

The other observed differences between experiments and simulations, such as the cumulative effect of $\mathrm{R}_{\text {side }}$ that is not observed in experiments, could be attributed to the four clear differences between the simulation and the experimental system. The first is the use of lipid mixtures in the experiments rather than pure lipid, which could possibly already account for the absence of a cumulative effect for $\mathrm{R}_{\text {Side }}$ : if $\mathrm{R}_{\text {Side }}$ interacts mostly with $\mathrm{PG}$, effects could be more pronounced on a membrane consisting of pure POPG. The second difference is a free-standing bilayer in simulations, while experimentally, a solid-supported bilayer system was used. The latter adds attractive electrostatic interactions between lipid headgroups and solid support as a stabilising factor into the system[74]. Parallel to this comes the third difference: mechanical stress to cause rupture is induced on both leaflets simultaneously in simulations, and mostly from one side in case of experiments. Fourthly, the way this stress is induced differs: in simulations, only water molecules are artificially forced through the membrane, while in breakthrough force experiments, a silicon nitride cantilever tip is forced near the membrane, and has to overcome an electrostatic and a hydration barrier[167]. 
Overall, experimental and simulation data show more commonalities than discrepancies. This can also be said for the experimental data amongst themselves: there are no clear contradictions between experiments.

\subsubsection{Effects of $R_{6}$}

One branch of results was not yet discussed and will be covered here: results for the arginine oligopeptide $R_{6}$. $R_{6}$ shows no apparent compromising behaviour of membrane integrity, as per breakthrough force results, leakage assays and fluorescence microscopy.

This stands in contrast to the recent model system finding for the slightly longer nonapeptide $R_{9}$ : Leakage assays clearly showed leakage of calcein[11], which is a larger dye than the dyes employed in this work. These findings even led to a proposal for a new oligo-arginine cell-penetrating peptide mechanism of entry: causing the membrane to self-fuse, and consequently entering the vesicles or cells through the opened fusion pores[11] (see also 1.3.2 Cell-entry mechanisms for arginine-rich CPPs, p. 21 ff.).

Given that $R_{6}$ and even $R_{5}$ is considered a cell-penetrating peptide and already used in drug discovery efforts[34, 35], the lack of leakage found in this work would have serious implications for the recently proposed fusion pore-entry mechanism of action[11]: it is unlikely that this mechanism is a generally suitable mechanism for all active oligo-arginines. Mechanisms will be more elaborately discussed in an overarching discussion in Chapter 6 ( $p .137 \mathrm{ff}$.) when all data have been presented.

A further observation made by Allolio et al. was lamellarisation of giant vesicles caused by $\mathrm{R}_{9}[11]$. The more brightly fluorescent patches in PG-containing membranes observed in this work, suggest that this is also the case for $R_{6}$. This will be further evaluated after the more elaborate characterisation of these patches in the next chapter and discussed in detail in Section 5.6 p. $120 \mathrm{ff}$. . 


\section{ChARACTERISATION OF R6-INDUCED DOMAINS}

At the end of the previous chapter, the discovery of more intensely fluorescent patches on POPG-containing membranes was presented. In this chapter, efforts to further analyse and characterise these patches will be evaluated. First, the geometry of the patches will be scrutinised. Then, growth and mobility of domains will be analysed by fluorescence microscopy. After that, the focus will be moved to molecular aspects of patch formation, in terms of what materials are necessary for their formation. In connection with that, dissociation constants of different length R-peptides will be discussed. The chapter will conclude with a discussion of these findings.

\subsection{Geometry of domains}

The previous chapter presented the different levels of fluorescence that the patches on POPG-containing membranes could have, along with the suspicion that this could indicate membrane stacks. Here, data will be presented to investigate the validity of this assumption by characterizing the patches.

Figure 5.1 presents a fluorescence micrograph as well as an AFM image of the same section of a POPG-containing membrane (as mirror image for easy comparison), in the presence of $1 \mu \mathrm{M} \mathrm{R}_{6}$. Gaussian distributions were fitted to the histograms below the images. In both distributions, the peak corresponding to the brighter patches differs twice as much from the main membrane (main peak to the left) as the middle peak. Furthermore, in the AFM height image (Figure 5.1 B), each patch level corresponds to about $8 \mathrm{~nm}$ difference. This seems to be much more than the membrane thickness difference usually associated with phase 
separation $(0.5-1.5 \mathrm{~nm},[156,157,168,169])$ or local adsorption of peptide (see Section 5.5 p. $115 \mathrm{ff}$.), and more characteristic of at least another bilayer stacked on top of the main one [105, 154, 169-171]. Another interesting observation, was that the second height level of domains appeared less stable than the first: upon recording a higher resolution image of the rounded structure at the bottom of Figure 5.1 B, this structure disappeared, while the level 1 structures remained intact (see Appendix 10 p. XXXIII).

A
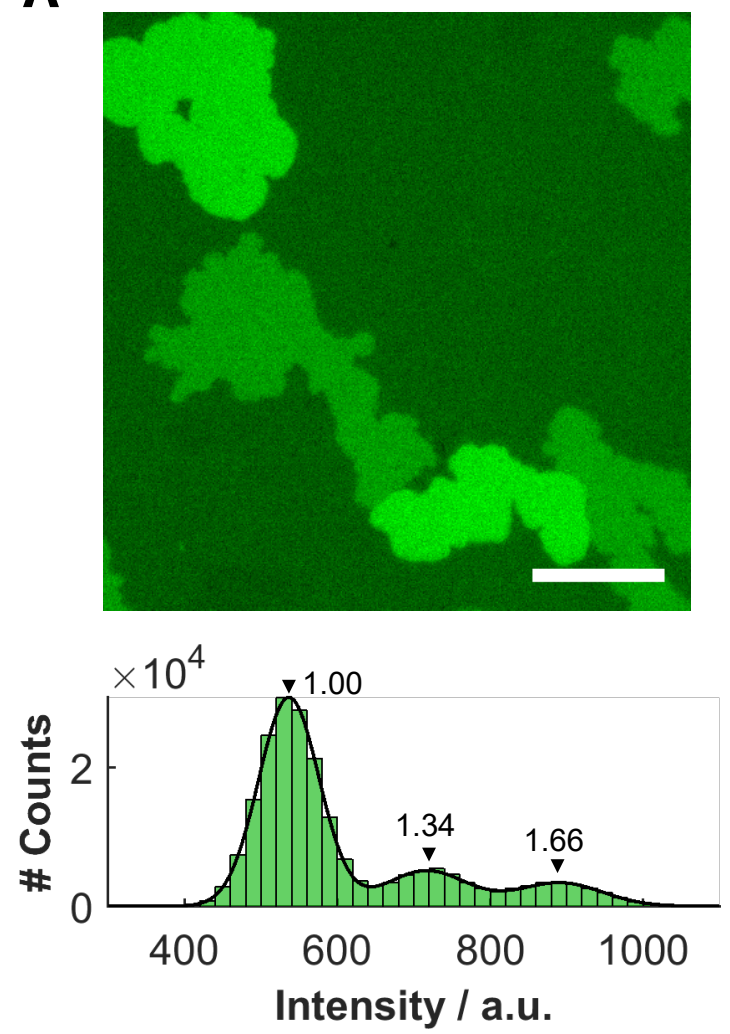

B
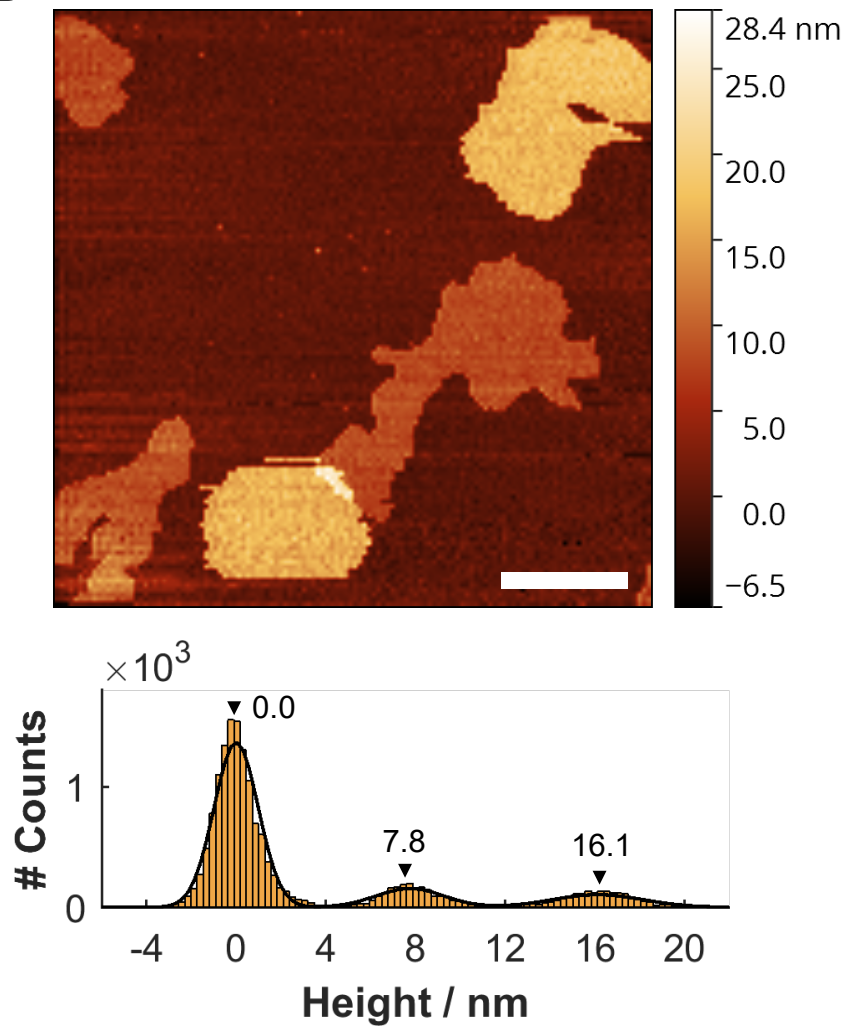

Figure 5.1: Fluorescence micrograph (A) and AFM image (B) of the same section of a POPG-containing membrane in the presence of $1 \mu \mathrm{M} \mathrm{R}$. Below each image, a histogram of measured values is shown, corresponding to fluorescence intensity $(\mathbf{A})$ and measured height with main bilayer normalised to zero (B), respectively. To each histogram, a sum of three Gaussian distributions was fitted (black lines) The means of the fitted distributions are indicated in each histogram. In $\mathbf{A}$, these values were normalised for clarity. Scale bars $10 \mu \mathrm{m}$.

Further indication that observed patches correspond to bilayer stacks was found in a force map at a $1 \mathrm{mM} \mathrm{R} \mathrm{R}_{6}$ concentration (Figure 5.2). In the two images, each pixel corresponds to one force curve, and the values of breakthrough force (A) and breakthrough distance (B) were mapped according to their force curve location. A domain that clearly shows increased breakthrough distance by a factor 2 (B) can be observed, and it also seems to correlate with slightly higher breakthrough force (A). In Figure 5.2 C-F, exemplary force curves are shown, and they are indicated 

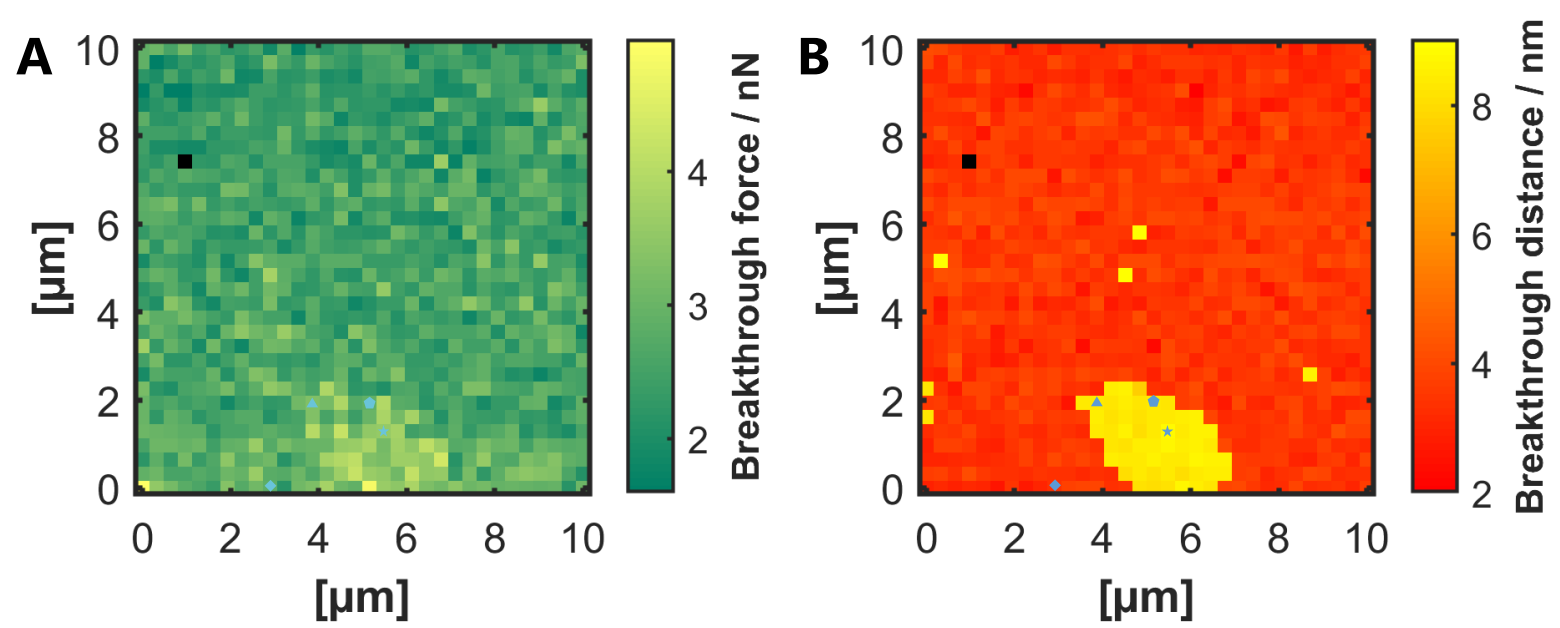

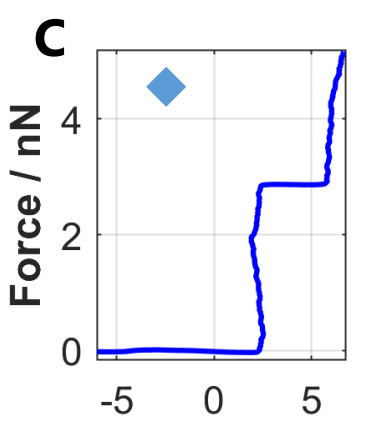

Separation / nm

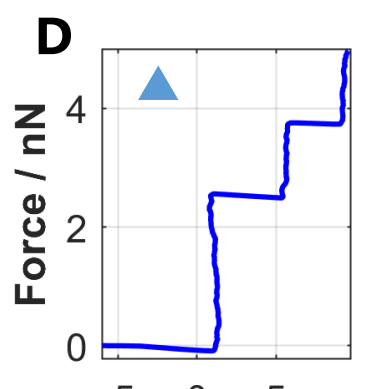

$\begin{array}{lll}-5 & 0 & 5\end{array}$

Separation / nm

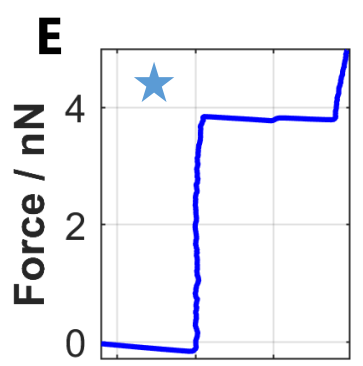

$\begin{array}{lll}-5 & 0 & 5\end{array}$

Separation / nm

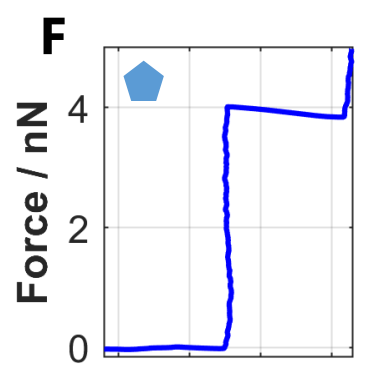

$\begin{array}{llll}-5 & 0 & 5 & 10\end{array}$

Separation / nm

Figure 5.2: Mapped breakthrough force results of a POPC:POPG:BP 49.5:49.5:1 membrane in the presence of $1 \mathrm{mM} \mathrm{R}_{6}$. Each pixel corresponds to one force curve and its deduced breakthrough force (A) or breakthrough distance (B). In aforementioned representations, approach force curves shown in C-F are indicated with blue symbols.

on respective maps in $\mathbf{A}$ and $\mathbf{B}$ by blue symbols. Outside the domain, a typical single breakthrough event is observed $(\mathbf{C})$. Inside the domain, the double breakthrough distance is clearly distinguishable in the force curves. Some force curves showed two clearly distinct events with roughly the same breakthrough distance (D), or two distinguishable events at nearly the same breakthrough force (E). Also, apparently single events(F) with a double breakthrough distance compared to outside the domain were observed.

Aforementioned results were considered enough evidence that observed patches caused by $R_{6}$ addition consist of membrane stacks. Therefore, these phenomena will henceforth be referred to as 'stacks'.

As a next step, fluorescence recovery after photobleaching was employed, to investigate whether observed membrane stacks are attached to the main bilayer and if so, what the nature of this attachment is. Figure 5.3 A and B show that 
both the first and second level stacks (as distinguished by their fluorescence intensity compared to other patches and the main membrane) show recovery of fluorescence after bleaching within a few minutes. This indicates connection with the main bilayer. As far as stacks did not change shape, recovery was uniform and did not appear asymmetrical or otherwise restricted (data in Appendix 10 p. $X X X I I)$, analogous to the observations of Gräb[172]. This indicates, analogously to Gräb's work, that most likely multiple connections to the main bilayer exist.

In some cases, the FRAP experiment caused deformation of the stack. In such cases, fluorescence was only monitored in a fraction of the stack that was stable throughout the FRAP experiment.
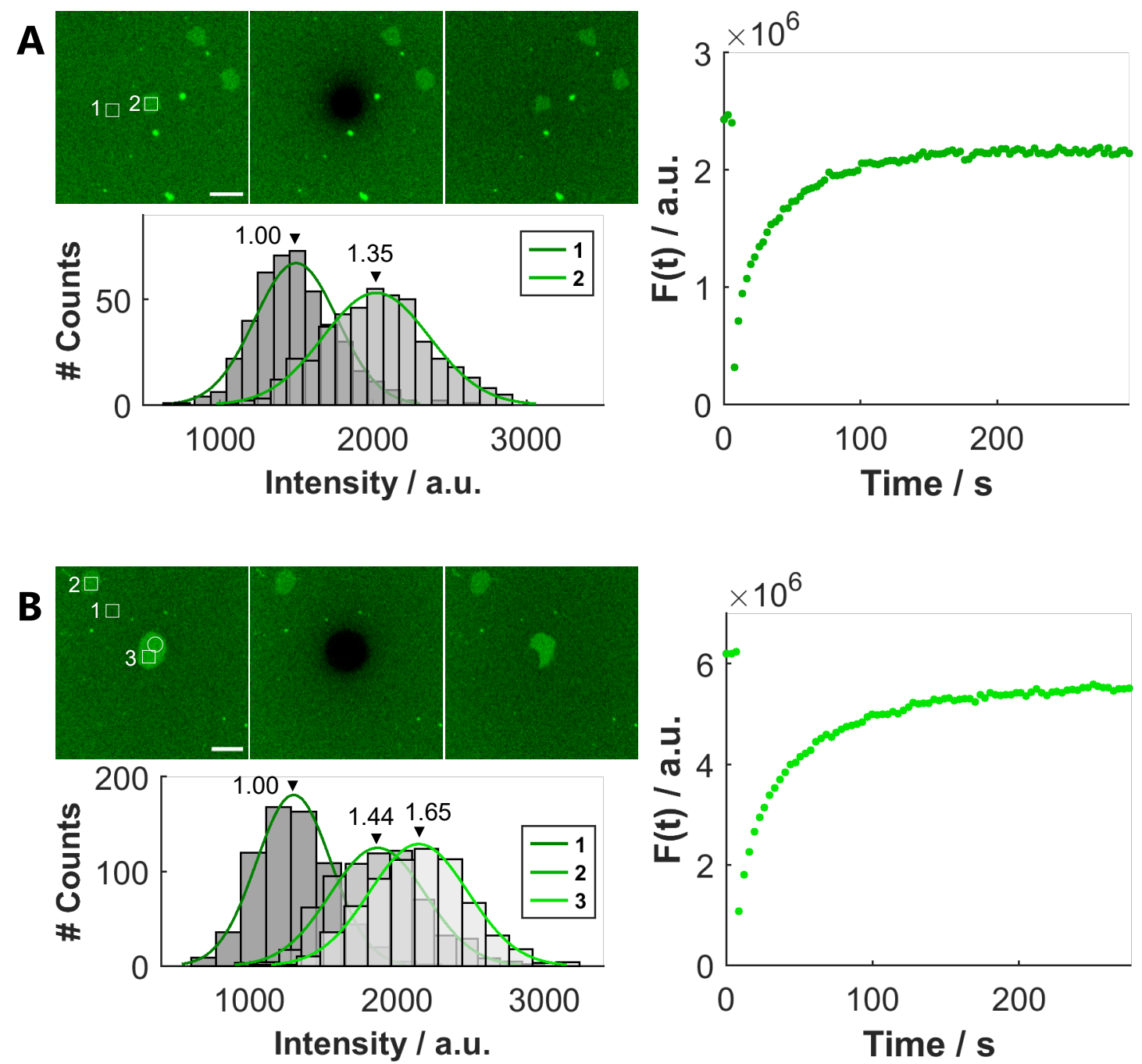

Figure 5.3: Examples of FRAP experiments on membrane stacks of level 1 (A) and level 2 (B). The level of the bleached stack was determined by comparing the integrated fluorescence intensities of regions indicated with white squares, as shown in the histograms below each image series. The fluorescence against time for the bleached region is shown on the right. If the stack changed over the course of the experiment, only a stable fraction of the stack was taken for analysis of fluorescence intensity over time (white circle). Scale bars $20 \mu \mathrm{m}$. 
In order to gain more insight into the nature of the junctions between the stacks and the main bilayer, the mean time constant $T$ for each FRAP experiment was analysed. This method was introduced by Savić et al. in their experiments on fusion pairs of membrane-coated glass beads[91]. It can be obtained by normalising the recovery portion of the FRAP data to 0 after bleaching and to 1 post-recovery (Figure 5.4 A). $T$ is then obtained by integrating the inverse of the normalised FRAP data over time (green region in Figure 5.4 A, right plot). This method provides a means to quantitatively analyse phenomena that cannot be characterised by ordinary lateral diffusion kinetics and a diffusion constant.
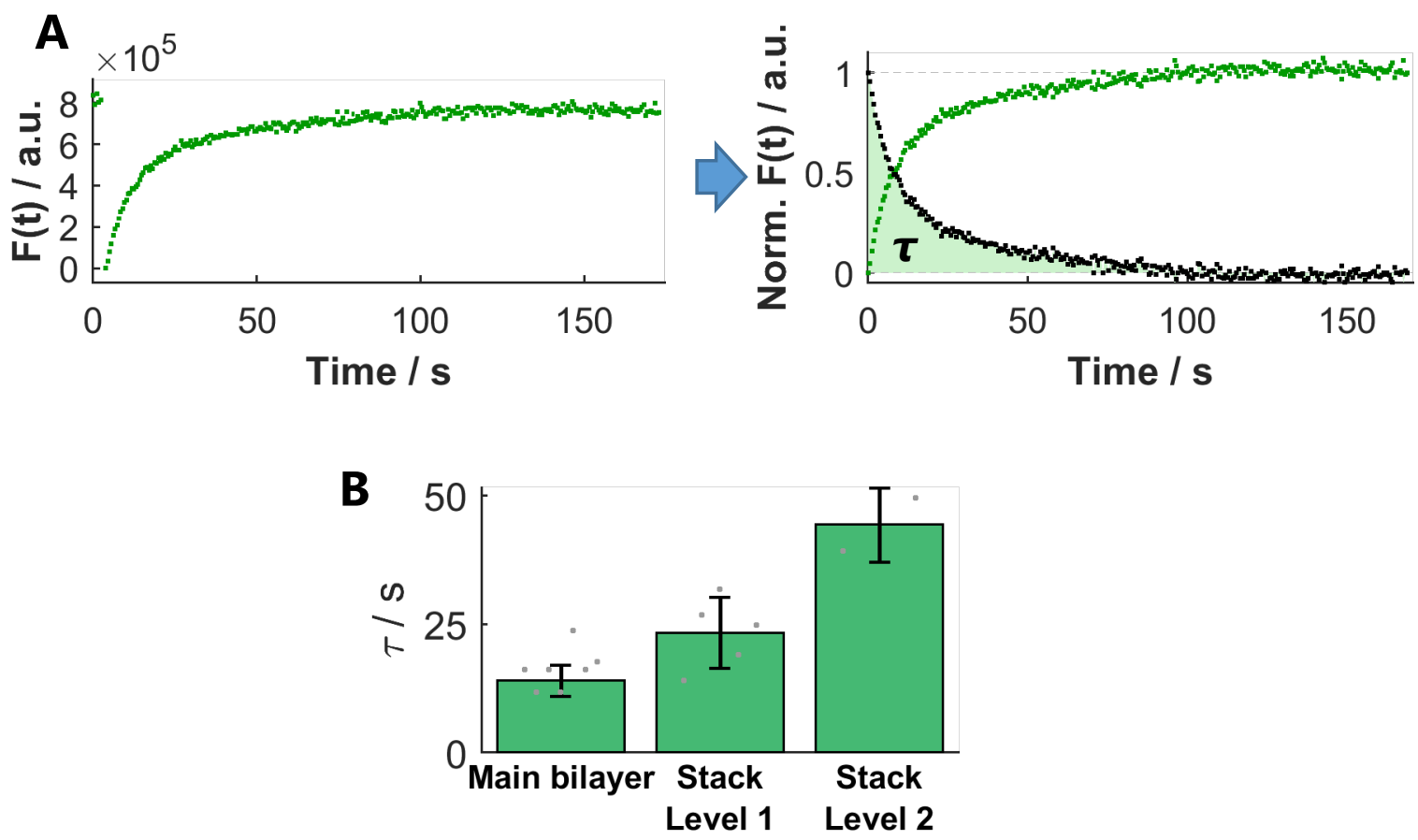

Figure 5.4 A: Illustration of the derivation of the mean time constant $\tau$ (green shaded area) from a FRAP experiment; B: Comparison of the values for $\tau$ of the main bilayer, a level 1 stack and a level 2 stack within one experimental day and with approximately equal bleaching areas. Grey points indicate individual measurements, bars and error bars signify mean and standard error.

In Figure 5.4 B, a plot of $T$-values obtained within one experiment is shown, with bleaching area roughly constant and roughly circular stacks. $T$ seems to differ by roughly a factor 2 when comparing the main bilayer and the first level of stacks, but also when comparing the first and second level stacks. This factor two is reminiscent of the height difference between different levels of stacks introduced earlier (Figure $\mathbf{5 . 1}$ p. 92). It suggests that there might be a relationship between $T$ and the amount of material that needs to diffuse. 


\subsection{Dynamics of stack formation}

Several time-dependent fluorescence observations were made of stack formation or alteration. Firstly, inhomogeneity of BP-fluorescence was often observed briefly after $R_{6}$ addition (Figure 5.5): after about 30 seconds, the fluorescence showed irregular darker and brighter spots, which became larger over the course of minutes. The membrane returned to homogeneity over the course of about seven minutes after R-peptide addition. The micrographs series shown was recorded on a POPS-containing membrane (see Section 5.4.2 Figure 5.9 p. 100), but this behaviour was also typically observed in POPG-containing membranes.

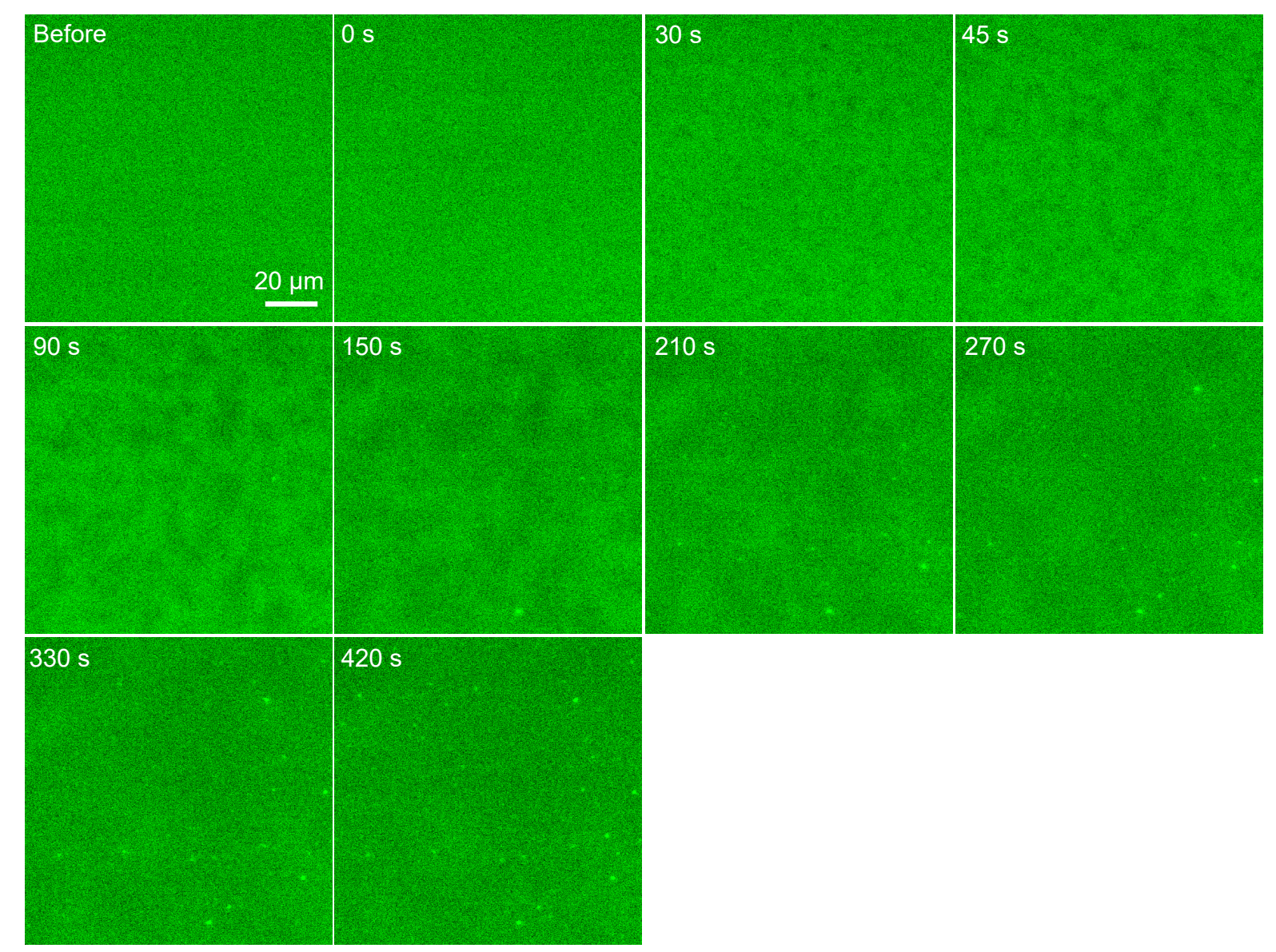

Figure 5.5: Fluorescence micrograph series on a membrane of composition POPC:POPS:BP 49.5:49.5:1, one frame before R-peptide addition and the first seven minutes after R-peptide addition. Elapsed time in seconds is indicated on the top left of each frame.

Secondly, the area size growth of stacks was computed using a combination of particle analysis in Image] and a self-written Matlab script to sort the data. In Figure 5.6, the growth of stacks after R-peptide addition is shown. The outlines 
of the stacks used for area computation are coloured and numbered the same as their corresponding area graphs in Figure 5.6 B. Four smaller stacks fused into two larger stacks (inset in Figure $5.6 \mathrm{~B}$ ). The growth of the larger stacks plateaued after approximately 10 minutes, and slightly shrunk again by the end of the recording. Initial growth rate was about $0.2-0.4 \mu \mathrm{m}^{2} \mathrm{~s}^{-1}$ for the larger stacks, the lower initial rate belonging to stack 6 which came into existence later.

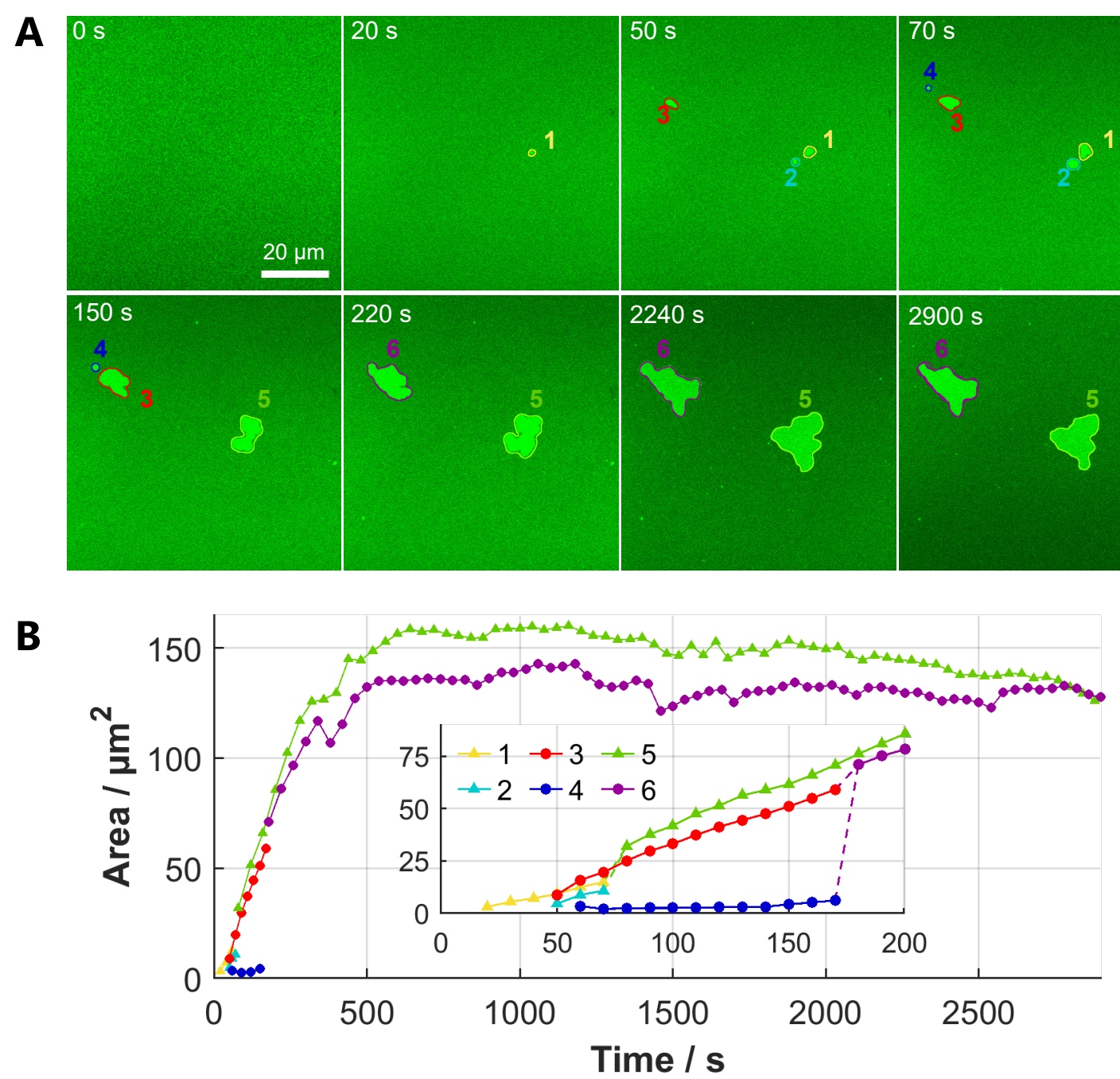

Figure 5.6: Stack growth analysis briefly after $R_{6}$ addition (illumination only when recording images every 10 s). A: Fluorescence micrograph snapshots for several time points, with contours indicating areas used for analysis. Contour colours and numbers correspond to the plots in B. Numbering was assigned in order of formation of the stacks. B: Plot of stack area versus time. Stack pairs that fuse together, as well as their fusion product, are shown with the same symbol. Inset: Fusion of four smaller stacks into two larger ones during the first $\sim 180$ seconds after R-peptide addition.

Thirdly, it was observed that stack growth and formation could also occur under the influence of epifluorescence illumination, more than an hour after R-peptide addition. Results are shown in Figure 5.7, again with outline colour and number corresponding to colour and number in the area growth plots in Figure 5.7 B. 
A
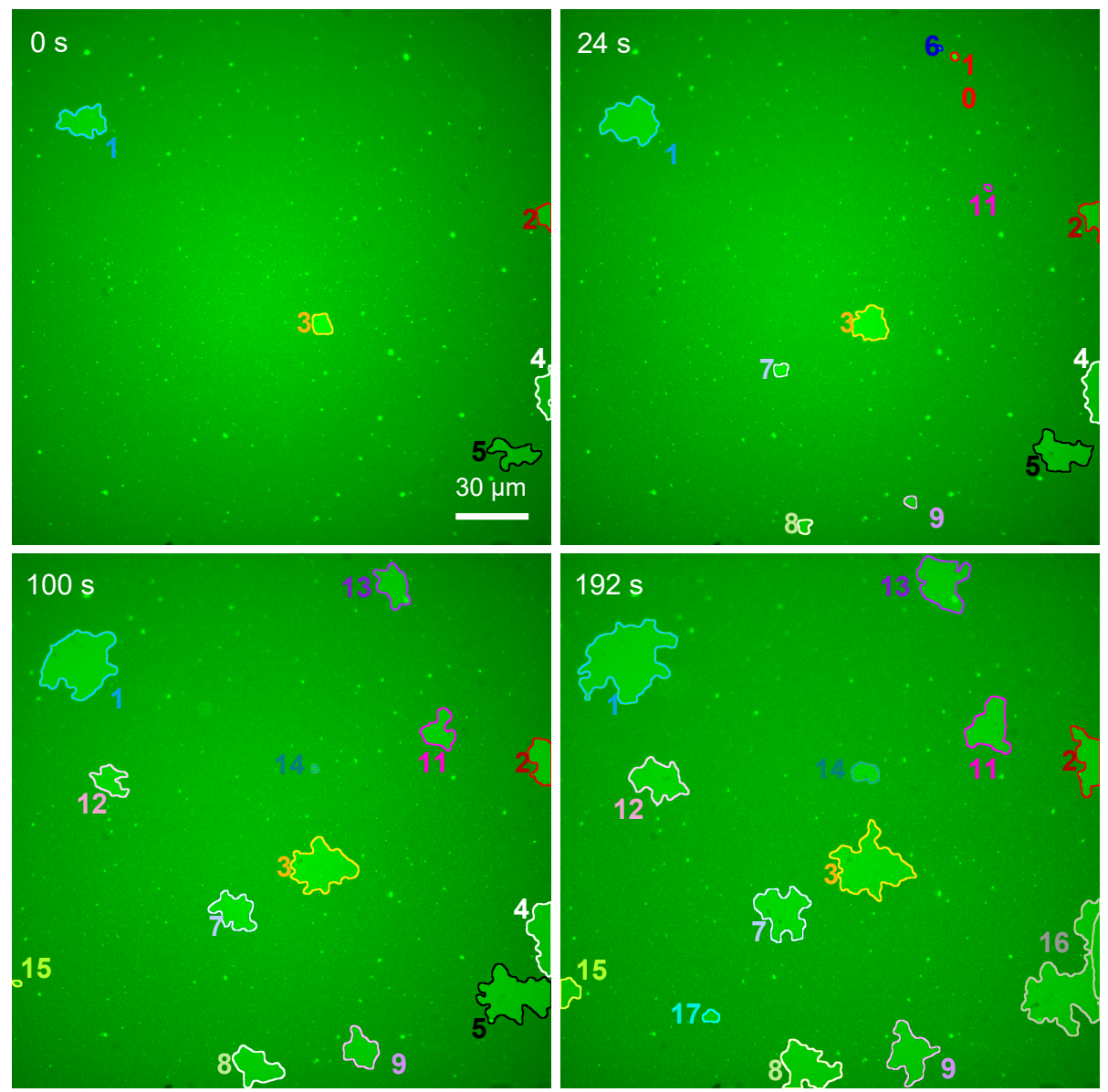

B
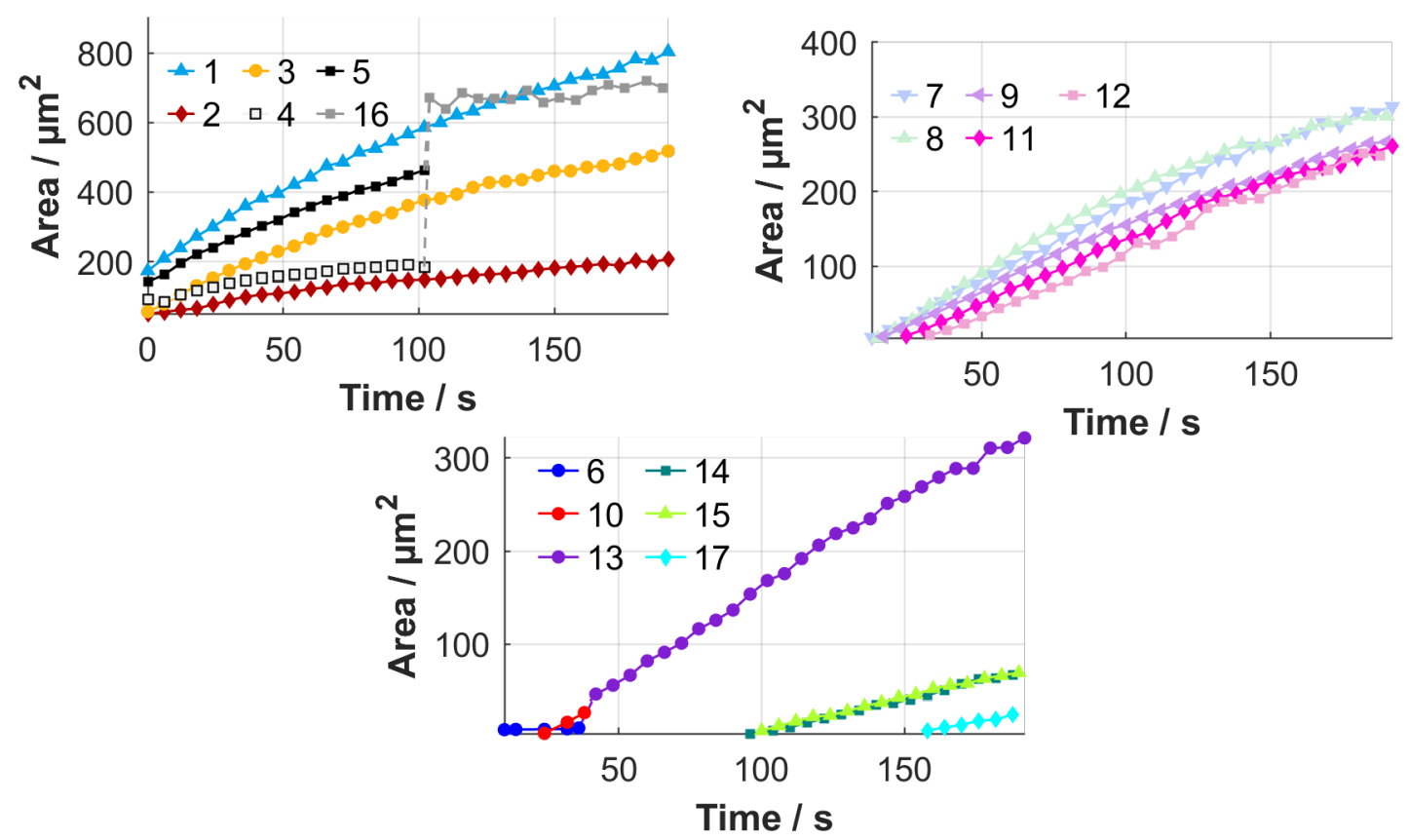

Figure 5.7: Stack area analysis on a POPG-containing membrane, an hour after R-peptide addition, under continuous epifluorescence illumination. A: Fluorescence micrograph snapshots; Contour colours and numbers correspond to area-vs-time plots in B. Numbering was assigned in order of formation of the stacks. B: Area-vs-time plots for the stacks shown in A. Stacks of similar size are plotted separately, for clarity. Stacks that fuse together and their fusion products are plotted with the same symbol. 
Surprisingly, in this case, a limit to stack growth rate and a hint at plateauing could be observed as well, despite the short recording time. Long recording was not feasible because of fluorophore bleaching. The second surprising observation, is that in this case, initial stack growth rate was higher by a factor $10: 1.5-5.0 \mu \mathrm{m}^{2} \mathrm{~s}^{-1}$ for stacks that formed in the first 50 seconds of recording. This was unlikely to be related to the total area, since stack 7, 8 and 9 started with areas comparable to the starting areas in Figure 5.6 and showed initial growth rates of 2-2.5 $\mu \mathrm{m}^{2} \mathrm{~s}^{-1}$ too.

Stacks that formed later also had a lower growth rate, which appeared linear: Stack 14, 15 and 17 showed an initial growth rate of 0.5-0.7 $\mu^{2} \mathrm{~s}^{-1}$, which was similar to the final growth rate of several larger stacks (2, 9 and 18).

Fourthly, we would like to zoom in on a few observations concerning the formation and growth of single stacks. The first is a closer look at Stack 1 from Figure 5.7. By the end of the image series, a vesicle comes into view. Over the course of 10 seconds, it seems to fuse onto the top border of the stack (Figure 5.8). This shows that at least part of the lipid material from the stacks stems from the surrounding solution.

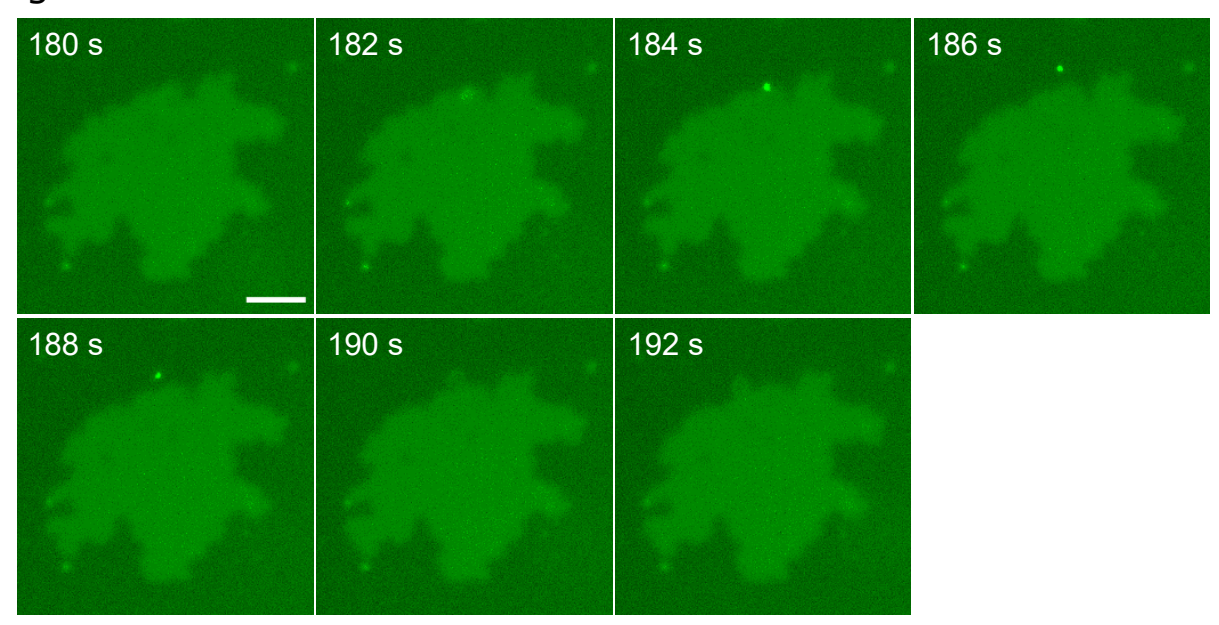

Figure 5.8: Zoomed in fluorescence micrograph series of the last seven frames of Stack 1 also shown in Figure 5.7. A vesicle coming into focus at $182 \mathrm{~s}$, seems to fuse to the stack. Time indicated is time since R-peptide addition. Scalebar $10 \mu \mathrm{m}$.

The second observation, is an apparent overlap between stack behaviour and tube formation in POPS- or POPG-containing membranes without R-peptide. In Figure 5.9 A, some of the last snapshots after R-peptide addition are shown for a stack on a POPS-containing membrane. The stack finishes growing to a maximum, then shrinks again, and by the end of the recording, apparently membrane tubes extend from its border. At the same time, the vesicles present 
on the membrane have fused into the main membrane. Figure 5.9 B shows that the opposite was also possible: during recovery from a FRAP experiment (bleaching event not shown), T-shaped stack was surrounded by four tube-like structures (numbered in each image for clarity). During the last part of the recovery recording, two of these structures ( 2 and 4 ) shrink and apparently thereby cause extension of the stack, while one moves down but seems to keep its shape (3). Structure 1's material seems to have split off at the end, and to drift away in in

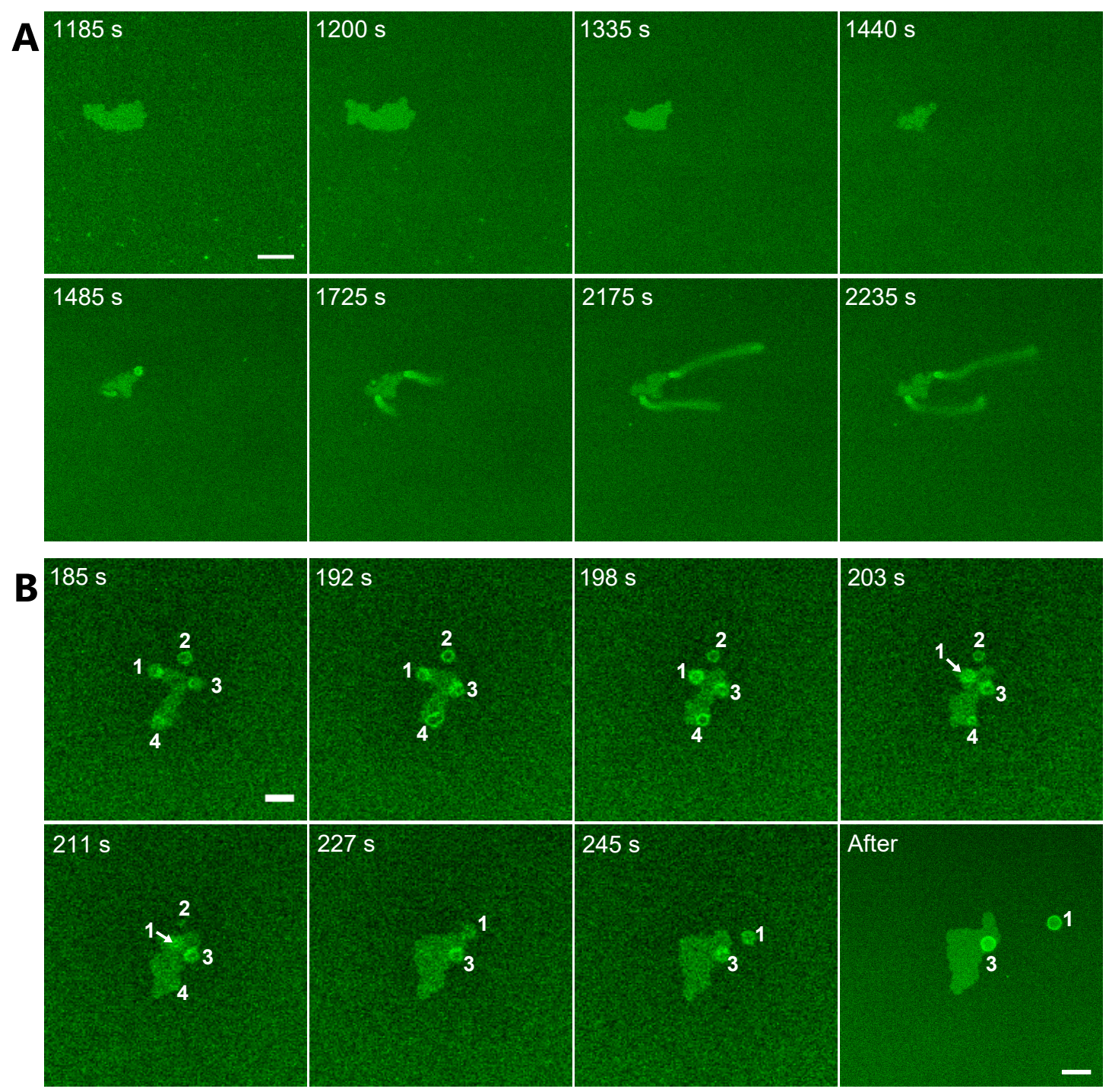

Figure 5.9: Two fluorescence micrograph series on POPC:POPS:BP 49.5:49.5:1 membranes, illustrating dynamic behaviour of stack growth and shrinkage in relationship to tether formation and vesicle budding. A: Last part of stack growth after R-peptide addition; after reaching its maximum size, the stack shrinks again and develops tube-like protrusions from its borders. At the same time, vesicles on the bilayer around it acutely disappeared, presumably fusing into the main bilayer. Time on the top left indicated in seconds after R-peptide addition. B: A T-shaped stack with four tube-like protrusions around it, numbered 1-4. While structure 2 and 4 fuse with the stack, and 3 only moves down, Structure 1 splits off in the form of a vesicle and drifts away. The latter is clearly visible in the higher resolution image taken after the FRAP experiment. Time on the top left indicated in seconds after bleaching. Last image was taken after FRAP measurement with a higher resolution. All scale bars $20 \mu \mathrm{m}$. 
the right-hand direction in the form of a larger vesicle, as is clearly visible in the image taken after the FRAP series.

It should be noted that while here, mostly images on POPS-containing membranes are shown as examples, similar behaviour was also observed in POPG-containing membranes and recorded as individual images rather than series. An example can be found in Appendix 11 p. XXXIV. 


\subsection{Origin of lipid material}

Previously shown results show clear indications of lipid material exchange between the stacks and the surrounding solution. However, this provides no direct proof of how stacks form initially, and what provides the lipid material for their initial formation: is there an excess of lipids within the continuous membrane, or is the material directly extracted from vesicles in the solution? This subchapter presents three experimental approaches to address this question.

\subsubsection{Addition of vesicles with different dye}

The first approach was one also employed by Gräb et al.[170, 172]: adding a vesicle population bearing a different dye to a previously prepared and thoroughly rinsed solid-supported lipid bilayer, prior to adding the stack-inducing agent. If the second dye only or mainly shows up in stacks, that would be evidence of direct fusion of vesicles on the supported membrane.

First, vesicles containing 1\% Texas Red were added to a membrane containing the previously used green Bodipy lipid tail dye (Figure 5.10 A-E). An image of the observed result is presented in Figure 5.10 A, with accompanying histograms of the intensities of both dyes in both main membrane and stacks in B. It has to be noted that Texas Red fluorescence was strongly artificially enhanced in order to be visible, during recording as well as during processing. Nevertheless, in most recordings, Texas Red showed higher intensity in the stacks, by a stronger factor than Bodipy: Where for Bodipy the factor is 1.4-1.7, Texas Red seems to show a factor 2 . This factor 2 was also consistently observed by Gräb et al. for stacks on membranes with Texas Red as dye[170].

On the Bodipy-rich stacks in Figure 5.10 A, several round structures with intense Texas Red fluorescence were observed, which seemed too large to be plain vesicles. An exemplary FRAP experiment on such a structure, wherein both dyes were bleached, is shown in Figure $\mathbf{5 . 1 0} \mathbf{C}$ and D. From the images in Figure 5.10 C, it is apparent that the structure showed no recovery of Texas Red, but instead, filled with Bodipy. The normalised fluorescence intensity of the bleached spot shown in Figure 5.10 B did show recovery for Texas Red, but by less than $50 \%$. This shows that there definitely is fusion of Texas Red-containing 
vesicles with the main membrane, but Texas Red did not seem to diffuse between membrane stack levels. The partial recovery that was observed was likely due to diffusion from the rest of the larger stack below the small structure that was bleached. Most similar red structures on the visible stacks in Figure 5.10 A behaved in the same way when bleached, except that one structure did not show any recovery for either dye (data not shown).
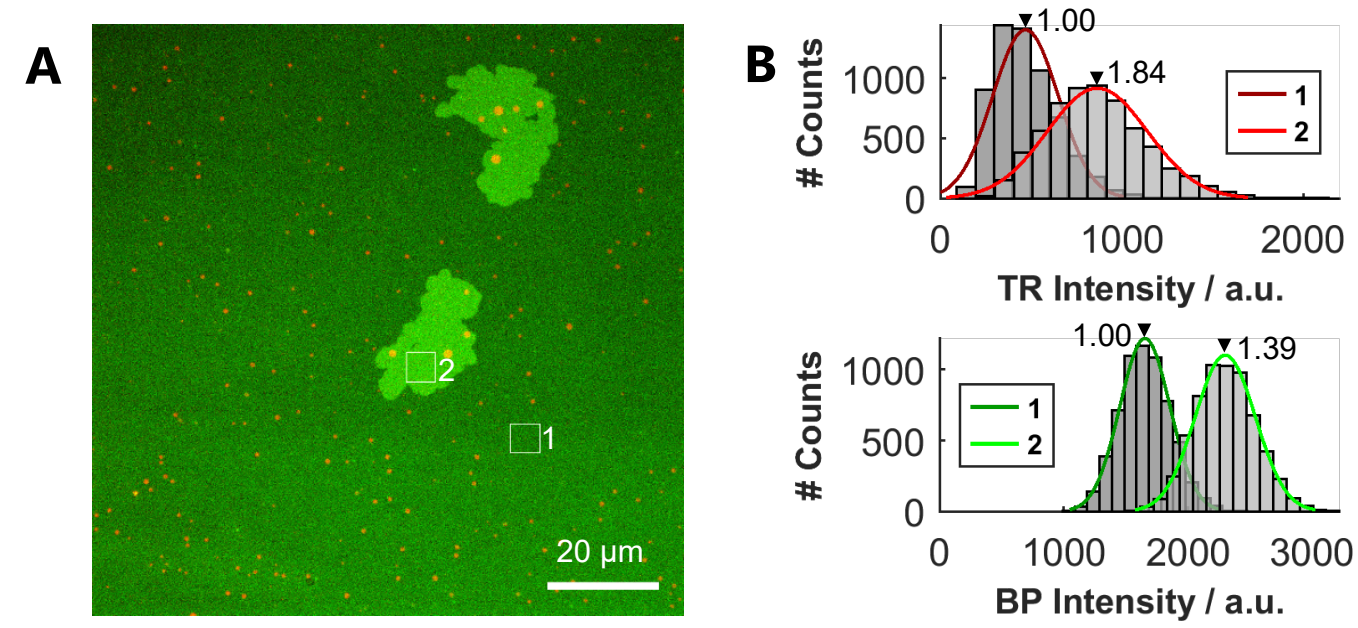

C
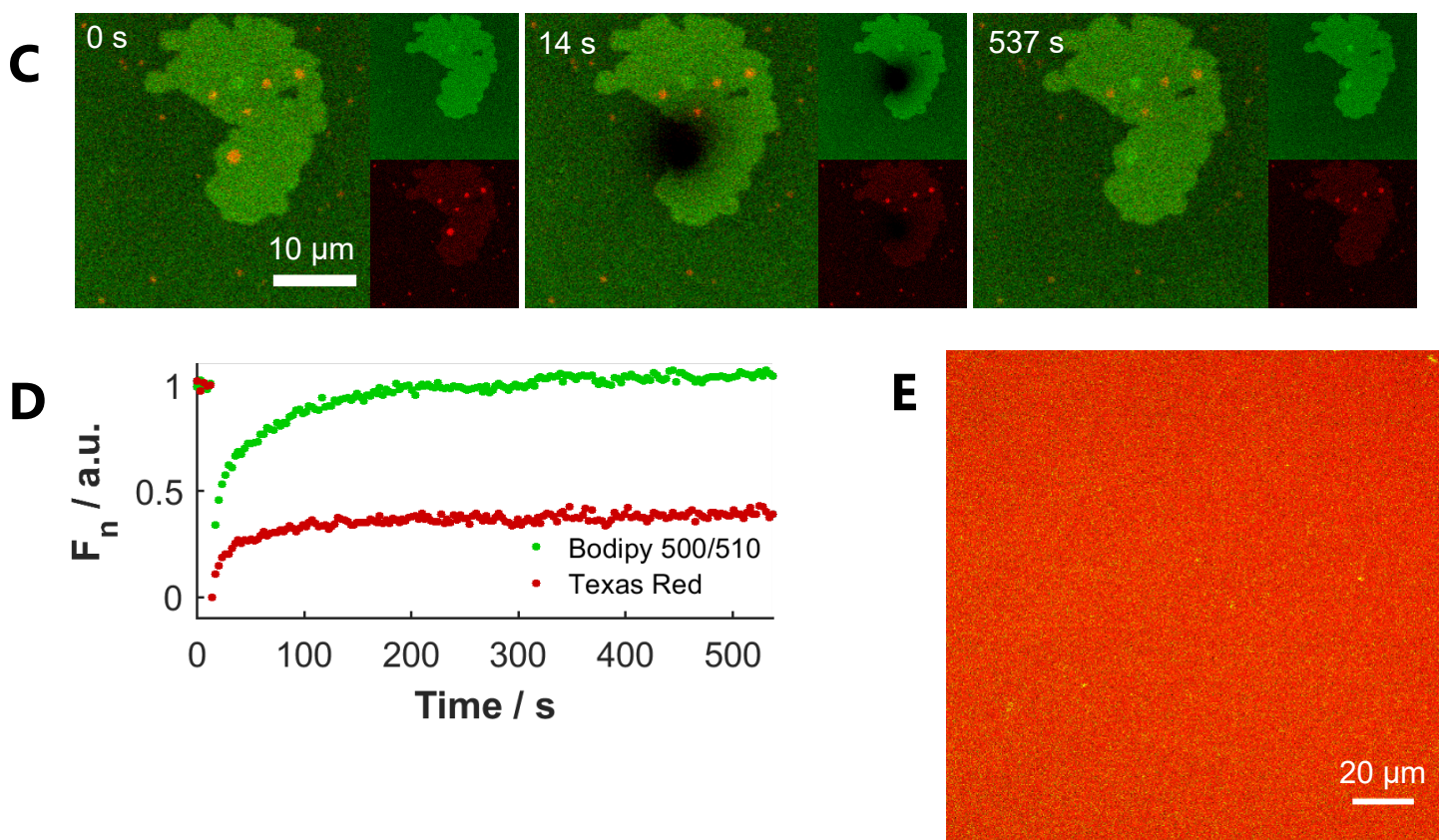

Figure 5.10: Results for a POPC:POPG 1:1 membrane with an added vesicle population with a different dye, and subsequent addition of $1 \mu \mathrm{m} \mathrm{R}_{6}$. A: Exemplary image of a bilayer containing $1 \%$ Bodipy, after addition of $1 \%$ Texas Red-containing vesicles and $R_{6}$. B: Histograms of pixel intensities of the square regions indicated in $\mathbf{A}$, for Texas Red (upper plot) and the green Bodipy (lower plot). Relative intensities are indicated at the peaks of the fitted Gaussian distributions. C: FRAP experiment on a small circular suspected stack, both dyes were bleached. Images from left to right before bleaching, after bleaching, and after recovery. Time indicated on the top left is recording time in seconds. D: Fluorescence intensity of the bleached spot of the experiment in $C$, normalised to 1 for values before bleaching, and to 0 for the first point after bleaching. E: Exemplary image of a bilayer containing Texas Red, after addition of Bodipy-containing vesicles and $R_{6}$. 
Figure 5.10 E presents an exemplary fluorescence micrograph of the same experiment with the dyes exchanged: Texas Red in the solid supported membrane, while Bodipy-containing vesicles were added before $\mathrm{R}_{6}$-addition. No stacks were observed in this case, although the membrane showed some green dye presence and there was clear adhesion of vesicles from solution (green spots).

\subsubsection{RIfS with vesicles present}

To explore the importance for vesicles for stack formation in a bulk fashion, as well as to access membrane behaviour without dye present, RIfS experiments were performed. In these experiments, the rinsing after membrane formation was performed a closed system fashion. 'Closed' is defined as follows: $1.2 \mathrm{~mL}$ of measuring buffer (ca. twice the system volume) was used as the inlet as well as the outlet for the system. This measuring buffer was exchanged five times, effectively achieving an amount of $0.002 \mathrm{mg} / \mathrm{mL}$ of lipids still in the system, which should be similar to the amount that was always present in the samples for atomic

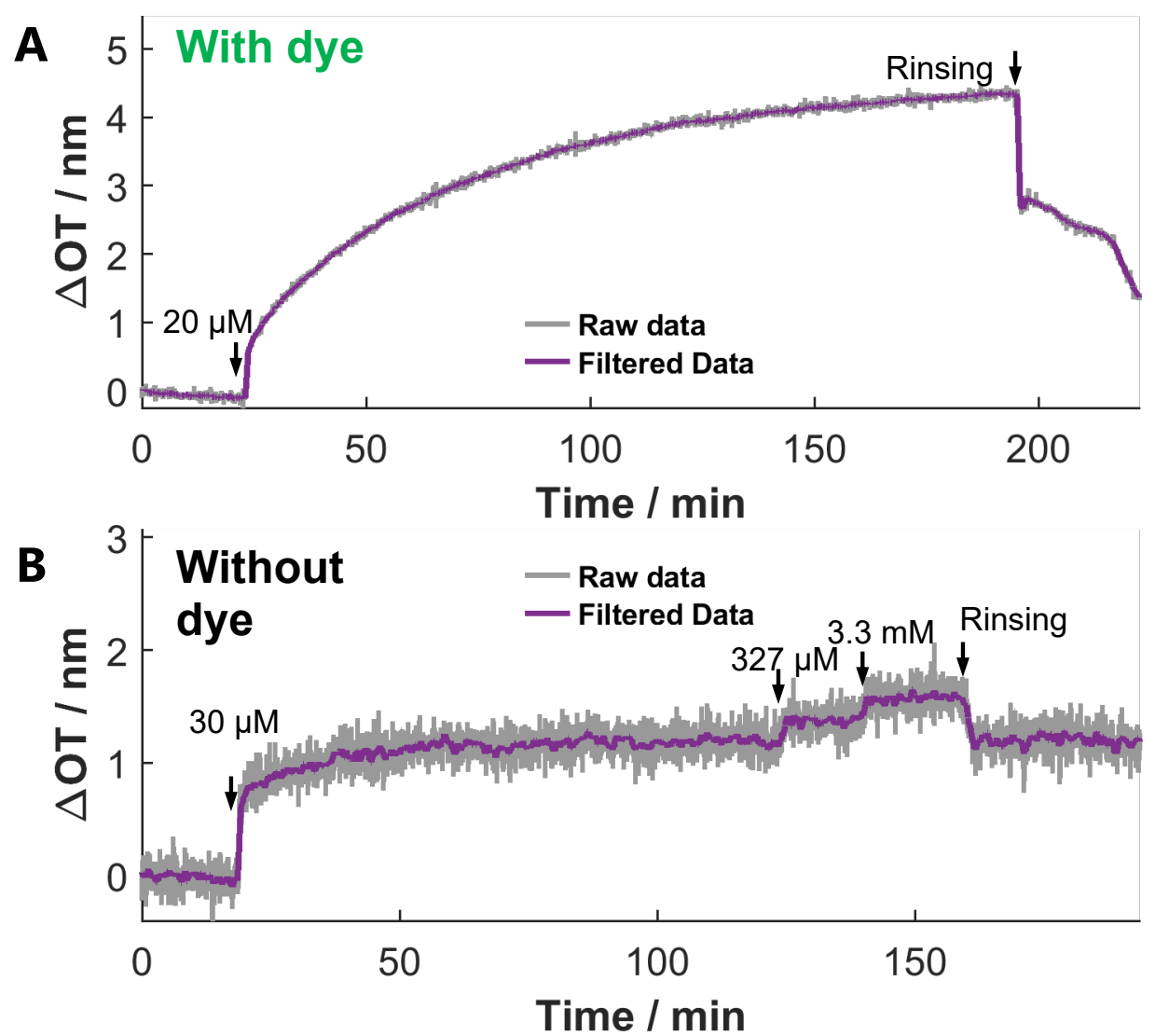

Figure 5.11: Closed system RIfS experiments with $R_{6}$ addition to POPC:POPG 1:1 bilayers. Optical thickness is shown after bilayer formation and closed system rinsing. $R_{6}$ concentrations as well as a afterwards rinsing are indicated in each figure. A: $1 \%$ Bodipy dye, which was also used in AFM and microscopy experiments; $\mathbf{B}$ : Without dye. 
force microscopy and fluorescence microscopy (see 3 Materials \& Methods pp. 50-52 for details).

After this closed system rinsing, $\mathrm{R}_{6}$ was added in a concentration of 20 or $30 \mu \mathrm{M}$, since this was observed to lead to saturation of the system in dissociation constant determination (see Section 5.5 p. 115 ff. for results). In Figure 5.11, the optical thickness change in 50 \% POPG membranes is shown after membrane formation and rinsing, for a membrane with the usual 1 \% green Bodipy dye, and for a membrane without dye. In case of the membrane with dye (Figure 5.11 A), $4 \mathrm{~nm}$ increase in optical thickness was observed over the course of 2 hours. This behaviour was reproducible (data not shown). It was not observed to this extent without dye (Figure 5.11 B) or in 50 \% POPS membranes (data in Appendix 8 p. $X X X I$, less extreme OT increase); in these other experiments, the maximum optical thickness increase was about $2 \mathrm{~nm}$.

Another observation from this experiment, is that this optical thickness increase was at least partially reversible (Figure 5.11 A right hand side). Especially later added $\mathrm{R}_{6}$ in higher concentrations washed off immediately (see also Appendix 8 p. $X X X I)$.

\subsubsection{Membrane patches on PDMS}

In order to investigate the role of a continuous membrane for stack formation, a non-continuous bilayer was formed on PDMS (see Materials \& Methods p. 51). The used method produced small membrane patches of $2 \mu \mathrm{m}$ diameter, with otherwise the same conditions for producing a solid supported lipid bilayer. $1 \mu \mathrm{M}$ of $\mathrm{R}_{6}$ was added as before, and increased to $10 \mu \mathrm{M}$.

A

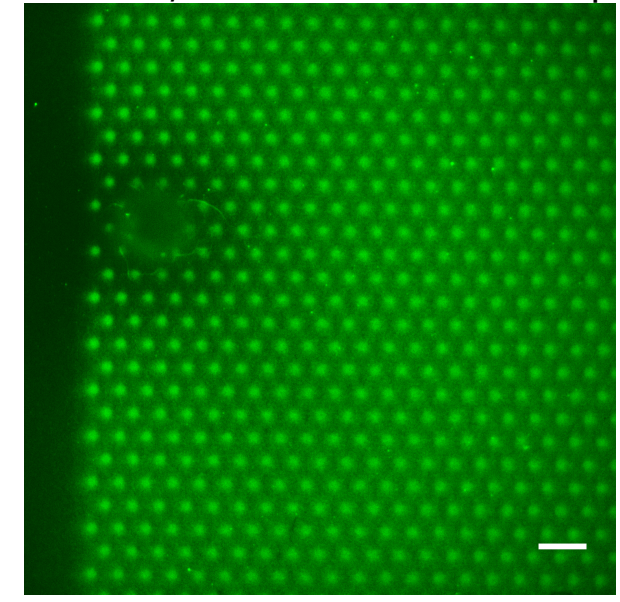

B

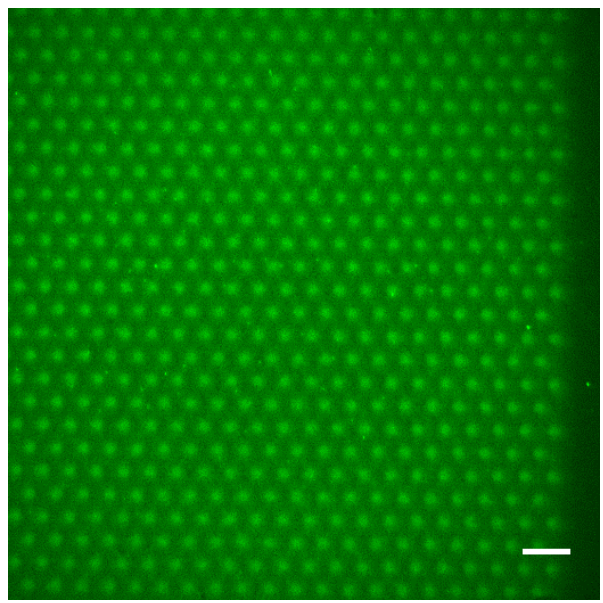

Figure 5.12: POPC:POPS:BP 49.5:49.5:1 membrane patches on PDMS. A: before $R_{6}$ addition; $\mathbf{B}$ : with $R_{6}$ $10 \mu \mathrm{M}$. Scalebars $10 \mu \mathrm{m}$. 
Figure 5.12 shows what this approach brought on a $50 \%$ POPS membrane (also see Section 5.4 .2 p. 112 ff. ). After R6 addition (B), no clear change had occurred, except the previously also observed increased adhesion of vesicles. This suggests that a continuous membrane is crucial for the formation of bilayer stacks induced by R-peptides, and that lipid material from solution is not enough to form stacks.

\subsection{Molecular aspects of stack formation}

In this subchapter, the focus will be on what lipid mixtures comprising the membrane, as well as R-peptides, allow lipid bilayer stack formation. It will commence with observations made with different fluorescent dyes in the lipid bilayer. Then, the focus will be shifted to what peptides and lipid mixtures are able to form stacks, to conclude the experimental results with a determination of dissociation constants of R-peptides.

\subsubsection{Influence of the membrane dye}

From the results presented in Figure 5.10 (p. 103) and Figure 5.11 (p. 104), the suspicion arose that the dye used to visualise the membrane influences the stack formation process. Therefore, $\mathrm{R}_{6}$ was added to POPC:POPG 1:1 membranes with $1 \%$ of different dyes, and the effects were monitored by confocal laser scanning microscopy and fluorescence recovery after photobleaching.

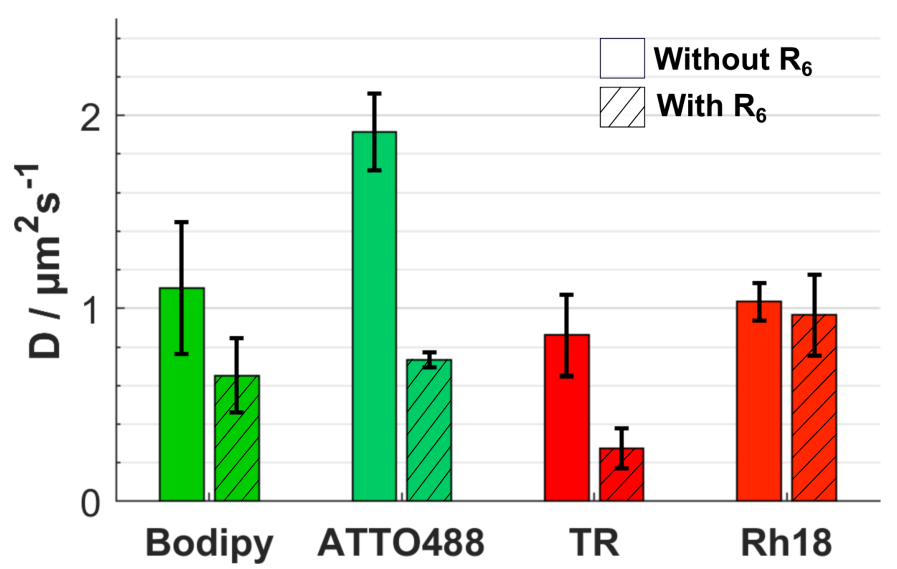

Figure 5.13: Diffusion coefficients for four different membrane dyes. Colored bars represent the mean for at least 3 measurements, error bars present the standard deviation. $R_{6}$ was added to a concentration was $1 \mu \mathrm{M}$, except for ATTO488: there it amounted to $10 \mu \mathrm{M}$. 
A comparison of the diffusion coefficients of the different dyes is presented in Figure 5.13. Four dyes were examined with and without $R_{6}$ (with $R_{6}$, diffusion coefficients were obtained at least 20 minutes after $R_{6}$ addition): The previously used green lipid tail dye Bodipy 500/510 C12-HPC (otherwise called BP or Bodipy), a green headgroup dye ATTO488-DOPE, a red headgroup dye Texas Red (TR), and a membrane staining dye Rh18 (see Materials \& Methods p. 52 for more information). Most of the examined dyes showed a reduction in their diffusion coefficient, but only in case of headgroup dyes ATTO488 and Texas Red, this was significant. $R_{6}$ concentration was $1 \mu \mathrm{M}$, except in case of ATTO488-DOPE (10 $\left.\mu \mathrm{M}\right)$. In the next subsections, observations for the individual dyes are presented.

\section{Texas Red}

Observations made on the membrane containing Texas Red-DPHE are presented in Figure 5.14 ( $p .108$ ). The most striking observation for this dye, was that no stacks were observed, reproducibly (four independent experiments). This is surprising, because Gräb et al. did observe reliable stack formation on Texas Redcontaining membranes[170]. Vesicle adhesion did occur, as was already shown in Figure 5.10 E for added Bodipy-containing vesicles, and was also visible in the experiment presented in Figure 5.14 A (data not shown, after last image at $37: 00)$.

A second observation is that, just like with Bodipy, inhomogeneity of the membrane occurs, which seems to even out over time (Figure 5.14 A). However, the Texas Red dye seems to become more clearly concentrated in grain-like domains, and the rehomogenisation occurs much more slowly (after 40 minutes still incomplete, while typical Bodipy-dyed membranes were homogeneous again after less than 10 minutes).

This slower rehomogenisation is reflected in the behaviour of the Texas Red diffusion coefficient, as already shown in Figure 5.13: if the diffusion coefficient was measured for several times in close sequence after $R_{6}$ addition (shown in Figure 5.14 B), it seemed to decline over time. Despite the low accuracy of such measurements, this consistent trend of decline indicates progressive immobilisation of Texas Red.

All in all, while no stacks were observed, the behaviour of PG-containing membranes with Texas Red was still clearly influenced by $R_{6}$. 

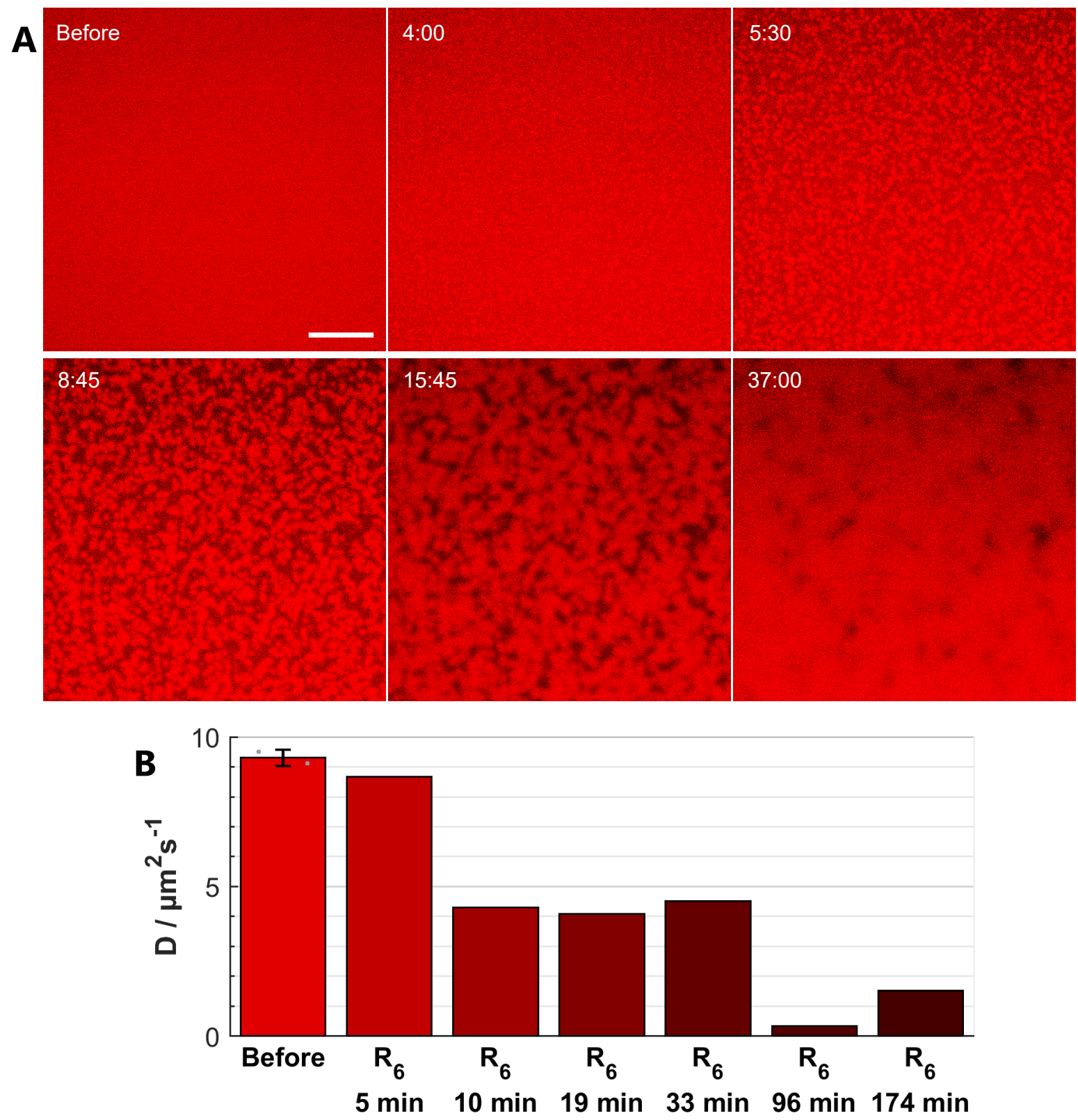

Figure 5.14: Exemplary observations made on a Texas Red and POPG-containing membrane upon $R_{6}$ addition $(1 \mu \mathrm{M})$. A: Time-dependent fluorescence micrograph series where $\mathrm{R}_{6}$ was added between the first two frames. Time indicated in mm:ss signifies the time after $\mathrm{R}_{6}$ addition. Scalebar $20 \mu \mathrm{m}$. B: Series of Texas Red diffusion coefficient deduced from FRAP experiments executed consecutively after $R_{6}$ addition, compared to the diffusion coefficient before $R_{6}$ addition. Below each bar, the approximate start time after $R_{6}$ addition of each FRAP experiment is indicated. 


\section{ATT0488}

The second dye examined was ATTO488-DOPE, a positively charged headgroup dye. Also for this dye, dehomogenisation that evened out over time occurred (Figure 5.15 A), and darker and brighter regions remained, just like for Texas Red in most cases. Both the darker and the brighter regions showed full recovery after photobleaching (Figure 5.15 D and E, respectively), and diffusion coefficients obtained from them were similar (Figure 5.15 F).

Remarkably, darker domains on the membrane seem to form and become more defined over time, with shapes similar to that typically observed for stacks on Bodipy-containing membranes. The darkness of these domains is not consistent: in Figure 5.15 A, they seem to stem from large vesicle-like structures that spread on the membrane, and are brighter at first before turning dark. Also, when bleaching a dark domain (Figure 5.15 B), it seems to be brighter than the surrounding membrane during the first part of the recovery. The fact that these domains show full recovery simultaneously with growth, indicates that they are not membrane defects but rather show behaviour similar to previously observed stacks after bleaching. 

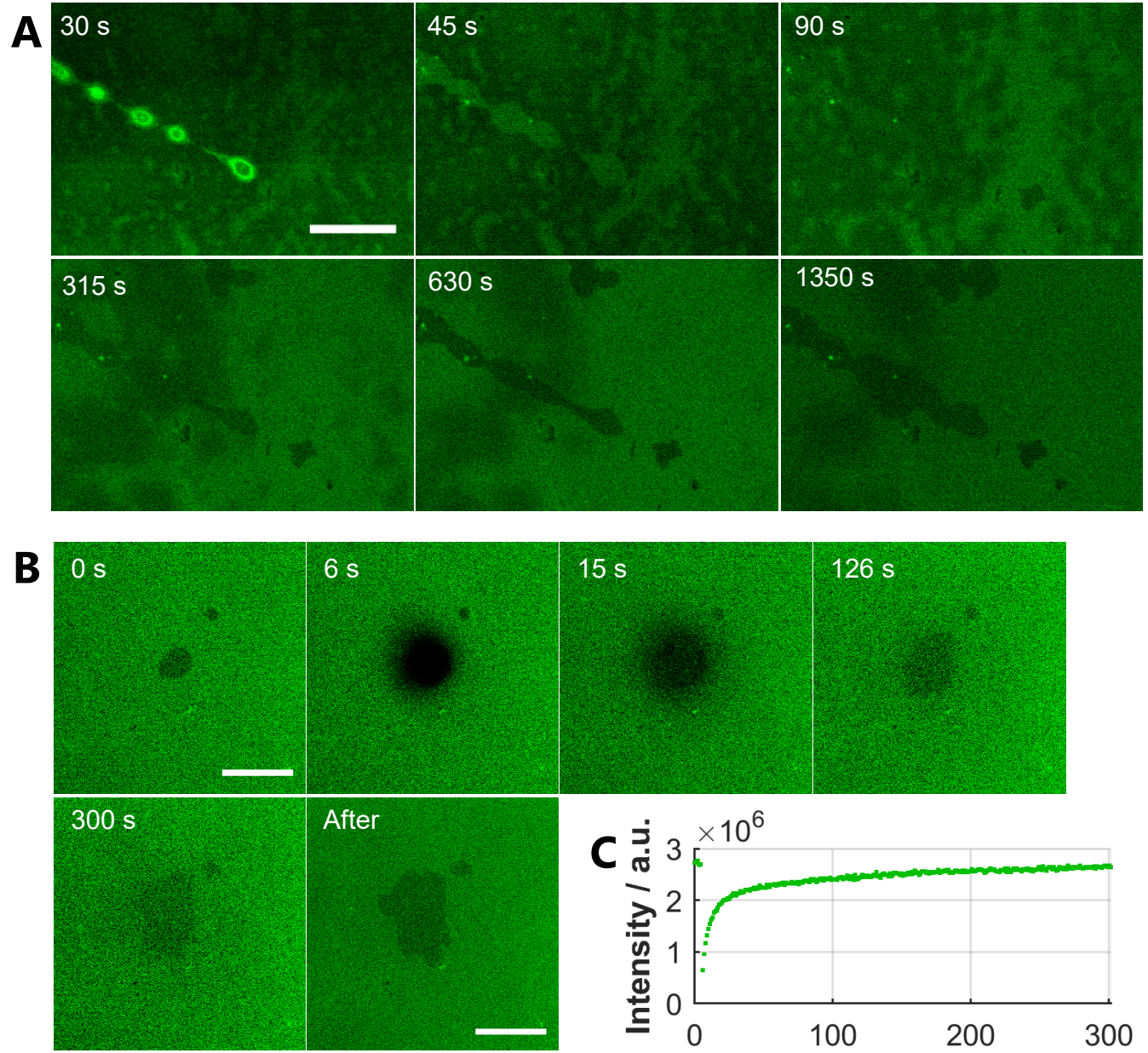

$126 \mathrm{~s}$

D
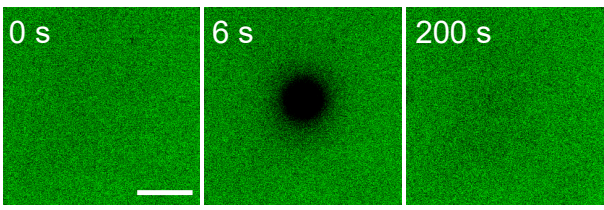

E

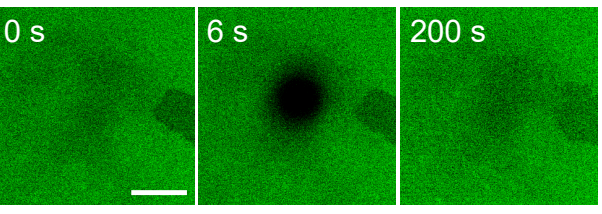

$15 \mathrm{~s}$
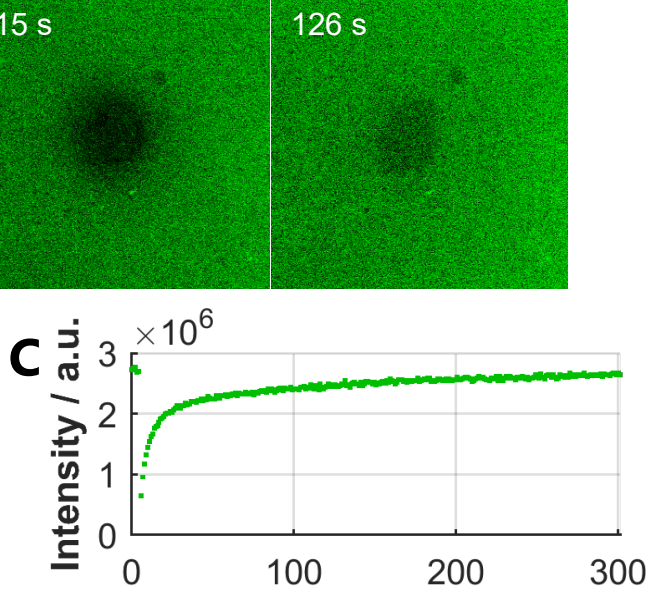

Time / s

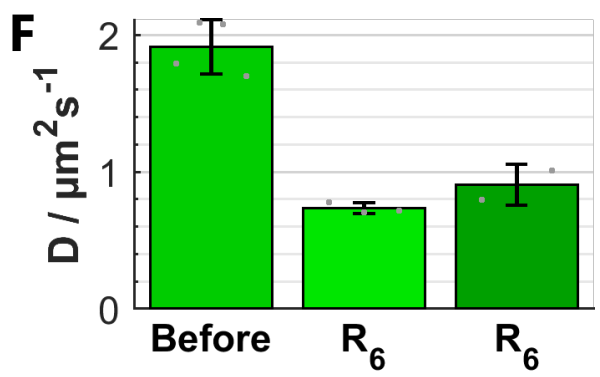

bright dark

Figure 5.15: Results for a POPC:POPG 1:1 bilayer with 1\% ATTO488 upon addition of $10 \mu \mathrm{M} \mathrm{R}_{6}$. A: Fluorescence micrograph series with time indicated in seconds after $R_{6}$ addition. Scale bar $20 \mu \mathrm{m}$. B: FRAP experiment on a darker domain showing full recovery. Time indicated as series recording time in seconds. The last image was recorded after the bleaching series with a higher resolution. Scale bars 10 $\mu \mathrm{m}$. C: Fluorescence intensity over time of the bleached spot in B; D: FRAP experiment on a brighter region of the bilayer, with full recovery. Scale bar $10 \mu \mathrm{m}$; E: FRAP experiment on a darker region of the bilayer, with full recovery. Scale bar $10 \mu \mathrm{m}$; F: Diffusion constants for ATTO488 with $\mathrm{R}_{6}$ of both the darker and brighter regions, as compared to the diffusion constant before $\mathrm{R}_{6}$ addition. 


\section{In-situ staining of membrane stacks with Rh 18}

From results presented previously in this subsection (5.4.1), a concern arose that observed stack formation might largely be caused by the dye used to visualise the membranes. To address this, an experiment was performed in which the membrane was first incubated with $R_{6}$, and then coloured with a red fluorescent membrane staining agent, Rh18. For this dye, stacks were observed again (Figure 5.16), both when $\mathrm{R}_{6}$ was added after staining (data not shown), and when $\mathrm{R}_{6}$ was added first and then Rh18.

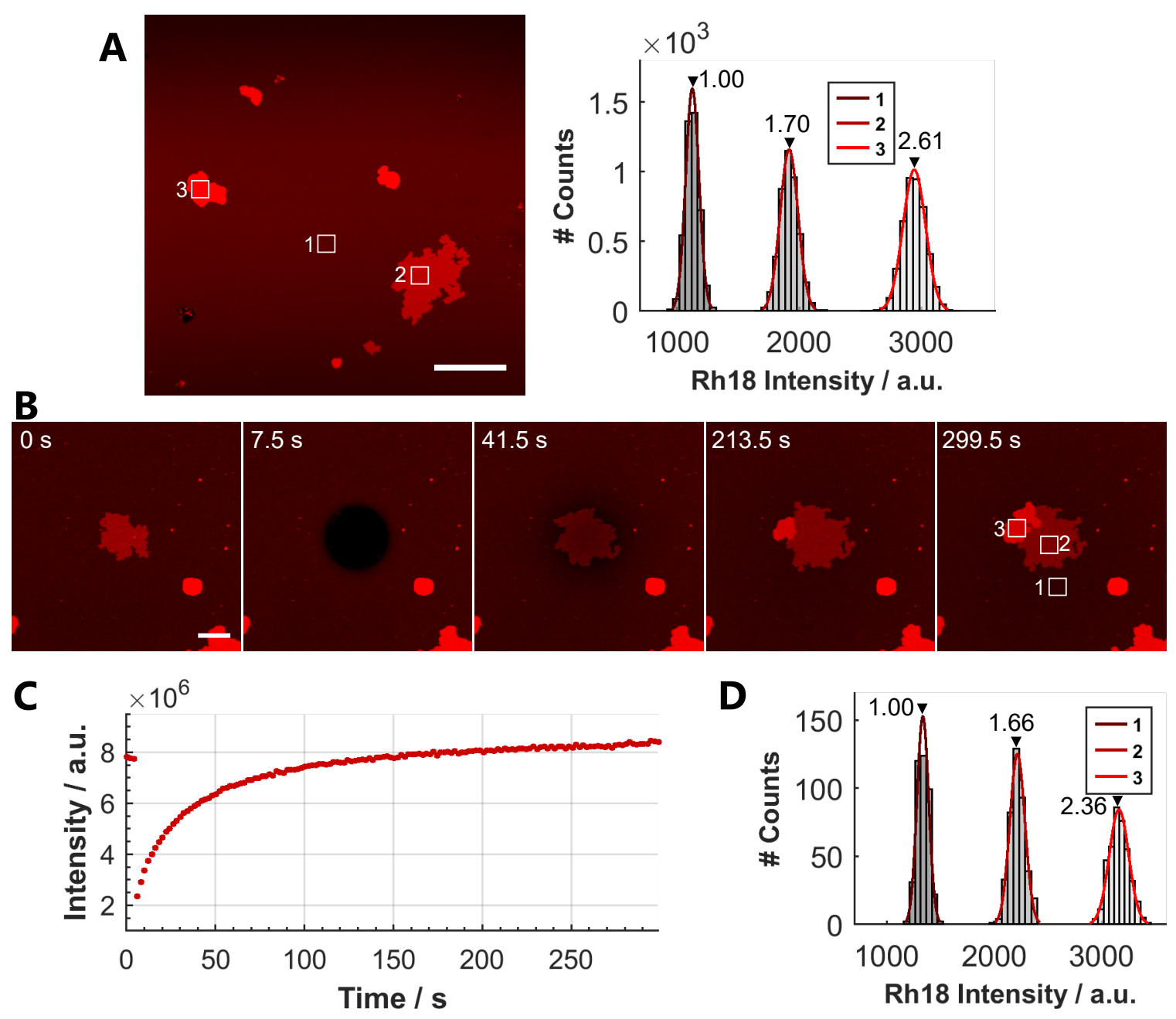

Figure 5.16: Results for in situ POPC:POPG $1: 1$ membrane staining with Rh18 while $\mathrm{R}_{6}$ was already present $(1 \mu \mathrm{M})$. A: Stacks of different intensity levels (left) with a histogram analysis of the indicated square regions (right). Relative intensities are indicated over each Gaussian fit. B: FRAP experiment in which a stack was bleached, and another stack grew on it during recovery; C: Integrated intensity over time of the bleached spot in $\mathbf{B} ; \mathbf{D}$ : Histogram intensity analysis of square regions indicated in the last image in B. Relative intensities are indicated over each Gaussian fit. Scalebar A: $20 \mu$ m; Scalebar B: $10 \mu \mathrm{m}$. Also with this dye, stacks showed recovery after photobleaching, with an example in Figure 5.16 B-E. Despite the slow frame rate and fast scanning speed, significant alteration of the stack occurred during the experiment: a second stack grew on top of it. Therefore, quantitative analysis of this recovery for different 
levels of stacks was not feasible. From the FRAP curve in Figure 5.16 C, recovery seems complete; the continuing increase of fluorescence intensity in the bleached spot was due to the growth of the second stack.

\subsubsection{Peptides and lipids necessary for stack formation}

The second aspect of interest for molecular conditions for stack formation, was the peptides and lipids necessary to cause stack formation on solid-supported bilayers. The focus was especially on the length of R-peptide, bearing in mind that literature showed lamellarisation with $R_{9}$, while this work has shown it for the shorter $R_{6}$ as well. Given that $R_{5}$ was the shortest R-peptide used in literature as a cellpenetrating peptide [35], and $\mathrm{R}_{4}$ has been used as a typical non-cell-penetrating R-peptide in other work for comparison purposes, $R_{4}$ was scrutinised first. Secondly, a lysine hexapeptide $\mathrm{K}_{6}$ was examined, as it has been shown before that lysine peptides are typically less active than their arginine counterparts due to their singly charged sidechain without bridging ability $[8,18,33,37,38]$, although poly-L-lysine has been used for the stepwise formation of membrane stacks [173, 174].

Figure 5.17 shows fluorescence micrographs of stacks discovered on POPC:POPG:BP 49.5:49.5:1 membranes combined with different peptides, along with histograms of intensity levels of indicated square regions. Comparison of these intensity levels to those previously observed with $R_{6}$, together with domain shape, were taken as sufficient indication that stack formation had taken place.

Remarkably, it was discovered that even very short peptides $R_{4}$ and $R_{2}$ can cause stack formation, if in sufficiently high concentration. For $R_{4}$, stable stacks were observed at $100 \mu \mathrm{M}$ peptide concentration (Figure 5.17 B), a factor 100 more than necessary for $R_{6}$. In case of $R_{2}$, another factor 100 higher concentration was necessary: stacks were observed at an $R_{2}$ concentration of $100 \mathrm{mM}$ (Figure 5.17 A). Moving to the lysine peptide, $\mathrm{K}_{6}$ was also able to form membrane stacks, but at the same concentration as $R_{4}, 100 \mu \mathrm{M}$. This is in line with previous observations on lesser binding ability and activity when arginine amino acids are 
A
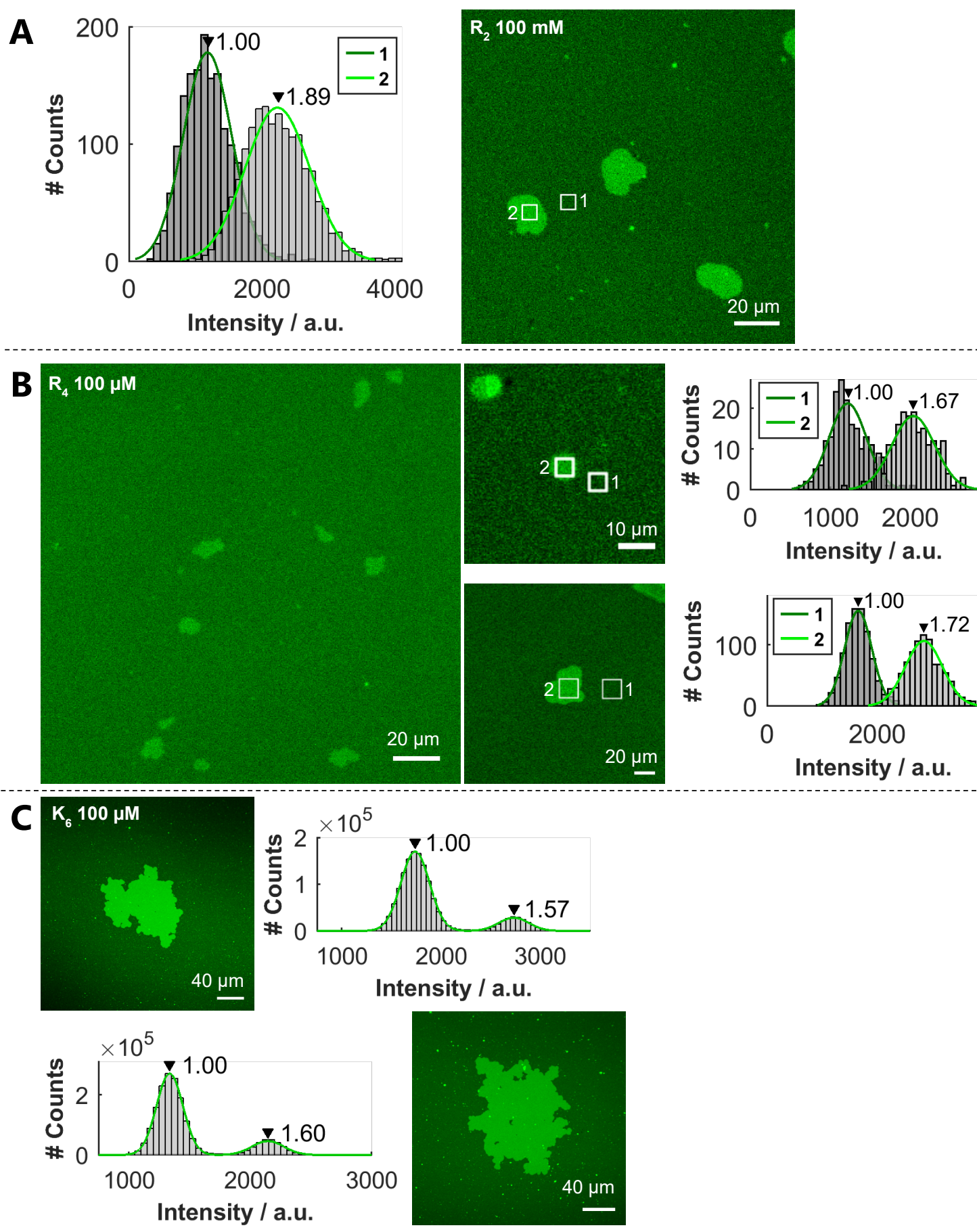

Figure 5.17: Fluorescence micrographs of stacks observed on POPC:POPG:BP 49.5:49.5:1 membranes for different peptides. Scalebar length and peptide indicated in each image. Each fluorescence micrograph is accompanied a histogram plot of pixel intensities of indicated square regions, with a gaussian distribution fitted to each histogram and the relative intensity values for the bilayer and the different stack levels indicated above the histograms. A: $R_{2} 100$ mM; B: $R_{4} 100 \mu \mathrm{M}$; two histograms are given for the two middle images; $\mathbf{C}: \mathrm{K}_{6} 100 \mu \mathrm{M}$. In this case, a histogram of pixel intensities for the whole image next to it is shown, with two gaussian distributions fitted to them.

replaced by lysine $[8,18,33,37,38]$. All peptide combinations showed no significant change of the diffusion coefficient of the Bodipy dye (data not shown). 
Furthermore, similar temporary fluorescence dehomogenisation to that recorded for $\mathrm{R}_{6}$ was observed upon peptide addition (data not shown).

Next, it was examined whether a different negatively charged lipid, POPS, would show the same stack formation behaviour as POPG. Results for a POPC:POPS:BP 49.5:49.5:1 membrane are shown in Figure 5.18. $R_{6}$ showed
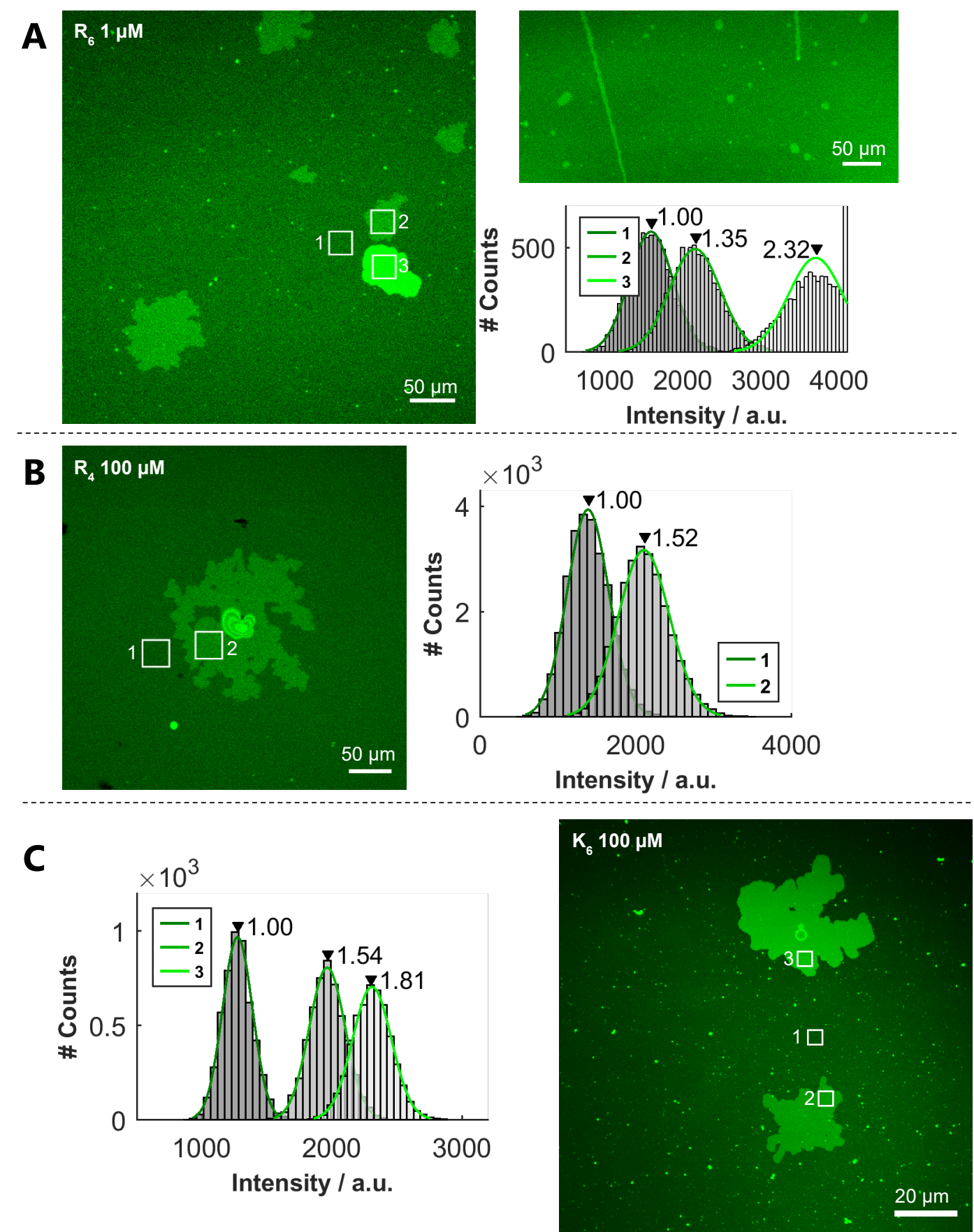

Figure 5.18: Stack formation on POPC:POPS:BP 49.5:49.5:1 membranes by different peptides. A: $R_{6} 1 \mu \mathrm{M}$, bottom right: histogram plot for square regions indicated in the left image; Top right: extensive lineshaped stacks. B: $\mathrm{R}_{4} 100 \mu \mathrm{M}$, histogram of pixel intensities of the indicated square regions on the right; C. K6 $100 \mu \mathrm{M}$, left: histogram of pixel intensities of indicated square regions on the right image, right: fluorescence micrograph of stacks caused by $\mathrm{K}_{6}$ addition. 
reproducible stack formation at the same low concentration of $1 \mu \mathrm{M}$. A marked feature in these membranes was the abundant occurrence of line-shaped stacks (Figure 5.18 A, top right), presumably resulting from tubes directly spreading onto the surface: without R-peptide, these membranes displayed extensive tube formation upon microscopic observation and illumination (data not shown). Otherwise, $\mathrm{R}_{4}$ and $\mathrm{K}_{6}$ also induced stack formation in POPS-containing membranes at the same elevated concentration of $100 \mu \mathrm{M}$ as in POPG-containing membranes. Also analogous to POPG-containing membranes, the diffusion coefficient for Bodipy in POPS-containing membranes did not change significantly in any combination, and stacks showed fluorescence recovery upon photo-bleaching (data not shown).

\subsection{Dissociation constants}

To determine whether the qualitatively determined 'threshold' concentrations for stack formation were connected to the dissociation constant of R-peptides to POPG- and POPS-containing membranes, reflectometric interference spectroscopy (RIfS) was performed. An exemplary RIfS experiment is shown in Figure 5.19, for $\mathrm{R}_{4}$ on a POPC:POPG 1:1 membrane. First, a membrane was formed and rinsed with measuring buffer, to ensure removal of all vesicles. The concentration of Rpeptide was then increased stepwise, until no significant increase in optical thickness was observed anymore (see also Section 3.6, p. 55 ff.). The optical thickness change was fitted for each concentration, to obtain datapoints of concentration versus optical thickness change for each experiment (3.6.1 Data analysis, p. 56).

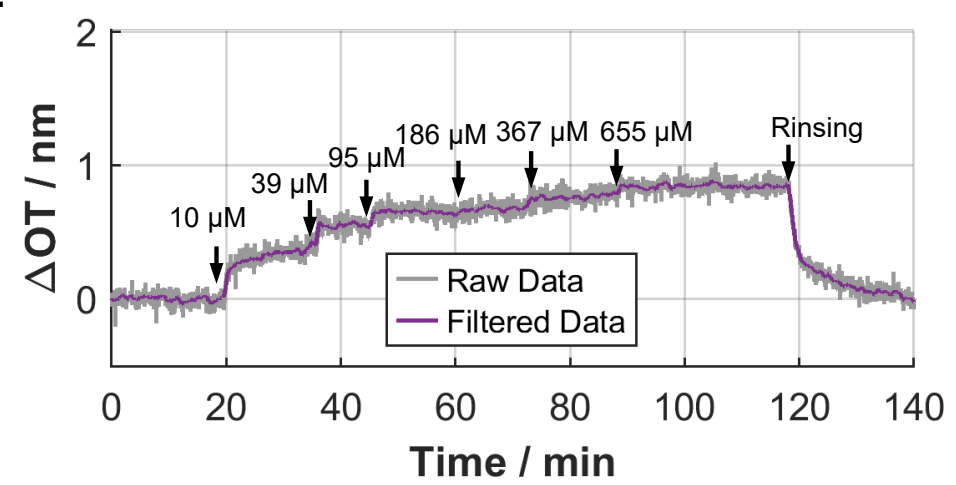

Figure 5.19: Exemplary RIfS experiment to obtain a dissociation constant for $R_{4}$ on a POPC:POPG 1:1 membrane. $\triangle O T$ was plotted against time in minutes. Each arrow indicates a concentration for the peptide for the time after the arrow and before the next arrow. After the maximum concentration, rinsing with measuring buffer was performed, after which the optical thickness returned to normal. 
The optical thickness differences upon R-peptide saturation were measured to be up to 0.9-1.7 $\mathrm{nm}$ (see also Figure 5.20). This value can be converted into physical thickness $d$ by $d=\Delta O T / n$, where $n$ is the refractive index of the peptide layer [128, 130]. This refractive index for the employed R-peptides is unknown to our knowledge, but Zhao et al. demonstrated that for amino acids as well as for two short peptides, the refractive index of a solution varied with ca. $0.2 \mathrm{~mL} / \mathrm{g}$ [175]. If we assume a local concentration of peptide in the peptide layer of $1 \mathrm{M} \mathrm{(5000x}$ maximum global solution concentration), for $R_{6}$ this would be equivalent to a local concentration of ca. $1 \mathrm{~g} / \mathrm{mL}$, given its molecular mass of approximately $1000 \mathrm{~g} / \mathrm{mol}$. Given that the buffer should not have a refractive index much different from that of water $(n=1.33)$, we could assume a refractive index of the peptide layer to be approximately 1.5 . This would make for a physical thickness of $1.7 \mathrm{~nm} / 1.5=1.13 \mathrm{~nm}$.

After obtaining at least two sets of concentration vs optical thickness datapoints for each experiment, datapoints of optical thickness against R-peptide concentration were fitted to an adapted Langmuir model to obtain dissociation constant $K_{\mathrm{D}}$ for each investigated peptide and lipid mixture combination:

$$
\Delta O T=\frac{\Delta O T_{\max } \cdot[\mathrm{R}]}{K_{\mathrm{D}}+[\mathrm{R}]}
$$

Herein, $[R]$ is the R-peptide concentration in $\mu \mathrm{M}, \triangle O T$ is the optical thickness change in $\mathrm{nm}$ relative to the membrane without peptide and $\triangle O T_{\max }$ is the maximum optical thickness change. This $\triangle O T_{\max }$ was also fitted by the Langmuir model, and should be close to the maximum optical thickness change achieved during the experiments.

Obtained dissociation constants for investigated lipid and peptide combinations are given in Table 5.1 ( $p .117$ ). Plots of $\triangle O T$ against R-peptide concentration with a fit to the Langmuir model are shown in Figure 5.20 (p. 118) and Figure 5.21 (p. 119) for POPC:POPG $1: 1$ and POPC:POPS $1: 1$, respectively. In general, the Bodipy dye does not seem to influence the dissociation constant significantly. Furthermore, dissociation constants in POPG and POPS-containing membranes are approximately equal for $R_{6}$. In case of $R_{6}$, fitting data to a Hill-Waud model (Equation (5.2), [145, 146]) delivered significant results for both dissociation constants and Hill Coefficient $m$. The Hill coefficient is descriptive of binding cooperativity. The values obtained are mostly smaller than 1 , which indicates 
negative cooperative binding. This is on par with results obtained by Robison et al. for $\mathrm{R}_{9}[36]$.

$$
\Delta O T=\frac{\Delta O T_{\max } \cdot[\mathrm{R}]^{m}}{K_{D}{ }^{m}+[\mathrm{R}]^{m}}
$$

The trend that was observed for stack formation, is also observed here in the dissociation constants: dissociation constants increased for shorter R-peptides. However, the dissociation constants for $\mathrm{R}_{6}$ and $\mathrm{R}_{4}$ did not differ as much as stack formation behaviour differences ( $1 \mu \mathrm{M}$ vs $100 \mu \mathrm{M}$ ) would suggest. Furthermore, the dissociation constant for $\mathrm{R}_{2}$ was around $10 \mathrm{mM}$, and at this concentration no stack formation could reproducibly be observed.

Table 5.1: Dissociation constants obtained for investigated lipid mixtures and R-peptides, with and without $1 \%$ Bodipy dye. In case of $R_{6}$, RIfS data could additionally be fitted to a Hill-Waud model $[144,145]$, to obtain the cooperative unit $m$ for binding.

\begin{tabular}{|c|c|c|c|c|}
\hline Fit to Langmuir model & \multicolumn{2}{|c|}{$\begin{array}{l}\text { With dye } 1 \% \\
K_{\mathrm{D}} / \mu \mathrm{M}\end{array}$} & \multicolumn{2}{|c|}{$\begin{array}{l}\text { Without dye } \\
K_{\mathrm{D}} / \mu \mathrm{M}\end{array}$} \\
\hline POPC:POPG 1:1 + $\mathrm{R}_{2}$ & \multirow{3}{*}{\multicolumn{2}{|c|}{$\begin{array}{c}(21.7 \pm 6.0) \times 10^{3} \\
48 \pm 13 \\
7.6 \pm 1.5\end{array}$}} & \multirow{3}{*}{\multicolumn{2}{|c|}{$\begin{array}{c}(12.9 \pm 2.8) \times 10^{3} \\
18.6 \pm 3.7 \\
8.0 \pm 1.7\end{array}$}} \\
\hline$+R_{4}$ & & & & \\
\hline$+R_{6}$ & & & & \\
\hline POPC:POPS 1:1 + $\mathrm{R}_{6}$ & \multicolumn{2}{|c|}{$7.08 \pm 0.84$} & \multicolumn{2}{|c|}{$26.8 \pm 3.0$} \\
\hline Fit to Hill-Waud model & $K_{\mathrm{D}} / \mu \mathrm{M}$ & $n$ & $K_{\mathrm{D}} / \mu \mathrm{M}$ & $n$ \\
\hline POPC:POPS 1:1 + $\mathrm{R}_{6}$ & $8.0 \pm 1.3$ & $0.863 \pm 0.091$ & $24.0 \pm 3.5$ & $1.10 \pm 0.12$ \\
\hline POPC:POPG 1:1 + $\mathrm{R}_{6}$ & $10.6 \pm 4.4$ & $0.74 \pm 0.15$ & $11.8 \pm 5.5$ & $0.70 \pm 0.16$ \\
\hline
\end{tabular}



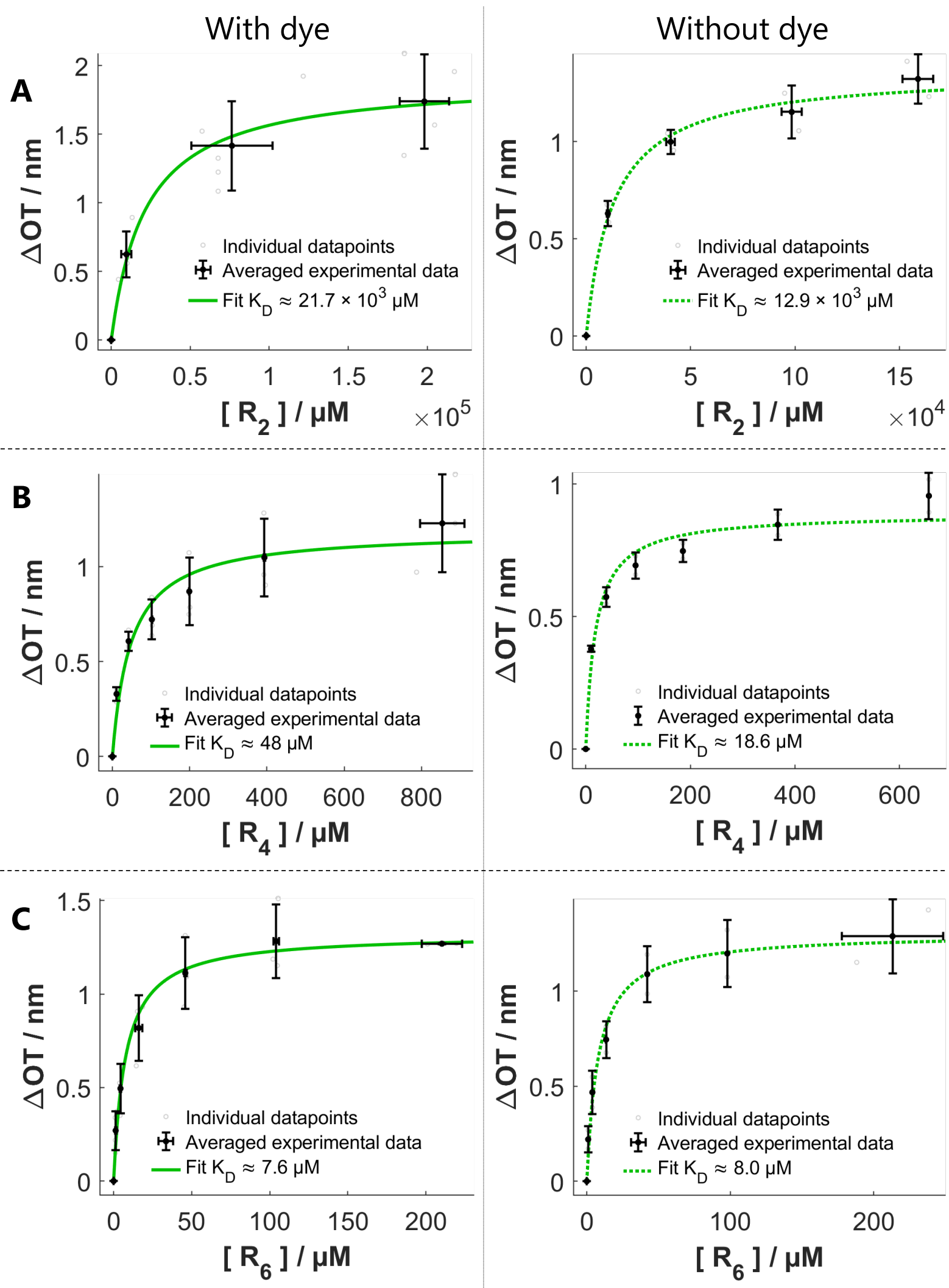

Figure 5.20: Plots of optical thickness change $\triangle O T$ against R-peptide concentration in $\mu \mathrm{M}$ for POPC:POPG 1:1 membranes, with (left plots) and without (right plots) 1\% Bodipy dye. Data of at least two different experiments (experimental datapoints light grey circles) were averaged and indicated with black error bars. If measurement concentrations were close together, they were averaged too, and hence some horizontal error bars are shown. Dissociation constants obtained by fitting to a Langmuir model are indicated in the legend of each plot. $\mathbf{A}: R_{2} ; \mathbf{B}: R_{4} ; \mathbf{C}: R_{6}$. 

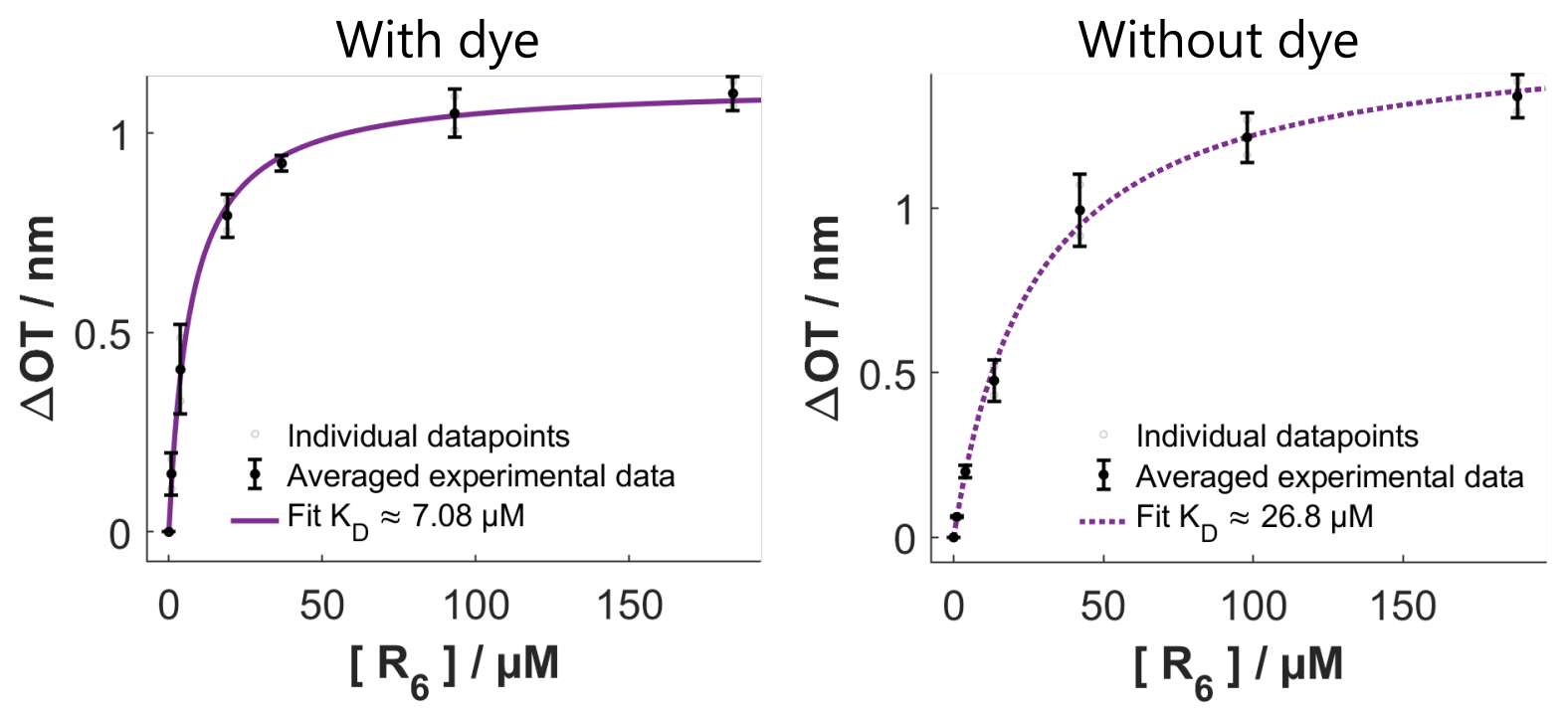

Figure 5.21: Plots of optical thickness change $\triangle O T$ against $R_{6}$ concentration in $\mu M$ for POPC:POPS 1:1 membranes, with (left plot) and without (right plot) 1\% Bodipy dye. Data of at least two different experiments were averaged and indicated with error bars. Dissociation constants obtained by fitting to a Langmuir model are indicated in the legend of each plot. 


\subsection{Discussion R-peptide induced stack formation}

After having presented all results on characterisation of membrane domains, the implications of these results will be discussed here. The results show remarkable similarities but also marked differences with earlier work, especially the work of Gräb et al., which will be indicated as we move to the different aspects of this discussion. It will commence with the structural characterisation of the membrane stacks, followed by their mechanism of formation, and lastly, molecular requirements for their formation. Especially in the last part, further similar phenomena mentioned in literature will be discussed. See Chapter 6 ( $p .137$ ff.) for an overarching discussion of all results from Chapter 4 and 5 in mechanistical and biological context.

\subsubsection{Structure of observed domains}

When comparing three possible models for the observed domains (Figure 5.22) analogously to Gräb's work [170, 172], the presented data fit best the hypothesis that observed domains are lipid bilayer stacks, with mostly the same arguments as Gräb et al. presented: it fits the presence of multiple discrete fluorescence levels (Figure 4.15 p. 85), the multi-nm height difference between main membrane and domains (Figure $\mathbf{5 . 1}$ p. 92), as well as the double breakthroughs that were observed on a domain (Figure $\mathbf{5 . 2}$ p. 93). Additionally, the present work has very clearly correlated the discrete fluorescence levels with discrete height levels by means of correlating fluorescence and AFM imaging: the first level presents a factor 1.33 in fluorescence intensity (factor $1.3-1.8$ overall) and $8 \mathrm{~nm}$ height difference; the second level presents at least a factor 1.6 increase in fluorescence

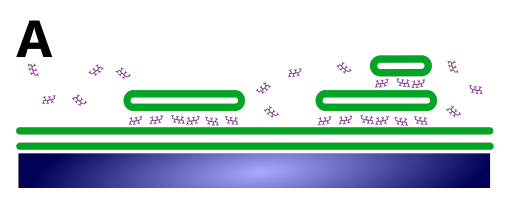

B
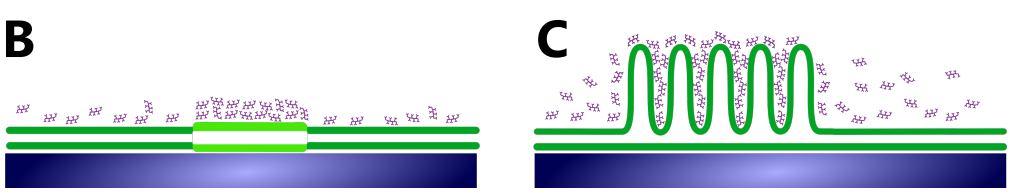

Figure 5.22: Three models for more highly fluorescent domains on POPG- or POPS-containing bilayers in the presence of R-peptides. A: The domains are bilayer stacks; $\mathbf{B}$ : The domains are lipid domains which the fluorophore prefers over the main bilayer, with R-peptide accumulated on top; $\mathbf{C}$ : The domains consist of folds or tubes of regular length, clustered by R-peptides. 
intensity compared to the main membrane and ca. $16 \mathrm{~nm}$ in height difference (Figure 5.1 p. 92).

One could argue that the observed height difference between the main bilayer and the first level of stacks, should represent two bilayers, since it is a rather large difference for just one bilayer (typically 3.5-5 nm, [105, 154, 169-171]). However, several other data in this work show that one bilayer per stack is not an invalid assumption. The estimated full membrane thickness as obtained from breakthrough force curves in Figure 4.2 C (p. 66) of the previous chapter $(p .66)$, does show values of at least $6 \mathrm{~nm}$, and rather higher values for POPG-containing bilayers. Since the images showing an $8 \mathrm{~nm}$ height difference were acquired by essentially recording short-range indentation curves (see Methods Background p. 37), it is safe to assume that this $6 \mathrm{~nm}$ height difference is at least a large portion of that detected in the images. The additional $2 \mathrm{~nm}$ could be assumed to stem from a layer of R-peptides, especially if we reasonably assume a double layer of peptides connects the two lamellae in a stack: ca. $1 \mathrm{~nm}$ per fully saturated peptide layer was estimated from the RIfS data in this work.

Two other possible models for the structure of the observed domains are presented in Figure 5.22 B \& C (p. 120), but they can be rejected analogously to Oliver Gräb's work: The model assuming phase-separation-like behaviour with a layer of peptides on top (Figure 5.22 B p. 120) could not account for the observed height differences, given that phase separation does not account for more than $1.5 \mathrm{~nm}$ $[156,157,168,169]$ and the peptide layer not much more than $1 \mathrm{~nm}$ (according to presented data in Chapter 5.5 Figure 5.19 p. 115). Apart from this most important discrepancy, this model is difficult to connect with the observation of up to four discrete fluorescence levels. This is also the main argument against the third possible model, which comprises local domains of short tube-like folded structures interconnected by R-peptides (Figure $\mathbf{5 . 2 2}$ C p. 120): there is no conceivable reason why perpendicular tube-like structures would result in the observation of discrete and overlapping height and fluorescence levels.

The next question to address, is the nature of the connection between stacks and the main membrane. Fluorescence recovery after photobleaching experiments on stacks have clearly proven that a connection must exist: if there were no connection, recovery would not reach more than $50 \%$ and the stack would not 
visibly return to the fluorescence image, while presented data showed nearly full recovery (Figure $\mathbf{5 . 3}$ p. 94).

Figure 5.23 A-C shows three possibilities for the nature of the inter-bilayer connections. The first option (Figure 5.23 A) involves stacks stemming from local tearing and subsequent folding of the bilayer. No tearing of the membrane was observed by either fluorescence or AFM images. Furthermore, tearing is not expected to result in a symmetric fluorescence recovery profile after bleaching (Appendix 10 p. XXXII) [91, 150,176], and therefore does not fit the obtained data well.

A

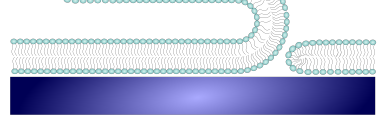

B

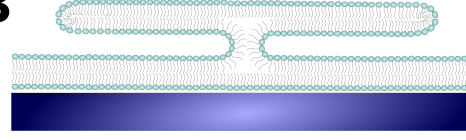

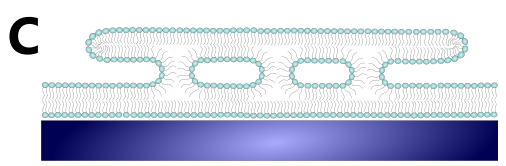

$\mathbf{E}$

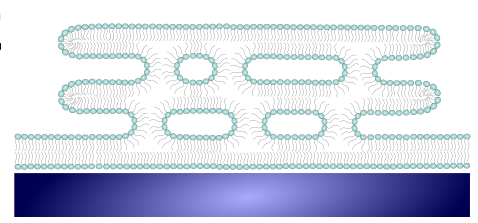

Figure 5.23: Models for the nature of connections between the lipid stacks and the main bilayer, in general $(\mathbf{A}-\mathbf{C})$ and specifically for the homogeneous stacks of the second fluorescence level (D-E). A: the membrane has local tears and folds; B: A single hemifusion stalk-like connection between bilayer and stalk; C: Multiple stalk-like connections for a single stack; $\mathbf{D}$ : The homogeneous stacks of the second fluorescence and height level stem from a local lift-off and folding of both bilayer leaflets; $\mathbf{E}$ : The homogeneous stacks of the second fluorescence and height level consist of exactly aligned single bilayer stacks connected by multiple stalk-like junctions.

A similar argument based on the nature of the recovery profile of a stack was previously brought against the model of a single connection between the main membrane and the stack (Figure 5.23 B) in Gräb's work [170, 172]: if diffusion were restricted by a single stalk-like bottleneck, the fluorescence profile during recovery is not expected to show the uniformity that was observed in Appendix 10 (p. $X X X I I)$, which does not differ much from that of a single bilayer. In a single bilayer, radially symmetrical diffusion is dictated by Fick's laws[177] and models derived by Axelrod et al. [176], and if interbilayer diffusion were restricted, radial diffusion from the location of the bottleneck would be observed.

Furthermore, it can be reasoned that the size of the junction between two bilayers must be much larger than a single stalk-like connection, based on earlier work. Savić et al. [91,150] have shown in simulations correlated with experiments that the mean time constant $T$ deduced from FRAP recovery data is proportional to the 
size of the contact zone between two bilayers, in the context of fusion pairs of membrane-covered silica beads. In case of this work, the contact zone of bleaching of a single bilayer can be taken as the size of the bleached spot, in the order of $10 \mu \mathrm{m}$ in the given examples. The size of the contact zone in case of a single stalklike connection, would be similar to that of a typical membrane thickness, not larger than $10 \mathrm{~nm}$. Therefore, around a factor 1000 difference in mean time constant $T$ should be expected if a single connection is assumed. However, in this work, only roughly a factor two per stack level is observed (Figure 5.4 p. 95). According to this reasoning, many connections must exist between the membrane lamellae, so that essentially twice the amount of material diffuses under the same restrictions. Proof for this hypothesis could in future work be obtained from simulations similar to those performed by Savić et al. [91, 150] on fusion pairs of membrane-covered beads.

One more question to be addressed concerning the nature of the connections between stacks and main membrane, is the explanation for the appearance of seemingly uniform stacks of the second height and fluorescence level. The uniformity of these second level stacks would let one suspect that they stem from a different nature of connections, especially combined with their relative instability compared to first level stacks (Appendix 10 p. XXXIII). These observations could be explained by a model as illustrated in Figure 5.23 D (p. 122), where a portion of the bilayer is folded upon itself, rather than the hemifusion-like model in $\mathbf{C}$ and $\mathbf{E}$.

Despite intuitively suiting the observations better than the illustration in Figure 5.23 E, this model in Figure 5.23 D is unlikely to be valid for three reasons. One is that lifting the entire membrane from its substrate would take a significant amount of energy. This is illustrated by the work of Koçun and Janshoff [87], demonstrating forces involved in pulling tethers from pore-spanning membranes. Therefore, the existence of multiple of such connections, as strongly hypothesised above, would be difficult to reconcile, which is the second reason this model poorly suits the data. Thirdly, several fluorescence observations have shown different overlapping levels sharing one stack border, which indicates that the model in Figure 5.23 E is the most valid after all. 
Taking into account all data and above discussion, we come to the following model to describe the observed membrane domains caused by R-peptides (Figure 5.24): R-peptides connect stacks of membranes, amounting to a height of ca. $6 \mathrm{~nm}$ each, with a ca. $2 \mathrm{~nm}$ layer of R-peptides between them. Multiple connections between stack layers exist, in the form of stalk-like bottlenecks.

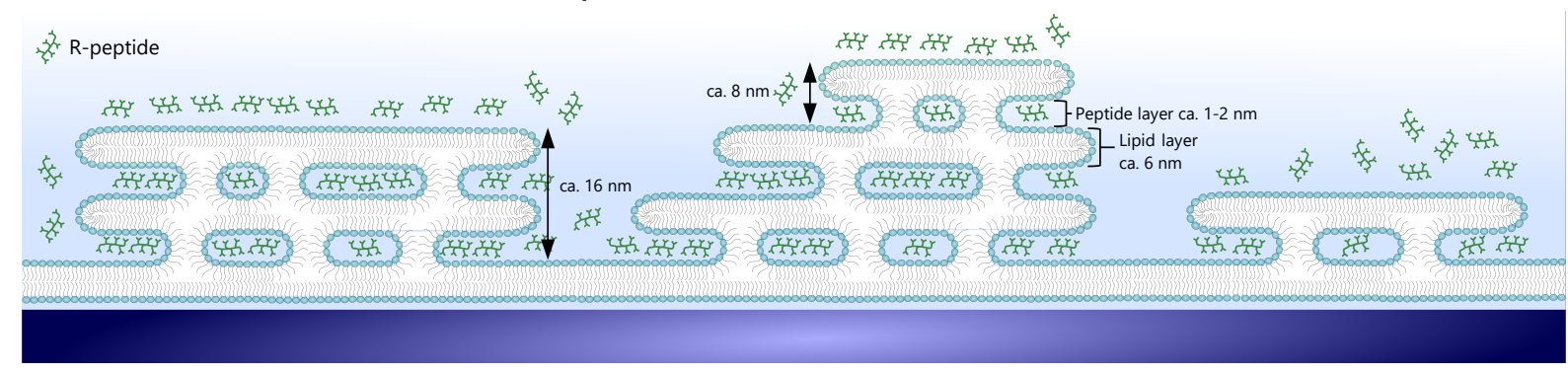

Figure 5.24: Final hypothesis for the structure of R-peptide induced membrane stacks. The height differences indicated are those that were detected by our methods (AFM and RIfS), and comprise $6 \mathrm{~nm}$ for each bilayer and ca. 1-2 nm for the peptide layer between the stacks. This makes for an $8 \mathrm{~nm}$ difference detected per stack level by AFM, and $16 \mathrm{~nm}$ for double stacks. Stacks are connected by multiple stalk-like bottlenecks.

\subsubsection{Stack formation mechanism}

Growth of stacks out of the original bilayer should result in tearing of this bilayer, if no other conditions are changed or no extra lipid material is added: lipid bilayers can only be expanded by $3-5 \%$ of their original size[178], which is unlikely to account for the amount of stacks observed. However, in this work as well as in Gräb's work [170, 172], no tearing of the membrane was observed during stack formation. Therefore, Figure $\mathbf{5 . 2 5}$ presents three possible models for the mechanism of stack formation, with focus especially on the origin of the apparent addition of extra lipid material to the membrane.

The first model that will be discussed, is stack formation by direct spreading and fusion of vesicles from solution onto the main membrane (Figure 5.25 A). Gräb et al. concluded that this was the main mechanism of stack formation induced by long-chain polyamines $[170,172]$. This was mostly concluded from an experiment in which a population of Texas Red-stained vesicles was added to an ATTO488containing membrane: in this dye combination, exclusively Texas Red-containing stacks were observed. Hence it was deduced that stack formation involves direct spreading from vesicles from solution onto the main membrane. 


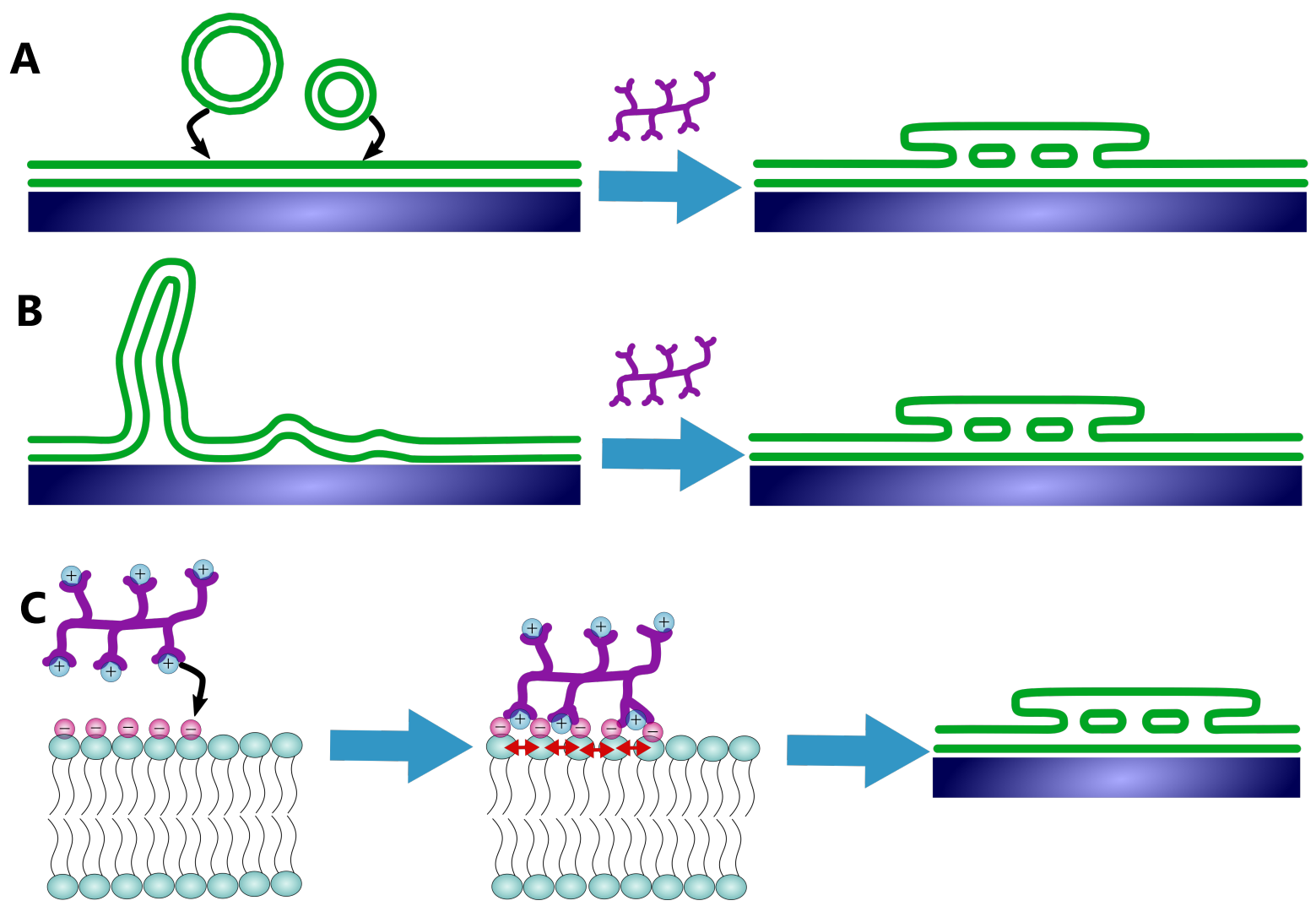

Figure 5.25: Three models for the mechanism of stack formation and the origin of the necessary lipid material. A: Vesicles from solution spread onto the solid-supported bilayer by fusion mediated by Rpeptides; $\mathbf{B}$ : There is excess material present in the bilayer from thermal expansion due to microscopy illumination and R-peptides mediate spreading of this tube-like excess material into flat structures (stacks); C: By binding via charge-charge electrostatic attraction, R-peptides increase the average area per lipid of at least the liquid-facing bilayer leaflet, resulting in excess lipid material. R-peptides then favour arrangement of this area surplus into stacks by electrostatic bridging.

Four observations in this work indicate that fusion of vesicles from solution is at least part of the stack formation mechanism and at least part of the extra lipid material originates from vesicles. When Texas Red-containing vesicles were added to the green membrane before $\mathrm{R}_{6}$-addition, weak TR-fluorescence had appeared throughout stacks and membrane. This indicates that lipid material is indeed added from solution. Also, small disk-shaped stacks on top of larger stacks were observed, which indicate direct spreading from solution. The third observation was disappearance of adhered vesicles in Figure 5.9 A ( $p .100)$, presumably having fused into the main membrane, again showing lipid material entering the main membrane, if not directly at a stack formation site. Lastly, direct observation of a vesicle from solution apparently fusing with an already present stack was shown (Figure 5.8 p. 99).

However, assuming stack formation by direct spreading of vesicles from solution onto the main membrane as the main mechanism of stack formation, does not 
rhyme well with two other experimental observations. Firstly, the anecdotal observation of a vesicle from solution fusing with the membrane over the course of 10 seconds, suggests already that this process, while taking place, seems too slow to account for all observed stack growth. The second problem is the observation that under epifluorescence illumination, fast stack growth as well as new formation takes place. Continuous focused illumination of a sample generates heat input, and thus it can be assumed that the local temperature of the observed section of the membrane rises, albeit not possible to measure. A rise in temperature has been shown to increase the rate of membrane fusion [179-181], as well as membrane spreading [182] in different systems. However, we reason that because directly observed vesicle fusion from solution under continuous illumination appeared very slow, stack formation rate increase with heat input should not be observed if it stems from vesicle spreading alone.

Growth with temperature could however be well connected with the second stack formation mechanism proposal (Figure 5.25 B): the lipid bilayer already contains excess material, an "internal pool" of lipid material as defined by Gräb et al [170, 172]. R-peptides cause the flat spreading of this material onto the surface by bridging an attractive interaction between two bilayers. This is a reasonable model because the lipid mixtures that showed stack formation, those containing POPG or POPS, showed rapid and extensive tube formation upon illumination in the absence of R-peptides (example in Figure 4.11 A, right image, p. 80). This tube formation is a direct consequence of membrane expansion upon temperature increase[105]: the thermal expansion causes an excess of lipid material, which has been shown before to result in tube formation[183]. However, tubes were barely observed in the presence of R-peptides, which makes it reasonable to assume that membrane stacks are the equivalent of tubes when R-peptides are present. This assumption, in turn, fits the observation of stack growth with illumination very well. It also fits the observation of tubes at the stack borders (Figure 5.9 A), as well as the splitting off of vesicle-like structures (Figure 5.9 B)

This stack formation mechanism also suits well the apparent necessity of a continuous membrane for the formation of stacks (Figure 5.12): a noncontinuous membrane per definition does not have excess material, and can therefore not provide the material for stacks. It could only absorb material from vesicles in the above-standing solution, but according to the experimental observations, that was not enough. 
A third model that we discuss here (Figure $5.25 \mathrm{C} p .125$ ), is related to the previously discussed model $\mathbf{B}$, by the involvement of excess lipid material. Here however, R-peptides increase the area per lipid by binding to the PG- or PSheadgroups, thereby increasing overall membrane area of at least the solutionfacing leaflet. This results in an excess membrane area, which then is then spread onto the main membrane in the form of stacks, facilitated by the positively charged peptide layer. This model would especially well suit the observation of stack formation directly after R-peptide addition, made under non-continuous illumination by confocal laser scanning microscopy. These recordings (example Figure $5.6 p$. 97) were made with 1 frame per 10-15 seconds and by precise laser scanning. Hence, overall heat input is assumed to be insufficient to be responsible for stack formation purely described by model B. This model C could also provide an explanation for the observation of temporarily inhomogeneous fluorescence of the main membrane, as was often observed briefly after peptide addition: Rpeptide adsorption would need time to reach equilibrium. During that equilibration time, it is conceivable that the dye distribution becomes temporarily inhomogenous, until a final spacing of lipids is established. This area per lipid increase caused by R-peptides is supported by the simulation data presented in Section 4.1 on $p .62 \mathrm{ff}$. Further experimental work would need to be done to prove the validity of this model more definitely, especially the increase in overall area.

\subsubsection{Molecular requirements for stack formation}

Here, we will discuss the materials that are required to observe stack formation, based on the findings presented in this work. First, the influence of the membrane dye to visualise the membrane will be discussed. Afterwards, we conclude this chapter with a discussion on materials that cause stack formation, in terms of what lipids are necessary, but also the type of molecule that is able to induce stack formation in these lipid mixtures.

\section{Influence of membrane dye}

This work has shown clear stack formation for different R-peptides and lipid compositions (see next subsection), when the membrane was stained with the lipid tail dye Bodipy (see 3.1.3 Dyes p. 45). These results make it reasonable to assume 
that stack formation is mainly driven by attraction between the positively charged guanidine moieties in R-peptides and the negatively charged lipid headgroups (see next subsection). Therefore, it is unlikely that a lipid tail dye with a PC headgroup would be the driving force between stack formation.

However, in RIfS experiments with vesicles still present (Figure 5.11 p. 104), different behaviour was observed with dye than without dye: upon addition of 20 or $30 \mu \mathrm{M} \mathrm{R}{ }_{6}$, dye-containing membranes showed a much stronger optical thickness increase (more than $4 \mathrm{~nm}$ ) over the relatively short time of an hour, while without dye, this was not more than ca. $2 \mathrm{~nm}$. Therefore, eventual concerns that can be raised based on the different behaviour for different dyes or presence vs. absence of dye, as well differences in observations with respect to Gräb's work, will be addressed here.

First it has to be mentioned, that different observable behaviour of a lipid bilayer model system depending on dye used [172, 184-187], or in general, dyes behaving differently in different conditions[188, 189], is not an unknown observation. However, in most model membrane studies, the influence of a few percent of dye in the overall lipid composition is assumed to be negligible, and therefore, emergence of such differences in a specific study can seem alarming. For this reason, it was decided to discuss likely explanations for behaviour of the three other dyes tested in the present work.

We start this part of the discussion with Texas Red-DHPE, which is an overall negatively charged lipid (see 3.1.3 Dyes p. 45). No stacks were observed in this work, only progressive immobilisation of the dye over time. This could perhaps be explained by interaction with the R-peptides' positive charge, and a peptide layer therefore rendering the dye immobile. The validity of this assumption would need to be tested by more suitable methods.

For Texas Red-stained membranes, the observations in this work were however clearly different from those in Gräb's work [170, 172], in which Texas Red was used as the main dye to visualise lipid membrane stacks and no clear immobilisation was observed. These discrepancies could possibly be explained by differences in experimental systems, which are known to influence dye behaviour [184]. First of all, only $0.5 \%$ of dye was used, while this work used $1 \%$ of dye, to maintain consistency in lipid compositions. Additionally, the further lipid composition of Gräb's system was different: beside a small amount of POPC and 
POPG, several plant glycolipids (MGDG, DGDG, SQDG) were part of their composition. Furthermore, a different solid support was used: mica. Different surface properties have been shown to influence bilayer[74, 190] and dye[188, 189] behaviour.

The next dye that was evaluated, was ATTO488-DOPE. Given two sulfonic acid moieties, it seems that it should be negatively charged at neutral $\mathrm{pH}$, but pKa data are not known to our knowledge. However, it was mentioned to be neutral and not even zwitterionic in a study on electrostatic interactions of fluorescent molecules with dielectric interfaces [189]. This dye showed some evidence of immobilisation (significantly lower diffusion coefficient) in this work, as well as seemingly permanent dehomogenisation and formation of discrete darker domains. These darker domains were ruled out to be membrane defects by three observations: first of all, they could be bleached to even lower intensity during a FRAP experiment. Secondly, they showed full recovery after photobleaching, and even growth. Growth of membrane defects upon intense irradiation (bleaching) is unlikely, since input of heat rather causes thermal expansion of the membrane and hence possible closure of defects.

We hypothesise that the membranes with ATTO488-DOPE did form stacks upon $\mathrm{R}_{6}$ addition, but that the dye was partially excluded from the membrane-stack interface and therefore not able to diffuse into stacks either. As illustrated in Figure 5.26, this would be caused by steric hindrance as a consequence of the close distance between the main membrane and the stack, bridged by R-peptides.

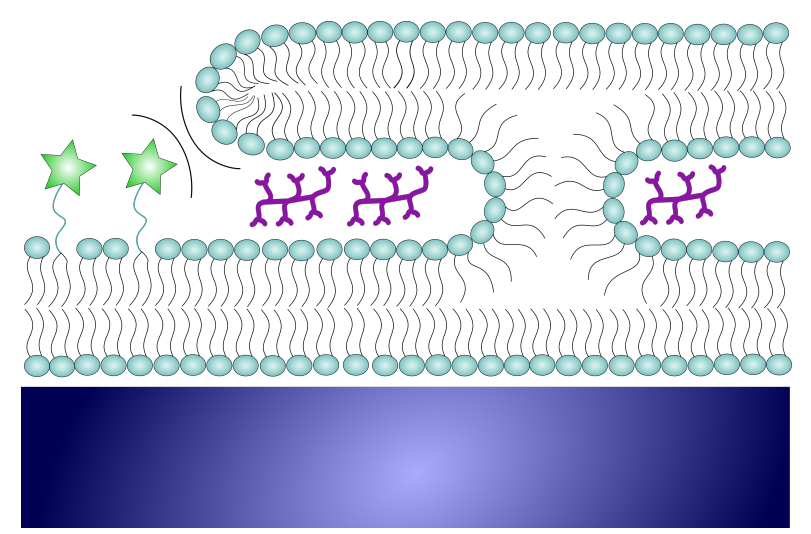

Figure 5.26: Hypothesis for dark domains observed with ATTO488-DOPE as a dye; The dye (green star) is less able to enter the bilayer-stack interface that is bridged by R-peptides because of its long linker region (teal line) and bulky dye structure.

The motivation is that ATTO488 has the longest linker from lipid headgroup to dye molecule of all dyes investigated, due to its linker that is twice as long as for other headgroup dyes (see Figure 3.2 p. 45)). Furthermore, the size exclusion concept 
at bilayer interfaces has been proven by Schmid et al. [191]. They showed that at bilayer-bilayer interfaces between giant vesicles, a longer mCherry-labelled nonbinding protein was excluded from the contact zone, which was bridged by a shorter GFP-labelled protein. More experimental proof is necessary to establish the validity of this concept in case of ATTO488. If interest exists, it would connect the work of Schmidt et al. with that of Allolio et al. [11], who showed aggregated giant vesicles under influence of $R_{9}$, and would therefore potentially be interesting.

To finish the discussion about dye influence, stacks were again observed with the positively charged Rhodamine-like membrane staining agent Rh18. The dye is directly coupled to an acyl chain, without an intermediate cholesteryl or phosphatidyl moiety. The completely different nature of this dye as opposed to the lipid tail Bodipy dye, serves as strong evidence that the formation of stacks is an intrinsic effect on particular lipid mixtures, independent of the dye used. More rapid growing of tubes (Appendix 11 Figure A 11.1 p. XXXIV) as well as stacks under microscopic illumination was observed with this dye. This might be due to its insertion between the phospholipids, therefore increasing disorder among lipid chains and increase of the area per lipid: these parameters have been correlated to increase of membrane fluidity $[153,189]$.

\section{Lipid and peptide requirements for stack formation}

Stacks were only observed in lipid membranes containing POPG and POPS, and for several peptides. First, the implications of only these lipids showing stack formation will be discussed. Then, the different peptides will be discussed, concluding with an examination of other molecules in literature that have been shown to either cause lamellarisation, fusion or observed stacks.

\section{Lipids}

Out of four lipid mixtures examined, only the ones containing 50\% POPG or $50 \%$ POPS were shown to exhibit stack formation. The assumption that also 50\% POPS membranes exhibited stacks and not a different phenomenon, was validated by similar fluorescence levels to those observed for proven stacks (by AFM imaging) in POPG-containing membranes. It was also established that the dissociation constants for $\mathrm{R}_{6}$ to both these lipid mixtures were similar.

POPS and POPG are similar in size, and both have a net negative charge due to the phosphate group. This was not the case for POPC and POPE, which are 
generally zwitterionic. We suspect that negatively charged lipids are the main drive for stack formation. An approach for further studies could be to investigate whether R-peptides can also form stacks on membranes containing other negatively charged lipids, such as the phosphatidyl inositides (PI) or mostly uncharged glycolipids. Alternatively, stack formation as a function of negative lipid fraction could be explored, analogously to how Robison et al. explored this for the dissociation constants for $R_{8}$ and $K_{9}[36]$.

A further interesting observation is that PG headgroup lipids are mainly found in bacteria. The results of this work therefore seem another manifestation of similar behaviour between antimicrobial and cell-penetrating peptides, which have before been shown to induce both vesicle aggregation and fusion[9]. This is a strong indication that this seemingly fusogenic behaviour of these peptides plays a role in their still controversial mechanisms of entry.

\section{Peptides}

Moving on to the results of different peptides, the most striking observation in this work was that any length R-peptide could induce stack formation, if the concentration was high enough. Per two residues of peptide, a factor 100-10000 increase in concentration was necessary to reliably observe stack formation. The further observation that $\mathrm{K}_{6}$ required a factor 100 increase in concentration with respect to $R_{6}$, is in line with dissociation constants obtained by Robison et al. for $\mathrm{R}_{9}$ vs $\mathrm{K}_{9}[36]$.

As for dissociation constants obtained in this work, they did not exactly overlap with the qualitatively determined threshold concentrations for stack formation: for $R_{6}$, it was higher than expected at nearly $10 \mu \mathrm{M}$ for all lipid mixtures. Furthermore, the difference in dissociation constant with $\mathrm{R}_{4}$ did not increase as strongly as expected from stack formation behaviour: the difference is hardly significant at a factor 3-7 comparted to that for $R_{6}$. Lastly, dissociation constants for $R_{2}$ were ca. 10-20 mM, at which no reproducible stack formation could be observed. We attribute these discrepancies to the qualitative nature of the threshold concentration determination.

However, the trend of higher dissociation constants for shorter R-derivatives, is well-preserved between qualitative and quantitative observations. Lastly, it has to be remarked that the trend observed in this work can well be extrapolated to 
results obtained for $\mathrm{R}_{9}$ on 30\% POPG membranes with a different measurement method: a dissociation constant of $0.029 \mu \mathrm{M}$ was obtained for $\mathrm{R}_{9}$, which differs a factor of ca. 300 from that obtained in this work for $R_{6}$, at ca. 7-8 $\mu \mathrm{M}$.

\section{Membrane Stack formation, fusion \& lamellarisation in literature}

As discussed above, all investigated peptides carrying at least two positively charged groups could form stacks on negatively charged lipid-containing membranes, if peptide concentration was high enough. This raised the question whether there is evidence in literature that this is a generally observed phenomenon for similar conditions: negatively charged lipid bilayers and stackforming or lamellarisation agents that have at least two positive charges, with a linker region of some minimum length. Not only literature mentioning membrane stacks was examined, but also lamellarisation and membrane fusion: all these phenomena involve the screening of membrane charges by oppositely charged agents, hence overcoming inter-bilayer repulsion and allowing to close approach of two lipid bilayers.

There is a plethora of molecules and peptides in literature that can induce either stack formation on model lipid bilayers, or lamellarisation or unspecific fusion[78, 79]. In order to not lose overview, only examples of systems with conditions similar to those in this work will be discussed here: negatively charged lipid membranes bridged by positively charged fusogens or linking agents.

The first example, which has been mentioned multiple times already, is the work of Gräb et al. [170,172] showing stack formation on plant lipid-containing bilayers by long-chain polyamines (LCPAs). LCPAs are amino groups linked by short hydrocarbon chains, generally defined as $\mathrm{CaNb}$, $a$ and $b$ being numeric integers. a signifies the total number of amino groups in the polymer, and b signifies the length of the hydrocarbon chains. The multiple amino groups in LCPAs make for at least two positive charges at neutral $\mathrm{pH}$, at both ends of the molecule. Furthermore, Gräb et al. discovered a dependency on polymer length for stack formation behaviour: the shortest polymer for which stack formation was observed in that work, was C3N7. Two shorter LCPAs did not form stacks, although it was not investigated whether concentration increase would again. A further connection to this work is that stacks were shown to be formed on membranes with only 20 $\%$ POPG present as a negatively charged lipid. It was not investigated whether the 
other employed negatively charged lipid, SQDG, would result in stack formation in the absence of other non-PC headgroup lipids.

Similarly to this work, the next example (Heath et al. [173, 174]) uses POPC:POPG 1:1 or 3:1 lipid mixtures. Here, a conscious choice was made to produce lipid bilayer multi-stacks step by step, with poly-L-lysine (PLL) as a linking agent between the stacks. Lipid vesicles and PLL were alternatingly added to a solid substrate. PLLs of 300,150 and $5 \mathrm{kDa}$ were studied. Their molar concentration equivalents were ca. 0.033-2.0 $\mu \mathrm{M}$ respectively, which is at similar order of magnitude as the $R_{6}$ concentrations used to observe stack formation in this work $(1 \mu \mathrm{M})$. Heath et al. observed that lower $\mathrm{pH}$ and shorter PLLs resulted in more closely packed membrane stacks than high $\mathrm{pH}$ and longer PLLs. The purpose of their work was to create model systems to study the function of various membrane-anchored or transmembrane protein complexes.

As mentioned in the Introduction subchapter 1.3.2 (p. $21 \mathrm{ff}$.), lamellarisation of cell membranes induced by oligoarginines has been observed. For labelled $R_{9}[11]$ as well as $R_{12}$ [32], HeLa cells showed focal highly fluorescent particles on their membrane. Electron microscopy showed that these particles correlated with local highly lamellar structures in the cell membrane, which were not observed without R-peptide[32].

Other R-derivatives than oligoarginines have been reported to induce fusion or stack formation. There are several literature examples of fusogenic[161, 192] or stack formation/lamellarisation [193, 194] behaviour of positively charged peptides in combination with PG headgroup-containing lipid bilayers, or membranes containing a different negatively charged lipid, such as cardiolipin[195] or a PS headgroup $[9,11]$. Interestingly, several of these studies report denaturation or at least structural changes of the peptide upon binding to a lipid bilayer [9, 192], or a decrease in membrane phase transition temperature [193, 194]. Another interesting observation is that these peptides have varying functions, while all having this behaviour in common: one is lysozyme, a short protein found in mammalian saliva which catalyses the hydrolysis of the cell wall of Gram-negative bacteria $[192,195]$. Other antimicrobial peptides studied were three lactoferrin candidacidal peptides[193] and a generally antimicrobial cecropin A-mellitin hybrid[194], Magainin 2, PGLa, MSI-103, Alamethicin and Gramicidin S[9]. Contrastingly, the semen-derived enhancer of viral infection (SEVI) precursor 
PAP $_{248-286}$ dramatically enhances HIV infectivity, but was shown to cause membrane leakage and suspected to cause fusion as well[161].

Wadhwani et al.[9] made a substantial effort to shed light on this seemingly overlapping behaviour of differently membrane-active peptides. They performed fusion assays as well as structural analysis by circular dichroism on multiple antimicrobial (as mentioned above) as well as cell-penetrating peptides (Penetratin, Transportan, MAP, SAP and Pep1) in comparison to HIV fusogenic peptide FP23. Most of these peptides have multiple lysine and/or arginine residues, as well as a net positive charge. They however found that denoted function was not connected to degree of fusogenic behaviour, but rather that artificially designed peptides (MAP, Transportan, Penetratin, MSI-103 and Pep1) were more effective at inducing vesicle aggregation and lipid mixing than naturally occurring peptides. Furthermore, there was a strong correlation between structural change as observed by circular dichroism (CD) spectroscopy, and fusogenic behaviour. They thus demonstrated that positive charge is not necessarily a key factor in causing membrane fusion.

Nevertheless, several research efforts have shown that positively charged ions can cause membrane fusion[79] or stack formation[196, 197], if these membranes contain negatively charged lipids. Cations $\mathrm{Ca}^{2+} \mathrm{Mg}^{2+}, \mathrm{Mn}^{2+}, \mathrm{Zn}^{2+}, \mathrm{La}^{3+}, \mathrm{Sr}^{3+}$ and $\mathrm{H}^{+}$ are positive inorganic ions that have been reported to show fusogenic behaviour, according to a review on fusion induced by small molecules and ions[79]. It mentions multiple studies in which $\mathrm{Ca}^{2+}$ induced fusion in membranes that contained PS-headgroups or sulfatides (headgroups with a sulfate moiety attached)[79]. It has been shown that $\mathrm{Ca}^{2+}$ not only screens the negative charge of the membrane, but also dehydrates the membrane surface. Adams more recently showed that in the presence of $\mathrm{Ca}^{2+}$, the endotoxin lipopolysaccharides (LPS) from Gram-negative bacteria formed stacks in DOPC membranes instead of tubules and holes, thereby illustrating how calcium can mitigate the toxic effect of LPS. Self-assembling stacks have also been reported to be formed by membranes containing a chelating iminodiacetic acid moiety, when copper ions are present to act as a bridge [197].

Taking all these literature findings together, two possible outlooks stemming from this work's results can be anticipated. The first is elaborating on the notion that the guanidine moiety has good potential for further research in the purposeful 
creation of lipid bilayer stacks in order to study membrane proteins, analogous to Heath et al. [173, 174]: bearing in mind that this work and others[36] have directly shown that the guanidine moiety is more effective than the amine moiety found in lysine, molecules bearing multiple guanidine groups could be a good candidate to form more stable systems. Focus would be on the length of hydrocarbon linker between two guanidine moieties, the number of guanidine moieties, and whether branching is necessary and to what degree. Furthermore, it would be interesting to investigate whether simple guanidine-bearing molecules are all disruptive, like N-Propylguanidine investigated in Chapter 4 (p. 61), or whether two or three guanidine moieties per molecule would favour bridging behaviour over disruption. The former seems more likely, since a polyguanidine, polyhexamethylene biguanidine (PHMB), is a widely used antiseptic [198, 199].

The second direction of further work could be to elucidate the relationship between membrane-active peptide function (CPP or AMP) and observed behaviour in model systems. Results in this work and in work of Allolio et al.[11] suggest that additionally to fusion assays, stack formation behaviour on SLBs of different membrane-active peptides could be evaluated, with several different lipid bilayer compositions. Hopefully, this can shed more light on the mechanism of action of these peptides. 



\section{DISCUSSION: CPPS' MECHANISM OF ACTION}

In previous chapters, results on the influence of arginine derivatives and peptides on the physical properties of lipid bilayers were discussed. A guanidine-bearing mimic of the arginine sidechain ( $\mathrm{N}$-propylguanidine, Rside) was shown to make membrane poration in negatively charged lipid bilayers more likely in simulations as well as experiments. This was not found for an oligopeptide with known cellpenetrating capabilities $\left(R_{6}\right)$. However, this peptide did induce stack formation on negatively charged lipid bilayers (containing POPG or POPS) and low concentration. Furthermore, it was shown that most likely any arginine or lysine peptide is able to induce these stacks, if its concentration is high enough. Here, we will discuss what impact these findings have on the understanding and validity of thus far proposed mechanisms of cell entry for CPPs, as an overarching discussion of this work and in an effort at placing this work in context of other CPP-research. For an in-depth discussion and literature comparison of specific experimental findings, we refer to the specific discussions at the ends of Chapter 4 and 5 (Sections 4.6 pp. 86-90 and 5.6 pp. 120-135).

The combination of our findings of stack formation caused by positively charged oligopeptides of likely any length, with the repeated literature mention of lamellarisation, fusion or otherwise membrane interface formation by arginine-rich CPPs or AMPs in literature[9,11,32,161,193,194], indicates that such behaviour is likely an important aspect of arginine-rich CPPs' spontaneous entry into eukaryotic cells. We found that, given the presence of negatively charged lipids in the membrane, likely any length of positively charged peptide can induce membrane lamellarisation at high enough concentration. The observation that 
shortening of the peptides by two residues at a time resulted in a factor 100-1000 increase in dissociation constant and/or lowest concentration for which stack formation was observed, could contribute to an explanation why only pentaarginine[35] or longer R-derivatives[29, 30] have been shown to be CPPs. Furthermore, our results could be extrapolated to several studies, in which a length of 8 residues or longer was reported optimal or necessary for oligopeptides to be active as CPPs $[8,18]$ : the trends in stack formation and dissociation constants we obtained makes it likely that R-peptides longer than six residues will be even more effective at inducing lamellarisation, should this behaviour indeed be proven to be essential for effective CPP entry. Membrane lamellarisation has repeatedly been associated with CPP or AMP activity in literature, not only in model membranes with negatively charged lipids $[11,161,193,194]$, but also in eukaryotic cells [11, 32]. The membranes of eukaryotic cells do not have a high negatively charged lipid headgroup content[200], which further indicates that our stack formation observations on model membranes are part of universal argininerich CPP behaviour. More support for this hypothesis can be found in the ability of arginine-rich CPPs and AMPs to induce fusion and aggregation of model membrane systems with low negatively charged lipid content[9]: membrane fusion and lamellarisation are related, since for both phenomena two or more lipid bilayers are brought closely together, for which inter-bilayer electrostatic repulsion needs to be overcome and water needs to be removed from their contact zone [78, 79, $201,202]$. Further observation of membrane lamellarisation and fusion induced by nona-arginine has recently even led to the proposal of a mechanism of entry specifically involving this lamellarisation: arginine-rich peptides induce lamellarisation, and enter via the formation of a fusion pore (see 1 Introduction Figure 1.4 p. 22) [11].

However, Allolio et al. supported this pore formation aspect of the mechanism by membrane leakage data[11], while data in this work clearly do not support this. There was overall lack of significant membrane leakage over multiple measuring days and with different membrane compositions, despite elevation to high $\mathrm{R}_{6}$ concentrations. This clearly indicates that $R_{6}$ is unable to form significantly stable pores under our experimental conditions. It was noted that literature suggesting a pore formation mechanism of action for membrane-active peptides, does report either vesicle leakage [11] or cell content leakage[203]. Membrane destabilisation could not be observed either in repeated breakthrough force spectroscopy 
experiments, although it could be observed to some degree for $R_{2}$ : breakthrough force in POPG-containing membranes was lowered for $R_{2}$, but for $R_{6}$ it was rather elevated on a stack, compared to the main membrane (Figure 5.2 p. 93).

Further work should deliver more solid proof that a pore formation mechanism for oligo-arginine CPP entry is indeed inadequate in general and not only under experimental conditions used in this work. The first important question is whether a longer R-peptide could reduce breakthrough force and reproducibly show membrane leakage, the last of which was shown in literature[11]. It is possible that a minimum length of peptide is necessary to cause pore formation, analogous to the indication by some studies that a minimum length of oligoarginine peptides ( 8 or 9 residues) is necessary to be an effective CPP $[8,18]$. Studies which suggest lamellarisation as an important aspect of oligoarginine mode of action as a CPP were indeed carried out with longer R-peptides [11, 32]. This can be related to the observation that higher fusogenic activity of membrane-active peptides strongly correlated with changes in secondary structure upon binding to membranes [9]: it has been shown that alpha-helix formation, for example, is likely to require at least seven amino acid residues[204]. Therefore, experiments that can determine whether shorter and longer R-peptides change structure upon interacting with model lipid bilayers, such as CD spectroscopy, would complement this work's results as well. Lastly, demonstration of the same behaviour in more closely biomimetic model membrane systems, such as a lipid composition as used in the fusion assays conducted by Wadhwani et al.[9], would provide even stronger evidence that the observed behaviour of R-peptides in this work relates to general behaviour of arginine-rich CPPs.

If future work indeed shows that longer R-peptides cause membrane destabilisation and leakage, this would suggest that arginine oligopeptides can be divided in two groups, in terms of mode of action: this would confirm that pore formation and at least local and transient membrane destabilisation is part of the spontaneous mechanism of entry of longer CPPs. At the same time, it would give reason to assume that shorter oligo-arginines, without the ability to form pores, are more likely to enter cells by endocytosis: while perhaps not spontaneously entering cells, they have been demonstrated to strongly bind to negative components of the lipid bilayer, leading to accumulation on the membrane surface, which can facilitate cell entry via endocytosis[54, 205]. 
If, however, pore formation and membrane destabilisation cannot be supported for longer R-peptides either under our experimental conditions, this would support the school of thought that actual pore formation cannot be an essential part of the mode of action of CPPs. Pore formation or otherwise membrane destabilisation is likely to lead to cytotoxicity and is thus an overall undesirable behaviour of CPPs[4].

In that case, an adaptation of the mechanism proposed by Allolio et al. [11], so that it does not involve defined pore formation or otherwise destabilisation of the lipid bilayer, would be warranted. Our data suggest that lipid bilayer stacks are connected to the main membrane via multiple stalk-intermediate-like junctions. Moreover, the dynamic behaviour that was observed for lipid bilayer stacks, as well as the proof that external lipid material is brought into the main membrane by fusion, could suggest that continuous formation of such junctions takes place. In fundamental studies on the mechanism of membrane fusion it has been proposed that in the transition from two closely apposed lipid bilayers to a hemifusion stalk intermediate is associated with removal of water from the intermembrane space, but also by temporary destabilisation of the fusing bilayers[79]. These two aspects of the transition to hemifusion stalk could give CPPs the chance to cross membranes during such events, without inducing leakage: the temporary destabilisation of the lipid bilayer would be the moment arginine-rich CPPs 'slip through', while the removal of water from the interbilayer space ensures that no cell or vesicle contents are leaked or exchanged at the same time. An illustration of this idea is given in Figure 6.1.

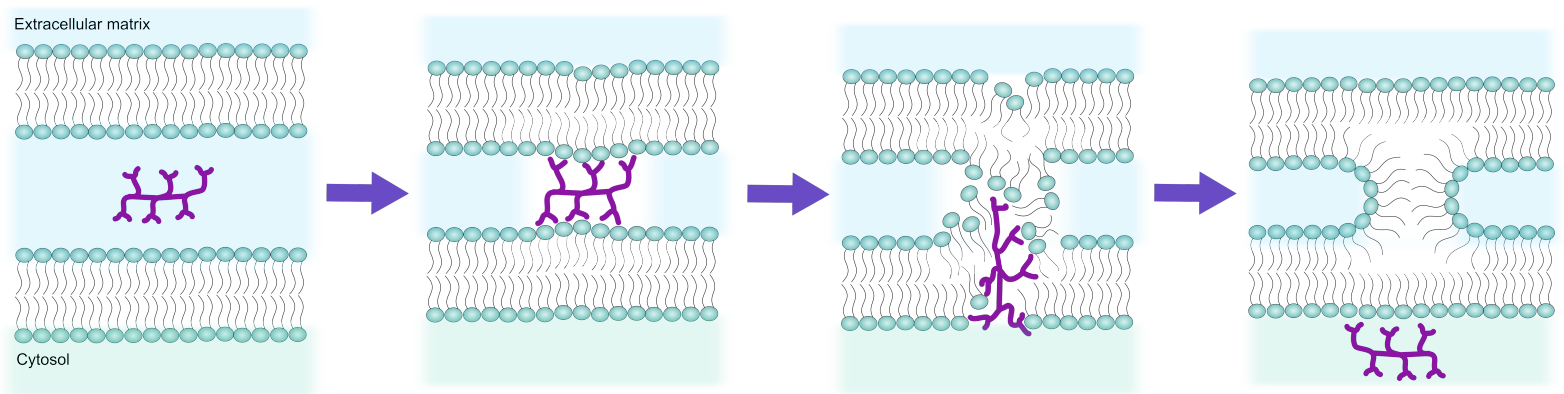

Figure 6.1: Illustration of arginine-rich CPPs entering cells while they facilitate the formation of stalklike junctions between bilayer lamellae. Upon binding of a peptide to both bilayers, water is removed from the bilayer interface (marked as white blur, second illustration from the left). During the destabilisation that precedes the formation of the stalk-like junction in the following picture (for the purpose of illustration, not based on data), the peptide could cross either of the bilayers and could thus enter the cytosol.

There however are several challenges in the way of the validation of this mechanism. The question could be raised whether a mechanism that relies on 
single membrane fusion events would provide sufficient explanation of efficient spontaneous CPP entry. Furthermore, this mechanism would combine the challenging aspects of two widely studied fields (CPPs and membrane fusion): to our knowledge, the details of the transition from membrane docking to $a$ hemifused state cannot be directly observed, but only simulated. Methods like colloidal probe force spectroscopy and optical tweezer experiments can observe removal of water from the intermembrane space and transition to hemifusion[202, 206]. However, they do not provide the details on the behaviour of single molecules that would be necessary for validation of CPP entry during brief transient membrane destabilisation in the transition from membrane docking to hemifusion. Finally, hemifusion is usually defined as only the proximal bilayer leaflets merging[78, 79, 202, 206, 207]. Therefore, temporary destabilisation of the distal leaflets to allow passage of R-peptide during formation of a hemifusion stalk-like structure, could be controversial. 



\section{SUMMARY}

This work intended to shed more light on the intrinsic role of arginine and other basic amino acids in the mechanism of action of CPPs and AMPs. The focus was especially on arginine and its interaction with different lipid headgroups. To explore the role of arginine in pore formation-like mechanisms, simulations (by collaboration partner $\mathrm{Dr}$. Neha Awasthi) as well as breakthrough force spectroscopy and leakage assays were performed with bilayers of varying composition and short arginine derivatives $\mathrm{R}_{\text {side }}$ and $\mathrm{R}_{2}$. Additionally, fluorescence microscopy, fluorescence recovery after photobleaching and reflectometric interference spectroscopy were utilised to characterise binding to and further interactions with model lipid bilayers. Effects of arginine oligopeptides $R_{4}$ and $R_{6}$ as well as oligolysine $\mathrm{K}_{6}$, were explored as well.

In accordance with literature, it was found that all arginine derivatives and peptides tested showed most significant effects on lipid compositions containing negatively charged lipids (POPG and POPS). Rside and to a lesser extent $R_{2}$ increased susceptibility to pore formation according to simulations as well as breakthrough force spectroscopy experiments, at relatively high concentrations. However, this was not found for known CPP $\mathrm{R}_{6}$ : breakthrough force was not reduced, and leakage assays showed no significant leakage. Our data therefore do not support mechanisms of action for CPPs that involve pore formation.

$\mathrm{R}_{6}$ did however induce membrane stack formation on POPG- and POPS-containing solid-supported membranes at low concentration. These stacks form at multiple and sometimes overlapping levels, and based on FRAP data we hypothesize that multiple connections between stacks and main membrane exist. We further hypothesize that the mechanism of formation of membrane stacks is a combination of fusion of external lipid material into the membrane and spreading of excess lipid material stemming from thermal expansion. It is possible that area per lipid 
increase due to R-peptide binding also plays a role, but future work should deliver proof for such a mechanism.

We also found that likely any oligopeptide with multiple guanidine moieties is able to induce stack formation, if its concentration is high enough. Decrease in oligoarginine length by two residues caused the peptide concentration required to induce stack formation to increase by ca. a factor 100 or more. Likewise, an oligolysine pepide $\mathrm{K}_{6}$ required a factor 100 higher concentration to induce stacks than oligo-arginine $R_{6}$.

This trend correlated with the trend observed in dissociation constants determined by RIfS: the shorter the R-peptide, the higher the determined dissociation constant. The trend could be extrapolated to the dissociation constant for $\mathrm{R}_{9}$ on POPG-containing membranes, as given in literature[36]: our determined value for $R_{6}$ exceeded the literature value for $R_{9}$ by ca. a factor 100 .

The observation of membrane stack formation by arginine-rich peptides or otherwise lamellarisation is fairly present in literature. However, our data do not support any proposed mechanism that involves pore formation. We reasoned that possibly, longer arginine-peptides would facilitate pore formation again, likely aided by secondary structure formation. Future studies should address this question.

If future work definitely proves membrane-destabilising capabilities for R-peptides from a certain threshold length, it could be hypothesized that shorter oligoarginines enter cells via endocytosis, while longer ones can also enter spontaneously. This would be due to their pore formation capabilities, which are possibly tied to secondary structure formation.

If on the other hand longer peptides do not destabilise lipid bilayers either under our experimental conditions, this would warrant a revision of thus far proposed mechanisms of cell entry for oligo-arginines. A possible new mechanism of action will then be required to include a role for lamellarisation and fusion of the cell membrane, but should not involve membrane destabilisation or water-permeable pore formation.

In conclusion, this study has provided clear directions for future work, to soon be able to predict oligoarginine CPPs' mechanism of action based on peptide length. 


\section{BIBLIOGRAPHY}

1. Guidotti, G., L. Brambilla, and D. Rossi, Cell-Penetrating Peptides: From Basic Research to Clinics. Trends Pharmacol Sci, 2017. 38(4): p. 406-424.

2. Khalil, I.A. and H. Harashima, An efficient PEGylated gene delivery system with improved targeting: Synergism between octaarginine and a fusogenic peptide. Int. J. Pharm. (Amsterdam, Neth.), 2018. 538(1-2): p. 179-187.

3. Gasparini, G., et al., Cellular uptake: lessons from supramolecular organic chemistry. Chem. Commun. (Cambridge, U. K.), 2015. 51(52): p. 1038910402.

4. Kauffman, W.B., et al., Mechanism Matters: A Taxonomy of Cell Penetrating Peptides. Trends Biochem Sci, 2015. 40(12): p. 749-764.

5. Futaki, S. and I. Nakase, Cell-Surface Interactions on Arginine-Rich CellPenetrating Peptides Allow for Multiplex Modes of Internalization. Acc. Chem. Res., 2017. 50(10): p. 2449-2456.

6. Gestin, M., M. Dowaidar, and Ü. Langel, Uptake Mechanism of CellPenetrating Peptides, in Peptides and Peptide-based Biomaterials and their Biomedical Applications, A. Sunna, A. Care, and P.L. Bergquist, Editors. 2017, Springer International Publishing: Cham. p. 255-264.

7. Ramaker, K., et al., Cell penetrating peptides: a comparative transport analysis for 474 sequence motifs. Drug Deliv, 2018. 25(1): p. 928-937.

8. Wender, P.A., et al., The design, synthesis, and evaluation of molecules that enable or enhance cellular uptake: Peptoid molecular transporters. Proceedings of the National Academy of Sciences, 2000. 97(24): p. 13003-13008.

9. Wadhwani, P., et al., Antimicrobial and cell-penetrating peptides induce lipid vesicle fusion by folding and aggregation. Eur Biophys J, 2012. 41(2): p. 177-87.

10. Herce, H.D., A.E. Garcia, and M.C. Cardoso, Fundamental Molecular Mechanism for the Cellular Uptake of Guanidinium-Rich Molecules. Journal of the American Chemical Society, 2014. 136(50): p. 17459-17467.

11. Allolio, C., et al., Arginine-rich cell-penetrating peptides induce membrane multilamellarity and subsequently enter via formation of a fusion pore. Proceedings of the National Academy of Sciences, 2018. 115(47): p. 11923.

12. Frankel, A.D. and C.O. Pabo, Cellular uptake of the tat protein from human immunodeficiency virus. Cell, 1988. 55(6): p. 1189-1193.

13. Green, M. and P.M. Loewenstein, Autonomous functional domains of chemically synthesized human immunodeficiency virus tat trans-activator protein. Cell, 1988. 55(6): p. 1179-1188.

14. Vives, E., P. Brodin, and B. Lebleu, A truncated HIV-1 Tat protein basic domain rapidly translocates through the plasma membrane and accumulates in the cell nucleus. J Biol Chem, 1997. 272(25): p. 16010-7. 
15. Park, J., et al., Mutational analysis of a human immunodeficiency virus type 1 Tat protein transduction domain which is required for delivery of an exogenous protein into mammalian cells. Journal of General Virology, 2002. 83(5): p. 1173-1181.

16. Joliot, A., et al., Antennapedia homeobox peptide regulates neural morphogenesis. Proceedings of the National Academy of Sciences, 1991. 88(5): p. 1864.

17. Derossi, D., et al., The third helix of the Antennapedia homeodomain translocates through biological membranes. J Biol Chem, 1994. 269(14): p. 10444-50.

18. Mitchell, D.J., et al., Polyarginine enters cells more efficiently than other polycationic homopolymers. J Pept Res, 2000. 56(5): p. 318-25.

19. Ryser, H.J.P. and R. Hancock, Histones and Basic Polyamino Acids Stimulate the Uptake of Albumin by Tumor Cells in Culture. Science, 1965. 150(3695): p. 501.

20. Ryser, H.J.P. and W.-C. Shen, Conjugation of methotrexate to poly(Llysine) increases drug transport and overcomes drug resistance in cultured cells. Proceedings of the National Academy of Sciences, 1978. 75(8): p. 3867.

21. Futaki, S., et al., Arginine-rich peptides and their internalization mechanisms. Biochemical Society Transactions, 2007. 35(4): p. 784.

22. Foerg, C. and H.P. Merkle, On the biomedical promise of cell penetrating peptides: limits versus prospects. J Pharm Sci, 2008. 97(1): p. 144-62.

23. Koren, E. and V.P. Torchilin, Cell-penetrating peptides: breaking through to the other side. Trends in Molecular Medicine, 2012. 18(7): p. 385-393.

24. Milletti, F., Cell-penetrating peptides: classes, origin, and current landscape. Drug Discov Today, 2012. 17(15-16): p. 850-60.

25. Raucher, D. and J.S. Ryu, Cell-penetrating peptides: strategies for anticancer treatment. Trends in Molecular Medicine, 2015. 21(9): p. 560570.

26. Alvaro, I.H., M.T. John, and P. Om, Membrane Interacting Peptides: $A$ Review. Current Protein \& Peptide Science, 2016. 17(8): p. 827-841.

27. Langel, Ü., Methods for Structural Studies of CPPs, in CPP, Cell-Penetrating Peptides. 2019, Springer. p. 289-323.

28. Bechara, C. and S. Sagan, Cell-penetrating peptides: 20 years later, where do we stand? FEBS Letters, 2013. 587(12): p. 1693-1702.

29. Gautam, A., et al., CPPsite: a curated database of cell penetrating peptides. Database : the journal of biological databases and curation, 2012. 2012: p. bas015-bas015.

30. Agrawal, P., et al., CPPsite 2.0: a repository of experimentally validated cell-penetrating peptides. Nucleic Acids Research, 2015. 44(D1): p. D1098-D1103.

31. Jobin, M.L., et al., Biophysical Insight on the Membrane Insertion of an Arginine-Rich Cell-Penetrating Peptide. Int J Mol Sci, 2019. 20(18).

32. Hirose, H., et al., Transient Focal Membrane Deformation Induced by Arginine-rich Peptides Leads to Their Direct Penetration into Cells. Molecular Therapy, 2012. 20(5): p. 984-993.

33. Tünnemann, G., et al., Live-cell analysis of cell penetration ability and toxicity of oligo-arginines. Journal of Peptide Science, 2008. 14(4): p. 469-476.

34. Balhorn, R., et al., Hexa-arginine enhanced uptake and residualization of selective high affinity ligands by Raji lymphoma cells. Molecular cancer, 2009. 8: p. 25-25. 
35. de Cogan, F., et al., Topical Delivery of Anti-VEGF Drugs to the Ocular Posterior Segment Using Cell-Penetrating Peptides. Investigative Ophthalmology \& Visual Science, 2017. 58(5): p. 2578-2590.

36. Robison, A.D., et al., Polyarginine Interacts More Strongly and Cooperatively than Polylysine with Phospholipid Bilayers. J Phys Chem B, 2016. 120(35): p. 9287-96.

37. Buschle, M., et al., Transloading of tumor antigen-derived peptides into antigen-presenting cells. Proceedings of the National Academy of Sciences, 1997. 94(7): p. 3256-3261.

38. Ojeda, P.G., et al., Lysine to arginine mutagenesis of chlorotoxin enhances its cellular uptake. Biopolymers, 2017. 108(5): p. n/a.

39. Stalmans, S., et al., Chemical-functional diversity in cell-penetrating peptides. PLoS One, 2013. 8(8): p. e71752.

40. Thoren, P.E., et al., Membrane binding and translocation of cellpenetrating peptides. Biochemistry, 2004. 43(12): p. 3471-89.

41. Maiolo, J.R., M. Ferrer, and E.A. Ottinger, Effects of cargo molecules on the cellular uptake of arginine-rich cell-penetrating peptides. Biochim Biophys Acta, 2005. 1712(2): p. 161-72.

42. Traboulsi, H., et al., Macrocyclic Cell Penetrating Peptides: A Study of Structure-Penetration Properties. Bioconjugate Chem., 2015. 26(3): p. 405-411.

43. Vasconcelos, L., K. Pärn, and Ü. Langel, Therapeutic potential of cellpenetrating peptides. Therapeutic Delivery, 2013. 4(5): p. 573-591.

44. Kato, T., et al., Plasmid DNA delivery by arginine-rich cell-penetrating peptides containing unnatural amino acids. Bioorg. Med. Chem., 2016. 24(12): p. 2681-2687.

45. Deshpande, P., et al., Transferrin and octaarginine modified dualfunctional liposomes with improved cancer cell targeting and enhanced intracellular delivery for the treatment of ovarian cancer. Drug Delivery, 2018. 25(1): p. 517-532.

46. Habault, J. and J.-L. Poyet, Recent Advances in Cell Penetrating PeptideBased Anticancer Therapies. Molecules (Basel, Switzerland), 2019. 24(5): p. 927.

47. Rothbard, J.B., et al., Conjugation of arginine oligomers to cyclosporin A facilitates topical delivery and inhibition of inflammation. Nat Med, 2000. 6(11): p. 1253-7.

48. Meloni, B.P., et al., Poly-Arginine Peptide-18 (R18) Reduces Brain Injury and Improves Functional Outcomes in a Nonhuman Primate Stroke Model. Neurotherapeutics, 2019.

49. Meloni, B.P., et al., Poly-arginine and arginine-rich peptides are neuroprotective in stroke models. J. Cereb. Blood Flow Metab., 2015. 35(6): p. 993-1004.

50. Lim, J.P. and P.A. Gleeson, Macropinocytosis: an endocytic pathway for internalising large gulps. Immunol Cell Biol, 2011. 89(8): p. 836-43.

51. Nakase, I., et al., Arginine-rich cell-penetrating peptide-modified extracellular vesicles for active macropinocytosis induction and efficient intracellular delivery. Sci. Rep., 2017. 7(1): p. 1-11.

52. Mayor, S. and R.E. Pagano, Pathways of clathrin-independent endocytosis. Nature Reviews Molecular Cell Biology, 2007. 8(8): p. 603-612.

53. Kaksonen, M. and A. Roux, Mechanisms of clathrin-mediated endocytosis. Nature Reviews Molecular Cell Biology, 2018. 19(5): p. 313-326. 
54. Richard, J.P., et al., Cellular uptake of unconjugated TAT peptide involves clathrin-dependent endocytosis and heparan sulfate receptors. Journal of Biological Chemistry, 2005. 280(15): p. 15300-15306.

55. Fuchs, S.M. and R.T. Raines, Pathway for Polyarginine Entry into Mammalian Cells. Biochemistry, 2004. 43(9): p. 2438-2444.

56. Nabi, I.R. and P.U. Le, Caveolae/raft-dependent endocytosis. The Journal of cell biology, 2003. 161(4): p. 673-677.

57. Ferrari, A., et al., Caveolae-mediated internalization of extracellular HIV-1 tat fusion proteins visualized in real time. Molecular therapy, 2003. 8(2): p. 284-294.

58. Kawamoto, S., et al., Inverted micelle formation of cell-penetrating peptide studied by coarse-grained simulation: importance of attractive force between cell-penetrating peptides and lipid head group. J Chem Phys, 2011. 134(9): p. 095103.

59. Derossi, D., et al., Cell Internalization of the Third Helix of the Antennapedia Homeodomain Is Receptor-independent. Journal of Biological Chemistry, 1996. 271(30): p. 18188-18193.

60. Goyal, R.K. and A.K. Mattoo, Plant Antimicrobial Peptides, in Host Defense Peptides and Their Potential as Therapeutic Agents, R.M. Epand, Editor. 2016, Springer International Publishing: Cham. p. 111-136.

61. Wimley, W.C., Describing the Mechanism of Antimicrobial Peptide Action with the Interfacial Activity Model. ACS Chemical Biology, 2010. 5(10): p. 905-917.

62. Henriques, S.T., M.N. Melo, and M.A.R.B. Castanho, Cell-penetrating peptides and antimicrobial peptides: how different are they? The Biochemical journal, 2006. 399(1): p. 1-7.

63. Avci, G.F., B. Sariyar Akbulut, and E. Ozkirimli, Membrane Active Peptides and Their Biophysical Characterization. Biomolecules, 2018. 8(3).

64. Almeida, P.F. and A. Pokorny, Mechanisms of Antimicrobial, Cytolytic, and Cell-Penetrating Peptides: From Kinetics to Thermodynamics.

Biochemistry, 2009. 48(34): p. 8083-8093.

65. Nan, Y.H., et al., Antimicrobial activity, bactericidal mechanism and LPSneutralizing activity of the cell-penetrating peptide pVEC and its analogs. Journal of Peptide Science, 2011. 17(12): p. 812-817.

66. Bahnsen, J.S., et al., Antimicrobial and cell-penetrating properties of penetratin analogs: effect of sequence and secondary structure. Biochimica et Biophysica Acta (BBA)-Biomembranes, 2013. 1828(2): p. 223-232.

67. Splith, K. and I. Neundorf, Antimicrobial peptides with cell-penetrating peptide properties and vice versa. European biophysics journal, 2011. 40(4): p. 387-397.

68. Budagavi, D.P. and A. Chugh, Antibacterial properties of Latarcin 1 derived cell-penetrating peptides. European Journal of Pharmaceutical Sciences, 2018. 115: p. 43-49.

69. Zasloff, M., Magainins, a class of antimicrobial peptides from Xenopus skin: isolation, characterization of two active forms, and partial cDNA sequence of a precursor. Proceedings of the National Academy of Sciences, 1987. 84(15): p. 5449-5453.

70. Bobone, S., et al., The thin line between cell-penetrating and antimicrobial peptides: the case of Pep-1 and Pep-1-K. Journal of Peptide Science, 2011. 17(5): p. 335-341.

71. Kaiser, C.A., et al., Molecular cell biology. 2007: WH Freeman. 
72. Edwards, K.A. and A.J. Baeumner, Liposomes in analyses. Talanta, 2006. 68(5): p. 1421-1431.

73. Akbarzadeh, A., et al., Liposome: classification, preparation, and applications. Nanoscale research letters, 2013. 8(1): p. 102-102.

74. Richter, R.P., R. Bérat, and A.R. Brisson, Formation of Solid-Supported Lipid Bilayers: An Integrated View. Langmuir, 2006. 22(8): p. 3497-3505.

75. Gumí-Audenis, B. and M.I. Giannotti, Structural and Mechanical Characterization of Supported Model Membranes by AFM. 2019: p. 1-27.

76. Hernandez, J.M., et al., Variable cooperativity in SNARE-mediated membrane fusion. Proc Natl Acad Sci U S A, 2014. 111(33): p. 12037-42.

77. Angelova, M.I. and D.S. Dimitrov, Liposome electroformation. Faraday Discussions of the Chemical Society, 1986. 81(0): p. 303-311.

78. Marsden, H.R., I. Tomatsu, and A. Kros, Model systems for membrane fusion. Chemical Society Reviews, 2011. 40(3): p. 1572-1585.

79. Mondal Roy, S. and M. Sarkar, Membrane fusion induced by small molecules and ions. J Lipids, 2011. 2011: p. 528784.

80. Smolarsky, M., et al., A simple fluorescent method to determine complement-mediated liposome immune lysis. Journal of immunological methods, 1977. 15(3): p. 255-265.

81. Allen, T. and L. Cleland, Serum-induced leakage of liposome contents. Biochimica et Biophysica Acta (BBA)-Biomembranes, 1980. 597(2): p. 418-426.

82. Weinstein, J.N., et al., Liposome-cell interaction: transfer and intracellular release of a trapped fluorescent marker. Science, 1977. 195(4277): p. 489-92.

83. Rule, G.S., R.A. Montagna, and R.A. Durst, Characteristics of DNA-tagged liposomes allowing their use in capillary-migration, sandwich-hybridization assays. Analytical biochemistry, 1997. 244(2): p. 260-269.

84. McCabe, I.P. and M.B. Forstner, Polymer Supported Lipid Bilayers. Open Journal of Biophysics, 2013. 03(01): p. 59-69.

85. Hennesthal, C. and C. Steinem, Pore-spanning lipid bilayers visualized by scanning force microscopy. Journal of the American Chemical Society, 2000. 122(33): p. 8085-8086.

86. Mey, I., et al., Local Membrane Mechanics of Pore-Spanning Bilayers. Journal of the American Chemical Society, 2009. 131(20): p. 7031-7039.

87. Kocun, M. and A. Janshoff, Pulling tethers from pore-spanning bilayers: towards simultaneous determination of local bending modulus and lateral tension of membranes. Small, 2012. 8(6): p. 847-51.

88. Faysal, K.R., et al., Lipid bilayers are long-lived on solvent cleaned plasma-oxidized poly (dimethyl) siloxane (ox-PDMS). PloS one, 2017. 12(1): p. e0169487.

89. Kliesch, T.-T., et al., Membrane tension increases fusion efficiency of model membranes in the presence of SNAREs. Scientific reports, 2017. 7(1): p. 12070.

90. Hsieh, W.-T., et al., Curvature Sorting of Peripheral Proteins on SolidSupported Wavy Membranes. Langmuir, 2012. 28(35): p. 12838-12843.

91. Savić, F., et al., Geometry of the Contact Zone between Fused MembraneCoated Beads Mimicking Cell-Cell Fusion. Biophysical Journal, 2016. 110(10): p. 2216-2228.

92. Bao, C., et al., Optical Fusion Assay Based on Membrane-Coated Spheres in a 2D Assembly. Journal of the American Chemical Society, 2013. 135(33): p. 12176-12179. 
93. Blodgett, K.B., Films Built by Depositing Successive Monomolecular Layers on a Solid Surface. Journal of the American Chemical Society, 1935. 57(6): p. 1007-1022.

94. Gumí-Audenis, B. and M.I. Giannotti, Structural and Mechanical Characterization of Supported Model Membranes by AFM, in Biomimetic Lipid Membranes: Fundamentals, Applications, and Commercialization. 2019, Springer. p. 1-27.

95. Mingeot-Leclercq, M.-P., et al., Atomic force microscopy of supported lipid bilayers. Nature protocols, 2008. 3(10): p. 1654-1659.

96. Mennicke, U. and T. Salditt, Preparation of Solid-Supported Lipid Bilayers by Spin-Coating. Langmuir, 2002. 18(21): p. 8172-8177.

97. Batatolli, A.C.S.L.A., Structure of Spin-Coated Lipid Films and Domain Formation in Supported Membranes Formed by Hydration. Langmuir, 2004. 20(22): p. 9720-9728.

98. Binnig, G., C.F. Quate, and C. Gerber, Atomic Force Microscope. Physical Review Letters, 1986. 56(9): p. 930-933.

99. Eaton, P. and P. West, Atomic Force Microscopy. 2010: Oxford University Press.

100. Braga, P.C. and D. Ricci, Atomic Force Microscopy in Biomedical Research: Methods and Protocols. 2011: Humana Press.

101. Azeloglu, E.U. and K.D. Costa, Atomic Force Microscopy in Mechanobiology: Measuring Microelastic Heterogeneity of Living Cells, in Atomic Force Microscopy in Biomedical Research: Methods and Protocols, P.C. Braga and D. Ricci, Editors. 2011, Humana Press: Totowa, NJ. p. 303329.

102. Benoit, M. and C. Selhuber-Unkel, Measuring Cell Adhesion Forces: Theory and Principles, in Atomic Force Microscopy in Biomedical Research:

Methods and Protocols, P.C. Braga and D. Ricci, Editors. 2011, Humana Press: Totowa, NJ. p. 355-377.

103. Karsch, S., et al., An intact keratin network is crucial for mechanical integrity and barrier function in keratinocyte cell sheets. Cellular and Molecular Life Sciences, 2020.

104. Garcia-Manyes, S., G. Oncins, and F. Sanz, Effect of temperature on the nanomechanics of lipid bilayers studied by force spectroscopy. Biophys J, 2005. 89(6): p. 4261-74.

105. Schuy, S. and A. Janshoff, Thermal expansion of microstructured DMPC bilayers quantified by temperature-controlled atomic force microscopy. Chemphyschem, 2006. 7(6): p. 1207-10.

106. Alessandrini, A., et al., Dynamic Force Spectroscopy on Supported Lipid Bilayers: Effect of Temperature and Sample Preparation. Biophysical Journal, 2012. 103(1): p. 38-47.

107. Garcia-Manyes, S., G. Oncins, and F. Sanz, Effect of ion-binding and chemical phospholipid structure on the nanomechanics of lipid bilayers studied by force spectroscopy. Biophys J, 2005. 89(3): p. 1812-26.

108. Martin-Molina, A., C. Rodriguez-Beas, and J. Faraudo, Effect of calcium and magnesium on phosphatidy/serine membranes: experiments and allatomic simulations. Biophys J, 2012. 102(9): p. 2095-103.

109. Redondo-Morata, L., G. Oncins, and F. Sanz, Force spectroscopy reveals the effect of different ions in the nanomechanical behavior of phospholipid model membranes: the case of potassium cation. Biophys J, 2012. 102(1): p. 66-74.

110. Piantanida, L., et al., Ions Modulate Stress-Induced Nanotexture in Supported Fluid Lipid Bilayers. Biophys J, 2017. 113(2): p. 426-439. 
111. Leivers, M., et al., Measurement of Forces between Supported Cationic Bilayers by Colloid Probe Atomic Force Microscopy: Electrolyte Concentration and Composition. Langmuir, 2019. 35(3): p. 729-738.

112. Vinogradova, O.I., Slippage of water over hydrophobic surfaces. International journal of mineral processing, 1999. 56(1-4): p. 31-60.

113. Alcaraz, J., et al., Correction of Microrheological Measurements of Soft Samples with Atomic Force Microscopy for the Hydrodynamic Drag on the Cantilever. Langmuir, 2002. 18(3): p. 716-721.

114. Sader, J.E., J.W. Chon, and P. Mulvaney, Calibration of rectangular atomic force microscope cantilevers. Review of scientific instruments, 1999. 70(10): p. 3967-3969.

115. Butt, H.-J., B. Cappella, and M. Kappl, Force measurements with the atomic force microscope: Technique, interpretation and applications. Surface Science Reports, 2005. 59(1): p. 1-152.

116. Hutter, J.L. and J. Bechhoefer, Calibration of atomic-force microscope tips. Review of Scientific Instruments, 1993. 64(7): p. 1868-1873.

117. Butt, H.-J. and M. Jaschke, Calculation of thermal noise in atomic force microscopy. Nanotechnology, 1995. 6(1): p. 1.

118. Ueber die Berührung fester elastischer Körper, in Journal für die reine und angewandte Mathematik (Crelles Journal). 1882. p. 156.

119. Johnson, K.L., et al., Surface energy and the contact of elastic solids. Proceedings of the Royal Society of London. A. Mathematical and Physical Sciences, 1971. 324(1558): p. 301-313.

120. Derjaguin, B.V., V.M. Muller, and Y.P. Toporov, Effect of contact deformations on the adhesion of particles. Journal of Colloid and Interface Science, 1975. 53(2): p. 314-326.

121. Muller, V.M., V.S. Yushchenko, and B.V. Derjaguin, On the influence of molecular forces on the deformation of an elastic sphere and its sticking to a rigid plane. Journal of Colloid and Interface Science, 1980. 77(1): p. 91101.

122. Muller, V.M., B.V. Derjaguin, and Y.P. Toporov, On two methods of calculation of the force of sticking of an elastic sphere to a rigid plane. Colloids and Surfaces, 1983. 7(3): p. 251-259.

123. Saavedra V, O., et al., Compression, Rupture, and Puncture of Model Membranes at the Molecular Scale. Langmuir, 2020. 36(21): p. 57095716.

124. Butt, H.J. and V. Franz, Rupture of molecular thin films observed in atomic force microscopy. I. Theory. Phys Rev E Stat Nonlin Soft Matter Phys, 2002. 66(3 Pt 1): p. 031601.

125. Loi, S., et al., Rupture of molecular thin films observed in atomic force microscopy. II. Experiment. Phys Rev E Stat Nonlin Soft Matter Phys, 2002. 66(3 Pt 1): p. 031602.

126. Franz, V., et al., Tip penetration through lipid bilayers in atomic force microscopy. Colloids and Surfaces B-Biointerfaces, v.23, 191-200 (2002), 2002. 23.

127. Künneke, S., D. Krüger, and A. Janshoff, Scrutiny of the Failure of Lipid Membranes as a Function of Headgroups, Chain Length, and Lamellarity Measured by Scanning Force Microscopy. Biophysical Journal, 2004. 86(3): p. 1545-1553.

128. Gauglitz, G., Direct optical sensors: principles and selected applications. Anal Bioanal Chem, 2005. 381(1): p. 141-55. 
129. Gauglitz, G., et al., Chemical and biochemical sensors based on interferometry at thin (multi-) layers. Sensors and Actuators B: Chemical, 1993. 11(1-3): p. 21-27.

130. Heavens, O.S., Optical properties of thin films. Reports on Progress in Physics, 1960. 23(1): p. 1-65.

131. Stephan, M., et al., Binding assay for low molecular weight analytes based on reflectometry of absorbing molecules in porous substrates. Analyst, 2014. 139(8): p. 1987-1992.

132. Stephan, M., et al., Combining reflectometry and fluorescence microscopy: an assay for the investigation of leakage processes across lipid membranes. Anal Chem, 2014. 86(3): p. 1366-71.

133. Murphy, D.B., Fluorescence microscopy, in Fundamentals of light microscopy and electronic imaging. 2001, John Wiley \& Sons.

134. Murphy, D.B., Confocal laser Icanning microscopy, in Fundamentals of light microscopy and electronic imaging. 2001, John Wiley \& Sons. p. 205-232.

135. Diaspro, A., et al., Multi-photon excitation microscopy. BioMedical Engineering OnLine, 2006. 5(1): p. 36.

136. Awasthi, N. and J.S. Hub, Simulations of Pore Formation in Lipid Membranes: Reaction Coordinates, Convergence, Hysteresis, and FiniteSize Effects. J Chem Theory Comput, 2016. 12(7): p. 3261-9.

137. Hub, J.S. and N. Awasthi, Probing a Continuous Polar Defect: A Reaction Coordinate for Pore Formation in Lipid Membranes. J Chem Theory Comput, 2017. 13(5): p. 2352-2366.

138. Awasthi, N. and J. Hub, Free-Energy Calculations of Pore Formation in Lipid Membranes. Biomembrane Simulations: Computational Studies of Biological Membranes, 2019: p. 109.

139. Mirjalili, V. and M. Feig, Density-biased sampling: a robust computational method for studying pore formation in membranes. Journal of chemical theory and computation, 2014. 11(1): p. 343-350.

140. Roux, B., The calculation of the potential of mean force using computer simulations. Computer Physics Communications, 1995. 91(1): p. 275-282.

141. Hess, B., et al., GROMACS 4: Algorithms for Highly Efficient, LoadBalanced, and Scalable Molecular Simulation. Journal of Chemical Theory and Computation, 2008. 4(3): p. 435-447.

142. Nečas, D. and P. Klapetek, Gwyddion: an open-source software for SPM data analysis, in Open Physics. 2012. p. 181.

143. Stephan, M., et al., Binding assay for low molecular weight analytes based on reflectometry of absorbing molecules in porous substrates. Analyst, 2014. 139(8): p. 1987-92.

144. Butt, H.J., K. Graf, and M. Kappl, Adsorption, in Physics and Chemistry of Interfaces. 2003, Wiley-VCH Verlag GmbH \& Co. KGaA. p. 177-205.

145. Gesztelyi, R., et al., The Hill equation and the origin of quantitative pharmacology. Archive for History of Exact Sciences, 2012. 66(4): p. 427438.

146. Stefan, M.I. and N. Le Novère, Cooperative binding. PLoS computational biology, 2013. 9(6): p. e1003106-e1003106.

147. Moré, J.J., The Levenberg-Marquardt algorithm: implementation and theory, in Numerical analysis. 1978, Springer. p. 105-116.

148. Schindelin, J., et al., Fiji: an open-source platform for biological-image analysis. Nature Methods, 2012. 9(7): p. 676-682.

149. Jonsson, P., et al., A method improving the accuracy of fluorescence recovery after photobleaching analysis. Biophys J, 2008. 95(11): p. 533448. 
150. Savić, F., Theorie \& Design von FRAP-Experimenten auf komplexen Geometrien. 2013: Göttingen.

151. Morandat, S., et al., Atomic force microscopy of model lipid membranes. Anal Bioanal Chem, 2013. 405(5): p. 1445-61.

152. Janmey, P.A. and P.K.J. Kinnunen, Biophysical properties of lipids and dynamic membranes. Trends in Cell Biology, 2006. 16(10): p. 538-546.

153. Dickey, A. and R. Faller, Examining the contributions of lipid shape and headgroup charge on bilayer behavior. Biophys J, 2008. 95(6): p. 263646.

154. Seiwert, D., et al., The Nonbilayer Lipid MGDG and the Major LightHarvesting Complex (LHCII) Promote Membrane Stacking in Supported Lipid Bilayers. Biochemistry, 2018. 57(15): p. 2278-2288.

155. Milovanovic, D., et al., Hydrophobic mismatch sorts SNARE proteins into distinct membrane domains. Nature Communications, 2015. 6(1): p. 5984.

156. Schuy, S., et al., Structure and thermotropic phase behavior of fluorinated phospholipid bilayers: a combined attenuated total reflection FTIR spectroscopy and imaging ellipsometry study. The journal of physical chemistry. B, 2008. 112(28): p. 8250-8256.

157. Faiss, S., et al., Phase Transition of Individually Addressable Microstructured Membranes Visualized by Imaging Ellipsometry. The Journal of Physical Chemistry B, 2007. 111(50): p. 13979-13986.

158. Böckmann, R.A., et al., Effect of Sodium Chloride on a Lipid Bilayer. Biophysical Journal, 2003. 85(3): p. 1647-1655.

159. Böckmann, R.A. and H. Grubmüller, Multistep Binding of Divalent Cations to Phospholipid Bilayers: A Molecular Dynamics Study. Angewandte Chemie International Edition, 2004. 43(8): p. 1021-1024.

160. Ladokhin, A.S., W.C. Wimley, and S.H. White, Leakage of membrane vesicle contents: determination of mechanism using fluorescence requenching. Biophysical Journal, 1995. 69(5): p. 1964-1971.

161. Brender, J., et al., The Sevi Precursor Peptide PAP248-286, a Dramatic Enhancer of HIV Infectivity, Promotes Lipid Aggregation and Fusion. Biophysical Journal, 2010. 98.

162. Paul, A., et al., Inhibition of Alzheimer's Amyloid- $\beta$ Peptide Aggregation and its Disruption by a Conformationally Restricted $a / \beta$ Hybrid Peptide. Chem. Commun., 2014. 51.

163. Sorochkina, A.I., et al., Peptide-induced membrane leakage by lysine derivatives of gramicidin A in liposomes, planar bilayers, and erythrocytes. Biochim Biophys Acta, 2013. 1828(11): p. 2428-35.

164. Bakonyi, M., et al., Stratum corneum lipid liposomes for investigating skin penetration enhancer effects. RSC Advances, 2018. 8(48): p. 2746427469.

165. Baumgart, T., et al., Fluorescence probe partitioning between Lo/Ld phases in lipid membranes. Biochimica et biophysica acta, 2007. 1768(9): p. 2182-2194.

166. Caffrey, M. and J.P. Hogan, LIPIDAT: a database of lipid phase transition temperatures and enthalpy changes. DMPC data subset analysis. Chemistry and physics of lipids, 1992. 61 1: p. 1-109.

167. Garcia-Manyes, S. and F. Sanz, Nanomechanics of lipid bilayers by force spectroscopy with AFM: a perspective. Biochim Biophys Acta, 2010.

1798(4): p. 741-9. 
168. Gedig, M., S. Faiss, and A. Janshoff, Melting and interdigitation of microstructured solid supported membranes quantified by imaging ellipsometry. Biointerphases, 2008. 3(2): p. Fa51.

169. Connell, S.D. and D.A. Smith, The atomic force microscope as a tool for studying phase separation in lipid membranes (Review). Molecular Membrane Biology, 2006. 23(1): p. 17-28.

170. Grab, O., et al., 3D-Membrane Stacks on Supported Membranes Composed of Diatom Lipids Induced by Long-Chain Polyamines. Langmuir, 2016. 32(39): p. 10144-10152.

171. Jing, B., et al., Polyoxometalate macroion induced phase and morphology instability of lipid membrane. Chemical Science, 2013. 4(10): p. 3818-3826.

172. Gräb, O., Solid Supported Model Membranes Containing Plant Glycolipids: A Tool to Study Interactions between Diatom Biomolecules and the Silicalemma in vitro, in Institute of Organic and Biomolecular Chemistry. 2017, University of Göttingen: Göttingen. p. 214.

173. Heath, G.R., et al., Layer-by-Layer Assembly of Supported Lipid Bilayer Poly-l-Lysine Multilayers. Biomacromolecules, 2016. 17(1): p. 324-335.

174. Heath, G.R., et al., Multilayered Lipid Membrane Stacks for Biocatalysis Using Membrane Enzymes. Advanced Functional Materials, 2017. 27(17): p. 1606265.

175. Zhao, H., P.H. Brown, and P. Schuck, On the distribution of protein refractive index increments. Biophysical journal, 2011. 100(9): p. 2309-2317.

176. Axelrod, D., et al., Mobility measurement by analysis of fluorescence photobleaching recovery kinetics. Biophysical Journal, 1976. 16(9): p. 1055-1069.

177. Fick, A., Ueber Diffusion. Annalen der Physik, 1855. 170(1): p. 59-86.

178. Rawicz, W., et al., Elasticity, strength, and water permeability of bilayers that contain raft microdomain-forming lipids. Biophys J, 2008. 94(12): p. 4725-36.

179. Chaudhury, M.K. and S. Ohki, Correlation between membrane expansion and temperature-induced membrane fusion. Biochimica et Biophysica Acta (BBA) - Biomembranes, 1981. 642(2): p. 365-374.

180. Shimomura, M. and T. Kunitake, Fusion and phase separation of ammonium bilayer membranes. Chemistry Letters, 1981. 10(7): p. 1001-1004.

181. Sun, S.T., E.P. Day, and J.T. Ho, Temperature dependence of calciuminduced fusion of sonicated phosphatidylserine vesicles. Proceedings of the National Academy of Sciences, 1978. 75(9): p. 4325.

182. Reimhult, E., F. Höök, and B. Kasemo, Temperature dependence of formation of a supported phospholipid bilayer from vesicles on \$\{lmathrm\{SiO\}\}_\{2\}\$. Physical Review E, 2002. 66(5): p. 051905.

183. Staykova, M., et al., Mechanics of surface area regulation in cells examined with confined lipid membranes. Proc Natl Acad Sci U S A, 2011. 108(22): p. 9084-8.

184. Klymchenko, Andrey S. and R. Kreder, Fluorescent Probes for Lipid Rafts: From Model Membranes to Living Cells. Chemistry \& Biology, 2014. 21(1): p. 97-113.

185. P. Gorbenko, G., N. O. Mchedlov-Petrossyan, and T. A. Chernaya, Ionic equilibria in microheterogeneous systems Protolytic behaviour of indicator dyes in mixed phosphatidylcholine-diphosphatidylglycerol liposomes. 
Journal of the Chemical Society, Faraday Transactions, 1998. 94(15): p. 2117-2125.

186. Filippov, A., G. Orädd, and G. Lindblom, The Effect of Cholesterol on the Lateral Diffusion of Phospholipids in Oriented Bilayers. Biophysical Journal, 2003. 84(5): p. 3079-3086.

187. Jan Akhunzada, M., et al., Interplay between lipid lateral diffusion, dye concentration and membrane permeability unveiled by a combined spectroscopic and computational study of a model lipid bilayer. Scientific Reports, 2019. 9(1): p. 1508.

188. Daniels, C.R., C. Reznik, and C.F. Landes, Dye Diffusion at Surfaces: Charge Matters. Langmuir, 2010. 26(7): p. 4807-4812.

189. Blom, H., et al., Electrostatic interactions of fluorescent molecules with dielectric interfaces studied by total internal reflection fluorescence correlation spectroscopy. International journal of molecular sciences, 2010. 11(2): p. 386-406.

190. Puu, G. and I. Gustafson, Planar lipid bilayers on solid supports from liposomes - factors of importance for kinetics and stability. Biochimica et Biophysica Acta (BBA) - Biomembranes, 1997. 1327(2): p. 149-161.

191. Schmid, E.M., et al., Size-dependent protein segregation at membrane interfaces. Nature physics, 2016. 12(7): p. 704-711.

192. Al Kayal, T., et al., Lysozyme interaction with negatively charged lipid bilayers: protein aggregation and membrane fusion. Soft Matter, 2012. 8(16): p. 4524-4534.

193. Silva, T., et al., Structural diversity and mode of action on lipid membranes of three lactoferrin candidacidal peptides. Biochimica et Biophysica Acta (BBA) - Biomembranes, 2013. 1828(5): p. 1329-1339.

194. Silva, T., et al., Unravelling a Mechanism of Action for a Cecropin AMelittin Hybrid Antimicrobial Peptide: The Induced Formation of Multilamellar Lipid Stacks. Langmuir, 2018. 34(5): p. 2158-2170.

195. Trusova, V.M., et al., Morphological changes of supported lipid bilayers induced by lysozyme: Planar domain formation vs. multilayer stacking. Colloids Surf., B, 2010. 80(2): p. 219-226.

196. Adams, Peter G., et al., Lipopolysaccharide-Induced Dynamic Lipid Membrane Reorganization: Tubules, Perforations, and Stacks. Biophysical Journal, 2014. 106(11): p. 2395-2407.

197. Waggoner, T.A., et al., Self-Assembled Columns of Stacked Lipid Bilayers Mediated by Metal Ion Recognition. Journal of the American Chemical Society, 2001. 123(3): p. 496-497.

198. To, E., et al., The Effectiveness of Topical Polyhexamethylene Biguanide (PHMB) Agents for the Treatment of Chronic Wounds: A Systematic Review. Surgical technology international, 2016. XXVIII.

199. Fjeld, H. and E. Lingaas, Polyhexanide - Safety and efficacy as an antiseptic. Tidsskrift for den Norske laegeforening : tidsskrift for praktisk medicin, ny raekke, 2016. 136: p. 707-711.

200. van Meer, G., D.R. Voelker, and G.W. Feigenson, Membrane lipids: where they are and how they behave. Nat Rev Mol Cell Biol, 2008. 9(2): p. 11224.

201. Chernomordik, L.V. and M.M. Kozlov, Mechanics of membrane fusion. Nature Structural \& Molecular Biology, 2008. 15(7): p. 675-683.

202. Oelkers, M., et al., SNARE-mediated membrane fusion trajectories derived from force-clamp experiments. Proceedings of the National Academy of Sciences, 2016. 113(46): p. 13051. 
203. Herce, H.D., et al., Arginine-rich peptides destabilize the plasma membrane, consistent with a pore formation translocation mechanism of cell-penetrating peptides. Biophys J, 2009. 97(7): p. 1917-25.

204. Manning, M.C., M. Illangasekare, and R.W. Woody, Circular dichroism studies of distorted alpha-helices, twisted beta-sheets, and beta turns. Biophys Chem, 1988. 31(1-2): p. 77-86.

205. Jiao, C.Y., et al., Translocation and endocytosis for cell-penetrating peptide internalization. J Biol Chem, 2009. 284(49): p. 33957-65.

206. Keidel, A., T.F. Bartsch, and E.-L. Florin, Direct observation of intermediate states in model membrane fusion. Scientific Reports, 2016. 6: p. 23691.

207. Otterstrom, J. and A.M. van Oijen, Visualization of Membrane Fusion, One Particle at a Time. Biochemistry, 2013. 52(10): p. 1654-1668. 


\section{APPENDIX}

\section{Supplementary data}

\section{Shift of breakthrough force without significant change of experimental conditions}

Reference link: $[107,158]$

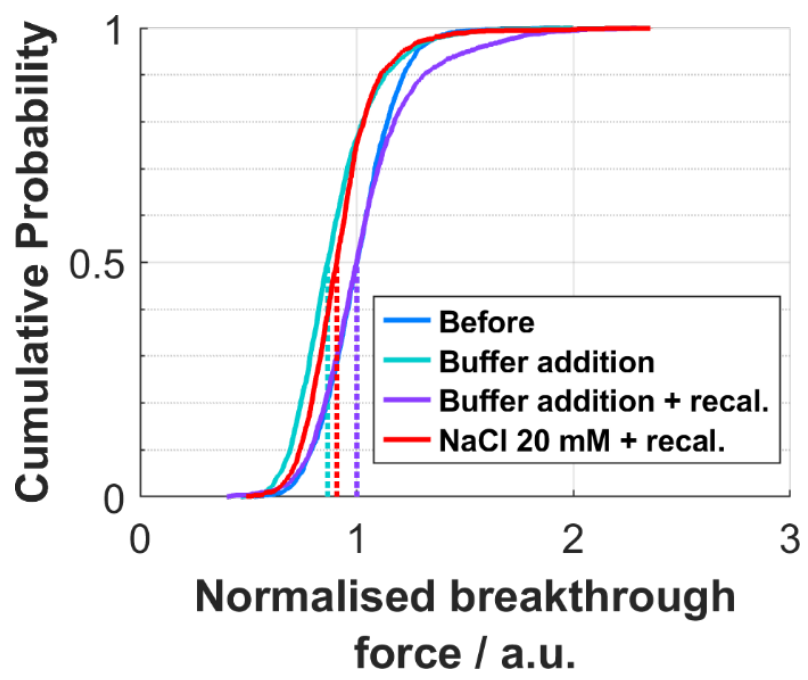

\begin{tabular}{|r|c|c|}
\hline Before & $\begin{array}{c}\text { Mean } \pm \text { St. } \\
\text { deviation }\end{array}$ & Median \\
\hline Buffer addn. & $0.01 \pm 0.17$ & 1.00 \\
\hline Buffer addn. + & $1.03 \pm 0.19$ & 0.87 \\
\hline recal. & 1.03 & 1.00 \\
\hline $\mathbf{N a C l} 20$ mM + & $0.92 \pm 0.18$ & 0.91 \\
\hline recal.
\end{tabular}

Figure A 1.1: Cumulative plots of normalised experimental data to examine to what extent data distributions can shift without intentionally changing the conditions. The $X$-axis represents the values measured, the $\mathrm{Y}$-axis shows the fraction of data at or below each $\mathrm{X}$-value. The part of the curve with the largest slope would be the peak in a histogram. Medians corresponding to this peak, and to $50 \%$ of the data, are indicated as dashed lines. First, a small volume of measuring buffer was added before collecting force curves again. Even though this should not lead to a change in conditions, a $13 \%$ shift in median was observed for the breakthrough forces (cyan curve). Recalibration of the cantilever largely corrected this shift (purple curve), although the distribution showed an additional tail to higher forces. Addition of $20 \mathrm{mM} \mathrm{NaCl}$ again caused a downward shift of about $10 \%$ despite recalibration, while the expectation was that an upwards shift should occur in this case, if any, since this was demonstrated in literature for ions at different concentrations $[107,158]$. 
2. Individual breakthrough force spectroscopy experiments for POPC:BP 99:1

A

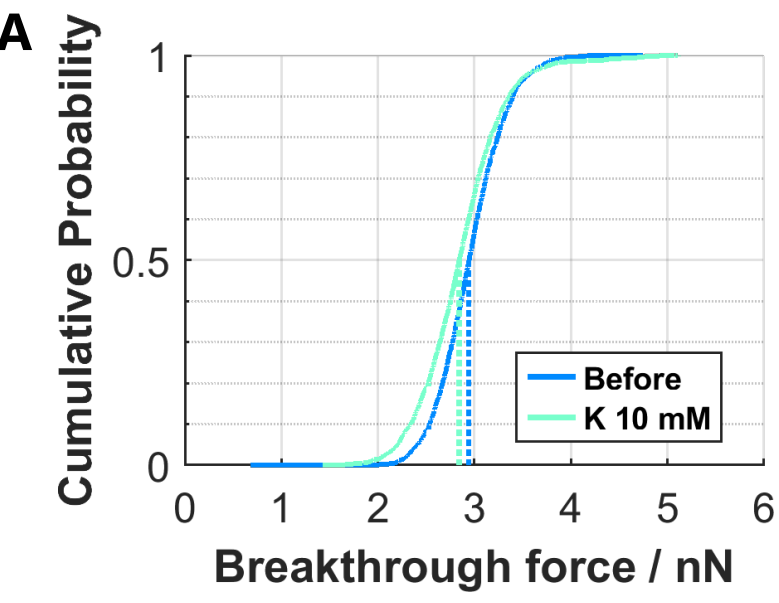

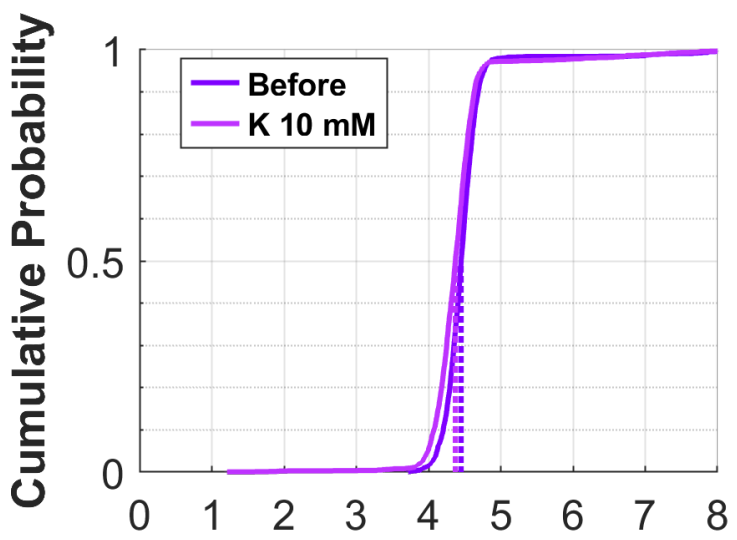

Breakthrough distance / $\mathrm{nm}$
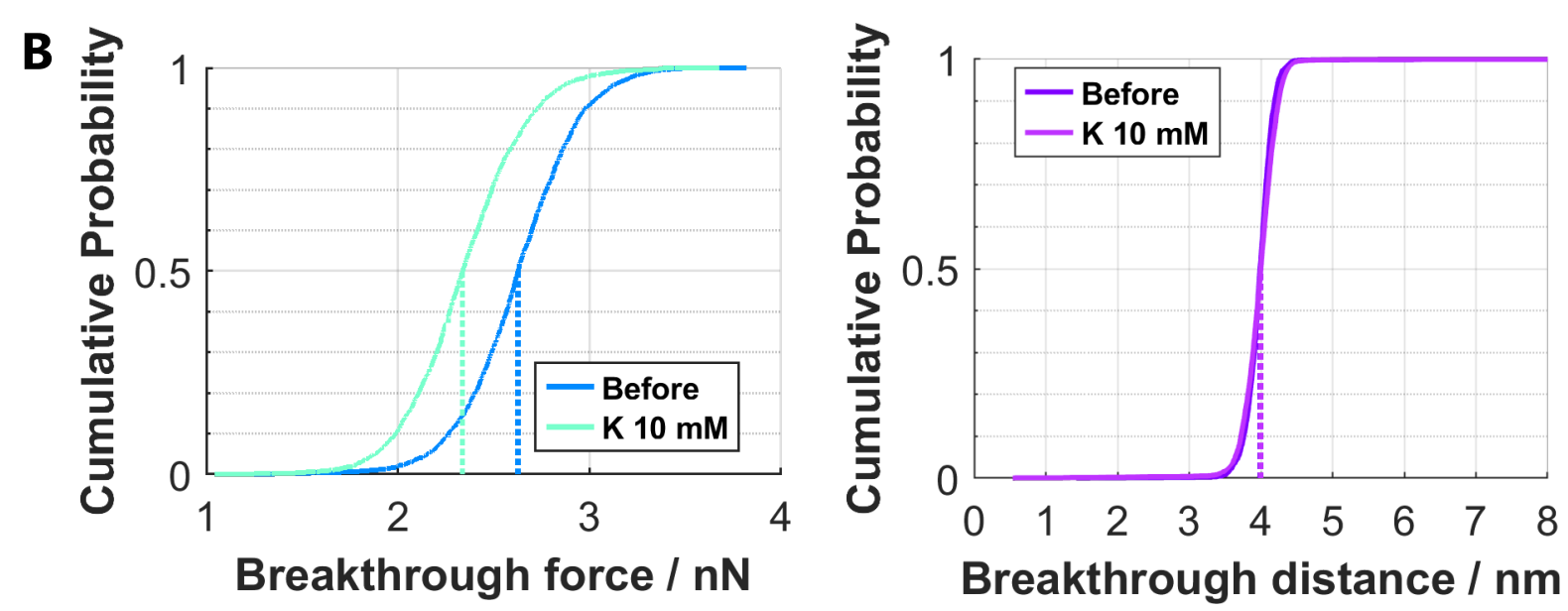

Figure A 1.1: Results of individual breakthrough force spectroscopy experiments on POPC:BP 99:1 membranes with lysine $(K)$. The left plots represent breakthrough force results, the right plots show breakthrough distance results. The part of the curves with the largest slope would be the peak in a histogram. Medians corresponding to this peak are indicated as dashed lines. A: Experiment 1; B: Experiment 2.

Table A 2.1: Means, medians and standard deviations for all breakthrough force spectroscopy experiments on a POPC:BP 99:1 lipid composition, with lysine (K), as shown in Figure A 2.1.

\section{Breakthrough Force}

Before
K $10 \mathrm{mM}$

\section{Breakthrough Distance}

Before

K $10 \mathrm{mM}$

\begin{tabular}{|c|c|c|c|c|c|c|c|c|}
\hline Exp. 1 & Absolute / $\mathrm{nN}$ & $\begin{array}{l}\text { Normalised / } \\
\text { a.u. }\end{array}$ & Absolute / $\mathrm{nN}$ & $\begin{array}{l}\text { Normalised / } \\
\text { a.u. }\end{array}$ & Absolute / nm & $\begin{array}{c}\text { Normalised / } \\
\text { a.u. }\end{array}$ & Absolute / nm & $\begin{array}{l}\text { Normalised / } \\
\text { a.u. }\end{array}$ \\
\hline $\begin{array}{r}\text { Mean } \pm \text { St } \\
\text { deviation }\end{array}$ & $\begin{array}{c}2.96 \pm \\
0.35\end{array}$ & $\begin{array}{c}1.00 \pm \\
0.12\end{array}$ & $\begin{array}{c}2.86 \pm \\
0.44\end{array}$ & $\begin{array}{c}0.97 \pm \\
0.15\end{array}$ & $\begin{array}{c}4.49 \pm \\
0.44\end{array}$ & $\begin{array}{c}1.01 \pm \\
0.10\end{array}$ & $\begin{array}{c}4.43 \pm \\
0.81\end{array}$ & $\begin{array}{c}1.00 \pm \\
0.18\end{array}$ \\
\hline Median & 2.94 & 1.00 & 2.84 & 0.97 & 4.45 & 1.00 & 4.37 & 0.98 \\
\hline \multicolumn{9}{|l|}{ Exp. 2} \\
\hline $\begin{array}{r}\text { Mean } \pm \text { St. } \\
\text { deviation }\end{array}$ & $\begin{array}{c}2.63 \pm \\
0.29\end{array}$ & $\begin{array}{c}1.00 \pm \\
0.11\end{array}$ & $\begin{array}{c}2.35 \pm \\
0.30\end{array}$ & $\begin{array}{c}0.89 \pm \\
0.11\end{array}$ & $\begin{array}{c}3.98 \pm \\
0.21\end{array}$ & $\begin{array}{c}1.00 \pm \\
0.05\end{array}$ & $\begin{array}{c}3.99 \pm \\
0.35\end{array}$ & $\begin{array}{c}1.00 \pm \\
0.08\end{array}$ \\
\hline Median & 2.63 & 1.00 & 2.34 & 0.89 & 3.99 & 1.00 & 4.99 & 1.00 \\
\hline
\end{tabular}



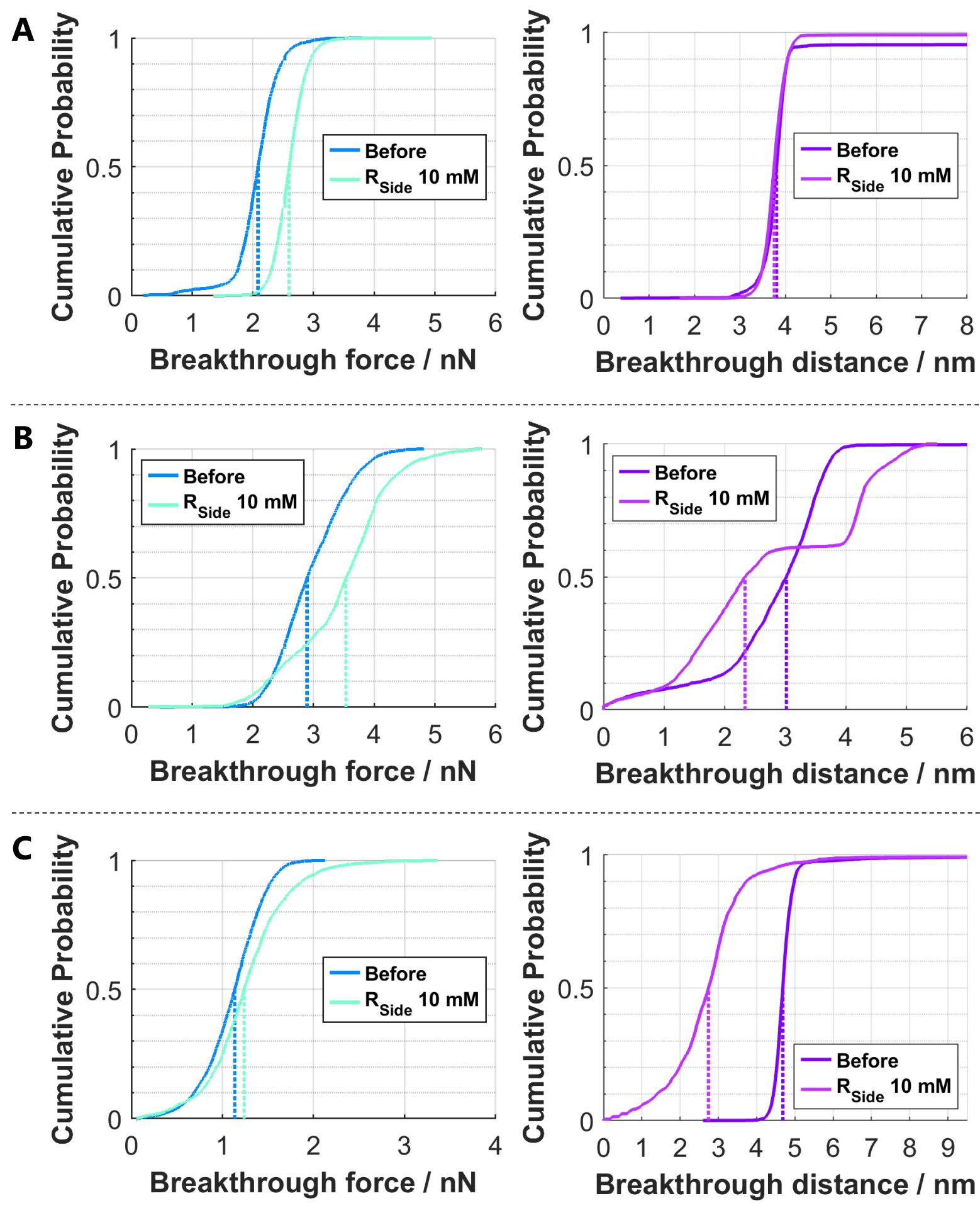

Figure A 2.2: Results of individual breakthrough force spectroscopy experiments on POPC:BP 99:1 membranes with $10 \mathrm{mM} N$-Propylguanidine (Rside). The left plots represent breakthrough force results, the right plots show breakthrough distance results. The part of the curves with the largest slope would be the peak in a histogram. Medians corresponding to this peak are indicated as dashed lines. A: Experiment 1; B: Experiment 2; C: Experiment 3. 
Table A 2.2: Means, medians and standard deviations for all breakthrough force spectroscopy experiments on a POPC:BP 99:1 lipid composition with $10 \mathrm{mM} \mathrm{R}_{\text {side, }}$ as shown in Figure A 2.2.

\section{Breakthrough Force}

\section{Before $\quad \mathbf{R}_{\text {Side }} 10 \mathrm{mM}$}

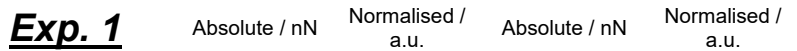

\section{Breakthrough Distance}

Before $\quad R_{\text {Side }} 10 \mathrm{mM}$

\begin{tabular}{r|c|c|c|c|c|c|c|c|c|}
\hline Mean \pm St. & $2.07 \pm$ & $1.00 \pm$ & $2.61 \pm$ & $1.25 \pm$ & $4.88 \pm$ & $1.28 \pm$ & $3.84 \pm$ & $1.01 \pm$ \\
deviation & 0.37 & 0.18 & 0.26 & 0.12 & 5.71 & 1.50 & 0.99 & 0.26 \\
\hline Median & 2.60 & 1.00 & 2.60 & 1.25 & 3.81 & 1.00 & 3.76 & 0.99 \\
\hline
\end{tabular}

Exp. 2

\begin{tabular}{|r|c|c|c|c|c|c|c|c|}
\hline Mean \pm St. & $2.95 \pm$ & $1.02 \pm$ & $3.44 \pm$ & $1.19 \pm$ & $2.79 \pm$ & $0.93 \pm$ & $2.74 \pm$ & $0.91 \pm$ \\
\hline deviation & 0.56 & 0.19 & 0.82 & 0.28 & 1.05 & 0.35 & 1.43 & 0.47 \\
\hline Median & 2.89 & 1.00 & 3.53 & 1.22 & 3.02 & 1.00 & 2.33 & 0.77 \\
\hline
\end{tabular}

Exp. 3

\begin{tabular}{|r|c|c|c|c|c|c|c|c|}
\hline Mean \pm St. & $1.11 \pm$ & $0.98 \pm$ & $1.26 \pm$ & $1.11 \pm$ & $5.10 \pm$ & $1.09 \pm$ & $3.24 \pm$ & $0.69 \pm$ \\
\hline deviation & 0.32 & 0.28 & 0.45 & 0.40 & 6.16 & 1.32 & 10.38 & 2.22 \\
\hline Median & 1.13 & 1.00 & 1.24 & 1.09 & 4.68 & 1.00 & 2.74 & 0.59 \\
\hline
\end{tabular}



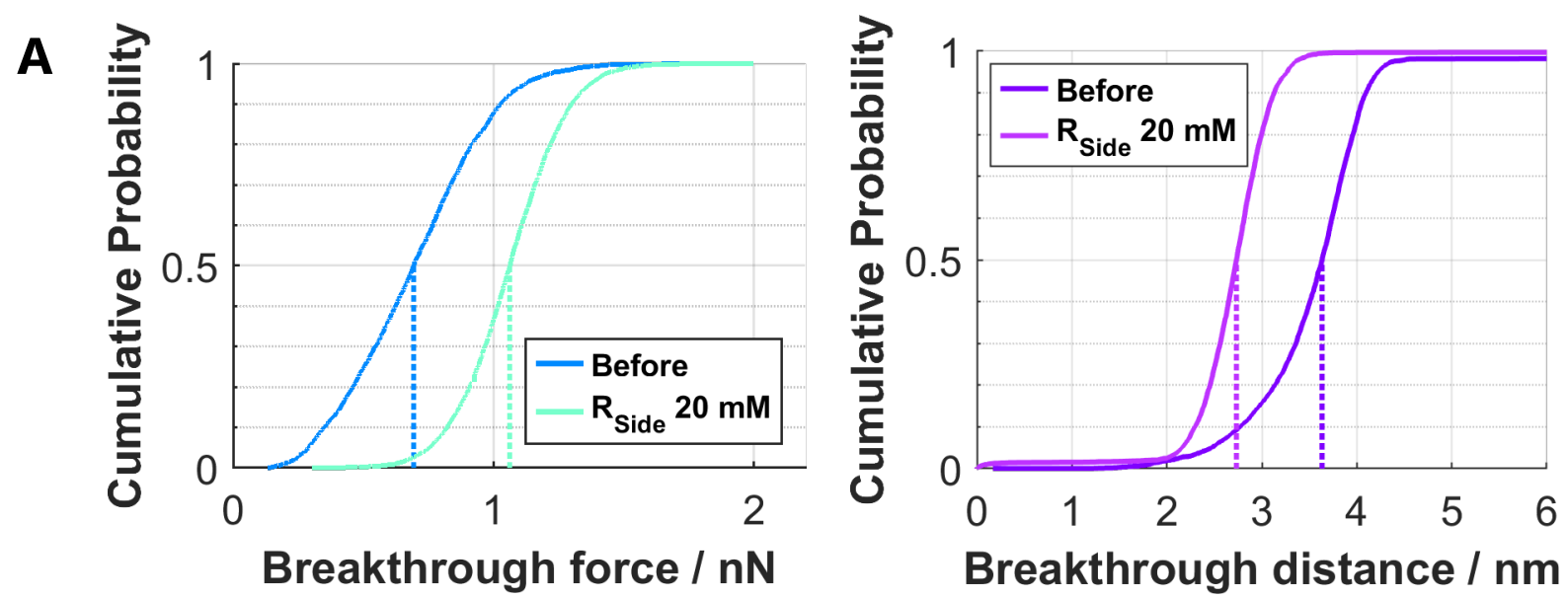

B
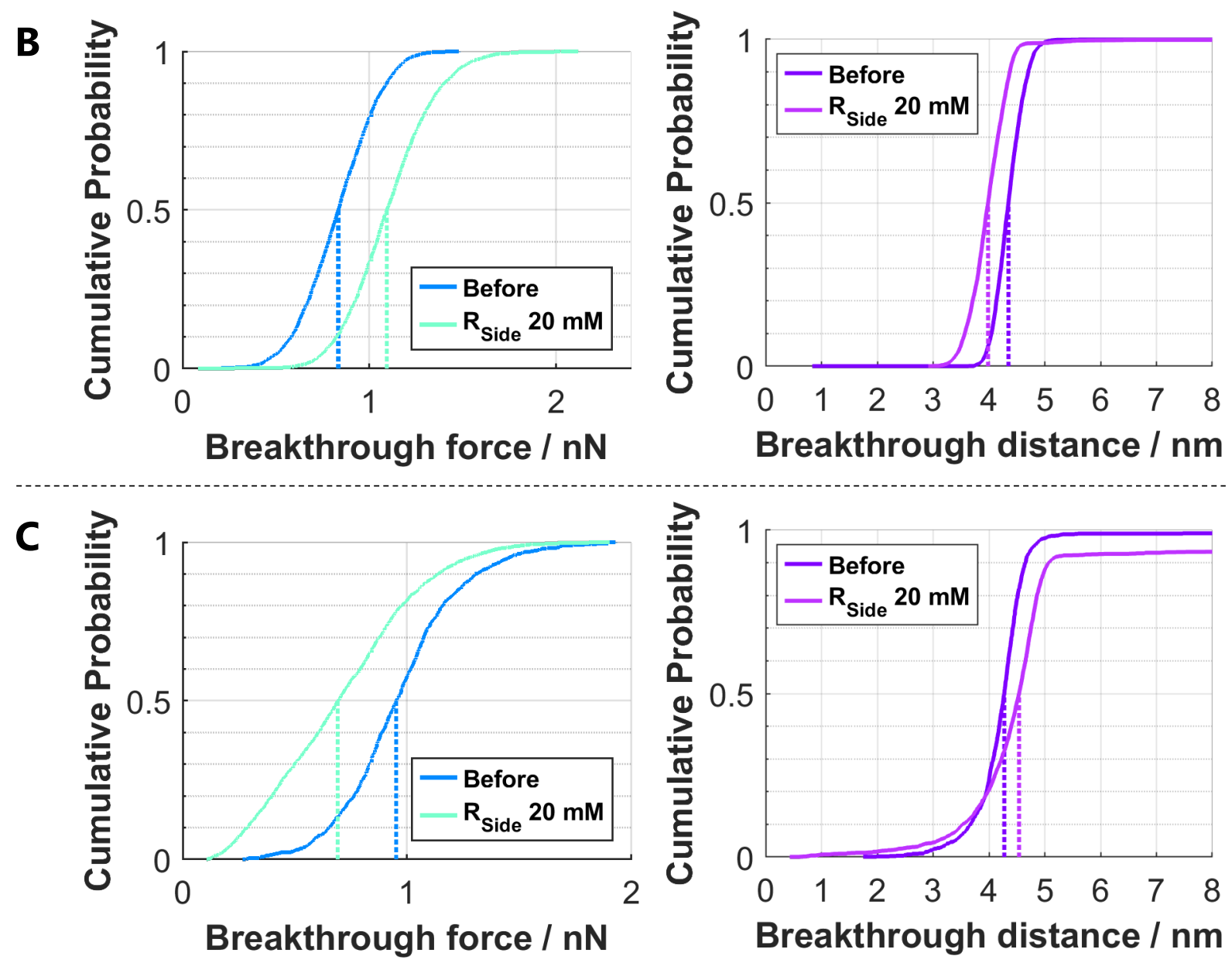

Figure A 2.3: Results of individual breakthrough force spectroscopy experiments on POPC:BP 99:1 membranes with $20 \mathrm{mM} \mathrm{N}$-Propylguanidine $\left(\mathrm{R}_{\text {side }}\right)$. The left plots represent breakthrough force results, the right plots show breakthrough distance results. The part of the curves with the largest slope would be the peak in a histogram. Medians corresponding to this peak are indicated as dashed lines. A: Experiment 1; B: Experiment 2; C: Experiment 3. 
Table A 2.3: Means, medians and standard deviations for all breakthrough force spectroscopy experiments on a POPC:BP 99:1 lipid composition with 20 mM R $R_{\text {side, }}$ as shown in Figure A 2.3.

\section{Breakthrough Force}

\begin{tabular}{|c|c|c|c|c|c|c|c|c|}
\hline \multirow[b]{2}{*}{ Exp. 1} & \multicolumn{2}{|c|}{ Before } & \multicolumn{2}{|c|}{$\mathbf{R}_{\text {Side }} 20 \mathrm{mM}$} & \multicolumn{2}{|c|}{ Before } & \multicolumn{2}{|c|}{$\mathbf{R}_{\text {Side }} 20 \mathrm{mM}$} \\
\hline & Absolute / $\mathrm{nN}$ & $\begin{array}{l}\text { Normalised / } \\
\text { a.u. }\end{array}$ & Absolute / $\mathrm{nN}$ & $\begin{array}{l}\text { Normalised / } \\
\text { a.u. }\end{array}$ & Absolute / nm & $\begin{array}{c}\text { Normalised / } \\
\text { a.u. }\end{array}$ & Absolute / nm & $\begin{array}{l}\text { Normalised / } \\
\text { a.u. }\end{array}$ \\
\hline $\begin{array}{r}\text { Mean } \pm \text { St. } \\
\text { deviation }\end{array}$ & $\begin{array}{c}0.70 \pm \\
0.26\end{array}$ & $\begin{array}{c}1.01 \pm \\
0.37\end{array}$ & $\begin{array}{l}1.06 \pm \\
0.19\end{array}$ & $\begin{array}{c}1.53 \pm \\
0.27\end{array}$ & $\begin{array}{c}3.98 \pm \\
6.23\end{array}$ & $\begin{array}{c}1.10 \pm \\
1.71\end{array}$ & $\begin{array}{c}2.75 \pm \\
1.71\end{array}$ & $\begin{array}{c}0.76 \pm \\
0.42\end{array}$ \\
\hline Median & 0.69 & 1.00 & 1.06 & 1.53 & 3.63 & 1.00 & 2.73 & 0.75 \\
\hline \multicolumn{9}{|l|}{ Exp. 2} \\
\hline $\begin{array}{r}\text { Mean } \pm \text { St } \\
\text { deviation }\end{array}$ & $\begin{array}{c}0.83 \pm \\
0.20\end{array}$ & $\begin{array}{l}1.00 \pm \\
0.24\end{array}$ & $\begin{array}{c}1.10 \pm \\
0.23\end{array}$ & $\begin{array}{l}1.33 \pm \\
0.27\end{array}$ & $\begin{array}{l}4.38 \pm \\
0.75\end{array}$ & $\begin{array}{l}1.01 \pm \\
0.17\end{array}$ & $\begin{array}{c}4.04 \pm \\
1.74\end{array}$ & $\begin{array}{c}0.93 \pm \\
0.40\end{array}$ \\
\hline Median & 0.83 & 1.00 & 1.09 & 1.31 & 4.35 & 1.00 & 3.98 & 0.92 \\
\hline \multicolumn{9}{|l|}{ Exp. 3} \\
\hline $\begin{array}{r}\text { Mean } \pm \text { St. } \\
\text { deviation }\end{array}$ & $\begin{array}{c}0.87 \pm \\
0.30\end{array}$ & $\begin{array}{c}1.00 \pm \\
0.34\end{array}$ & $\begin{array}{c}1.39 \pm \\
0.39\end{array}$ & $\begin{array}{l}1.59 \pm \\
0.45\end{array}$ & $\begin{array}{c}5.01 \pm \\
8.31\end{array}$ & $\begin{array}{c}1.13 \pm \\
1.87\end{array}$ & $\begin{array}{c}4.68 \pm \\
4.57\end{array}$ & $\begin{array}{c}1.05 \pm \\
1.03\end{array}$ \\
\hline Median & 0.87 & 1.00 & 1.39 & 1.59 & 4.45 & 1.00 & 4.34 & 0.98 \\
\hline
\end{tabular}



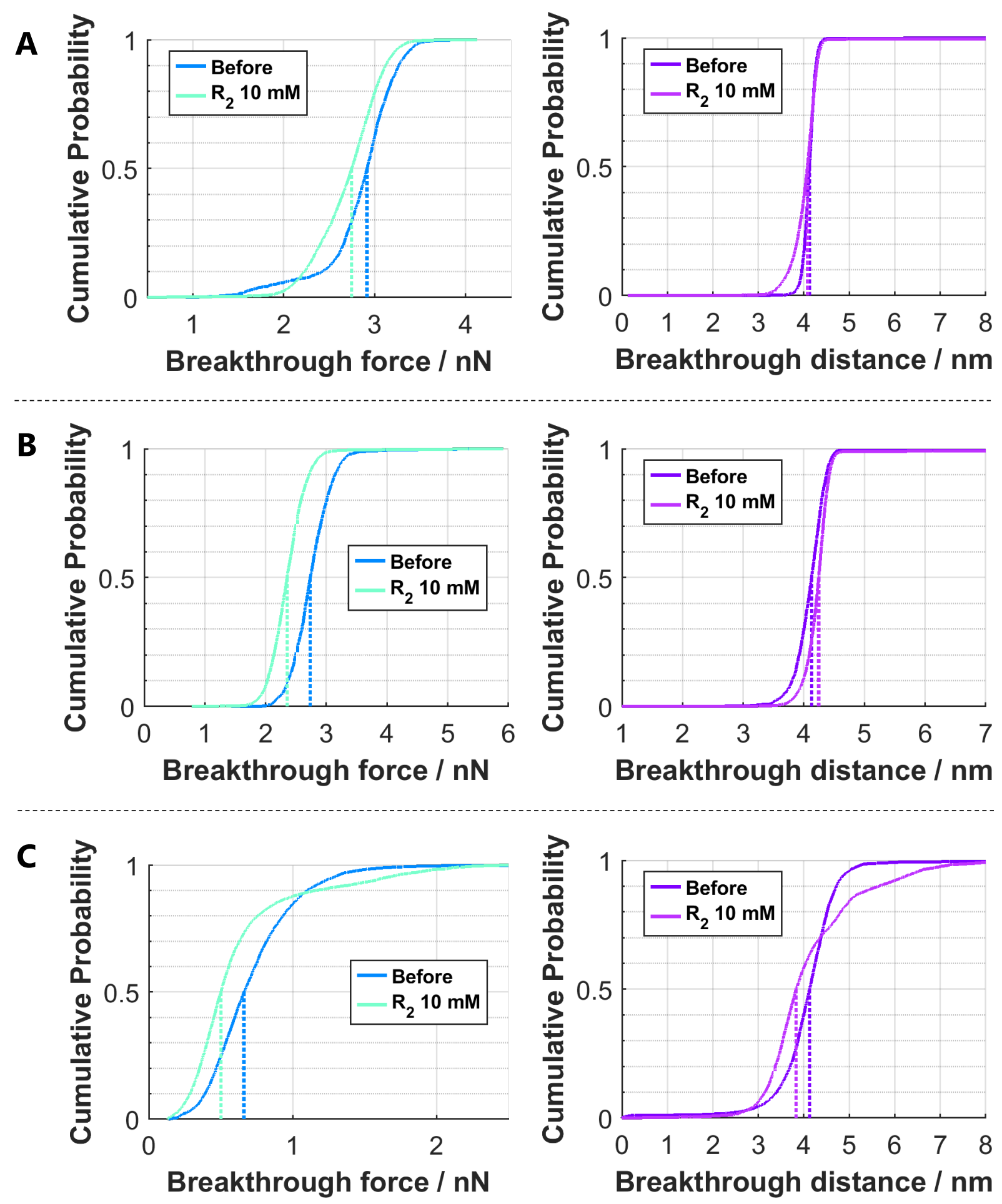

Figure A 2.4: Results of individual breakthrough force spectroscopy experiments on POPC:BP 99:1 membranes with $10 \mathrm{mM}$ of an arginine dipeptide $\left(R_{2}\right)$. The left plots present breakthrough force results, the right plots show breakthrough distance results. The part of the curves with the largest slope would be the peak in a histogram. Medians corresponding to this peak are indicated as dashed lines. A: Experiment 1; B: Experiment 2; $\mathbf{C}$ : Experiment 3. 
Table A 2.4: Means, medians and standard deviations for all breakthrough force spectroscopy experiments on a POPC:BP 99:1 lipid composition with $10 \mathrm{mM} \mathrm{R}$, as shown in Figure $\mathbf{A}$ 2.4.

\section{Breakthrough Force}

Before
$\mathrm{R}_{2} 10 \mathrm{mM}$

\section{Breakthrough Distance}

Before
$R_{2} 10 \mathrm{mM}$

\begin{tabular}{|c|c|c|c|c|c|c|c|c|}
\hline Exp. 1 & Absolute / nN & $\begin{array}{l}\text { Normalised / } \\
\text { a.u. }\end{array}$ & Absolute / nN & $\begin{array}{l}\text { Normalised / } \\
\text { a.u. }\end{array}$ & Absolute / $\mathrm{nm}$ & $\begin{array}{l}\text { Normalised / } \\
\text { a.u. }\end{array}$ & Absolute / nm & $\begin{array}{l}\text { Normalised / } \\
\text { a.u. }\end{array}$ \\
\hline $\begin{array}{r}\text { Mean } \pm \text { St. } \\
\text { deviation }\end{array}$ & $\begin{array}{c}2.85 \pm \\
0.41\end{array}$ & $\begin{array}{c}0.98 \pm \\
0.14\end{array}$ & $\begin{array}{c}2.70 \pm \\
0.36\end{array}$ & $\begin{array}{c}0.93 \pm \\
0.12\end{array}$ & $\begin{array}{c}4.12 \pm \\
0.26\end{array}$ & $\begin{array}{c}1.00 \pm \\
0.06\end{array}$ & $\begin{array}{c}4.07 \pm \\
1.12\end{array}$ & $\begin{array}{c}0.99 \pm \\
0.27\end{array}$ \\
\hline Median & 2.92 & 1.00 & 2.75 & 0.94 & 4.12 & 1.00 & 4.08 & 0.99 \\
\hline \multicolumn{9}{|l|}{ Exp. 2} \\
\hline $\begin{array}{r}\text { Mean } \pm \text { St } \\
\text { deviation }\end{array}$ & $\begin{array}{c}2.76 \pm \\
0.33\end{array}$ & $\begin{array}{c}1.01 \pm \\
0.12\end{array}$ & $\begin{array}{c}2.37 \pm \\
0.31\end{array}$ & $\begin{array}{c}0.87 \pm \\
0.11\end{array}$ & $\begin{array}{c}4.18 \pm \\
1.22\end{array}$ & $\begin{array}{l}1.01 \pm \\
0.30\end{array}$ & $\begin{array}{c}4.39 \pm \\
2.62\end{array}$ & $\begin{array}{c}1.06 \pm \\
0.64\end{array}$ \\
\hline Median & 2.73 & 1.00 & 2.35 & 0.86 & 4.13 & 1.00 & 4.24 & 1.03 \\
\hline \multicolumn{9}{|l|}{ Exp. 3} \\
\hline $\begin{array}{r}\text { Mean } \pm \text { St } \\
\text { deviation }\end{array}$ & $\begin{array}{c}0.72 \pm \\
0.31\end{array}$ & $\begin{array}{l}1.08 \pm \\
0.48\end{array}$ & $\begin{array}{c}0.62 \pm \\
0.41\end{array}$ & $\begin{array}{c}0.94 \pm \\
0.62\end{array}$ & $\begin{array}{l}4.09 \pm \\
0.93\end{array}$ & $\begin{array}{l}0.99 \pm \\
0.23\end{array}$ & $\begin{array}{l}4.20 \pm \\
3.00\end{array}$ & $\begin{array}{l}1.02 \pm \\
0.73\end{array}$ \\
\hline Median & 0.66 & 1.00 & 0.50 & 0.76 & 4.12 & 1.00 & 3.83 & 0.93 \\
\hline
\end{tabular}
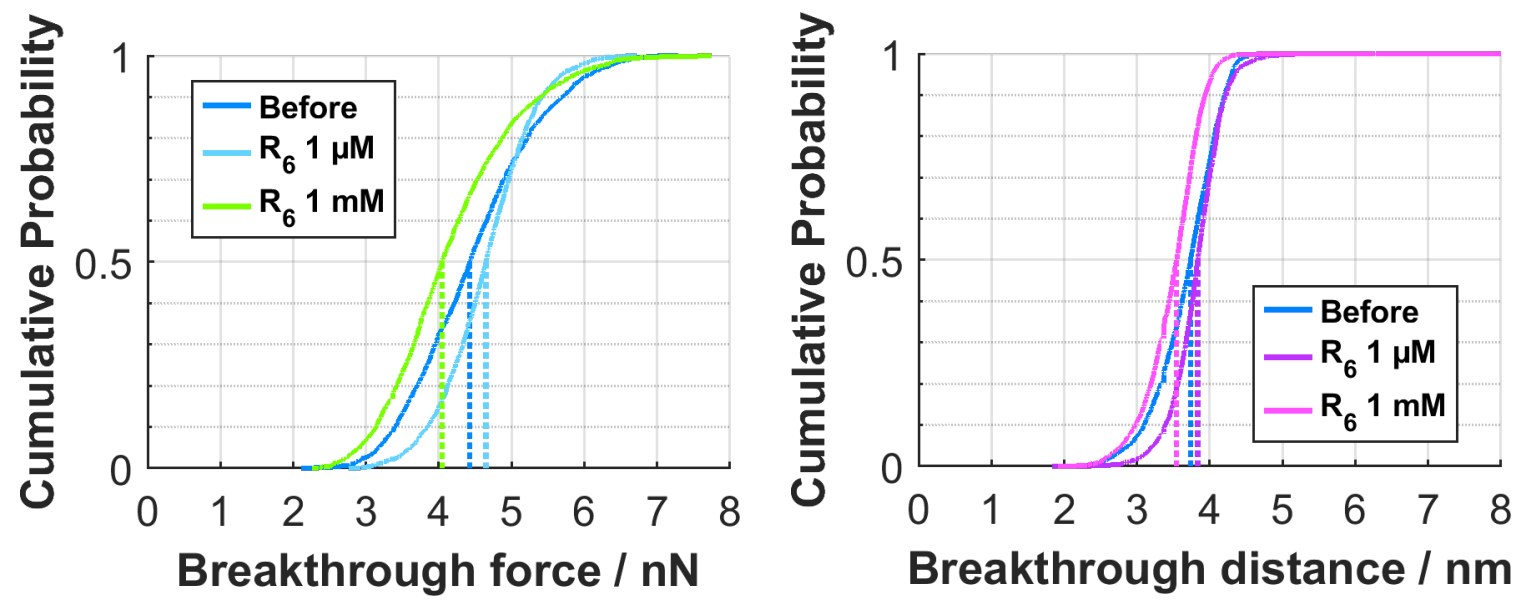

Figure A 2.5: Results of a breakthrough force experiment on a POPC:BP 99:1 bilayer with a hexa-arginine peptide $\left(R_{6}\right)$ in two concentrations. The left plot presents the breakthrough force results, the right plot presents breakthrough distance results.

Table A 2.5: Means, medians and standard deviations of the breakthrough force spectroscopy results presented in Figure A 2.5 .

\section{Breakthrough Force}

\begin{tabular}{|c|c|c|c|c|c|c|}
\hline & \multicolumn{2}{|c|}{ Before } & \multicolumn{2}{|c|}{$R_{6} 1 \mu M$} & \multicolumn{2}{|c|}{$\mathrm{R}_{6} 1 \mathrm{mM}$} \\
\hline & Absolute / $\mathrm{nN}$ & Normalised / a.u. & Absolute / $\mathrm{nN}$ & Normalised / a.u. & Absolute / $\mathrm{nN}$ & Normalised / a.u. \\
\hline an \pm St. deviation & $4.48 \pm 0.87$ & $1.01 \pm 0.20$ & $4.64 \pm 0.62$ & $1.05 \pm 0.14$ & $4.16 \pm 0.88$ & $0.94 \pm 0.20$ \\
\hline Median & 4.42 & 1.00 & 4.65 & 1.05 & 4.04 & 0.91 \\
\hline
\end{tabular}

\section{Breakthrough Distance}

\begin{tabular}{|c|c|c|c|c|c|c|}
\hline & \multicolumn{2}{|c|}{ Before } & \multicolumn{2}{|c|}{$R_{6} 1 \mu M$} & \multicolumn{2}{|c|}{$\mathrm{R}_{6} 1 \mathrm{mM}$} \\
\hline & Absolute / nm & Normalised / a.u. & Absolute / nm & Normalised / a.u. & Absolute / nm & Normalised / a.u. \\
\hline 年 & $3.68 \pm 0.43$ & $0.99 \pm 0.12$ & $3.82 \pm 0.35$ & $1.02 \pm 0.09$ & $3.50 \pm 0.40$ & $0.94 \pm 0.11$ \\
\hline Median & 3.74 & 1.00 & 3.83 & 1.03 & 3.54 & 0.95 \\
\hline
\end{tabular}


3. Results of individual breakthrough force spectroscopy experiments on POPC:POPG:BP 49.5:49.5:1 bilayers
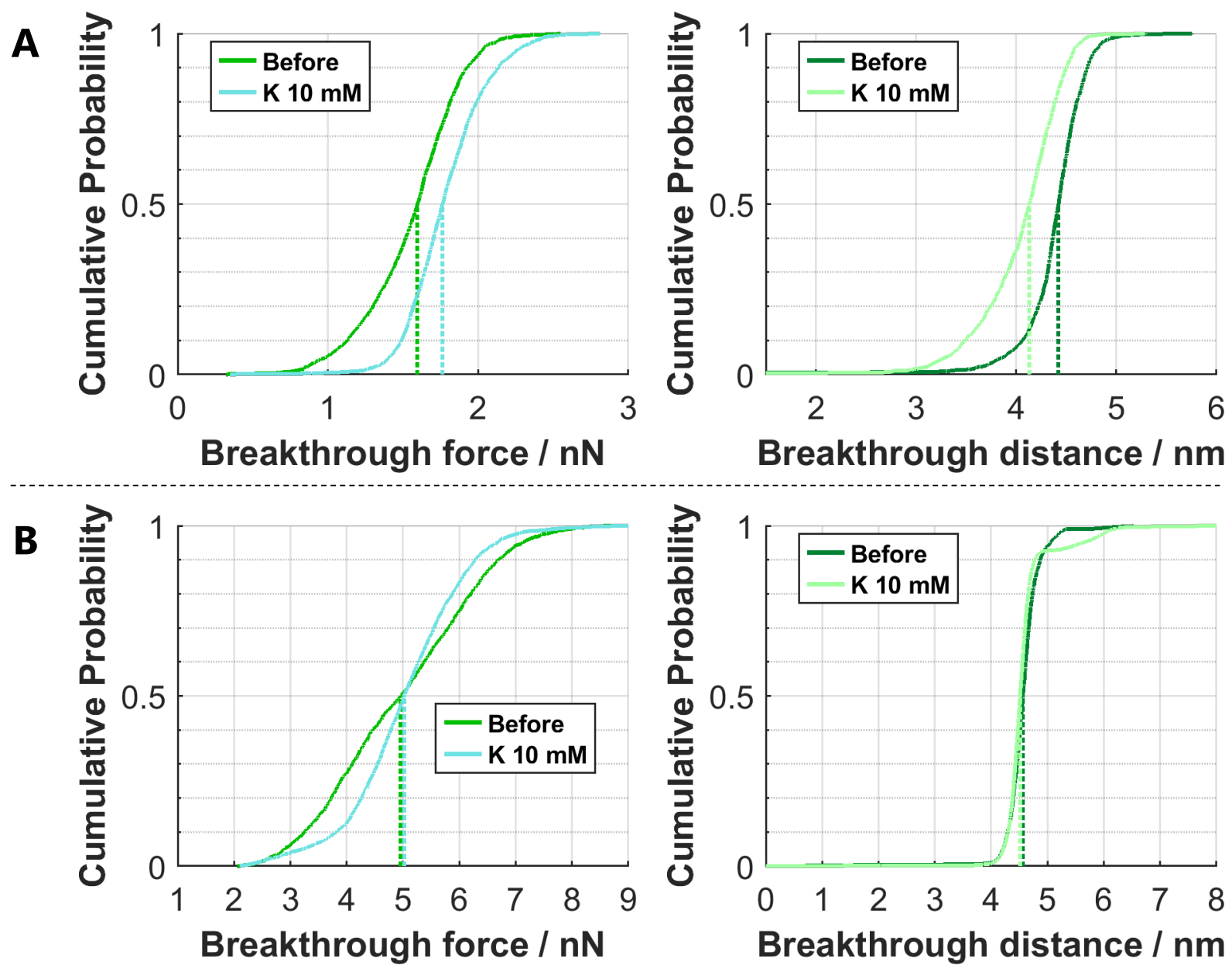

Figure A 3.2.1: Results of individual breakthrough force spectroscopy experiments on POPC:POPG:BP 49.5:49.5:1 membranes with lysine $(K)$. The left plots represent breakthrough force results, the right plots show breakthrough distance results. The part of the curves with the largest slope would be the peak in a histogram. Medians corresponding to this peak are indicated as dashed lines. A: Experiment 1; $\mathbf{B}$ : Experiment 2.

Table A 3.1: Means, medians and standard deviations of datasets presented in the cumulative plots of Figure A 3.2.1.

\section{Breakthrough Force}

\section{Before}

K $10 \mathrm{mM}$

\begin{tabular}{|r|c|c|c|c|}
\hline Exp. 1 & Absolute $/ \mathrm{nN}$ & $\begin{array}{c}\text { Normalised } / \\
\text { a.u. }\end{array}$ & $\begin{array}{c}\text { Absolute } / \mathrm{nN} \\
\text { Normalised } /\end{array}$ \\
\hline a.u. \\
\hline dean \pm St. & $1.56 \pm$ & $0.98 \pm$ & $1.78 \pm$ & $1.12 \pm$ \\
\hline Median & 0.32 & 0.20 & 0.27 & 0.17 \\
\hline
\end{tabular}

\section{Breakthrough Distance}

\section{Before}

\section{$\mathrm{K} 10 \mathrm{mM}$}

\begin{tabular}{|c|c|c|c|}
\hline Absolute / $\mathrm{nm}$ & $\begin{array}{c}\text { Normalised / } \\
\text { a.u. }\end{array}$ & Absolute / $\mathrm{nm}$ & $\begin{array}{c}\text { Normalised / } \\
\text { a.u. }\end{array}$ \\
\hline $4.38 \pm$ & $0.99 \pm$ & $4.05 \pm$ & $0.91 \pm$ \\
\hline 0.41 & 0.09 & 0.45 & 0.10 \\
\hline 4.42 & 1.00 & 4.13 & 0.93 \\
\hline
\end{tabular}

Exp. 2

\begin{tabular}{|r|c|c|c|c|c|c|c|c|}
\hline Mean \pm St. & $4.97 \pm$ & $1.00 \pm$ & $5.03 \pm$ & $1.02 \pm$ & $4.57 \pm$ & $1.00 \pm$ & $4.58 \pm$ & $1.01 \pm$ \\
\hline deviation & 1.32 & 0.27 & 1.03 & 0.21 & 0.35 & 0.08 & 0.43 & 0.10 \\
\hline Median & 4.95 & 1.00 & 5.02 & 1.01 & 4.56 & 1.00 & 4.51 & 0.99 \\
\hline
\end{tabular}



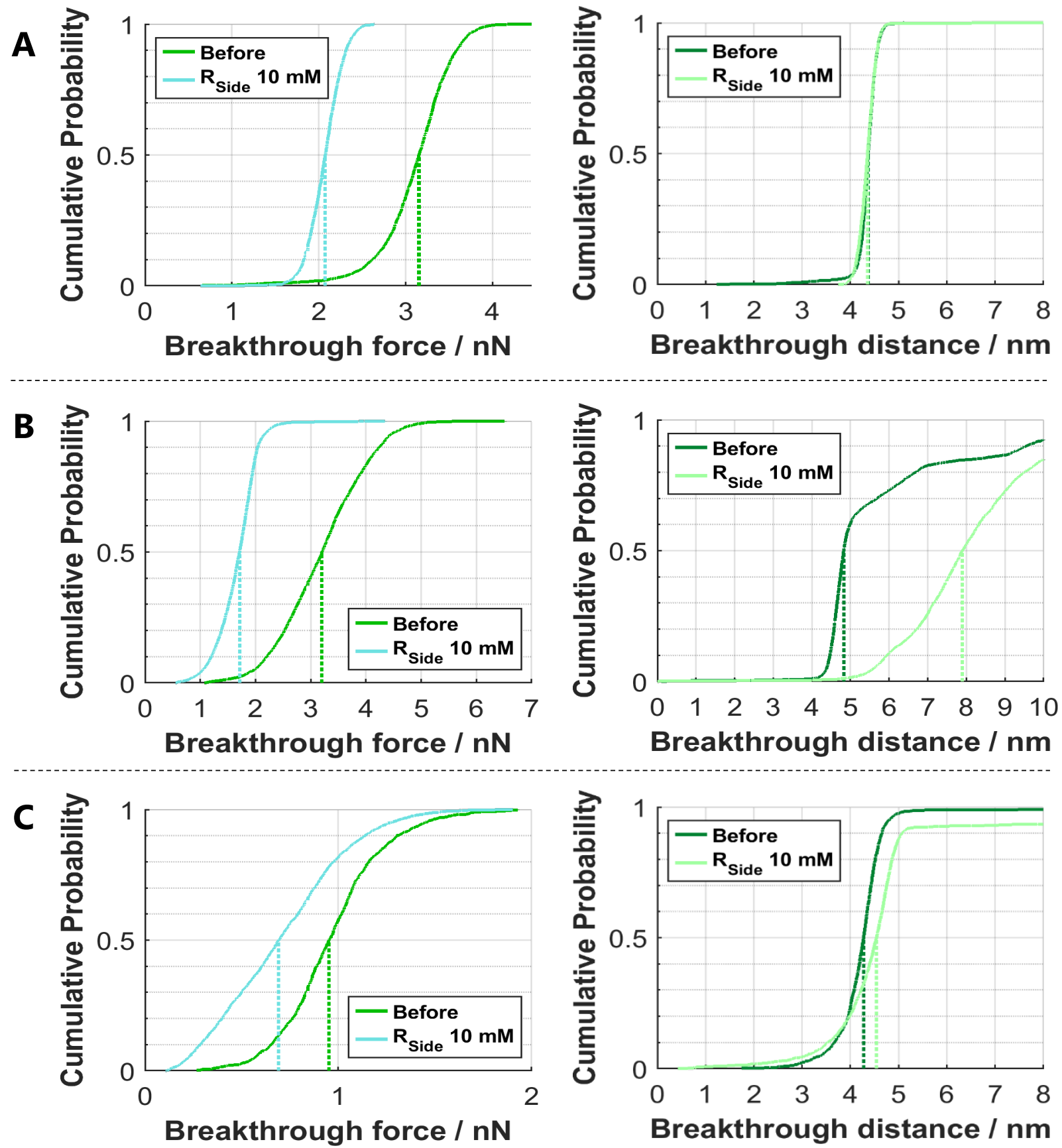

Figure A 3.2: Results of individual breakthrough force spectroscopy experiments on POPC:POPG:BP 49.5:49.5:1 membranes with $10 \mathrm{mM} \mathrm{N}$-Propylguanidine ( $R_{\text {side). }}$. The left plots represent breakthrough force results, the right plots show breakthrough distance results. The part of the curves with the largest slope would be the peak in a histogram. Medians corresponding to this peak are indicated as dashed lines. A: Experiment 1; B: Experiment 2; C: Experiment 3. 
Table A 3.2: Means, medians and standard deviations of datasets presented in the cumulative plots of Figure A 3.2.

\section{Breakthrough Force}

\section{Before}

Exp. 1
Mean \pm
deviation
Med
Exp. 2
Mean \pm

Mean \pm St

deviation

Median 3.20

Exp. 3

Mean \pm S

deviation $\quad 0.27$

Median 0.95

$0.24 \quad 0.36$

1.71

$0.71 \pm$

$\mathbf{R}_{\text {Side }} 10 \mathrm{mM}$

\section{Breakthrough Distance}

\section{Before $\quad R_{\text {Side }} 10 \mathrm{mM}$}

\begin{tabular}{|c|c|c|c|c|c|c|}
\hline $\begin{array}{c}\text { Normalised / } \\
\text { a.u. }\end{array}$ & Absolute / $\mathrm{nN}$ & $\begin{array}{c}\text { Normalised / } \\
\text { a.u. }\end{array}$ & $\begin{array}{c}\text { Absolute / } \mathrm{nm} \\
0.99 \pm\end{array}$ & $\begin{array}{c}\text { Normalised / } \\
\text { a.u. }\end{array}$ & $\begin{array}{c}\text { Absolute / } \mathrm{nm} \\
0.07 \pm\end{array}$ & $\begin{array}{c}\text { Normalised / } \\
\text { a.u. }\end{array}$ \\
\hline 0.14 & $0.66 \pm$ & $4.35 \pm$ & $1.00 \pm$ & $4.36 \pm$ & $1.00 \pm$ \\
\hline 1.00 & 2.07 & 0.06 & 0.26 & 0.06 & 0.23 & 0.05 \\
\hline
\end{tabular}

\begin{tabular}{|l|l|l|}
\hline $1.00 \pm$ & $1.67 \pm$ & $0.52 \pm$ \\
\hline
\end{tabular}

\begin{tabular}{l|l|l}
\hline 0.24 & 0.36 & 0.11 \\
\hline
\end{tabular}

$5.83 \pm$

0.11

4.82

$1.21 \pm$

$8.62 \pm$

$1.79 \pm$

0.53

$0.74 \pm$

0.34

$4.58 \pm$

0.52

10.09

2.09

1.64

\begin{tabular}{r|c|c|c|c|c|c|c|c|}
\hline Mean \pm St. & $0.97 \pm$ & $1.02 \pm$ & $0.71 \pm$ & $0.74 \pm$ & $4.58 \pm$ & $1.07 \pm$ & $6.07 \pm$ & $1.42 \pm$ \\
\hline deviation & 0.27 & 0.28 & 0.32 & 0.34 & 5.68 & 1.33 & 13.33 & 3.12 \\
\hline Median & 0.95 & 1.00 & 0.69 & 0.73 & 4.27 & 1.00 & 4.54 & 1.06 \\
\hline
\end{tabular}



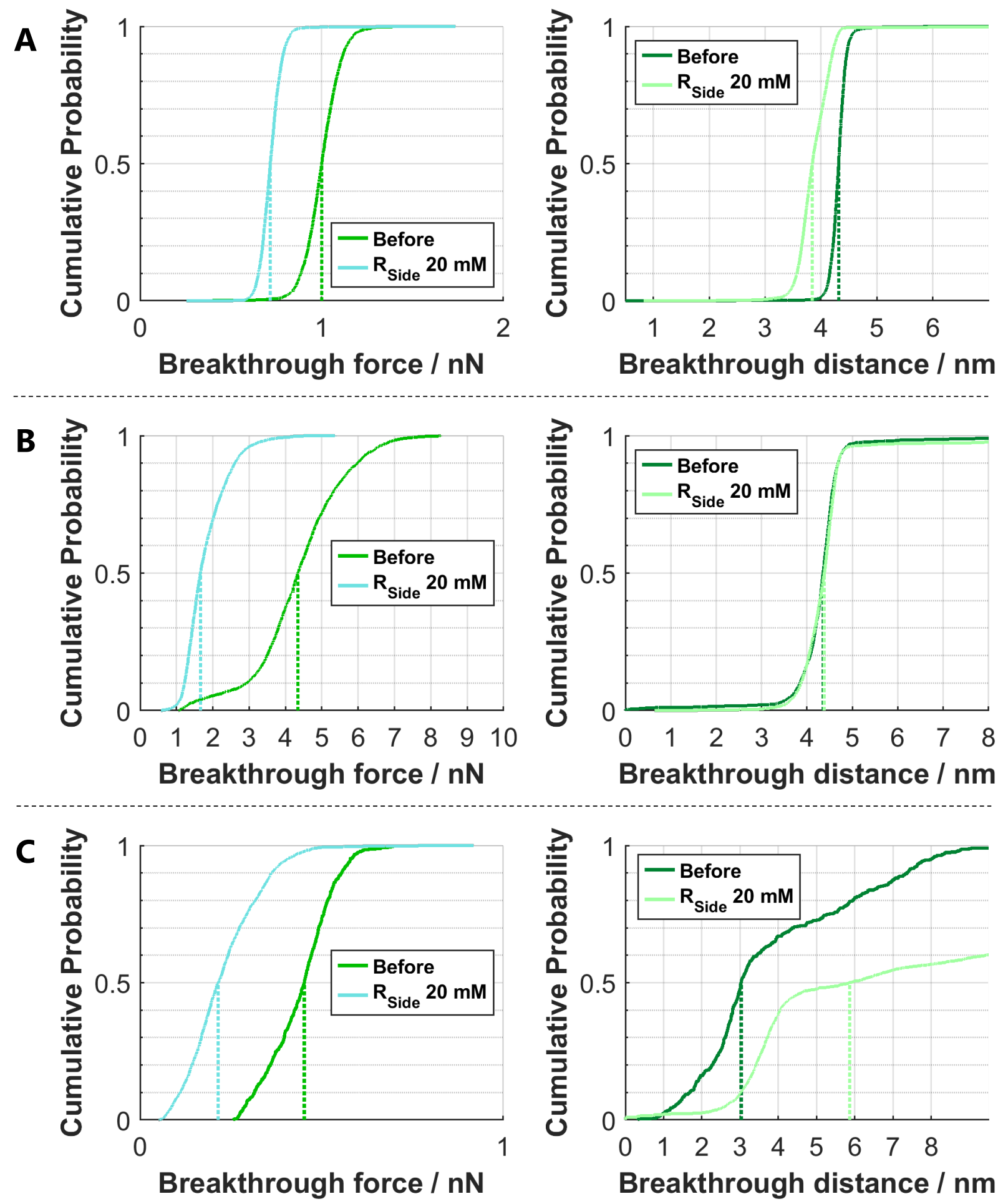

Figure A 3.3: Results of individual breakthrough force spectroscopy experiments on POPC:POPG:BP 49.5:49.5:1 membranes with $20 \mathrm{mM} \mathrm{N}$-Propylguanidine ( $\mathrm{R}_{\text {side) }}$. The left plots represent breakthrough force results, the right plots show breakthrough distance results. The part of the curves with the largest slope would be the peak in a histogram. Medians corresponding to this peak are indicated as dashed lines. A: Experiment 1; B: Experiment 2; C: Experiment 3. 
Table A 3.3: Means, medians and standard deviations of datasets presented in the cumulative plots of Figure A 3.3.

\section{Breakthrough Force}

Before
$\mathbf{R}_{\text {Side }} 20 \mathrm{mM}$

\begin{tabular}{|c|c|c|c|c|c|c|c|c|}
\hline Exp. 1 & Absolute / nN & $\begin{array}{l}\text { Normalised / } \\
\text { a.u. }\end{array}$ & Absolute / $\mathrm{nN}$ & $\begin{array}{l}\text { Normalised / } \\
\text { a.u. }\end{array}$ & Absolute / nm & $\begin{array}{l}\text { Normalised / } \\
\text { a.u. }\end{array}$ & Absolute / nm & $\begin{array}{l}\text { Normalised / } \\
\text { a.u. }\end{array}$ \\
\hline $\begin{array}{r}\text { Mean } \pm \text { St. } \\
\text { deviation }\end{array}$ & $\begin{array}{c}2.29 \pm \\
0.22\end{array}$ & $\begin{array}{c}1.00 \pm \\
0.10\end{array}$ & $\begin{array}{c}1.65 \pm \\
0.14\end{array}$ & $\begin{array}{c}0.72 \pm \\
0.06\end{array}$ & $\begin{array}{c}4.31 \pm \\
0.25\end{array}$ & $\begin{array}{c}1.00 \pm \\
0.06\end{array}$ & $\begin{array}{c}3.89 \pm \\
0.85\end{array}$ & $\begin{array}{c}0.90 \pm \\
0.20\end{array}$ \\
\hline Median & 2.29 & 1.00 & 1.64 & 0.72 & 4.32 & 1.00 & 3.84 & 0.89 \\
\hline
\end{tabular}

Exp. 2

\begin{tabular}{|r|c|c|c|c|c|c|c|c|}
\hline Mean \pm St. & $4.36 \pm$ & $1.00 \pm$ & $1.81 \pm$ & $0.42 \pm$ & $4.34 \pm$ & $1.00 \pm$ & $4.82 \pm$ & $1.11 \pm$ \\
\hline deviation & 1.25 & 0.29 & 0.59 & 0.14 & 0.97 & 0.22 & 8.38 & 1.93 \\
\hline Median & 4.34 & 1.00 & 1.66 & 0.38 & 4.35 & 1.00 & 4.38 & 1.01 \\
\hline
\end{tabular}

Exp. 3

\begin{tabular}{|r|c|c|c|c|c|c|c|c|}
\hline Mean \pm St. & $0.44 \pm$ & $0.98 \pm$ & $0.23 \pm$ & $0.51 \pm$ & $3.84 \pm$ & $1.27 \pm$ & $9.06 \pm$ & $2.99 \pm$ \\
\hline deviation & 045 & 1.00 & 0.10 & 0.22 & 2.11 & 0.70 & 13.93 & 4.60 \\
\hline Median & 0.45 & 1.00 & 0.22 & 0.48 & 3.03 & 1.00 & 5.86 & 1.94 \\
\hline
\end{tabular}

\section{Before $\quad R_{\text {Side }} 20 \mathrm{mM}$}

20

\section{Breakthrough Distance}



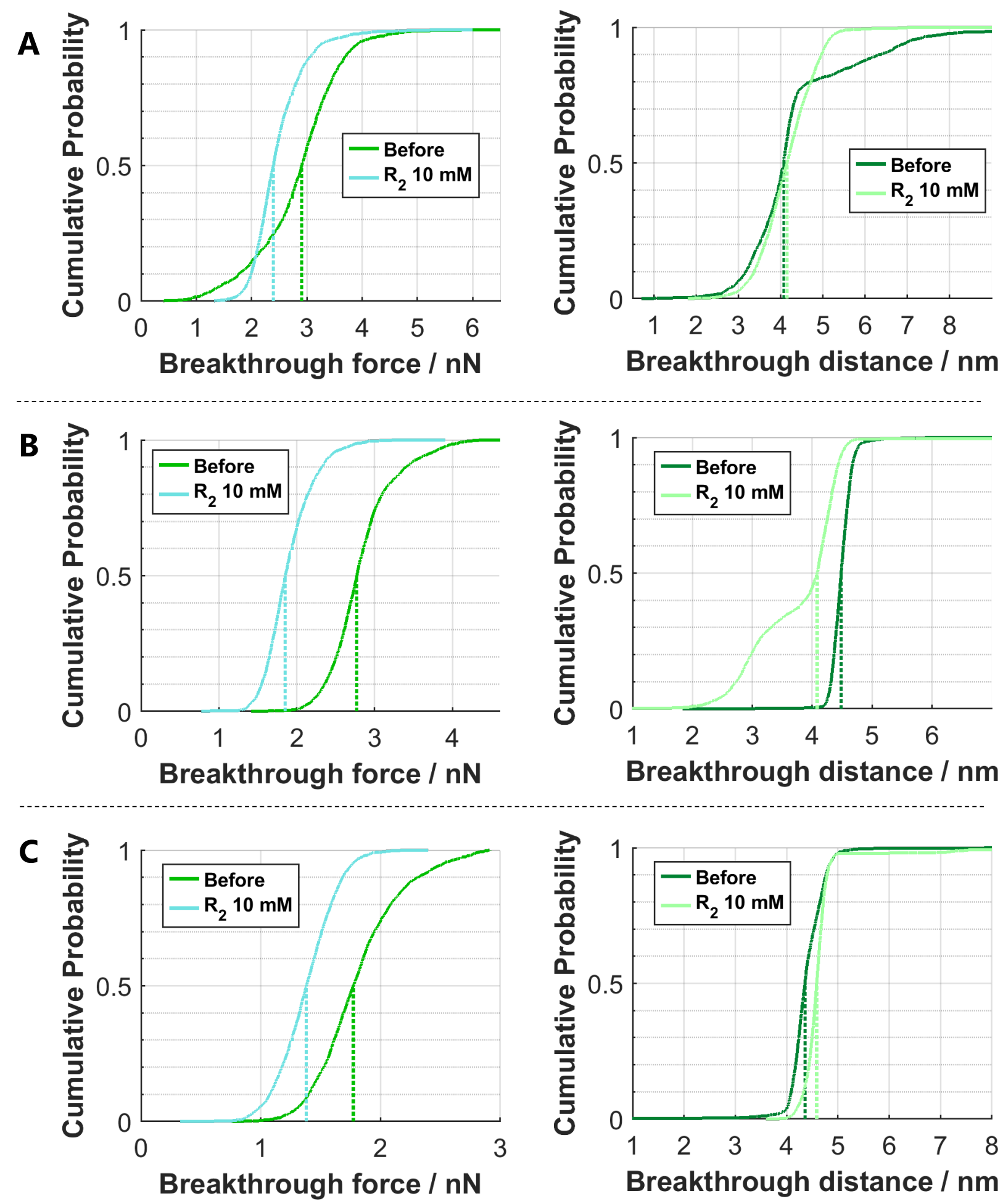

Figure A 3.4: Results of individual breakthrough force spectroscopy experiments on POPC:POPG:BP 49.5:49.5:1 membranes with $10 \mathrm{mM}$ of an arginine dipeptide $\left(\mathrm{R}_{2}\right)$. The left plots represent breakthrough force results, the right plots show breakthrough distance results. The part of the curves with the largest slope would be the peak in a histogram. Medians corresponding to this peak are indicated as dashed lines. A: Experiment 1; B: Experiment 2; C: Experiment 3. 
Table A 3.4: Means, medians and standard deviations of datasets presented in the cumulative plots of Figure A 3.4.

\section{Breakthrough Force}

\section{Before}

$\mathrm{R}_{2} 10 \mathrm{mM}$

\section{Breakthrough Distance}

Before $\quad R_{2} 10 \mathrm{mM}$

\begin{tabular}{|c|c|c|c|c|c|c|c|c|}
\hline Exp. 1 & Absolute / $\mathrm{nN}$ & $\begin{array}{l}\text { Normalised / } \\
\text { a.u. }\end{array}$ & Absolute / $\mathrm{nN}$ & $\begin{array}{l}\text { Normalised / } \\
\text { a.u. }\end{array}$ & Absolute / nm & $\begin{array}{c}\text { Normalised / } \\
\text { a.u. }\end{array}$ & Absolute / nm & $\begin{array}{l}\text { Normalised / } \\
\text { a.u. }\end{array}$ \\
\hline $\begin{array}{r}\text { Mean } \pm \text { St. } \\
\text { deviation }\end{array}$ & $\begin{array}{c}2.83 \pm \\
0.77\end{array}$ & $\begin{array}{c}0.98 \pm \\
0.26\end{array}$ & $\begin{array}{c}2.47 \pm \\
0.48\end{array}$ & $\begin{array}{c}0.85 \pm \\
0.16\end{array}$ & $\begin{array}{c}4.36 \pm \\
1.45\end{array}$ & $\begin{array}{c}1.07 \pm \\
0.36\end{array}$ & $\begin{array}{c}4.16 \pm \\
0.64\end{array}$ & $\begin{array}{c}1.02 \pm \\
0.16\end{array}$ \\
\hline Median & 2.90 & 1.00 & 2.39 & 0.82 & 4.07 & 1.00 & 4.15 & 1.02 \\
\hline
\end{tabular}

Exp. 2

\begin{tabular}{|r|c|c|c|c|c|c|c|c|}
\hline Mean \pm St. & $2.83 \pm$ & $1.02 \pm$ & $1.90 \pm$ & $0.69 \pm$ & $4.49 \pm$ & $1.00 \pm$ & $3.82 \pm$ & $0.85 \pm$ \\
\hline deviation & 0.42 & 0.15 & 0.31 & 0.11 & 0.21 & 0.05 & 1.63 & 0.36 \\
\hline Median & 2.77 & 1.00 & 1.85 & 0.67 & 4.48 & 1.00 & 4.08 & 0.91 \\
\hline
\end{tabular}

Exp. 3

\begin{tabular}{|r|c|c|c|c|c|c|c|c|}
\hline Mean \pm St. & $1.82 \pm$ & $1.02 \pm$ & $1.38 \pm$ & $0.78 \pm$ & $4.39 \pm$ & $1.01 \pm$ & $4.63 \pm$ & $1.06 \pm$ \\
\hline deviation & 0.36 & 0.20 & 0.24 & 0.14 & 0.36 & 0.08 & 0.58 & 0.13 \\
\hline Median & 1.77 & 1.00 & 1.38 & 0.78 & 4.36 & 1.00 & 4.59 & 1.05 \\
\hline
\end{tabular}



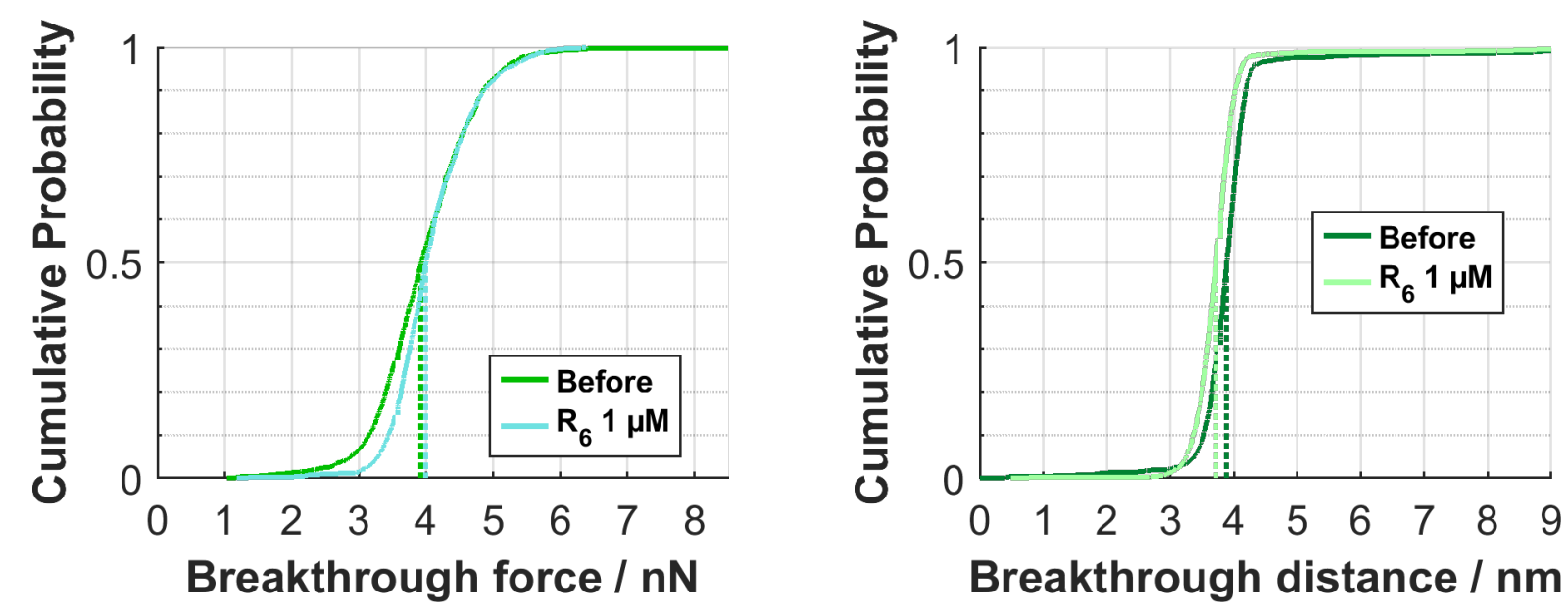

Figure A 3.5: Results of a breakthrough force spectroscopy experiment on POPC:POPG:BP 49.5:49.5:1 membrane, with $1 \mu \mathrm{M}$ of a hexa-arginine peptide $\left(R_{6}\right)$. The left plot presents the breakthrough force results, the right plot shows the breakthrough distance results.

Table A 3.5: Means, medians and standard deviations to the datasets presented in the cumulative plots of Figure A 3.5 .

\section{Breakthrough Force}

\begin{tabular}{|c|c|c|c|c|c|c|c|c|}
\hline & \multicolumn{2}{|c|}{ Before } & \multicolumn{2}{|c|}{$R_{6} 1 \mu M$} & \multicolumn{2}{|c|}{ Before } & \multicolumn{2}{|c|}{$R_{6} 1 \mu M$} \\
\hline & Absolute / $\mathrm{nN}$ & $\begin{array}{c}\text { Normalised / } \\
\text { a.u. }\end{array}$ & Absolute / $\mathrm{nN}$ & $\begin{array}{l}\text { Normalised / } \\
\text { a.u. }\end{array}$ & Absolute / nm & $\begin{array}{c}\text { Normalised / } \\
\text { a.u. }\end{array}$ & Absolute / nm & $\begin{array}{c}\text { Normalised / } \\
\text { a.u. }\end{array}$ \\
\hline $\begin{array}{r}\text { Mean } \pm \text { St } \\
\text { deviation }\end{array}$ & $\begin{array}{c}3.97 \pm \\
0.76\end{array}$ & $\begin{array}{c}1.01 \pm \\
0.19\end{array}$ & $\begin{array}{c}4.08 \pm \\
0.60\end{array}$ & $\begin{array}{c}1.04 \pm \\
0.15\end{array}$ & $\begin{array}{c}4.00 \pm \\
2.55\end{array}$ & $\begin{array}{c}1.03 \pm \\
0.66\end{array}$ & $\begin{array}{c}3.76 \pm \\
0.68\end{array}$ & $\begin{array}{c}0.97 \pm \\
0.17\end{array}$ \\
\hline Median & 3.92 & 1.00 & 4.00 & 1.02 & 3.88 & 1.00 & 3.72 & 0.96 \\
\hline
\end{tabular}



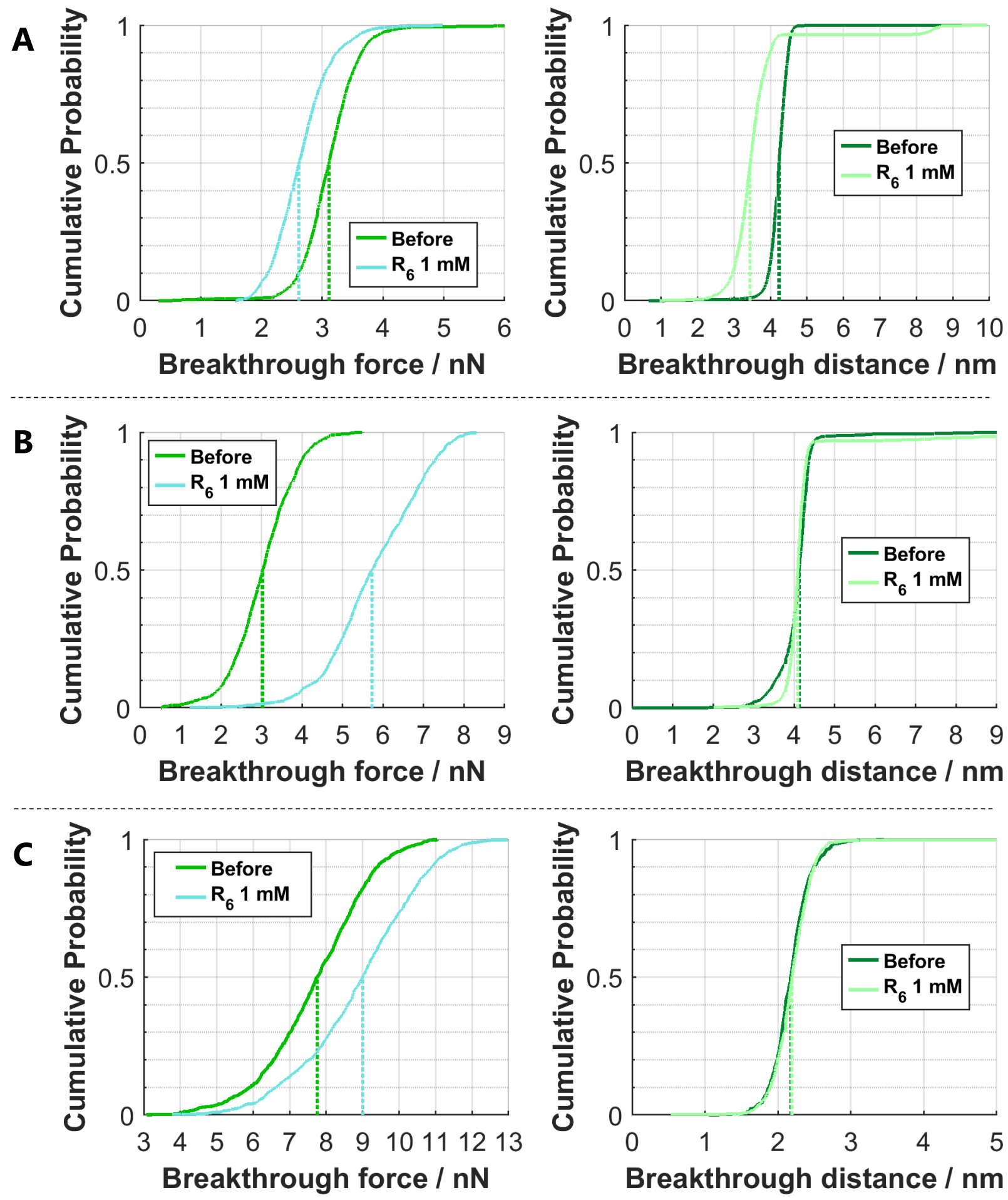

Figure A 3.6: Results of individual breakthrough force spectroscopy experiments on POPC:POPG:BP 49.5:49.5:1 membranes with $1 \mathrm{mM}$ of a hexa-arginine peptide $\left(R_{6}\right)$. The left plots represent breakthrough force results, the right plots show breakthrough distance results. The part of the curves with the largest slope would be the peak in a histogram. Medians corresponding to this peak are indicated as dashed lines. A: Experiment 1; B: Experiment 2; C: Experiment 3. 
Table A 3.6: Means, medians and standard deviations to the datasets presented in the cumulative plots of Figure A 3.6.

\section{Breakthrough Force}

Before $\quad R_{6} 1 \mathrm{mM}$

\section{Breakthrough Distance}

Before $\quad R_{6} 1 \mathrm{mM}$

\begin{tabular}{|c|c|c|c|c|c|c|c|c|}
\hline Exp. 1 & Absolute / $\mathrm{nN}$ & $\begin{array}{c}\text { Normalised / } \\
\text { a.u. }\end{array}$ & Absolute / $\mathrm{nN}$ & $\begin{array}{c}\text { Normalised / } \\
\text { a.u. }\end{array}$ & Absolute / nm & $\begin{array}{c}\text { Normalised / } \\
\text { a.u. }\end{array}$ & Absolute / nm & $\begin{array}{c}\text { Normalised / } \\
\text { a.u. }\end{array}$ \\
\hline $\begin{array}{r}\text { Mean } \pm \text { St } \\
\text { deviation }\end{array}$ & $\begin{array}{c}3.12 \pm \\
0.49\end{array}$ & $\begin{array}{c}1.00 \pm \\
0.16\end{array}$ & $\begin{array}{c}2.65 \pm \\
0.46\end{array}$ & $\begin{array}{c}0.85 \pm \\
0.15\end{array}$ & $\begin{array}{c}4.21 \pm \\
0.31\end{array}$ & $\begin{array}{c}1.00 \pm \\
0.07\end{array}$ & $\begin{array}{c}3.58 \pm \\
0.99\end{array}$ & $\begin{array}{c}0.85 \pm \\
0.23\end{array}$ \\
\hline Median & 3.11 & 1.00 & 2.62 & 0.84 & 4.23 & 1.00 & 3.44 & 0.81 \\
\hline \multicolumn{9}{|l|}{ Exp. 2} \\
\hline $\begin{array}{r}\text { Mean } \pm \text { St } \\
\text { deviation }\end{array}$ & $\begin{array}{c}3.03 \pm \\
0.77\end{array}$ & $\begin{array}{c}1.00 \pm \\
0.26\end{array}$ & $\begin{array}{c}5.77 \pm \\
1.15\end{array}$ & $\begin{array}{c}1.91 \pm \\
0.38\end{array}$ & $\begin{array}{c}4.07 \pm \\
0.63\end{array}$ & $\begin{array}{c}0.99 \pm \\
0.13\end{array}$ & $\begin{array}{c}4.30 \pm \\
1.70\end{array}$ & $\begin{array}{c}1.04 \pm \\
0.41\end{array}$ \\
\hline Median & 3.02 & 1.00 & 5.73 & 1.89 & 4.12 & 1.00 & 4.09 & 0.99 \\
\hline \multicolumn{9}{|l|}{ Exp. 3} \\
\hline $\begin{array}{r}\text { Mean } \pm \text { St. } \\
\text { deviation }\end{array}$ & $\begin{array}{c}7.70 \pm \\
1.40\end{array}$ & $\begin{array}{c}0.99 \pm \\
0.18\end{array}$ & $\begin{array}{c}8.89 \pm \\
1.60\end{array}$ & $\begin{array}{c}1.15 \pm \\
0.21\end{array}$ & $\begin{array}{c}2.18 \pm \\
0.26\end{array}$ & $\begin{array}{c}1.00 \pm \\
0.12\end{array}$ & $\begin{array}{c}2.20 \pm \\
0.32\end{array}$ & $\begin{array}{l}1.01 \pm \\
0.15\end{array}$ \\
\hline Median & 7.75 & 1.00 & 9.00 & 1.16 & 2.18 & 1.00 & 2.19 & 1.01 \\
\hline
\end{tabular}


4. Results of individual breakthrough force spectroscopy experiments on POPC:POPE:BP 49.5:49.5:1 bilayers
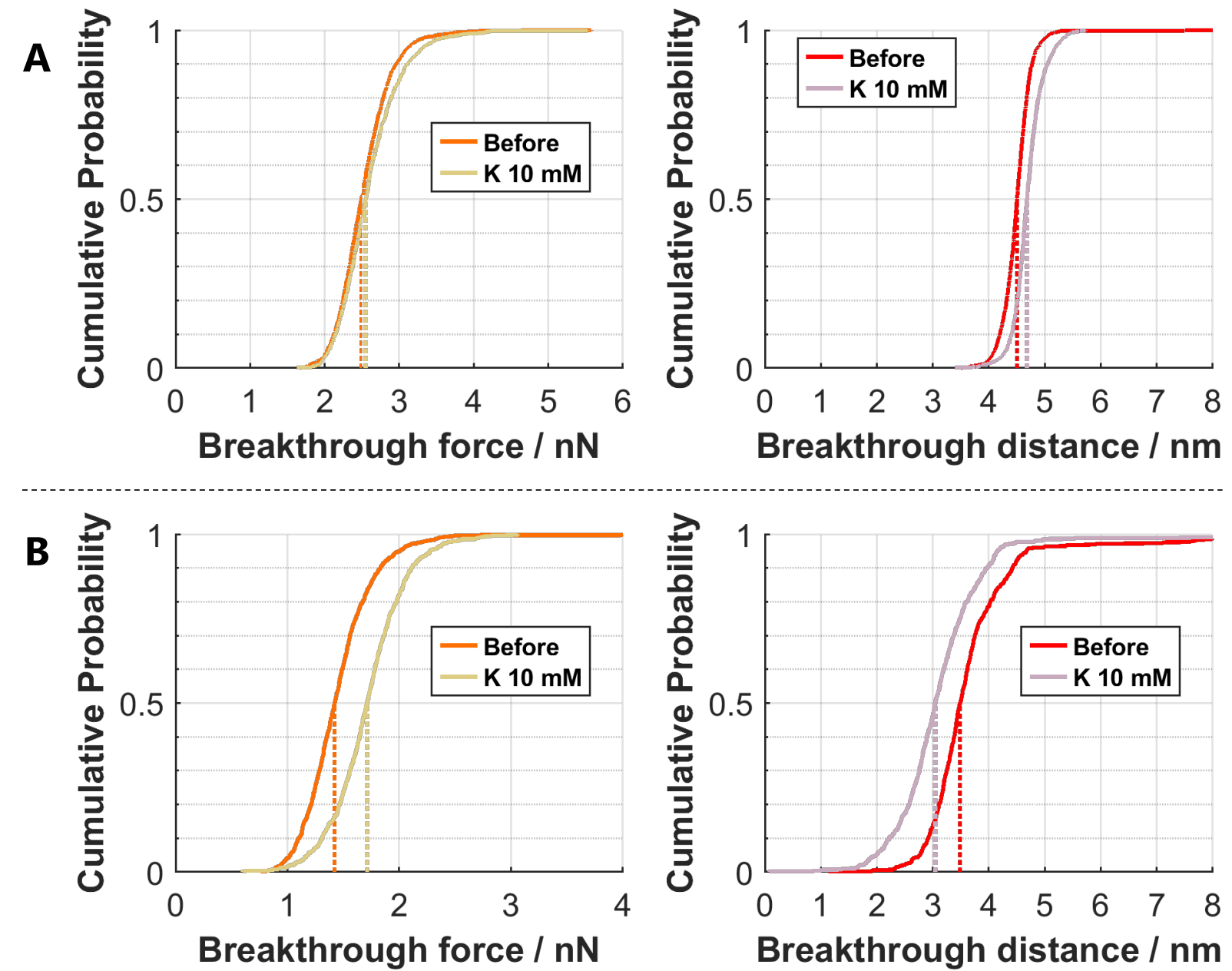

Figure A 4.3.1: Results of individual breakthrough force spectroscopy experiments on POPC:POPE:BP 49.5:49.5:1 membranes with lysine $(K)$. The left plots represent breakthrough force results, the right plots show breakthrough distance results. The part of the curves with the largest slope would be the peak in a histogram. Medians corresponding to this peak are indicated as dashed lines. A: Experiment 1; B: Experiment 2.

Table A 4.1: Means, medians and standard deviations to the datasets presented in the cumulative plots of Figure A 4.3.1.

\section{Breakthrough Force \\ Before \\ K $10 \mathrm{mM}$}

\section{Breakthrough Distance}

Before

K 10 mM

\begin{tabular}{|c|c|c|c|c|c|c|c|c|}
\hline Exp. 1 & Absolute / $\mathrm{nN}$ & $\begin{array}{l}\text { Normalised / } \\
\text { a.u. }\end{array}$ & Absolute / nN & $\begin{array}{l}\text { Normalised / } \\
\text { a.u. }\end{array}$ & Absolute / nm & $\begin{array}{l}\text { Normalised/ } \\
\text { a.u. }\end{array}$ & Absolute / $\mathrm{nm}$ & $\begin{array}{l}\text { Normalised / } \\
\text { a.u. }\end{array}$ \\
\hline $\begin{array}{r}\text { Mean } \pm \text { St. } \\
\text { deviation }\end{array}$ & $\begin{array}{c}2.53 \pm \\
0.36\end{array}$ & $\begin{array}{l}1.01 \pm \\
0.14\end{array}$ & $\begin{array}{c}2.60 \pm \\
0.41\end{array}$ & $\begin{array}{l}1.04 \pm \\
0.16\end{array}$ & $\begin{array}{l}4.50 \pm \\
0.26\end{array}$ & $\begin{array}{l}1.00 \pm \\
0.06\end{array}$ & $\begin{array}{c}4.70 \pm \\
0.28\end{array}$ & $\begin{array}{l}1.04 \pm \\
0.06\end{array}$ \\
\hline Median & 2.49 & 1.00 & 2.55 & 1.02 & 4.50 & 1.00 & 4.68 & 1.04 \\
\hline \multicolumn{9}{|l|}{ Exp. 2} \\
\hline $\begin{array}{r}\text { Mean } \pm \text { St. } \\
\text { deviation }\end{array}$ & $\begin{array}{c}1.46 \pm \\
0.32\end{array}$ & $\begin{array}{c}1.02 \pm \\
0.23\end{array}$ & $\begin{array}{c}1.72 \pm \\
0.34\end{array}$ & $\begin{array}{c}1.21 \pm \\
0.24\end{array}$ & $\begin{array}{c}3.67 \pm \\
1.01\end{array}$ & $\begin{array}{c}1.05 \pm \\
0.29\end{array}$ & $\begin{array}{c}3.16 \pm \\
1.61\end{array}$ & $\begin{array}{c}0.91 \pm \\
0.46\end{array}$ \\
\hline Median & 1.42 & 1.00 & 1.71 & 1.20 & 3.49 & 1.00 & 3.04 & 0.87 \\
\hline
\end{tabular}



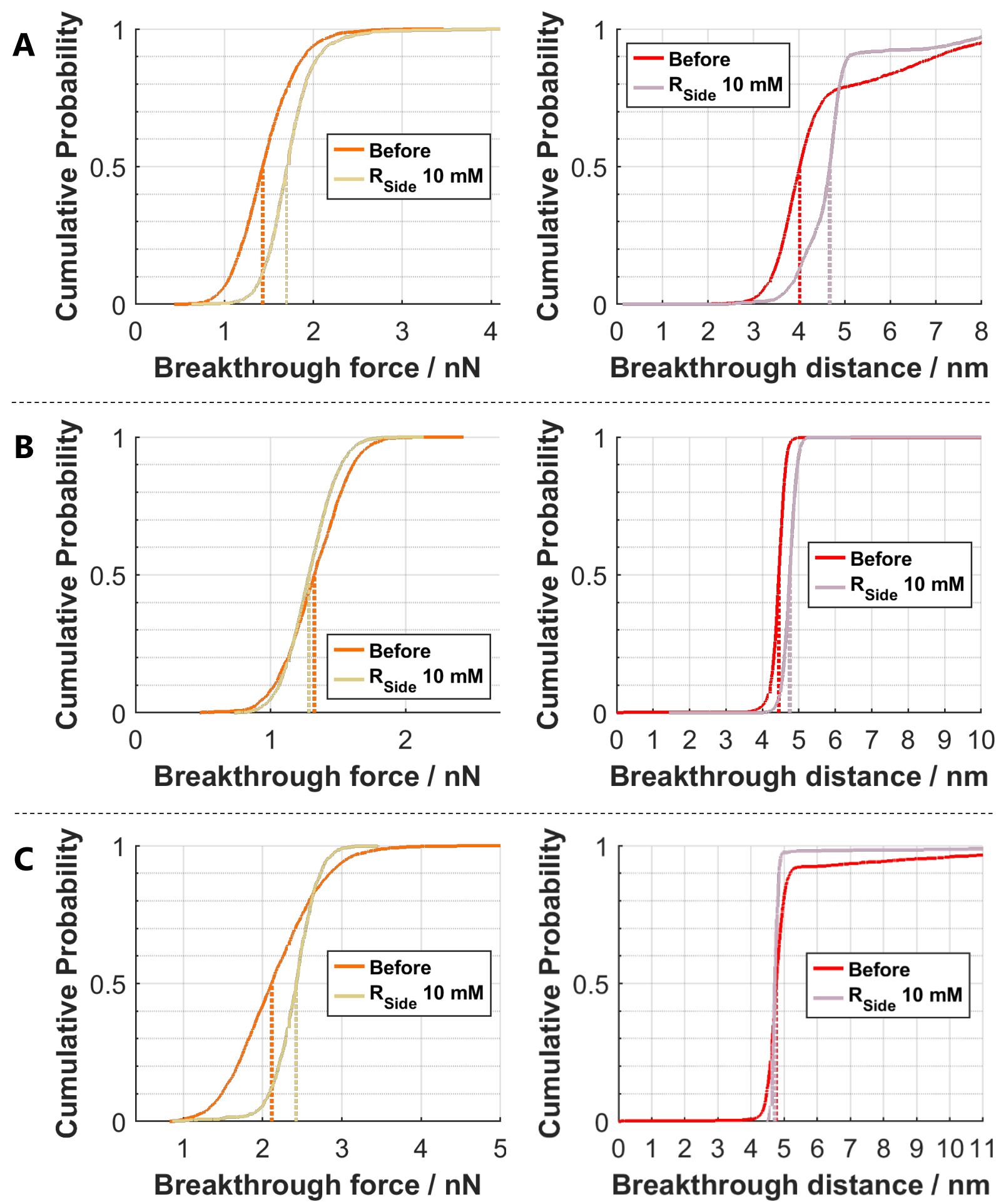

Figure A 4.2: Results of individual breakthrough force spectroscopy experiments on POPC:POPE:BP 49.5:49.5:1 membranes with $10 \mathrm{mM} \mathrm{N}$-Propylguanidine $\left(\mathrm{R}_{\text {Side }}\right)$. The left plots represent breakthrough force results, the right plots show breakthrough distance results. The part of the curves with the largest slope would be the peak in a histogram. Medians corresponding to this peak are indicated as dashed lines. A: Experiment 1; B: Experiment 2; C: Experiment 3. 
Table A 4.2: Means, medians and standard deviations to the datasets presented in the cumulative plots of Figure A 4.2.

\section{Breakthrough Force}

Before
$R_{\text {Side }} 10 \mathrm{mM}$

Exp. 1 Absolute/ $\mathrm{nN}$ Normalised/ Absolute/nN $\begin{gathered}\text { Normalised } \\ \text { a.u. }\end{gathered}$

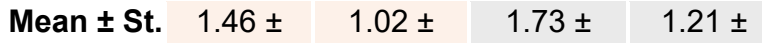

deviation 0.34

Median 1.43

Exp. 2

Mean \pm St. $1.32 \pm$

deviation 0.23

Median 1.32

Exp. 3

Mean \pm St

0.24

0.30

1.70

0.21

1.19

$1.00 \pm$

0.17

1.00

$1.29 \pm$

0.18

1.28

$0.97 \pm$

0.14

0.97

$2.41 \pm$

$1.14 \pm$

0.13

Median 2.12
0.28

2.42
1.15
Absolute / $\mathrm{nm}$

\section{Breakthrough Distance}

Before $\quad R_{\text {Side }} 10 \mathrm{mM}$

\begin{tabular}{|c|c|c|c|}
\hline $4.70 \pm$ & $1.17 \pm$ & $4.77 \pm$ & $1.19 \pm$ \\
\hline 3.07 & 0.76 & 1.07 & 0.27 \\
\hline 4.01 & 1.00 & 4.67 & 1.16 \\
\hline
\end{tabular}

\begin{tabular}{|l|c|c|c|}
\hline $4.44 \pm$ & $1.00 \pm$ & $4.74 \pm$ & $1.07 \pm$ \\
\hline 0.31 & 0.07 & 0.23 & 0.05 \\
\hline 4.45 & 1.00 & 4.75 & 1.07 \\
\hline
\end{tabular}

\begin{tabular}{|c|c|c|c|}
\hline $5.43 \pm$ & $1.14 \pm$ & $4.98 \pm$ & $1.05 \pm$ \\
\hline 3.63 & 0.76 & 2.78 & 0.58 \\
\hline 4.76 & 1.00 & 4.72 & 0.99 \\
\hline
\end{tabular}



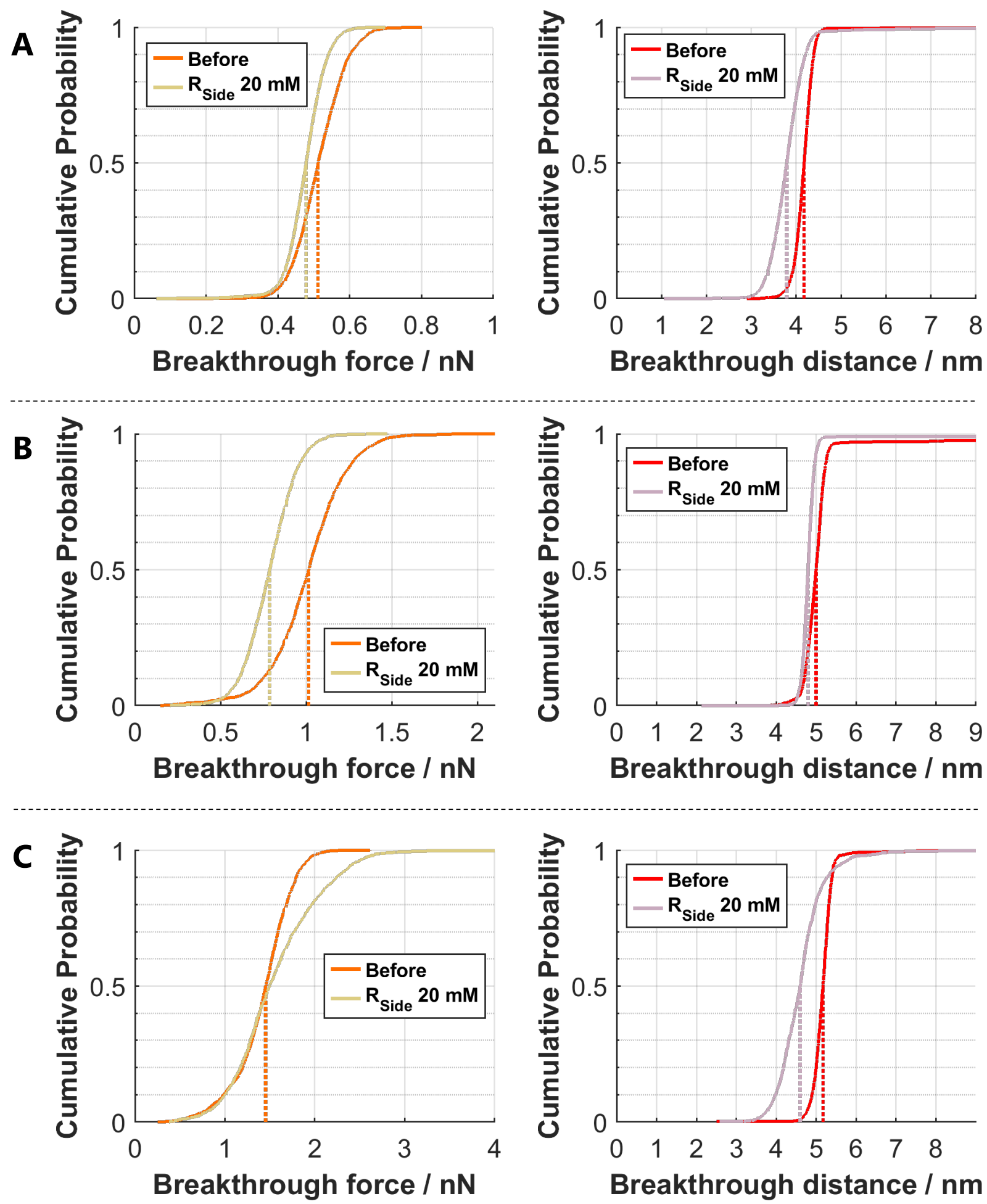

Figure A 4.3: Results of individual breakthrough force spectroscopy experiments on POPC:POPE:BP 49.5:49.5:1 membranes with $20 \mathrm{mM} \mathrm{N}$-Propylguanidine ( $R_{\text {side) }}$. The left plots represent breakthrough force results, the right plots show breakthrough distance results. The part of the curves with the largest slope would be the peak in a histogram. Medians corresponding to this peak are indicated as dashed lines. A: Experiment 1; B: Experiment 2; C: Experiment 3. 
Table A 4.3: Means, medians and standard deviations to the datasets presented in the cumulative plots of Figure A 4.3.

\section{Breakthrough Force}

Before
$\mathbf{R}_{\text {Side }} 20 \mathrm{mM}$

Exp. 1 Absolute $/ \mathrm{nN} \begin{gathered}\text { Normalised/ Absolute } / \mathrm{nN} \text { a.u. } \\ \text { a.u. }\end{gathered}$

\begin{tabular}{llll|l}
\hline Mean \pm St. & $0.51 \pm$ & $1.01 \pm$ & $0.48 \pm$ & $0.93 \pm$
\end{tabular} deviation 0.07

Median 0.51

Exp. 2

Mean \pm St. $1.01 \pm$

deviation 0.22

Median 1.02

Exp. 3

Mean \pm St.

0.32

Median 1.45

0.05

0.10

0.93

1.00

0.48

$0.99 \pm$

$0.79 \pm$

$0.78 \pm$

0.15

0.15

0.77

0.22
1.00

0.79

$0.98 \pm$

$1.35 \pm$

$0.93 \pm$

0.26

0.91
0.38

1.33
1.00

1.33

\section{Breakthrough Distance}

Before $\quad R_{\text {Side }} 20 \mathrm{mM}$

Absolute/nm Normalised/ Absolute/nm Normalise

\begin{tabular}{lll|l}
$4.17 \pm$ & $1.00 \pm$ & $3.83 \pm$ & $0.92 \pm$
\end{tabular}

\begin{tabular}{|l|l|l|l|}
\hline 0.39 & 0.09 & 0.86 & 0.21 \\
\hline
\end{tabular}

\begin{tabular}{|l|l|l|l|}
\hline 4.18 & 1.00 & 3.79 & 0.91 \\
\hline
\end{tabular}

\begin{tabular}{|c|c|c|c|}
\hline $5.03 \pm$ & $1.01 \pm$ & $4.80 \pm$ & $0.96 \pm$ \\
\hline 0.83 & 0.17 & 0.34 & 0.07 \\
\hline
\end{tabular}

\begin{tabular}{|l|l|l|l|}
\hline 4.99 & 1.00 & 4.79 & 0.96 \\
\hline
\end{tabular}

\begin{tabular}{|l|c|c|c|}
\hline $5.17 \pm$ & $1.00 \pm$ & $4.63 \pm$ & $0.90 \pm$ \\
\hline 0.35 & 0.07 & 0.61 & 0.12 \\
\hline 5.17 & 1.00 & 4.60 & 0.89
\end{tabular}



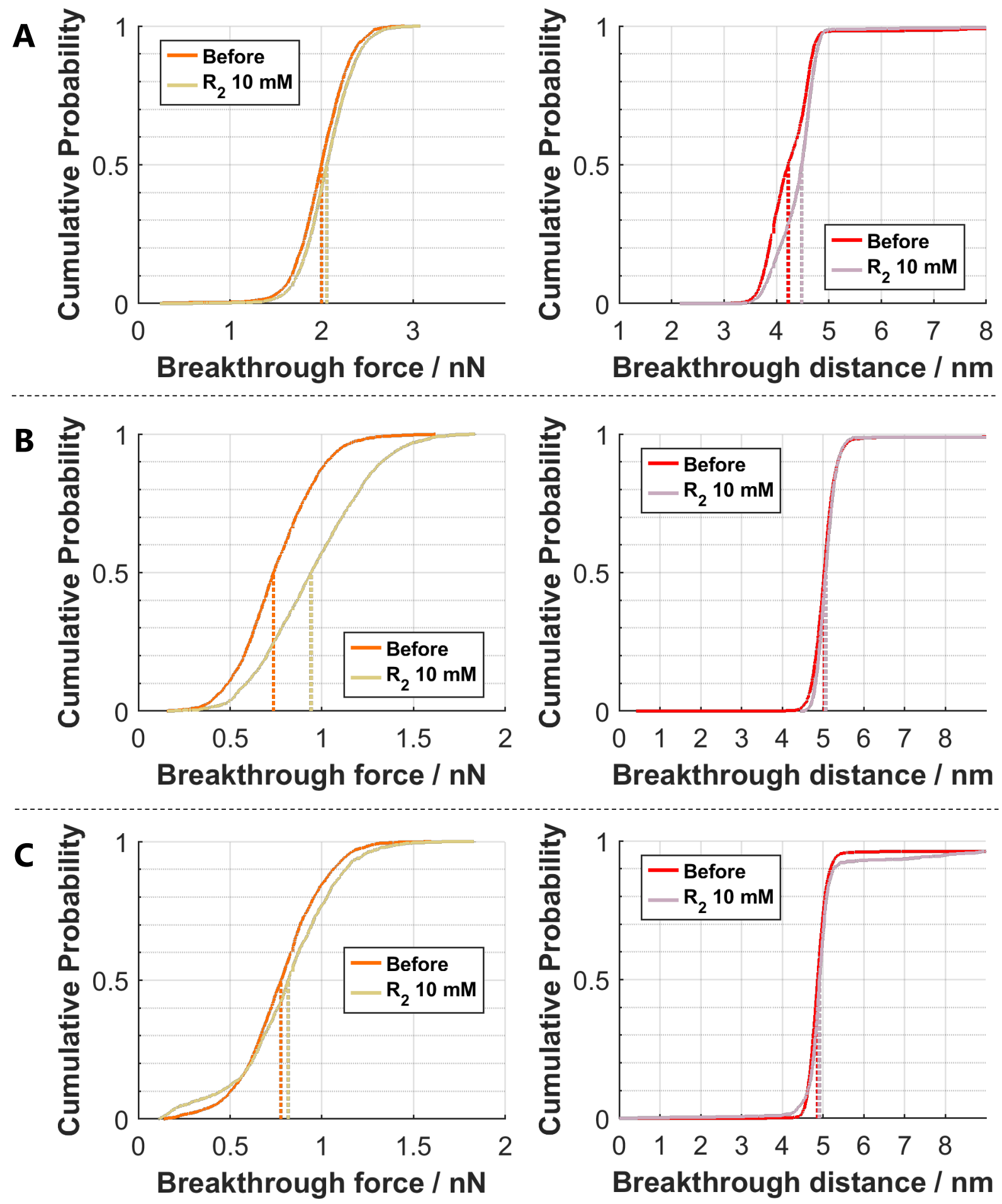

Figure A 4.4: Results of individual breakthrough force spectroscopy experiments on POPC:POPE:BP 49.5:49.5:1 membranes with $10 \mathrm{mM}$ of an arginine dipeptide $\left(R_{2}\right)$. The left plots represent breakthrough force results, the right plots show breakthrough distance results. The part of the curves with the largest slope would be the peak in a histogram. Medians corresponding to this peak are indicated as dashed lines. A: Experiment 1; B: Experiment 2; C: Experiment 3. 
Table A 4.4: Means, medians and standard deviations to the datasets presented in the cumulative plots of Figure A 4.4.

\section{Breakthrough Force}

Before

$\mathrm{R}_{\mathbf{2}} 10 \mathrm{mM}$

$\mathrm{R}_{\mathbf{2}} 10 \mathrm{mM}$

\begin{tabular}{rc} 
Exp. 1 & Absolute $/ \mathrm{nN}$ \\
\hline Mean \pm St. & $2.00 \pm$ \\
deviation & 0.28 \\
Median & 2.00
\end{tabular}

Exp. 2

\begin{tabular}{r|c|c|c|c|}
\hline Mean \pm St. & $0.75 \pm$ & $1.02 \pm$ & $0.95 \pm$ & $1.30 \pm$ \\
\hline deviation & 0.21 & 0.29 & 0.28 & $0.39 \mathrm{k}$ \\
\hline Median & 0.74 & 1.00 & 0.94 & 1.28
\end{tabular}

Exp. 3

Mean $\pm \mathrm{St}$

deviation 0.22

Median $\quad 0.78$

\section{Breakthrough Distance}

\section{Before $\quad R_{2} 10 \mathrm{mM}$}
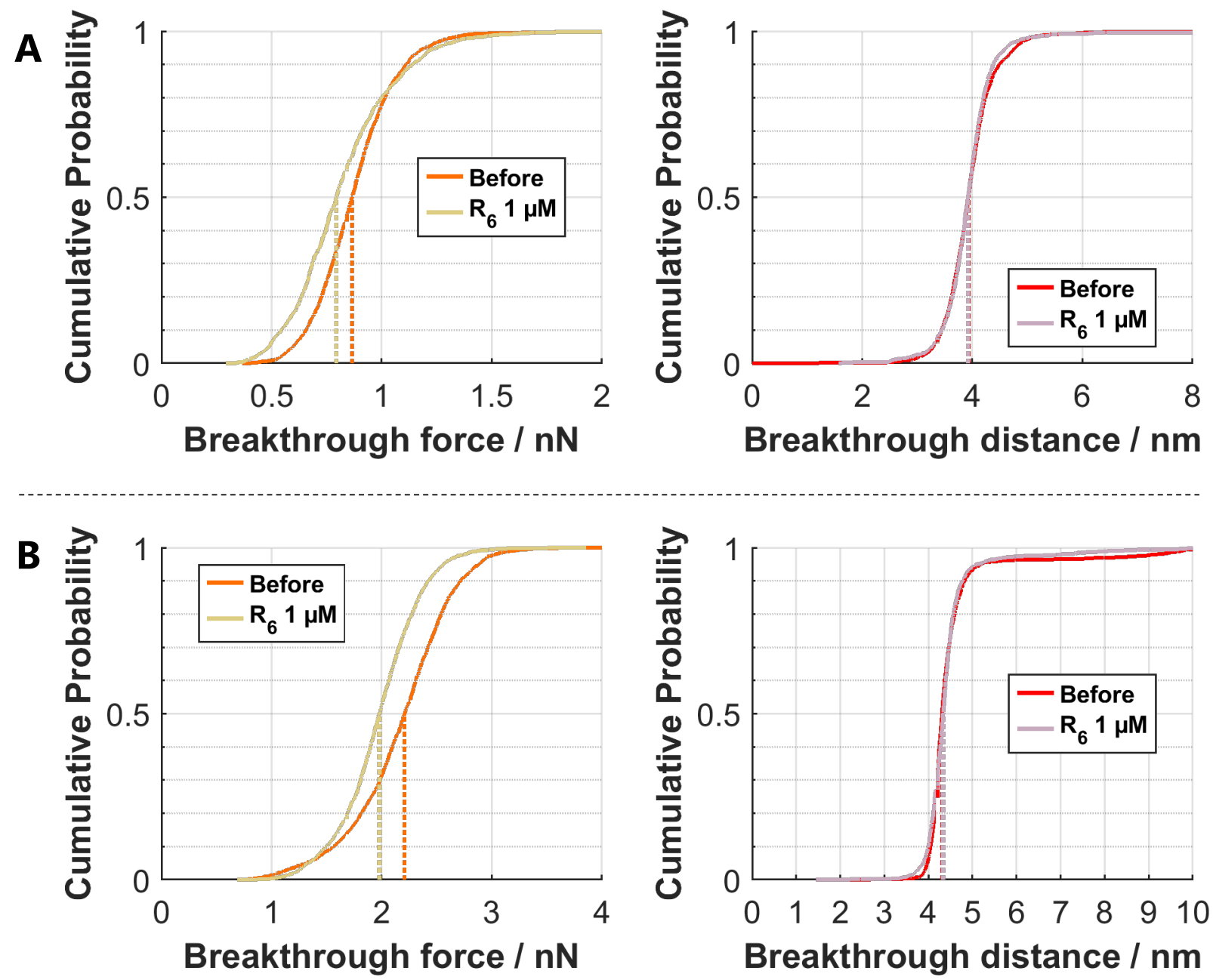

Figure A 4.5: Results of a breakthrough force spectroscopy experiment on POPC:POPE:BP 49.5:49.5:1 membranes, with $1 \mu \mathrm{M}$ of a hexa-arginine peptide $\left(R_{6}\right)$. The left plot presents the breakthrough force results, the right plot shows the breakthrough distance results. A: Experiment 1; B: Experiment 2; 
Table A 4.5: Means, medians and standard deviations to the datasets presented in the cumulative plots of Figure A 4.5 .

\section{Breakthrough Force}

Before
$R_{6} 1 \mu \mathrm{M}$

\section{Breakthrough Distance}

Before

\begin{tabular}{|c|c|c|c|c|c|c|c|c|}
\hline Exp. 1 & Absolute / $\mathrm{nN}$ & $\begin{array}{l}\text { Normalised / } \\
\text { a.u. }\end{array}$ & Absolute / nN & $\begin{array}{l}\text { Normalised / } \\
\text { a.u. }\end{array}$ & Absolute / nm & $\begin{array}{l}\text { Normalised / } \\
\text { a.u. }\end{array}$ & Absolute / nm & $\begin{array}{l}\text { Normalised/ } \\
\text { a.u. }\end{array}$ \\
\hline $\begin{array}{r}\text { Mean } \pm \text { St. } \\
\text { deviation }\end{array}$ & $\begin{array}{c}2.00 \pm \\
0.28\end{array}$ & $\begin{array}{c}1.00 \pm \\
0.14\end{array}$ & $\begin{array}{c}2.06 \pm \\
0.28\end{array}$ & $\begin{array}{c}1.03 \pm \\
0.14\end{array}$ & $\begin{array}{c}4.27 \pm \\
0.69\end{array}$ & $\begin{array}{l}1.01 \pm \\
0.16\end{array}$ & $\begin{array}{c}4.42 \pm \\
0.60\end{array}$ & $\begin{array}{c}1.05 \pm \\
0.14\end{array}$ \\
\hline Median & 2.00 & 1.00 & 2.06 & 1.03 & 4.22 & 1.00 & 4.48 & 1.06 \\
\hline \multicolumn{9}{|l|}{ Exp. 2} \\
\hline $\begin{array}{r}\text { Mean } \pm \text { St. } \\
\text { deviation }\end{array}$ & $\begin{array}{c}0.75 \pm \\
0.21\end{array}$ & $\begin{array}{c}1.02 \pm \\
0.29\end{array}$ & $\begin{array}{c}0.95 \pm \\
0.28\end{array}$ & $\begin{array}{l}1.30 \pm \\
0.39 \mathrm{k}\end{array}$ & $\begin{array}{c}5.07 \pm \\
0.78\end{array}$ & $\begin{array}{c}1.01 \pm \\
0.16\end{array}$ & $\begin{array}{c}5.12 \pm \\
0.77\end{array}$ & $\begin{array}{l}1.02 \pm \\
0.15\end{array}$ \\
\hline Median & 0.74 & 1.00 & 0.94 & 1.28 & 5.03 & 1.00 & 5.06 & 1.01 \\
\hline
\end{tabular}
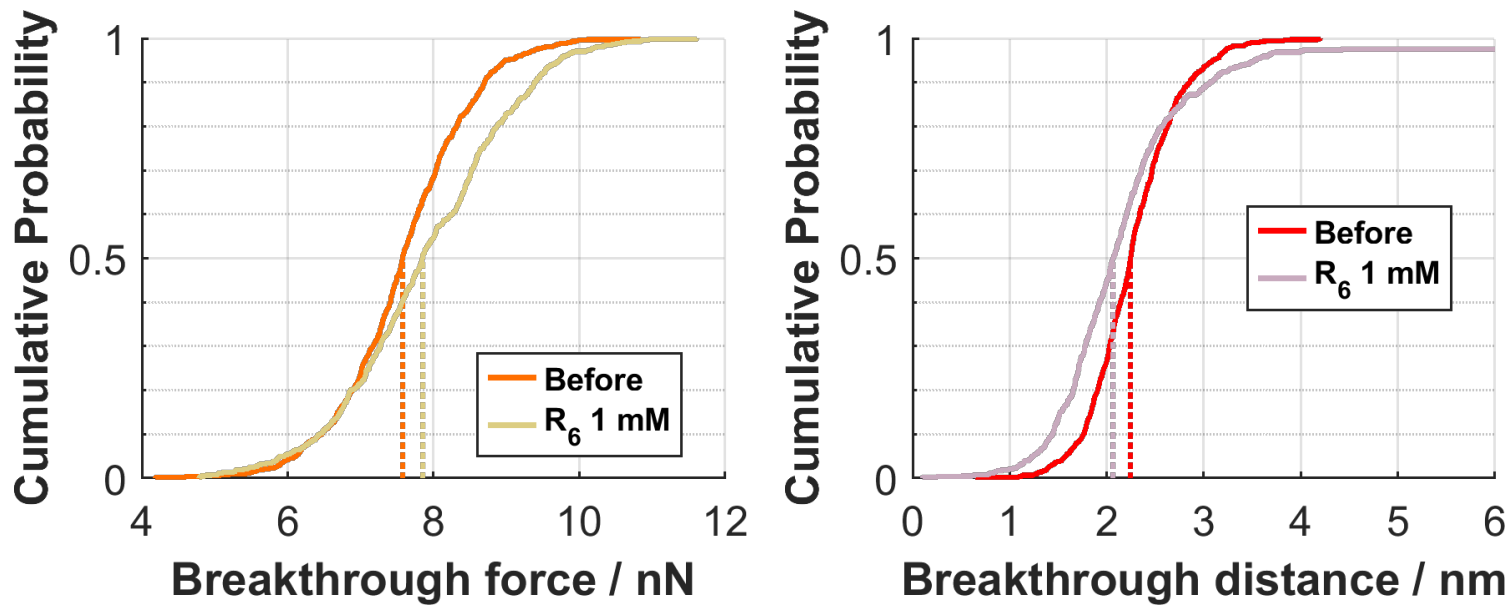

Figure A 4.6: Results of a breakthrough force spectroscopy experiment on POPC:POPE:BP 49.5:49.5:1 membrane, with $1 \mathrm{mM}$ of a hexa-arginine peptide $\left(R_{6}\right)$. The left plot presents the breakthrough force results, the right plot shows the breakthrough distance results. The part of the curves with the largest slope would be the peak in a histogram. Medians corresponding to this peak are indicated as dashed lines.

Table A 4.6: Means, medians and standard deviations to the datasets presented in the cumulative plots of Figure A 4.6. Absolute as well as normalised values are given. Normalisation was performed for the median of each dataset recorded on the lipid without peptide.

\section{Breakthrough Force}

\begin{tabular}{|c|c|c|c|c|c|c|c|c|}
\hline & \multicolumn{2}{|c|}{ Before } & \multicolumn{2}{|c|}{$\mathrm{R}_{6} 1 \mathrm{mM}$} & \multicolumn{2}{|c|}{ Before } & \multicolumn{2}{|c|}{$\mathrm{R}_{6} 1 \mathrm{mM}$} \\
\hline & Absolute / $\mathrm{nN}$ & $\begin{array}{l}\text { Normalised/I } \\
\text { a.u. }\end{array}$ & Absolute / $\mathrm{nN}$ & $\begin{array}{l}\text { Normalised / } \\
\text { a.u. }\end{array}$ & Absolute / nm & $\begin{array}{l}\text { Normalised/ } \\
\text { a.u. }\end{array}$ & Absolute / $\mathrm{nm}$ & $\begin{array}{l}\text { Normalised/ } \\
\text { a.u. }\end{array}$ \\
\hline $\begin{array}{r}\text { Mean } \pm \text { St. } \\
\text { deviation }\end{array}$ & $\begin{array}{c}7.59 \pm \\
0.91\end{array}$ & $\begin{array}{c}1.00 \pm \\
0.12\end{array}$ & $\begin{array}{c}7.89 \pm \\
1.16\end{array}$ & $\begin{array}{l}1.04 \pm \\
0.15\end{array}$ & $\begin{array}{c}2.27 \pm \\
0.46\end{array}$ & $\begin{array}{l}1.01 \pm \\
0.20\end{array}$ & $\begin{array}{c}2.14 \pm \\
0.76\end{array}$ & $\begin{array}{c}0.96 \pm \\
0.34\end{array}$ \\
\hline Median & 7.57 & 1.00 & 7.85 & 1.04 & 2.25 & 1.00 & 2.05 & 0.91 \\
\hline
\end{tabular}


Table A 5.1: Elaborate statistical quantities for the normalised breakthrough force results presented in Figure 4.3, Figure 4.5 and Figure 4.6.

\begin{tabular}{|c|c|c|c|c|c|c|c|}
\hline POPC & $\begin{array}{l}\text { Pure } \\
\text { Lipid }\end{array}$ & $\begin{array}{c}\mathrm{K} \\
10 \mathrm{mM}\end{array}$ & $\begin{array}{c}R_{\text {Side }} \\
10 \mathrm{mM}\end{array}$ & $\begin{array}{c}R_{\text {Side }} \\
20 \mathrm{mM}\end{array}$ & $\begin{array}{c}R_{2} \\
10 \mathrm{mM}\end{array}$ & $\begin{array}{c}R_{6} \\
1 \mu \mathrm{M}\end{array}$ & $\begin{array}{c}R_{6} \\
1 \mathrm{mM}\end{array}$ \\
\hline $\begin{array}{r}\text { Mean } \pm \text { St. } \\
\text { deviation }\end{array}$ & $\begin{array}{c}1.01 \pm \\
0.28\end{array}$ & $\begin{array}{c}0.92 \pm \\
0.13\end{array}$ & $\begin{array}{c}1.20 \pm \\
0.26\end{array}$ & $\begin{array}{c}1.47 \pm \\
0.34\end{array}$ & $\begin{array}{c}0.95 \pm \\
0.34\end{array}$ & $\begin{array}{c}1.05 \pm \\
0.14\end{array}$ & $\begin{array}{c}0.94 \pm \\
0.20\end{array}$ \\
\hline Median & 1.00 & 0.92 & 1.23 & 1.46 & 0.91 & 1.05 & 0.91 \\
\hline $\operatorname{StDev} / \sqrt{N}$ & 0.0017 & 0.0019 & 0.0027 & 0.0035 & 0.0027 & 0.0029 & 0.0044 \\
\hline Data Size & 28360 & 5120 & 9267 & 9545 & 15811 & 2304 & 2048 \\
\hline \# Outliers & 1273 & 65 & 634 & 90 & 982 & 6 & 18 \\
\hline$\%$ Outliers & 4.49 & 1.27 & 6.84 & 0.94 & 6.21 & 0.26 & 0.88 \\
\hline $\begin{array}{c}\text { POPC:POPG } \\
1: 1\end{array}$ & $\begin{array}{l}\text { Pure } \\
\text { Lipid }\end{array}$ & $\begin{array}{c}\mathrm{K} \\
10 \mathrm{mM}\end{array}$ & $\begin{array}{c}R_{\text {Side }} \\
10 \mathrm{mM}\end{array}$ & $\begin{array}{c}R_{\text {Side }} \\
20 \mathrm{mM}\end{array}$ & $\begin{array}{c}R_{2} \\
10 \mathrm{mM}\end{array}$ & $\begin{array}{c}R_{6} \\
1 \mu \mathrm{M}\end{array}$ & $\begin{array}{c}R_{6} \\
1 \mathrm{mM}\end{array}$ \\
\hline $\begin{array}{r}\text { Mean } \pm \text { St. } \\
\text { deviation }\end{array}$ & $\begin{array}{c}1.00 \pm \\
0.21\end{array}$ & $\begin{array}{c}1.06 \pm \\
0.20\end{array}$ & $\begin{array}{c}0.63 \pm \\
0.22\end{array}$ & $\begin{array}{c}0.58 \pm \\
0.16\end{array}$ & $\begin{array}{c}0.78 \pm \\
0.16\end{array}$ & $\begin{array}{l}1.04 \pm \\
0.15\end{array}$ & $\begin{array}{l}1.01 \pm \\
0.26\end{array}$ \\
\hline Median & 1.00 & 1.06 & 0.60 & 0.60 & 0.77 & 1.02 & 0.95 \\
\hline $\operatorname{StDev} / \sqrt{N}$ & 0.0011 & 0.0028 & 0.0026 & 0.0014 & 0.0017 & 0.0034 & 0.0044 \\
\hline Data Size & 35545 & 5112 & 7471 & 14217 & 8417 & 2048 & 3584 \\
\hline \# Outliers & 1034 & 77 & 494 & 115 & 118 & 56 & 15 \\
\hline$\%$ Outliers & 2.91 & 1.51 & 6.61 & 0.81 & 1.40 & 2.73 & 0.42 \\
\hline $\begin{array}{c}\text { POPC:POPE } \\
1: 1\end{array}$ & $\begin{array}{l}\text { Pure } \\
\text { Lipid }\end{array}$ & $\begin{array}{c}\mathrm{K} \\
10 \mathrm{mM}\end{array}$ & $\begin{array}{c}R_{\text {Side }} \\
10 \mathrm{mM}\end{array}$ & $\begin{array}{c}R_{\text {Side }} \\
20 \mathrm{mM}\end{array}$ & $\begin{array}{c}R_{2} \\
10 \mathrm{mM}\end{array}$ & $\begin{array}{c}R_{6} \\
1 \mu \mathrm{M}\end{array}$ & $\begin{array}{c}R_{6} \\
1 \mathrm{mM}\end{array}$ \\
\hline $\begin{array}{r}\text { Mean } \pm \text { St. } \\
\text { deviation }\end{array}$ & $\begin{array}{c}1.01 \pm \\
0.22\end{array}$ & $\begin{array}{c}1.12 \pm \\
0.21\end{array}$ & $\begin{array}{c}1.04 \pm \\
0.18\end{array}$ & $\begin{array}{c}0.90 \pm \\
0.15\end{array}$ & $\begin{array}{c}1.09 \pm \\
0.28\end{array}$ & $\begin{array}{c}0.92 \pm \\
0.22\end{array}$ & $\begin{array}{l}1.04 \pm \\
0.15\end{array}$ \\
\hline Median & 1.00 & 1.09 & 1.02 & 0.91 & 1.06 & 0.90 & 1.04 \\
\hline StDev $/ \sqrt{N}$ & 0.0012 & 0.0051 & 0.0018 & 0.0016 & 0.0030 & 0.0038 & 0.0062 \\
\hline Data Size & 31394 & 1792 & 10238 & 9138 & 8636 & 3326 & 610 \\
\hline \# Outliers & 611 & 29 & 118 & 284 & 629 & 66 & 1 \\
\hline$\%$ Outliers & 1.95 & 1.62 & 1.15 & 3.11 & 7.28 & 1.98 & 0.16 \\
\hline
\end{tabular}


Table A 5.2: Elaborate statistical quantities for the normalised breakthrough force results presented in Figure 4.8.

\begin{tabular}{|c|c|c|c|c|c|c|c|}
\hline POPC & $\begin{array}{l}\text { Pure } \\
\text { Lipid }\end{array}$ & $\begin{array}{c}\mathrm{K} \\
10 \mathrm{mM}\end{array}$ & $\begin{array}{c}R_{\text {Side }} \\
10 \mathrm{mM}\end{array}$ & $\begin{array}{c}R_{\text {Side }} \\
20 \mathrm{mM}\end{array}$ & $\begin{array}{c}R_{2} \\
10 \mathrm{mM}\end{array}$ & $\begin{array}{c}\mathbf{R}_{6} \\
1 \mu \mathrm{M}\end{array}$ & $\begin{array}{c}\mathbf{R}_{6} \\
1 \mathrm{mM}\end{array}$ \\
\hline $\begin{array}{l}\text { Mean } \pm \text { St. } \\
\text { deviation }\end{array}$ & $\begin{array}{c}1.00 \pm \\
0.21\end{array}$ & $\begin{array}{c}1.00 \pm \\
0.11\end{array}$ & $\begin{array}{c}0.99 \pm \\
0.33\end{array}$ & $\begin{array}{c}0.86 \pm \\
0.18\end{array}$ & $\begin{array}{c}1.00 \pm \\
0.14\end{array}$ & $\begin{array}{c}1.02 \pm \\
0.09\end{array}$ & $\begin{array}{c}0.94 \pm \\
0.11\end{array}$ \\
\hline Median & 1.00 & 0.99 & 0.98 & 0.87 & 1.00 & 1.03 & 0.95 \\
\hline StDev $/ \sqrt{N}$ & 0.0012 & 0.0015 & 0.0039 & 0.0018 & 0.0011 & 0.0020 & 0.0024 \\
\hline Data Size & 28360 & 5120 & 6907 & 9545 & 15811 & 2304 & 2048 \\
\hline \# Outliers & 2326 & 107 & 1540 & 92 & 1189 & 39 & 18 \\
\hline$\%$ Outliers & 8.20 & 2.09 & 22.30 & 0.96 & 7.52 & 1.69 & 0.88 \\
\hline $\begin{array}{c}\text { POPC:POPG } \\
1: 1\end{array}$ & $\begin{array}{l}\text { Pure } \\
\text { Lipid }\end{array}$ & $\begin{array}{c}\mathrm{K} \\
10 \mathrm{mM}\end{array}$ & $\begin{array}{c}R_{\text {Side }} \\
10 \mathrm{mM}\end{array}$ & $\begin{array}{c}R_{\text {Side }} \\
20 \mathrm{mM}\end{array}$ & $\begin{array}{c}R_{2} \\
10 \mathrm{mM}\end{array}$ & $\begin{array}{c}R_{6} \\
1 \mu \mathrm{M}\end{array}$ & $\begin{array}{c}R_{6} \\
1 \mathrm{mM}\end{array}$ \\
\hline $\begin{array}{c}\text { Mean } \pm \text { St. } \\
\text { deviation }\end{array}$ & $\begin{array}{c}1.02 \pm \\
0.21\end{array}$ & $\begin{array}{c}0.97 \pm \\
0.21\end{array}$ & $\begin{array}{c}1.06 \pm \\
0.39\end{array}$ & $\begin{array}{c}0.95 \pm \\
0.21\end{array}$ & $\begin{array}{c}0.99 \pm \\
0.18\end{array}$ & $\begin{array}{c}0.97 \pm \\
0.17\end{array}$ & $\begin{array}{c}0.89 \pm \\
0.22\end{array}$ \\
\hline Median & 1.00 & 0.98 & 1.01 & 0.94 & 1.02 & 0.96 & 0.84 \\
\hline StDev $/ \sqrt{N}$ & 0.0011 & 0.0015 & 0.0059 & 0.0019 & 0.0019 & 0.0038 & 0.0037 \\
\hline Data Size & 35036 & 5112 & 4337 & 12037 & 8417 & 2048 & 3584 \\
\hline \# Outliers & 3271 & 545 & 310 & 151 & 790 & 57 & 110 \\
\hline$\%$ Outliers & 9.34 & 10.66 & 7.15 & 1.25 & 9.39 & 2.78 & 3.07 \\
\hline $\begin{array}{c}\text { POPC:POPE } \\
1: 1\end{array}$ & $\begin{array}{l}\text { Pure } \\
\text { Lipid }\end{array}$ & $\begin{array}{c}\mathrm{K} \\
10 \mathrm{mM}\end{array}$ & $\begin{array}{c}R_{\text {Side }} \\
10 \mathrm{mM}\end{array}$ & $\begin{array}{c}R_{\text {Side }} \\
20 \mathrm{mM}\end{array}$ & $\begin{array}{c}R_{2} \\
10 \mathrm{mM}\end{array}$ & $\begin{array}{c}R_{6} \\
1 \mu \mathrm{M}\end{array}$ & $\begin{array}{c}R_{6} \\
1 \mathrm{mM}\end{array}$ \\
\hline $\begin{array}{c}\text { Mean } \pm \text { St. } \\
\text { deviation }\end{array}$ & $\begin{array}{c}1.01 \pm \\
0.22\end{array}$ & $\begin{array}{c}1.12 \pm \\
0.21\end{array}$ & $\begin{array}{c}1.04 \pm \\
0.18\end{array}$ & $\begin{array}{c}0.90 \pm \\
0.15\end{array}$ & $\begin{array}{c}1.09 \pm \\
0.28\end{array}$ & $\begin{array}{c}0.92 \pm \\
0.22\end{array}$ & $\begin{array}{c}1.04 \pm \\
0.15\end{array}$ \\
\hline Median & 1.00 & 1.09 & 1.02 & 0.91 & 1.06 & 0.90 & 1.04 \\
\hline StDev $/ \sqrt{N}$ & 0.0012 & 0.0051 & 0.0018 & 0.0016 & 0.0030 & 0.0038 & 0.0062 \\
\hline Data Size & 31394 & 1792 & 10238 & 9138 & 8636 & 3326 & 610 \\
\hline \# Outliers & 611 & 29 & 118 & 284 & 629 & 66 & 1 \\
\hline$\%$ Outliers & 1.95 & 1.62 & 1.15 & 3.11 & 7.28 & 1.98 & 0.16 \\
\hline
\end{tabular}


6. Leakage assay of POPG-containing lipid vesicles with $\mathrm{R}_{6}$ and carboxyfluorescein

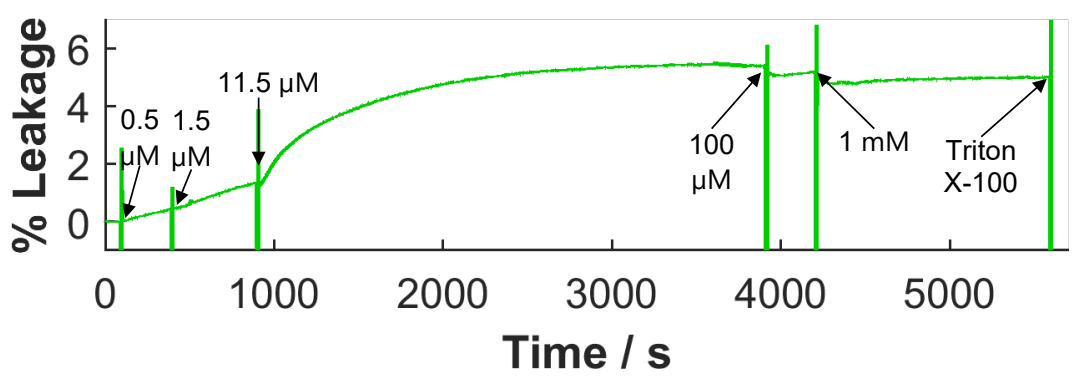

Figure A 6.5.1: Leakage assay for $R_{6}$ in Hepes buffer with carboxyfluorescein as a dye $\left(\lambda_{\mathrm{em}}=520 \mathrm{~nm}\right.$, $\lambda_{\mathrm{ex}}=490 \mathrm{~nm}$ ), in POPG-containing vesicles. Lipid concentration of POPC:POPG:BP 49.5:49.5:1 vesicles was calculated to be $16.2 \mu \mathrm{M}$ lipids or $0.5 \mu \mathrm{g}$ phosphorus per $\mathrm{mL}$. Data were corrected for linear increase by subtracting a line fitted through the fluorescence data without $R_{6}$ present. Normalisation was performed to $0 \%$ for data without $R_{6}$, and $100 \%$ for fluorescence intensity after Triton X-100 addition. Peptide-tolipid ratios ranged from 1:32 for $0.5 \mu \mathrm{M} \mathrm{R}_{6}$, to $63: 1$ for $1 \mathrm{mM} \mathrm{R}_{6}$. At $10 \mu \mathrm{M}$, where some leakage is detected, it corresponded to P:L 1:1.38. Total leakage was ca. 5.4\%.

For this assay, a Hepes buffer was used: $10 \mathrm{mM}$ Hepes, $100 \mathrm{mM} \mathrm{NaCl}$, pH 7.4. 5(6)-Carboxyfluorescein (-xanthene]-5-carboxylicacid) was dissolved to a $100 \mathrm{mM}$ stock solution in Hepes buffer. The solution was stored at $4{ }^{\circ} \mathrm{C}$ before use. A $2 \mathrm{mg}$ lipid film of the desired composition was hydrated with $400 \mu \mathrm{L}$ of this solution, and vesicles were prepared according to the procedure described in Materials \& Methods $p$. 53. The concentration of lipids in the vesicle solution was determined by phosphate test. Absorbance measurements for this test were performed on a Jasco UV-Vis spectrophotometer. For the assay, the vesicle solution was diluted to $0.001 \mathrm{mg} / \mathrm{mL}$ in measuring buffer as calculated from the determined concentration of lipids in stock solution. 


\section{Exemplary data of FRAP on POPG-containing bilayers in the presence of $\mathrm{R}_{\text {side }}$}
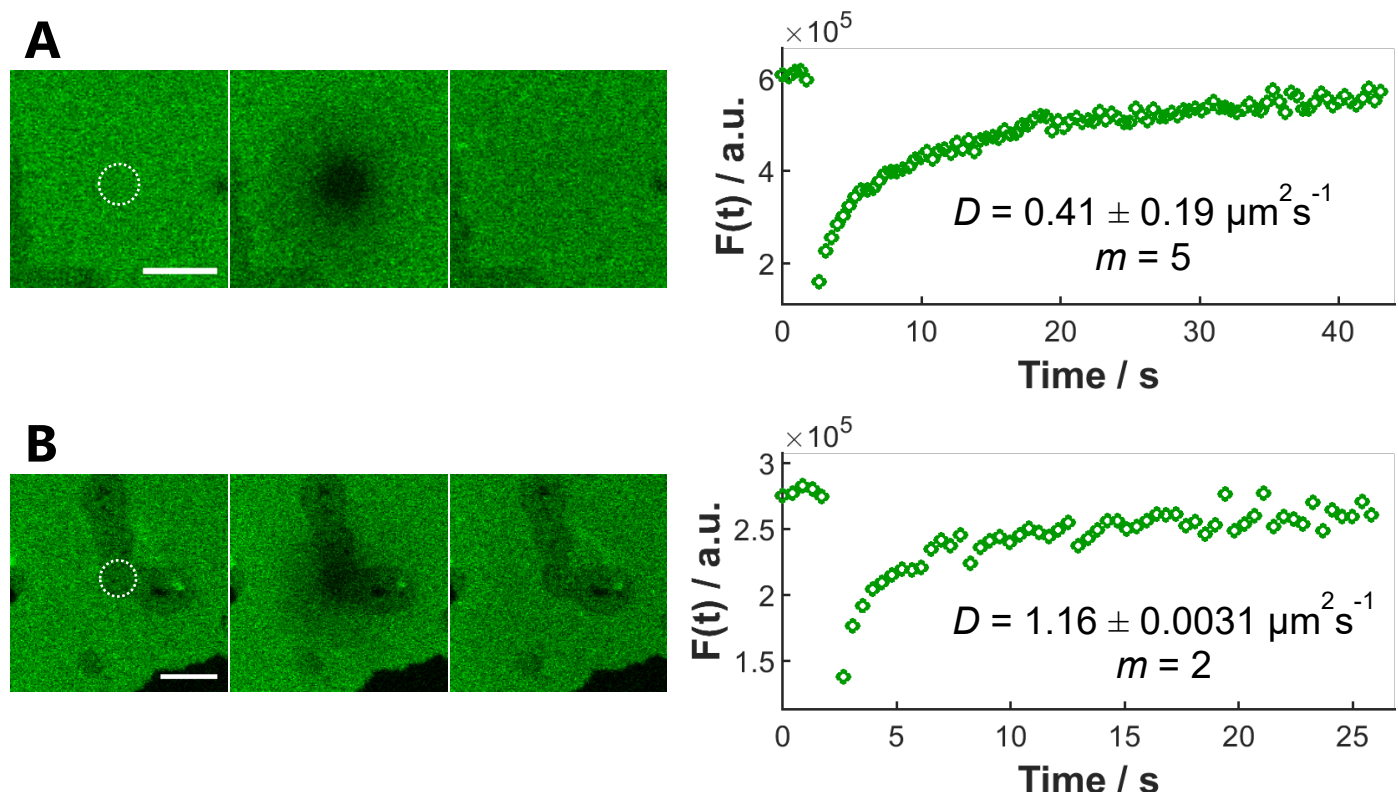

Figure A 6.1: Exemplary data of the diffusion coefficient of BP as measured on the global membrane (A) and the darker areas in the presence of $\mathrm{R}_{\text {side }}(\mathbf{B})$. To the left, exemplary fluorescence micrographs of a FRAP experiment are shown. On the left, an example of total fluorescence as measured within the dashed circle on the micrographs is presented, with the deduced diffusion coefficients and number of measurements $m$ performed. Despite the values differing by about a factor 2 , this difference was not deemed significant; the size of the dark spots was too small to bleach accurately, and the incomplete bleaching could also have accounted for the observed difference. 
8. RIfS expirements on 50\% POPS membranes with vesicles present

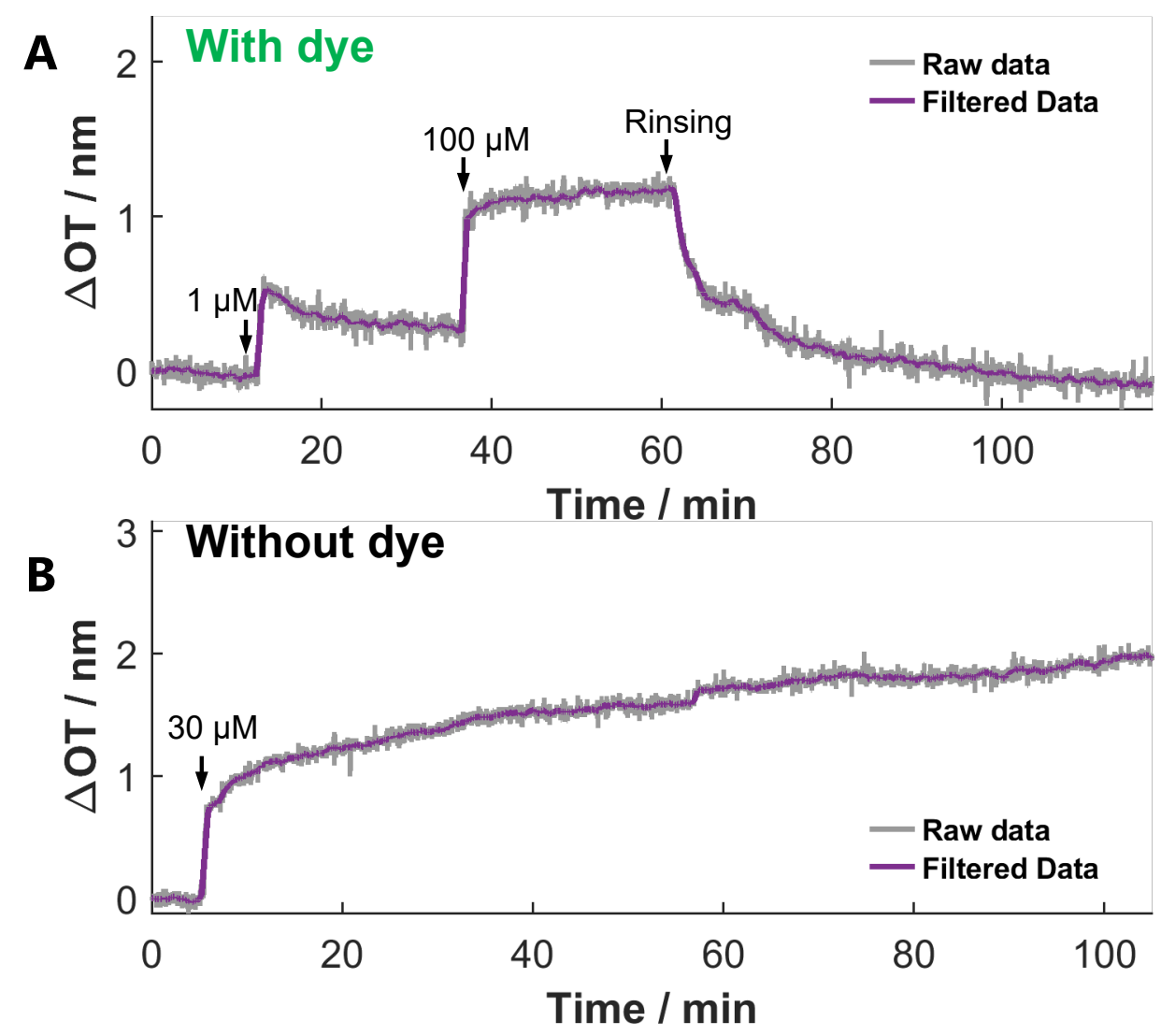

Figure A 8.1: RIfS experiments with $R_{6}$ addition to POPC:POPS 1:1 membranes in the presence of vesicles (due to closed system bilayer rinsing). Optical thickness is shown after bilayer formation and closed system rinsing. $R_{6}$ concentrations as well as afterwards rinsing are indicated in each figure. A: $1 \%$ Bodipy dye; $\mathbf{B}$ : Without dye. 


\section{Bleach profiles for stack and main membrane}

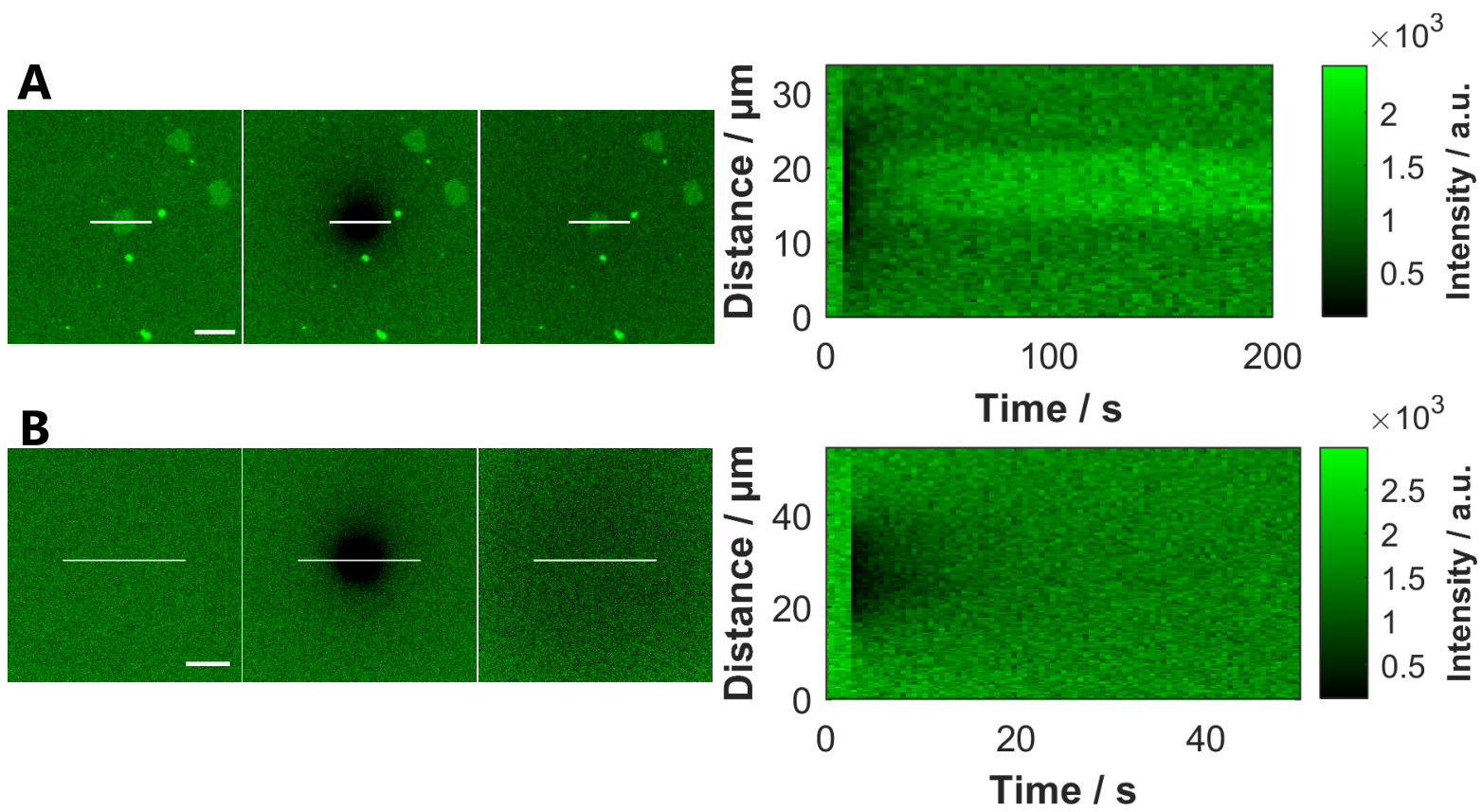

Figure A 9.1: Bleach profiles (right) of a cross-section through the bleached spot (indicated on left images of a POPC:POPS:BP 49.5:49.5:1 membrane before bleaching, after bleaching and after recovery). A: Profile of a membrane stack, uniform symmetric recovery; B: Recovery profile of an exemplary FRAP experiment on the main membrane. 


\section{Lower stability of second level membrane stacks}

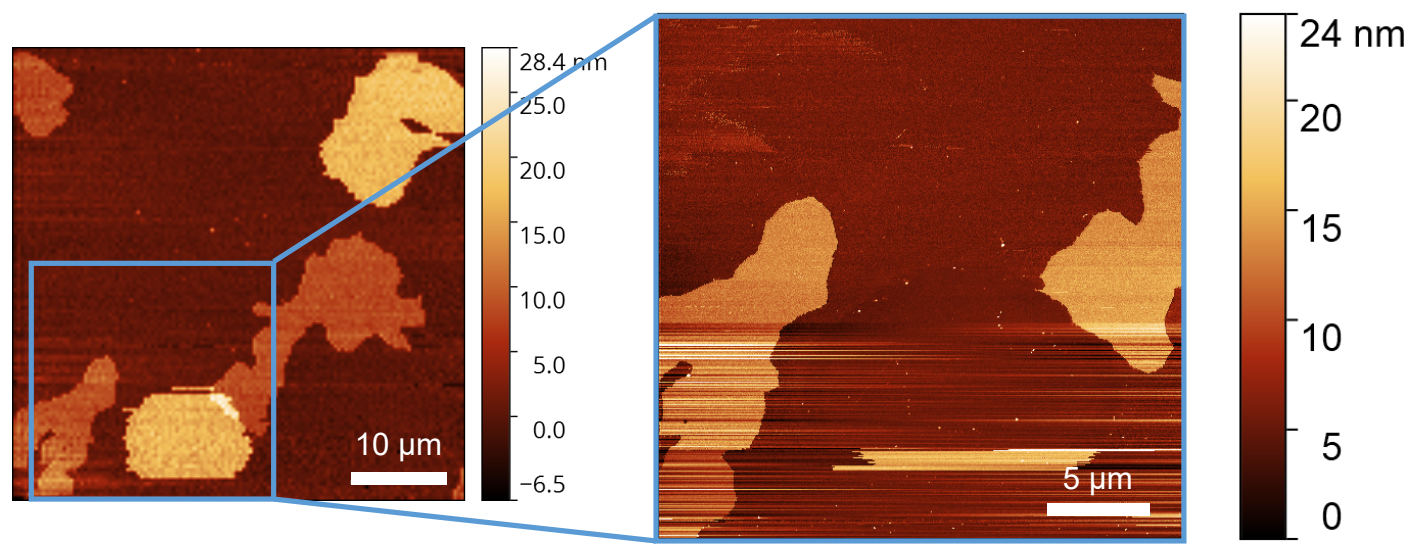

Figure A 10.1: $\mathrm{Q}^{\mathrm{TM}}$ AFM image zoomed in on Figure 5.1 B $p .92$ (repeated to the left). The second level stack destabilises upon higher resolution imaging, while the first level structures around it stay intact (right). 
11. Supplementary fluorescence micrographs
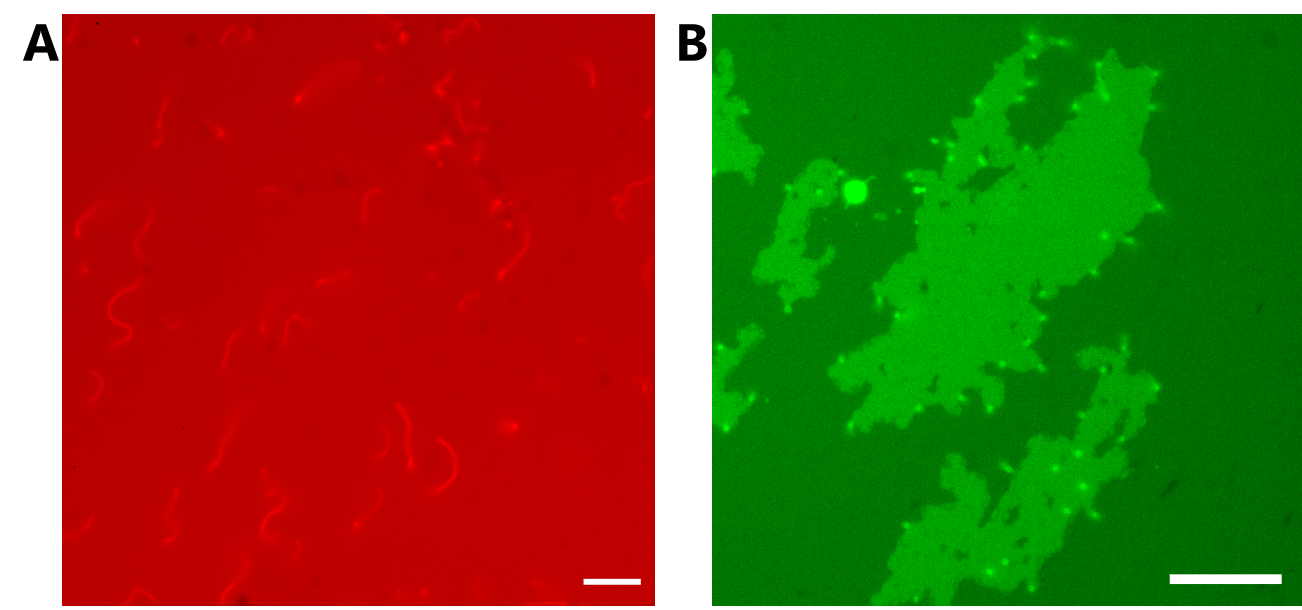

Figure A 11.1 Supplementary fluorescence micrographs. A: POPG-containing membrane stained with Rh18 in the absence of R-peptide displays excessive tubular structures, similar as with Bodipy dye. B: $R_{6}$-induced stacks on POPG-containing bilayers also display tubular structures at their borders, similarly to POPS-containing membranes in Figure 5.9 A p. 100.

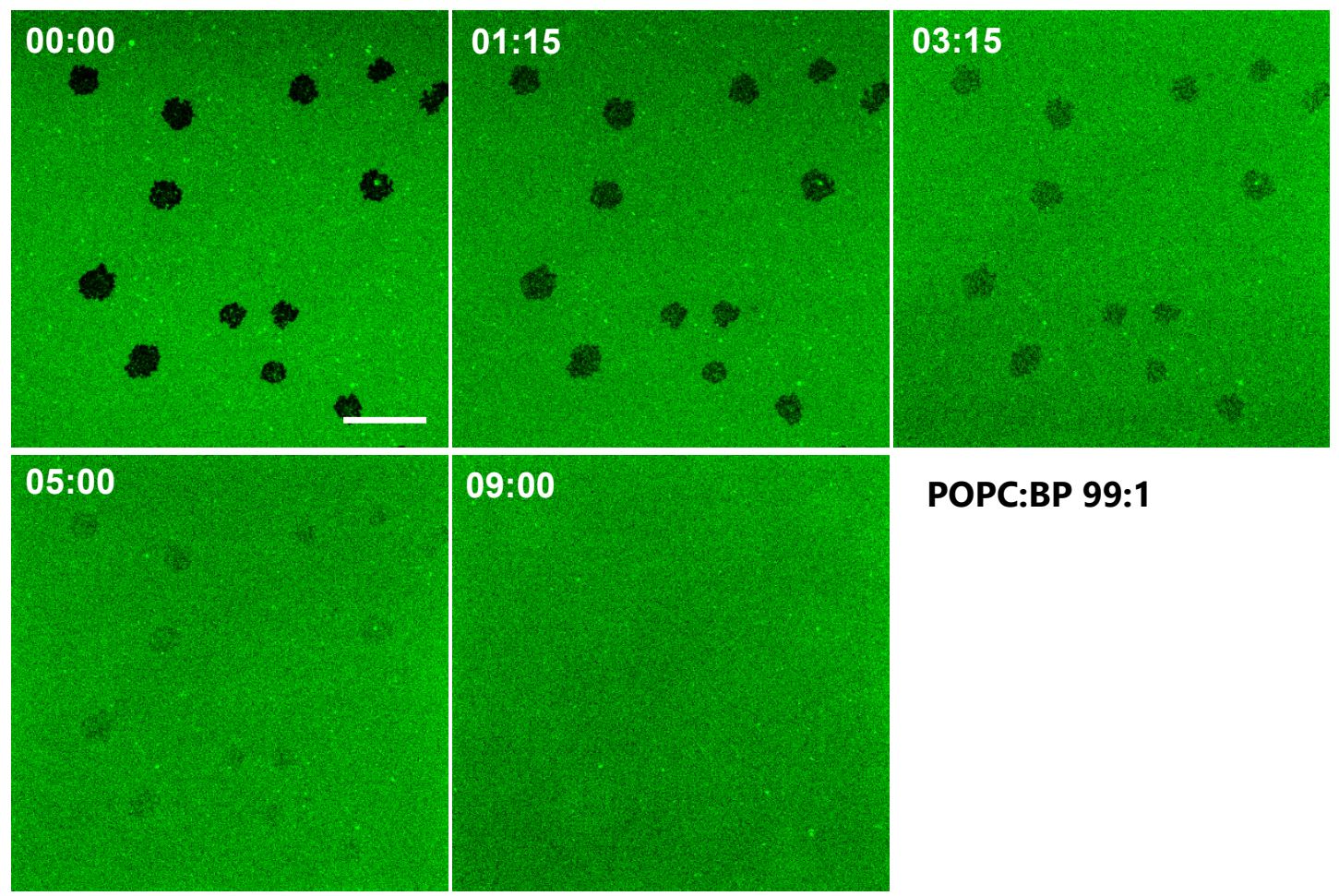

Figure A 11.2: Anecdotal observation of temporary dark domains for POPC:BP 99:1 membranes briefly after addition of $20 \mathrm{mM}$ Rside. Time in mm:ss, scale bar $20 \mu \mathrm{m}$. 


\section{List of Figures}

Figure 1.1: Basic amino acids L-arginine (A) and L-lysine (B) in neutral form.

Figure 1.2: Generally proposed mechanisms of entry for CPPs.

Figure 1.3: Proposed fatty acid- and $\mathrm{pH}$ gradient-mediated cell entry mechanisms for arginine-rich CPPs by Herce et al. [10]..

Figure 1.4: Mechanism of oligo-arginine entry via lamellarisation and fusion as proposed by Allolio et al. [11].

Figure 1.5: Chemical structure of molecular N-propylguanidine. A solution of protonated $\mathrm{N}$-propylguanidine is used as mimic of the arginine sidechain $\left(\mathrm{R}_{\text {side }}\right)$ in this work. 24

Figure 2.1: Principles of atomic force microscopy. 30

Figure 2.2: Illustration of an approach (red) and retract (blue) force vs. distance curve with a breakthrough event. 33

Figure 2.3: Illustration of cantilever tip radius $R$ and indentation depth $\delta$. 35

Figure 2.4: Concepts of reflectometry. 38

Figure 2.5: Principle of confocal laser scanning microscopy 40

Figure 2.6: Illustration of the deduction of pore nucleation energy from MD simulations of pore formation by Dr. Neha Awasthi adapted with permission with blinded out numbers and units.

Figure 3.1: Lipids used for model membranes in this work. 44

Figure 3.2: Chemical structures of membrane dyes used in this work. Manufacturer names for each dye are indicated, as well as colloquial abbreviations for these dyes used in this work. 45

Figure 3.3: Chemical structure of Sulforhodamine B sodium salt. 46

Figure 3.4: Pictures of Teflon sample chambers for solid-supported lipid bilayers.

Figure 3.5: Illustration of a breakthrough event in an indentation force curve as processed by the script written by Dr. Ingo Mey. 54

Figure 3.6: Schematic illustration of a closed and an open system for the flow cells in RIfS experiments. The yellow star signifies the light source and spectrometer. 
Figure 3.7 Illustration of the derivation of the mean time constant $\mathrm{T}$ from integrated fluorescence intensity data from a FRAP experiment.

Figure 4.1: Molecular Dynamics simulation results for pore formation in bilayers composed of either POPG, POPE or POPC, combined with arginine derivatives $\mathrm{R}_{2}, \mathrm{R}_{2}$ cap , and $\mathrm{R}_{\text {Side }}$

Figure 4.2: Exemplary breakthrough force spectroscopy results for all lipid investigated compositions.

Figure 4.3: Boxplots of normalised breakthrough force results for POPC:BP 99:1

Figure 4.4: Normalised boxplot results of a breakthrough force experiment during which the sample buffer was exchanged for one with a higher $\mathrm{pH}$ (6.3 to 7.3).

Figure 4.5: Boxplots of normalised breakthrough force results for POPC:POPE:BP 49.5:49.5:1

Figure 4.6: Breakthrough force results for POPC:POPG:BP 49.5:49.5:1 lipid mixtures combined with R-derivatives.

Figure 4.7: Exemplary initial force-separation curves of POPG-containing lipid bilayers with several concentrations of $R_{6}$, Colours serve to discriminate different force curves.

Figure 4.8: Boxplots of breakthrough distance results for all lipids and all Rderivatives.

Figure 4.9: Leakage assays of sulforhodamine B in vesicles of three different compositions.

Figure 4.10: Diffusion constants of BP for all lipids, as well as each lipid combined with all investigated R-derivatives.

Figure 4.11: Exemplary fluorescence micrographs of all lipid compositions. ... 80

Figure 4.12 A: Series of fluorescence micrographs of a POPC:POPG:BP 49.5:49.5:1 membrane recorded briefly after $\mathrm{R}_{\text {side-addition }}$

Figure 4.13: Plot of optical thickness against time, for a RIfS experiment on a POPC:POPG:BP 49.5:49.5:1 membrane with increasing concentrations of $\mathrm{R}_{\text {Side. }}$

Figure 4.14: Exemplary fluorescence micrograph series of a POPC:POPE:BP 49.5:49.5:1 membrane recorded after $\mathrm{R}_{\text {side }}$ addition $(10 \mathrm{mM}) \ldots 83$ 
Figure 4.15 Exemplary fluorescence micrographs of brighter patches occurring on POPC:POPG:BP 49.5:49.5:1 membranes under influence of $R_{6}$.

Figure 4.16: Two models to explain the fluorescence observations for $\mathrm{R}_{\text {side }}$ on lipid bilayers. Model 1: Rside causes local phase separation in the planar membrane by facilitating tighter lipid packing, and these tightly packed domains are devoid of dye.

Figure 5.1: Fluorescence micrograph (A) and AFM image (B) of the same section of a POPG-containing membrane in the presence of 1 $\mu \mathrm{M} \mathrm{R}_{6}$.

Figure 5.2: Mapped breakthrough force results of a POPC:POPG:BP 49.5:49.5:1 membrane in the presence of $1 \mathrm{mM} \mathrm{R}_{6}$.

Figure 5.3: Examples of FRAP experiments on membrane stacks of level 1 (A) and level 2 (B)

Figure 5.4 A: Illustration of the derivation of the mean time constant $T$ (green shaded area) from a FRAP experiment;

Figure 5.5: Fluorescence micrograph series on a membrane of composition POPC:POPS:BP 49.5:49.5:1, one frame before R-peptide addition and the first seven minutes after R-peptide addition. Elapsed time in seconds is indicated on the top left of each frame.

Figure 5.6: Stack growth analysis briefly after $\mathrm{R}_{6}$ addition (illumination only when recording images every $10 \mathrm{~s}$ ).

Figure 5.7: Stack area analysis on a POPG-containing membrane, an hour after R-peptide addition, under continuous epifluorescence illumination.

Figure 5.8: Zoomed in fluorescence micrograph series of the last seven frames of Stack 1 also shown in Figure 5.7 99

Figure 5.9: Two fluorescence micrograph series on POPC:POPS:BP 49.5:49.5:1 membranes, illustrating dynamic behaviour of stack growth and shrinkage in relationship to tether formation and vesicle budding.

Figure 5.10: Results for a POPC:POPG 1:1 membrane with an added vesicle population with a different dye, and subsequent addition of 1 $\mu \mathrm{m} \mathrm{R}_{6}$. 
Figure 5.11: Closed system RIfS experiments with $\mathrm{R}_{6}$ addition to POPC:POPG 1:1 membranes. Optical thickness is shown after membrane formation and closed system rinsing..

Figure 5.12: POPC:POPS:BP 49.5:49.5:1 membrane patches on PDMS. 105

Figure 5.13: Diffusion coefficients for four different membrane dyes. Colored bars represent the mean for at least 3 measurements, error bars present the standard deviation.

Figure 5.14: Exemplary observations made on a Texas Red and POPGcontaining membrane upon $\mathrm{R}_{6}$ addition $(1 \mu \mathrm{M})$.

Figure 5.15: Results for a POPC:POPG 1:1 membrane with 1\% ATTO488 upon addition of $10 \mu \mathrm{M} \mathrm{R}_{6}$. .

Figure 5.16: Results for in situ POPC:POPG 1:1 membrane staining with Rh18 while $\mathrm{R}_{6}$ was already present $(1 \mu \mathrm{M})$.

Figure 5.17: Fluorescence micrographs of stacks observed on POPC:POPG:BP 49.5:49.5:1 membranes for different peptides.

Figure 5.18: Stack formation on POPC:POPS:BP 49.5:49.5:1 membranes by different peptides.

Figure 5.19: Exemplary RIfS experiment to obtain a dissociation constant for $\mathrm{R}_{4}$ on a POPC:POPG 1:1 membrane.

Figure 5.20: Plots of optical thickness change $\triangle O T$ against R-peptide concentration in $\mu \mathrm{M}$ for POPC:POPG 1:1 membranes, with (left plots) and without (right plots) $1 \%$ Bodipy dye.

Figure 5.21: Plots of optical thickness change $\triangle O T$ against $\mathrm{R}_{6}$ concentration in $\mu \mathrm{M}$ for POPC:POPS 1:1 membranes, with (left plot) and without (right plot) 1\% Bodipy dye.

Figure 5.22: Three models for more highly fluorescent domains on POPG- or POPS-containing membranes in the presence of R-peptides... 120

Figure 5.23: Models for the nature of connections between the lipid stacks and the main bilayer, in general (A-C) and specifically for the homogeneous stacks of the second fluorescence level (D-E). ...122

Figure 5.24: Final hypothesis for the structure of R-peptide induced membrane stacks.

Figure 5.25: Three models for the mechanism of stack formation and the origin of the necessary lipid material. 
Figure 5.26: Hypothesis for dark domains observed with ATTO488-DOPE as a dye;

Figure 6.1: Illustration of arginine-rich CPPs entering cells while they facilitate the formation of stalk-like junctions between bilayer lamellae.

Figure A 1.1: Cumulative plots of normalised experimental data to examine to what extent data distributions can shift without intentionally changing the conditions..

Figure A 1.1: Results of individual breakthrough force spectroscopy experiments on POPC:BP 99:1 membranes with lysine $(K)$. II

Figure A 2.2: Results of individual breakthrough force spectroscopy experiments on POPC:BP 99:1 membranes with $10 \mathrm{mM} \mathrm{N}$ Propylguanidine $\left(R_{\text {Side }}\right)$.

Figure A 2.3: Results of individual breakthrough force spectroscopy experiments on POPC:BP 99:1 membranes with $20 \mathrm{mM} \mathrm{N}$ Propylguanidine $\left(R_{\text {Side }}\right)$.

Figure A 2.4: Results of individual breakthrough force spectroscopy experiments on POPC:BP 99:1 membranes with $10 \mathrm{mM}$ of an arginine dipeptide $\left(R_{2}\right)$.

Figure A 2.5: Results of a breakthrough force experiment on a POPC:BP 99:1 membrane with a hexa-arginine peptide $\left(R_{6}\right)$ in two concentrations. The left plot presents the breakthrough force results, the right plot presents breakthrough distance results. .. VIII

Figure A 3.2.1: Results of individual breakthrough force spectroscopy experiments on POPC:POPG:BP 49.5:49.5:1 membranes with

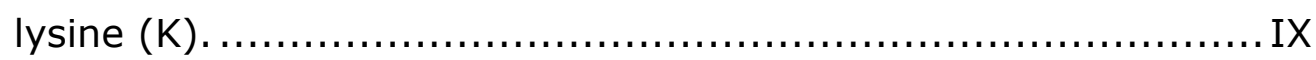

Figure A 3.2: Results of individual breakthrough force spectroscopy experiments on POPC:POPG:BP 49.5:49.5:1 membranes with 10 mM N-Propylguanidine ( Rside $)$. $X$

Figure A 3.3: Results of individual breakthrough force spectroscopy experiments on POPC:POPG:BP 49.5:49.5:1 membranes with 20 mM N-Propylguanidine ( $\left.R_{\text {Side }}\right)$. 
Figure A 3.4: Results of individual breakthrough force spectroscopy experiments on POPC:POPG:BP 49.5:49.5:1 membranes with $10 \mathrm{mM}$ of an arginine dipeptide $\left(\mathrm{R}_{2}\right)$.

Figure A 3.5: Results of a breakthrough force spectroscopy experiment on POPC:POPG:BP 49.5:49.5:1 membrane, with $1 \mu \mathrm{M}$ of a hexaarginine peptide $\left(R_{6}\right)$.

XVI

Figure A 3.6: Results of individual breakthrough force spectroscopy experiments on POPC:POPG:BP 49.5:49.5:1 membranes with $1 \mathrm{mM}$ of a hexa-arginine peptide $\left(\mathrm{R}_{6}\right)$.

XVII

Figure A 4.3.1: Results of individual breakthrough force spectroscopy experiments on POPC:POPE:BP 49.5:49.5:1 membranes with lysine $(\mathrm{K})$

Figure A 4.2: Results of individual breakthrough force spectroscopy experiments on POPC:POPE:BP 49.5:49.5:1 membranes with 10 mM N-Propylguanidine ( Rside $_{\text {) }}$ $\mathrm{XX}$

Figure A 4.3: Results of individual breakthrough force spectroscopy experiments on POPC:POPG:BP 49.5:49.5:1 membranes with 20 mM N-Propylguanidine ( $\left.R_{\text {Side }}\right)$.

Figure A 4.4: Results of individual breakthrough force spectroscopy experiments on POPC:POPE:BP 49.5:49.5:1 membranes with $10 \mathrm{mM}$ of an arginine dipeptide $\left(\mathrm{R}_{2}\right)$.... XXIV

Figure A 4.5: Results of a breakthrough force spectroscopy experiment on POPC:POPE:BP 49.5:49.5:1 membrane, with $1 \mu \mathrm{M}$ of a hexaarginine peptide $\left(R_{6}\right)$. $\mathrm{XXV}$

Figure A 4.6: Results of a breakthrough force spectroscopy experiment on POPC:POPE:BP 49.5:49.5:1 membrane, with $1 \mathrm{mM}$ of a hexaarginine peptide $\left(R_{6}\right)$. XXVI

Figure A 6.5.1: Leakage assay for $R_{6}$ in Hepes buffer with carboxyfluorescein as a dye $\left(\lambda_{e m}=520 \mathrm{~nm}, \lambda_{e x}=490 \mathrm{~nm}\right)$, in POPG-containing vesicles. XXIX

Figure A 6.1: Exemplary data of the diffusion coefficient of BP as measured on the global membrane $(\mathbf{A})$ and the darker areas in the presence of $\mathrm{R}_{\text {Side }}(\mathbf{B})$ $X X X$ 
Figure A 8.1: RIfS experiments with $R_{6}$ addition to POPC:POPS $1: 1$ membranes in the presence of vesicles (due to closed system membrane rinsing).

Figure A 9.1: Bleach profiles (right) of a cross-section through the bleached spot (indicated on left images of a POPC: POPS:BP 49.5:49.5:1 membrane before bleaching, after bleaching and after recovery). XXXII

Figure A 10.1: $Q I^{T M}$ AFM image zoomed in on Figure 5.1 B p. 84 (repeated to the left).. XXXIII

Figure A 11.1 Supplementary fluorescence micrographs. XXXIV

Figure A 11.2: Anecdotal observation of temporary dark domains for POPC:BP 99:1 membranes briefly after addition of $20 \mathrm{mM} \mathrm{R}_{\text {side. }}$. 


\section{List of Tables}

Table 3.1: Buffer compositions used in this work

Table 3.2: Lipid compositions of lipid films used in this work. Dye = Bodipy, Texas Red-DHPE, or ATTO488-DOPE.

Table 5.1: Dissociation constants obtained for investigated lipid mixtures and R-peptides, with and without $1 \%$ Bodipy dye.

Table A 2.1: Means, medians and standard deviations for all breakthrough force spectroscopy experiments on a POPC:BP 99:1 lipid composition, with lysine (K), as shown in Figure A $2.1 \ldots \ldots \ldots \ldots . .$. II

Table A 2.2: Means, medians and standard deviations for all breakthrough force spectroscopy experiments on a POPC:BP 99:1 lipid composition with $10 \mathrm{mM} \mathrm{R}_{\text {side, }}$ as shown in Figure A 2.2......... IV

Table A 2.3: Means, medians and standard deviations for all breakthrough force spectroscopy experiments on a POPC:BP 99:1 lipid composition with $20 \mathrm{mM} \mathrm{R}_{\text {side, }}$ as shown in Figure A 2.3.......... VI

Table A 2.4: Means, medians and standard deviations for all breakthrough force spectroscopy experiments on a POPC:BP 99:1 lipid composition with $10 \mathrm{mM} \mathrm{R}$, as shown in Figure $\mathbf{A} 2.4$.

Table A 2.5: Means, medians and standard deviations of the breakthrough force spectroscopy results presented in Figure A 2.5............VIII

Table A 3.1: Means, medians and standard deviations of datasets presented in the cumulative plots of Figure A $3.2 .1 \ldots \ldots \ldots \ldots \ldots \ldots \ldots$ IX

Table A 3.2: Means, medians and standard deviations of datasets presented

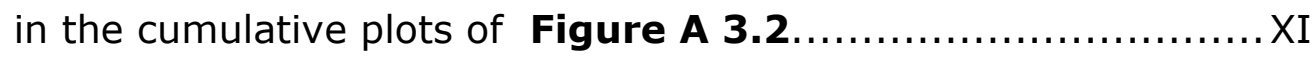

Table A 3.3: Means, medians and standard deviations of datasets presented in the cumulative plots of Figure A 3.3..........................III

Table A 3.4: Means, medians and standard deviations of datasets presented in the cumulative plots of Figure A 3.4. $\mathrm{XV}$

Table A 3.5: Means, medians and standard deviations to the datasets presented in the cumulative plots of Figure A 3.5............... XVI

Table A 3.6: Means, medians and standard deviations to the datasets presented in the cumulative plots of Figure A 3.6. 
Table A 4.1: Means, medians and standard deviations to the datasets presented in the cumulative plots of Figure A 4.3.1............XIX

Table A 4.2: Means, medians and standard deviations to the datasets presented in the cumulative plots of Figure A 4.2 .............XXI

Table A 4.3: Means, medians and standard deviations to the datasets presented in the cumulative plots of Figure A 4.3............XXIII

Table A 4.4: Means, medians and standard deviations to the datasets presented in the cumulative plots of Figure A $4.4 \ldots \ldots \ldots \ldots . . ., X V$

Table A 4.5: Means, medians and standard deviations to the datasets presented in the cumulative plots of Figure A 4.5 .............XXVI

Table A 4.6: Means, medians and standard deviations to the datasets presented in the cumulative plots of Figure A 4.6............ XXVI

Table A 5.1: Elaborate statistical quantities for the normalised breakthrough force results presented in Figure 4.3, Figure 4.5 and Figure 4.6 XXVII

Table A 5.2: Elaborate statistical quantities for the normalised breakthrough force results presented in Figure 4.8. XXVIII 


\section{Abbreviations, physical quantities}

\section{\& symbols}

$\left\langle q^{2}\right\rangle$

[R]

$\Delta U$

$\Delta \varphi$

${ }^{\circ} \mathrm{C}$

$a$

AFM

AMP

ANTS

ATTO488-DOPE

Bodipy

BP

ca.

$\delta$

$\mathrm{CD}$

CLSM

$\mathrm{cm}$

CPP

$D$

DMSO

DMT

DOPC

DOPE

DPX

EDTA

et al.

$E_{\text {tot }}$

ff.

$F(t)$

FIJI

FITC
Mean square spring displacement

R-peptide concentration

Activation energy for rupture of a thin molecular film

Phase difference (in reflected light)

Degrees Celcius

Contact radius of cantilever tip and membrane

Atomic Force microscopy

Antimicrobial peptide

8-Aminonaphthalene-1,3,6-Trisulfonic Acid

ATTO488-DOPE

1,2-Dioleoyl-sn-glycero-3-phosphoethanolamine labelled with ATTO488

$\beta$-BODIPY ${ }^{\text {TM }}$ 500/510 C12-HPC

2-(4,4-Difluoro-5-Methyl-4-Bora-3a,4a-Diaza-s-Indacene-3-Dodecanoyl)-1Hexadecanoyl-sn-Glycero-3-Phosphocholine

$\beta$-BODIPYTM 500/510 C12-HPC

2-(4,4-Difluoro-5-Methyl-4-Bora-3a,4a-Diaza-s-Indacene-3-Dodecanoyl)-1Hexadecanoyl-sn-Glycero-3-Phosphocholine

circa

Indentation depth of a cantilever tip into a deformable sample

Circular dichroism

Confocal laser scanning microscopy

centimetre

Cell-penetrating peptide

Diffusion Coefficient

Dimethylsulphoxide

Dejarguin-Müller-Toporov

1,2-Dioleoyl-sn-glycero-3-phosphocholine

1,2-Dioleoyl-sn-glycero-3-phosphoethanolamine

p-Xylene-Bis-Pyridinium Bromide

Ethylenediaminetetraacetic acid

et alii

Reduced Young's modulus

Foliis, 'and following pages'

Integrated fluorescence intensity

FIJI is just Image]

Fluorescein isothiocyanate 
FRAP

$\Gamma$

GFP

GUV

h

$\mathrm{H}_{2} \mathrm{O}$

$\mathrm{HCl}$

Hepes

HIV

HPLC

$I_{R}$

J

JKR

$\mathrm{K}$

K6

$k_{B}$

$k_{c}$

$\mathrm{KCl}$

$K_{D}$

$\lambda$

LUV

M

$m$

MD

$\mathrm{mg}$

$\mathrm{mL}$

$\mu \mathrm{L}$

$\mu \mathrm{M}$

$\mathrm{mM}$

$\mathrm{NaCl}$

$\mathrm{NaN}_{3}$

$\mathrm{NaOH}$

$\mathrm{nm}$

$\mathrm{nN}$

$\mathrm{N}_{\mathrm{w}}$

OT

p.

pp.

pAntp

PDMS

PEG

PMF
Fluorescence recovery after photobleaching

Line tension

Green fluorescent protein

Giant unilamellar vesicle

Height of a transmembrane cylinder in MD simulations

Molecular film thickness in the molecular model of thin film rupture theory

Water

Hydrochloric acid

4-(2-hydroxyethyl)-1-piperazineethanesulfonic acid

Human immunodeficiency virus

High pressure liquid chromatography

Intensity of reflected light

Joule

Johnson-Kendall-Roberts

Lysine or Kelvin (unit)

Hexalysine, H-Lys-Lys-Lys-Lys-Lys-Lys-OH trifluoroacetate salt

Boltzmann constant $\left(1.38064852 \times 10^{-23} \mathrm{~J} / \mathrm{K}\right)$

Cantilever spring constant

Potassium Chloride

Dissociation constant

Wavelength

Large unilamellar vesicle

Mega or molar

Hill coefficient

Molecular Dynamics

milligram

millilitre

microlitre

micromolar

millimolar

Sodium Chloride

Sodium azide

Sodium Chloride

nanometre

Nano-Newton

Number of water molecules in a transmembrane cylinder in MD simulations Optical Thickness

page

pages

homeodomain of antennapedia gene homeobox of Drosophila Melanogaster

Polydimethylsiloxane

Polyethylene glycol

Potential of mean force 


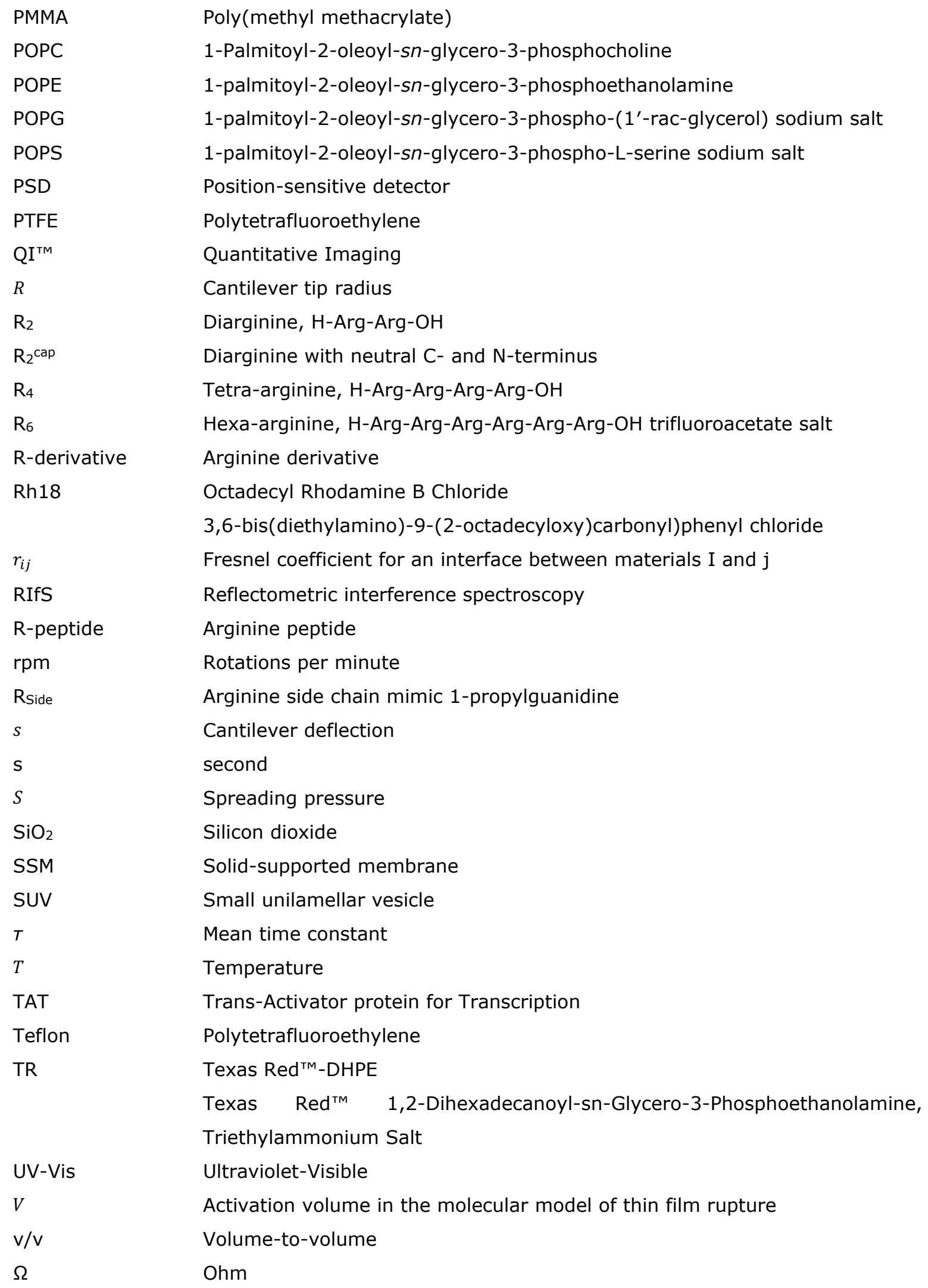

